\title{
Beauty from senselessness: Searching for signals of Beyond the Standard Model physics in a complex world.
}

by

\section{Paul Archer-Smith}

\author{
A thesis submitted to the \\ Faculty of Graduate and Postdoctoral Affairs \\ in partial fulfillment of the requirements \\ for the degree of
}

Doctor of Philosophy in Physics

\author{
Department of Physics \\ Carleton University \\ Ottawa-Carleton Institute of Physics \\ Ottawa, Canada
}

August 25, 2021

Copyright (C) 2021 Paul Archer-Smith 


\section{Abstract}

In this thesis I explore a collection of disparate approaches in the search for new physics beyond the Standard Model (BSM). The research here focuses on the potentially interesting signals that BSM physics could produce; the result is a combination of model building, cosmology, collider physics, and gravitational waves. The signals of the new physics are determined and used to discuss both the discovery potential of next-generation experiments and the parameter space bounds from current collaborations. There are three projects that make up the core of the research presented here.

The first of these explores the possibility of detecting gravitational waves generated by first-order phase transitions in multiple dark sectors. The cosmological history of this framework is outlined and the gravitational wave profiles are generated. These profiles are checked against projections of next-generation gravitational wave experiments, demonstrating that multiple hidden sectors can indeed produce unique gravitational wave signatures that will be probed by these future experiments.

Next up is a study of one of the simplest extensions to the SM: a light Higgs portal scalar. Such a particle could be abundantly produced in the earth's atmosphere and decay in large-volume neutrino detectors. I point out that the Hyper Kamiokande detector bears a strong discovery potential of probing such particles in an uncharted parameter space that is explored by intensity frontier experiments including rare kaon decays. This search can be generalized to other new light states and is highly complementary to beam experiments.

Finally, I explore models of new physics that can give rise to large enhancements to the rate of Higgs decay to $Z \gamma$ while still being consistent with other measurements. I show that this is impossible in simple models with one additional multiplet, and 
also in well motivated models such as the MSSM and folded SUSY. I do find models with several multiplets that carry electroweak charge where such an enhancement is possible, but they require destructive interference effects. I also show that kinematic measurements in Higgs decay to four leptons can be sensitive to such models. Finally, I explore the sensitivity of four lepton measurements to supersymmetric models. 


\section{Acknowledgments}

As with any project that takes several years, the work within this thesis would not have been possible without the massive support I've received over the course of my graduate studies. To all those that helped along the way, thank you: there are many not mentioned here that have helped make my PhD years incredibly enjoyable.

First of all, I must express the deepest of thanks to my supervisor, Dr. Daniel Stolarski. His willingness to put up with all sorts of questions and consistent ability to point me in the right direction were massively important to the development of both my research and my physics understanding. He challenged me in many ways and taught me in even more - I would not be anywhere close to where I am today without his guidance.

Also crucial to my development are the rest of the Carleton theory faculty: Dr. Bruce Campbell, Dr. Stephen Godfrey, Dr. Thomas Grégoire, Dr. Heather Logan, and Dr. Yue Zhang. The final two, in particular, played very important roles in my studies: I learned an incredible amount working on my higgs portal project with Yue Zhang and the QFT taught by Heather Logan was instrumental in building up my research. In addition, I would like to thank Dr. Tom Steele for his excellent instruction and supervision during my masters degree.

There are two other graduate students who taught me so much about physics over the years that I am forever indebted to them: Dr. Kevin Earl and Dr. Fred Sage. Both helped teach me how to really get into the calculations of QFT, and without their help I have no idea where I would be today. On the research side, Dylan Linthorne was excellent both as a co-author and as an office mate - I very much appreciate the work that we've done together over the last several years. Also deserving of recognition are the other grad students with whom I had awesome discussions: Jérôme Claude, Dr. 
Jason Ho, Taylor Gray, Roozbeh Yazdi, Hassan Essa, and Ben Keeshan.

Over the past year and a half, during the pandemic and various lockdowns, I must thank Sophie LeClair-Roberson, Lindy Van Vliet, and Narges Zare for keeping me sane and on track during crazy times.

I would like to thank my parents, Jan and Lyndon, and my siblings, Joel and Lindsay, for putting up with my shenanigans for years.

Last and certainly not least, I want to thank my girlfriend Kaitlin for supporting me throughout the entire journey; her love and care are some of the brightest spots each and every day. 


\section{Statement of Originality}

The background chapters $2,3, \& 4$ are reviews of the SM, cosmology, and relevant BSM models, respectively. The material for the first two is well established and has entered the realm of textbooks (with the exception of Sec. 3.5.1, which contains original material from [1]); whereas the BSM chapter contains work that is more modern and somewhat speculative. In all cases, sources are listed at the beginning of the relevant chapter.

The following chapters 5, 6, \& 7 contain original research. Each of these projects were done in collaboration with other physicists with contributions as follows:

\section{Gravitational Wave Signals from Multiple Hidden Sectors [1]:}

This project was done in collaboration with Dylan Linthorne and Daniel Stolarski. The particle physics calculations were carried out by P.A.S including the reheaton decays, $N_{\text {eff }}$ constraints, phase transition temperatures, hidden sector energy densities, and thermal evolution of the hidden sectors. These results were utilized by Dylan Linthorne to calculate and plot the gravitational wave profiles against the next-generation detector limits. The detector limits and gravitational wave profiles were checked by P.A.S.

\section{Higgs Portal from the Atmosphere to Hyper-K [2]:}

This work was done in conjunction with Yue Zhang. The original geometry work was completed by Yue Zhang. P.A.S checked these results and then applied them in a semi-analytical calculation determining the rate and kinematics of scalar portal particle decays in large-volume neutrino detectors. The energy and kinematic distributions were plotted by P.A.S. The number of signal events 
over ten years were calculated by P.A.S; these results were used by Yue Zhang to generate the contour plots of Fig. 6.3.

3. On New Physics contribtuions to the Higgs decay to $Z_{\gamma}[3]$ :

The final project was produced in collaboration with Daniel Stolarski and Roberto Vega-Morales. All calculations for this project were carried out by P.A.S. Code written by Roberto Vega-Morales was utilized in the 4-lepton analysis portion to determine the number of signal events required for statistical discrimination of SM and BSM scenarios. 


\section{Contents}

1 Preface 1

2 The Standard Model 5

2.1 Introduction . . . . . . . . . . . . . . . . . . 5

2.2 Quantum field theory . . . . . . . . . . . . . . 6

2.2.1 Motivations and history . . . . . . . . . . . 6

2.2.2 Classical Fields . . . . . . . . . . . . . . . . . . . . . 7

2.3 The Lorentz group and discrete symmetries . . . . . . . . . . . . 10

2.4 The Lagrangians of quantum fields . . . . . . . . . . . . . . 15

2.5 Non-Abelian gauge theories . . . . . . . . . . . . . . . 22

2.6 Field content of the Standard Model . . . . . . . . . . . . . . . . . . 27

2.7 The Higgs and electroweak symmetry breaking . . . . . . . . . . . . 33

2.8 Challenges to the $\mathrm{SM} \ldots \ldots \ldots$. . . . . . . . . . . . 40

3 Cosmology 43

3.1 Introduction . . . . . . . . . . . . . . . . . . 43

3.2 History of the Universe . . . . . . . . . . . . . . . . . . . 44

3.3 Dynamics of the Universe . . . . . . . . . . . . . . . . . . . . 48

3.4 Particles in the standard cosmology . . . . . . . . . . . . . . 53 
3.5 Gravitational waves . . . . . . . . . . . . . . . . 58

3.5.1 Detection of Stochastic Graviational Waves . . . . . . . . . . 62

4 Beyond the Standard Model $\quad 66$

4.1 Hidden sector portals . . . . . . . . . . . . . . 67

$4.2 \quad$ Nnaturalness . . . . . . . . . . . . . . . . 70

4.3 Supersymmetry . . . . . . . . . . . . . . . . . . . . 75

4.3 .1 Overview . . . . . . . . . . . . . . . 75

$4.3 .2 \quad$ Stop Sector . . . . . . . . . . . . . . . . . 80

5 Gravitational Wave Signals from Multiple Hidden Sectors 85

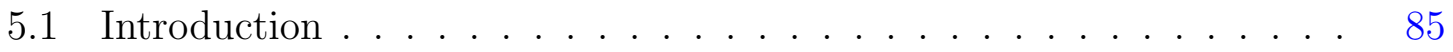

$5.2 \quad$ Particle Setup . . . . . . . . . . . . . . . . . . 88

5.3 QCD Phase Transition . . . . . . . . . . . . . . . 90

$5.3 .1 \quad$ Standard Sectors . . . . . . . . . . . . . . . . . . 92

$5.3 .2 \quad$ Exotic Sectors . . . . . . . . . . . . . . . . . . . . . . 93

5.4 Reheating $N$ Sectors $\ldots \ldots \ldots \ldots \ldots \ldots \ldots$

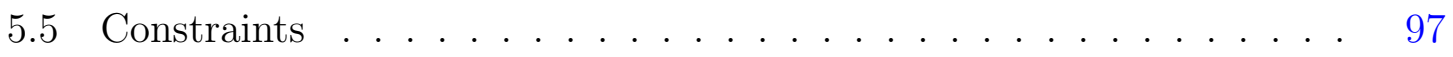

5.5.1 Exotic Sector Contributions . . . . . . . . . . . . . . . 99

5.5.2 Standard Sector Contributions . . . . . . . . . . . . . 100

5.5.3 Generalized Reheating Scenarios . . . . . . . . . . . . . . 101

5.6 Gravitational Wave Signals . . . . . . . . . . . . . . . . . . . . . . 102

5.6.1 Production of Gravitational Waves _. . . . . . . . . 105

5.6.2 Detection of Stochastic Graviational Waves . . . . . . . . . . 114

5.7 Nonrunaway phase transitions . . . . . . . . . . . . . 116

5.8 Conclusion . . . . . . . . . . . . . . . . . . . . . . . . . . . . . . . 119 
7 On New Physics Contributions to the Higgs Decay to $Z_{\gamma} \quad 137$

7.1 Introduction . . . . . . . . . . . . . . . . . . . 137

7.2 Higgs Physics . . . . . . . . . . . . . . . . . . . . . 142

$7.2 .1 h \rightarrow 4 \ell$ at One Loop . . . . . . . . . . . . . . . . . 143

7.2 .2 Kinematic Analysis of $h \rightarrow 4 \ell \ldots \ldots \ldots \ldots$

7.3 Models with large $h \rightarrow Z \gamma$ contributions $\ldots \ldots \ldots \ldots \ldots$

7.3.1 New Scalar Multiplets . . . . . . . . . . . . . . 150

7.3.2 The Failure Of One Additional Multiplet . . . . . . . . . . . 152

7.3.3 The Singlet-Triplet Model . . . . . . . . . . . . . . . . . 153

7.3.4 Four-Lepton Sensitivity . . . . . . . . . . . . . . . . 157

7.4 Supersymmetric Models . . . . . . . . . . . . . . . . . . . . . 159

7.4.1 Four-Lepton Analysis . . . . . . . . . . . . . . . . . . 162

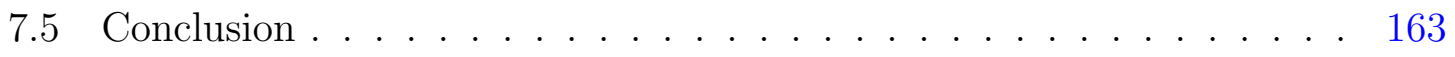

8 Summary of Results 168

9 Epilogue 171

$\begin{array}{ll}\text { A Conventions } & 173\end{array}$

$\begin{array}{ll}\text { B Loop Integrals } & 175\end{array}$ 


\section{List of Tables}

2.1 Symmetries of the Standard Model. . . . . . . . . . . . . . . . . 27

2.2 Field content of the Standard Model. The flavour index $m$ goes from 1 to 3 and indicates the generation of the fermions. $a$ from 1 to 3 and $b$ from 1 to 8, indicating the gauge bosons for $S U(2)$ and $S U(3)$, respectively. The $L$ and $R$ subscripts for the fermion multiplets represent the multiplet's chirality. The numbers in the $S U(3)_{C}$ and $S U(2)_{L}$ columns show the representation of the various multiplets under the respective symmetry; the $U(1)_{Y}$ column denotes the hypercharge of the multiplets. 30

3.1 History of the universe. . . . . . . . . . . . . . . . . . 45

3.2 Key properties of particles in thermal equilibrium. $\zeta$ is the Riemann zeta function. ........................ 55

4.1 Types of portals and their respective lowest-order couplings. $F_{\mu \nu}^{\prime}, B_{\mu \nu}, G_{\mu \nu}, F_{\mu \nu}$ are the dark photon, hypercharge, gluon, and photon field strength tensors respectively. $S$ represents a new scalar singlet, $a$ a pseudoscalar axion, and $N$ a new neutral fermion. . . . . . . . . . . 
4.2 Supermultiplets of the MSSM and their particle content. There are 3 generations of the quark and lepton supermultiplets. The first 7 entries are chiral supermultiplets, while the final three are gauge supermultiplets. The final column gives the supermultiplet representation under $S U(3)_{C} \times S U(2)_{L} \times U(1)_{Y} \ldots \ldots \ldots 78$

5.1 Outline of parameters used for the various multi-hidden-sector scenarios. The Higgs VEV is the VEV for the given additional sector, $\Lambda_{Q C D}^{A S}$ is the QCD phase transition in the additional sector, and $T_{\gamma}$ is the temperature of the SM photon bath when the SFOPT occurs in the additional sector. The index indicates the equivalent sector from the Nnaturalness model (Eq. (5.4)). It should be noted that although the various sectors undergo phase transitions at different temperatures, they are all assumed to be reheated to the same initial temperature. . 112 


\section{List of Figures}

3.1 The evolution of effective relativistic degrees of freedom $\left(g_{\star \epsilon}\right)$ as a function of temperature. Also included are effective degrees of freedom for number density $\left(g_{\star n}\right)$, pressure $\left(g_{\star p}\right)$, and entropy $\left(g_{\star s}\right)$. From [32]. . 56

3.2 Schematic of a Michelson gravitational wave interferometer. The beam from the laser is divided by a beam splitter such that two separate beams travel along the two paths to the mirrors before being reflected back towards the beam splitter and being recombined and detected. [42] 63

4.1 Sketch of the Nnaturalness set-up. [57] . . . . . . . . . . . . . . 71

4.2 Decays for a scalar reheaton. The left (right) column is for the standard (exotic) sectors while the top (bottom) row is for $m_{\phi} \gg\left|m_{H}\right|\left(m_{\phi} \ll\right.$

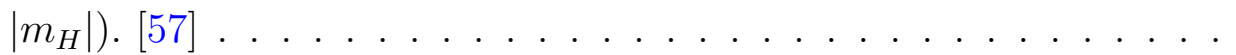

4.3 Energy densities as a function of sector index normalized to the energy of the SM sector for a $100 \mathrm{GeV}$ reheaton. The blue line represents the scaling for standard sectors while the purple line shows the energy density for exotic sectors. The drops in the standard sector energy density correspond to thresholds of the form $m_{\phi} \ll 2 m_{c_{i}}$ where a quark species becomes too heavy for the reheaton to decay to. $[57] \ldots$. . 
4.4 Contribution of the top and its partners to the Higgs mass. The quadratic divergences cancel. $[60] \ldots \ldots$. . . . . . . . . . .

5.1 Gravitational wave spectral energy density (solid curves) for standard Nnaturalness using the scalar reheaton model of section 5.4. The curve corresponding to the sum of the sectors is approximately equal to the $i=1$ curve. All contributions are assumed to be purely from bubble collisions $\Omega_{\phi}$. The colored solid lines use $\beta / H=10$ whereas the dashed gray line is the total contribution of all sectors for $\beta / H=10^{4}$ (the sum of all sectors is roughly equal to the $i=1$ curve and sectors beyond the first are below the range of this plot). The shaded dashed curves are the power law noise curves [47] calculated from expected sensitivity as described in Section 5.6.2. The ones on the right are space-based interferometers: LISA [44] (blue), DECIGO [17] (light blue), BBO [45] (red). The ones on the left are for the pulsar timing array SKA [52] for exposure time of 5-years (purple), 10-years (orange), and 20-years

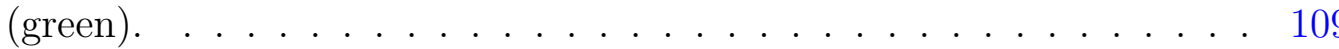

5.2 Gravitational wave spectral energy density for the various scenarios found in Table 5.1. All contributions are assumed to be purely from runaway bubble collisions $\Omega_{\phi}$. The colored solid lines use $\beta / H=10$ where as the dashed gray line is the total contribution of all sectors for $\beta / H=10^{4}$. The inset is a closer look at the region around the peaks for the $\beta / H=10$ case. The shaded curves are the same as Fig. 5.1. . 114 
5.3 Gravitational wave spectral energy density for the various scenarios found in Table 5.1. All contributions are assumed to be from a nonrunaway phase transition with terminal velocity $v=0.95$. The coloured solid lines use $\beta / H=10$ whereas the dashed grey line is the total contribution of all sectors for $\beta / H=10^{4}$. The top left figure shows the individual sound wave and MHD contributions. The shaded curves are the same as Fig. 5.1. In contrast to the runaway case, most scenarios evade the projected sensitivities. . . . . . . . . . . . . .

6.1 Energy distribution of atmospheric kaons $\left(K^{ \pm}\right.$and $K_{L}$ added together) and $\phi$ particles, for $m_{\phi}=150 \mathrm{MeV}$, obtained from the atmospheric simulation described in the text. For illustration purpose, the flux of $\phi$ has been rescaled by assuming the $K \rightarrow \pi \phi$ decay branching ratios are equal to $1 . \ldots \ldots \ldots \ldots \ldots \ldots \ldots$

6.2 Geography of earth and detector. The blue box indicates the location of the Hyper-K detector. The dashed circle represents a sphere where the cosmic-ray-atmosphere reactions mainly occur that produce light $\phi$ particles. $h$ is given by the height of this sphere plus the depth of detector underground, and $\varphi$ is the zenith angle in view of the detector. 127

6.3 Caption next page. . . . . . . . . . . . . . . . 130 
6.3 (Previous page.) Upper: Using Hyper-K detector to search for long lived Higgs portal scalar $\phi$ produced from the atmosphere. The (solid, dashed, dotted) black contours correspond to 10, 100, 1000 signal events after ten years of exposure. The red curves correspond to constant values of $c \tau_{\phi}$, the lifetime of $\phi$ times the speed of light. Middle: Hyper-K region (the three gray contours in the background are the same as those black ones in the upper panel) shown together with the existing constraints (from E949, NA62, CHARM, LSND, LHCb, Belle) and future reach by the upcoming experiments (ICARUS, SBND). Lower: The region of parameter space favored by the earlier KOTO excess is shown by the blue bands (dark and light blue correspond to 1 and $2 \sigma$ favored regions, respectively) [138-140]. The upper bound on the mixing angle $\theta$ derived from the latest KOTO analysis [141] is shown by the thick dark blue curve. The union of existing constraints excludes the gray shaded region. Like the upper and middle panels, the black curves corresponds to fixed number of signal events using Hyper-K to hunt atmospheric $\phi$ particles. For comparison, we also show the rescaled 10, 100, 1000 signal event contours (in pink color) for Super-K with 328 kiloton-year of data. In all the plots, the blue star corresponds to the benchmark point used in Eq. (6.11) and Fig. 6.4.131 
6.4 Additional kinematical features of the $\phi$ decay signal. Top: Zenith angle $\varphi$ distribution of the incoming into the Hyper-K detector for two sets of parameters, $m_{\phi}=150 \mathrm{MeV}, \theta=5 \times 10^{-4}$ (red) and $m_{\phi}=150 \mathrm{MeV}, \theta=10^{-4}$ (blue). The first point corresponds to the blue star in Fig. 6.3 and can leads to hundreds of $\phi$ decay events in Hyper-K. Most of the events are expected to arrive in directions above the detector's horizon. Bottom: electron-positron opening angle distribution from $\phi$ decay, for $m_{\phi}=150 \mathrm{MeV}$. The corresponding $\phi$ energy spectrum is shown in Fig. 6.1. . . . . . . . . . . . . . . . . 134

7.1 NP scalar one-loop contributions to Higgs to neutral diboson decays. The new scalar is represented by the unlabeled dashed lines. . . . . . 139

7.2 Representation of the $h V V$ corrections to the $h \rightarrow 4 l$ amplitude where $V_{1,2}=Z, \gamma$ and $\ell, \ell^{\prime}=e, \mu . \ldots \ldots \ldots \ldots \ldots \ldots$

7.3 Likelihood contours in the $\kappa_{\gamma} \kappa_{g}$ plane from ATLAS data [178]. All other coupling modifiers are fixed to the SM values. . . . . . . . . . 144

7.4 Number of Higgs to four lepton events $N$ required to distinguish the SM from a large $Z \gamma$ model benchmark at a given significance $\sigma$. The top figure shows the curve fitted to the average statistical significance of pseudo-experiments featuring a given number of events (the red points). The bottom figure shows an extrapolation of this data with lines at 2 and $3 \sigma$ significance. The blue shaded region shows the expected reach of the HL LHC. . . . . . . . . . . . . . . . . 158 
7.5 Exclusion bounds in stop parameter space due to $h \rightarrow \gamma \gamma$ and gluon fusion measurements: the solid rust (black) lines represent the 2 (1) sigma exclusion bounds for stops and the dashed green (yellow) lines represent the 2 (1) sigma exclusion bounds for F-stops. The top figure is in the stop mass 1, stop mass 2 plane with zero mixing. The bottom figure is in the stop mass 1, stop mixing angle plane, with stop mass 2 held at $1 \mathrm{TeV}$. The region to the left of the curves is excluded. . . . 166

7.6 Number of Higgs to four lepton events $N$ required to distinguish the SM from the SUSY model benchmarks at a given significance $\sigma$. The top figure shows the curve fitted to the average statistical significance of pseudo-experiments featuring a given number of events. The dashed violet line (fit to the green carets) is the stop scenario and the solid black line (fit to the red asterisks) is the F-stop scenario. The bottom figure shows an extrapolation of this data with lines at 2 and $3 \sigma$ significance. The thin blue band on the left is the reach of the HL-LHC. 167 


\section{Chapter 1}

\section{Preface}

There's a common trope in fantasy: a small, weak, and otherwise insignificant character ends up playing the pivotal role in the clash between good and evil - ultimately placing the grandest of historical events in the hands of the meek. We think of stories about David, Joan of Arc, and hobbits, each playing a role on a scale so much larger than themselves such that any initial estimation is left utterly useless. Such underestimation is not, however, limited to history or mythology: in a strange twist of life imitating art, the universe reprises this narrative with the world on the smallest of scales ultimately dictating the existence and evolution of stars, galaxies, and space itself.

Of course, in a post metanarrative world, the simple black and white dichotomy breaks down, and the interpretation of both literature and history becomes less an understanding of a singular narrative arc and more a reflection of the lens used for viewing it. In this light too, physics reflects philosophy: the physical pictures we

utilize are effective theories valid for certain energy scales. Take Newton's laws [4], for example: nothing about classical mechanics is wrong, per sé, but the classical perspective only functions at speeds well below the speed of light and at sizes far 
larger than an atom. Beyond these limits, things break down and a new theory is required.

These two concepts bring us to the current state of particle physics, a superposition of mystery and certainty. The certainty is quite well founded; the currently accepted Standard Model (SM) of particle physics was dreamed up decades ago and has repeatedly proven its worth against multitudes of data. All the particles predicted by the SM have been discovered at colliders, with the final piece of the puzzle, the higgs boson, being confirmed by the ATLAS and CMS collaborations in $2012[5,6]$. In the near decade since, no new particles have been discovered and precision tests of the SM have returned no signs of new physics $(\mathrm{NP})^{1}$. So, is particle physics complete?

Absolutely not.

As rock solid as the SM has proven itself to be, there is an overwhelming amount of evidence that it is only a fraction of a much larger picture. The most obvious limitation is that the first force accurately described by Newton ${ }^{2}$, gravity, is completely absent from the theory. So too is the dark matter that is thought to make up roughly $85 \%$ of the total matter in the universe [7]. Further, the neutrinos predicted by the SM are massless, however the discovery of neutrino oscillations by Super Kamiokande and the Sudbury Neutrino Observatories indicates that this isn't the case $[8,9]$.

On a theoretical level, even more issues come to the fore. Most notable, perhaps, is the hierarchy problem [10] — why is there such a large separation of scales between gravity and the electroweak force? More explicitly, why does the higgs boson mass not suffer huge quantum corrections on order of the Planck mass? If it does, why is its mass of $125 \mathrm{GeV}$ so much smaller than $\Lambda_{P l} \sim 10^{19} \mathrm{GeV}$ ?

\footnotetext{
${ }^{1}$ Results like the flavour anomalies do exist, but have not reached the level of discovery at the time of writing.

${ }^{2}$ In the classical regime, of course!
} 
Since leaving such questions unaddressed is nigh impossible for our tribe of nearly hairless apes, the quest for developing theories that go Beyond the Standard Model (BSM) is one of the main drivers of 21st century particle physics. Over the past 40 years hundreds of excellent ideas (and thousands of terrible ones) have been presented, but no BSM model has yet emerged triumphant. It is in front of this backdrop that the research presented in this thesis takes place; an attempt to push forward our understanding of BSM models and their signals. There are three projects that make up the core of this work. Each of these papers focuses on different types of both models and signals. Cynically, this might appear to be due to a lack of faith in any particular BSM framework; unfortunately, this is completely correct. In all seriousness, we truly do not know where NP is hiding and what form it takes - as such, it is best to explore as many avenues as possible to see if anything exciting turns up.

The first project, which is based on [1], examines the potential of detecting stochastic gravitational wave backgrounds generated by first-order phase transitions in multiple dark sectors at next-generation gravitational wave experiments. $N$ naturalness is taken as a sample model as it contains many additional sectors that undergo appropriate phase transitions. This framework is then generalized to examine a range of multiple hidden sector scenarios and the gravitational wave backgrounds produced by their phase transitions. The gravitational wave profiles for these scenarios are produced and checked against future projections for pulsar timing array and space-based interferometer experiments; we ultimately show that some of these scenarios do indeed produce unique gravitational wave signatures that can be detected by these experiments.

The second paper [2] studies a simple extension to the SM in the form of a light higgs portal scalar. Particles of this type can be produced in large numbers via 
interactions of cosmic rays with the Earth's atmosphere and, should they be long-lived enough, can traverse the rest of the atmosphere (and possibly through the Earth) and decay within large-volume neutrino detectors. In particular, we demonstrate that Hyper Kamiokande will be able to probe parts of the uncharted scalar portal parameter space. The key signal to look for is electron-positron pair creation that appears as a double-ring, typically originating from zenith angles above the detector's horizon.

The third and final work [3] entertains the concept of BSM models that give rise to large (100\% or more) enhancements to the higgs decay $h \rightarrow Z \gamma$ while not breaking limits placed by other measurements (most notably $h \rightarrow \gamma \gamma$ ). We demonstrate that such large modifications are impossible in both simple models with one additional multiplet as well as more well-established models like the MSSM or folded SUSY. It is shown that large modifications to the $Z \gamma$ decay rate are possible in models that feature several multiplets that carry electroweak charge, but assiduous cancellations through destructive interference effects must take place. 4-lepton analysis of these models is undertaken and reveals that although kinematic measurements of $h \rightarrow$ $4 l$ can be sensitive to large $Z \gamma$ models at the high luminosity LHC, sensitivity to supersymmetric models requires a future high energy collider.

The rest of the thesis is organized as follows. Chapters $2,3, \& 4$ provide background information about the Standard Model, Cosmology, and relevant BSM models. The gravitational wave phenomenology of multiple hidden-sector models is covered in Chapter 5. This is followed by a study of scalar higgs portal models and their potential signals in large-scale neutrino detectors in Chapter 6. The final research work on BSM models featuring large $h \rightarrow Z \gamma$ decays and their collider phenomenology makes up Chapter 7. Results of the three research chapters are summarized in Chapter 8 and everything is wrapped up in Chapter 9. 


\section{Chapter 2}

\section{The Standard Model}

\section{$2.1 \quad$ Introduction}

At the core of essentially all current particle physics research is a theoretical model so absolutely dominant and so incredibly successful in its predictions that it has come to be referred to as The Standard Model (SM). Building on the concepts of gauge theory, the unification of electromagnetism and the weak force, asymptotic freedom, the Higgs mechanism, and more, the SM was formalized in the early-to-mid 70s [11-13]: a gauge quantum field theory (QFT) that describes the electromagnetic, weak, and strong forces. Mathematically, the SM features a gauge group of $S U(3)_{C} \times S U(2)_{L} \times U(1)_{Y}$ - where the first group describes the internal symmetries for colour charge, the second for weak isospin, and the third for weak hypercharge.

Over the decades following its formulation, the SM has held up again and again and again, with all experimental results showing its accuracy to ever greater degrees of precision. As mentioned in Chapter 1, the SM accounts for all known particles. However, there are issues that the SM does not explain: gravity is completely ignored and dark matter is unaccounted for, just to name two serious shortcomings. Exploring 
models that go Beyond the Standard Model (BSM) is the central focus of this thesis and is explored in Chapter 4 onwards. Yet before we venture into those exciting waters, we need to build up the foundational ideas of the SM that form the starting point for all the escapades into the wilderness. So, for now, let us toss aside all the talk of incompleteness, all the unproven (but exciting!) new theories, and all the mysteries of the unknown in favour of bedrock.

In this chapter, we build up the framework of the SM in a rather typical way: QFT and its core ideas are introduced, followed by a discussion of gauge theories and the symmetries of the SM Lagrangian, then electroweak symmetry breaking, and finally the concept of hierarchy. Everything within this chapter is well-known physics, with the main sources being: Peskin and Schroeder's "An Introduction to Quantum Field Theory" [14], Matthew Schwartz's "Quantum Field Theory and the Standard Model" [15], and Heather Logan's TASI 2013 lectures [16].

\subsection{Quantum field theory}

\subsubsection{Motivations and history}

The development of classical electromagnetism in the mid-19th century, special relativity in 1905, and finally quantum mechanics through the 1920s led to an understanding of many microscopic phenomena including atomic spectra, blackbody radiation, and the photoelectric effect. However, crucially, quantum mechanics could not describe the creation and annihilation of particles, nor could it explain spontaneous emission. These deficiencies, combined with a desire to unify the results from the above disparate theories, resulted in the likes of Dirac, Heisenberg, Jordan, and many more laying the foundations of the first QFT, Quantum Electrodynamics (QED). This was done via the development of canonical quantization and the deriv- 
ing of wave equations for relativistic particles — most notably the Dirac equation for electrons.

These early results were able to explain the spin and magnetic moment of the electron, predicted antimatter, and allowed for Fermi to develop a theory of beta decay. Yet in the midst of these accomplishments, a problem loomed. The weapon of choice for physicists studying anything beyond the most basic of systems perturbation theory — had a problem: higher-order perturbative calculations resulted in infinite quantities. It would take nearly two decades before this problem was solved by Tomonaga, Schwinger, and Feynman through the introduction of a procedure known as renormalization [17-19]. Renormalization permitted the calculation of QFT perturbative series to arbitrary order and completed the QFT framework.

Ultimately, QFT as it exists today was successful in completing the fusion of classical field theory, quantum mechanics, and relativity. Although much (perhaps all) of QFT is relevant to both the SM and BSM calculations completed within this thesis, the topic is far too vast to cover here. As such, I focus on symmetry and how it leads to an understanding both of spacetime and the structure of the SM and beyond.

\subsubsection{Classical Fields}

The concept of the Lagrangian originated in the classical sphere of physics in the late $18^{\text {th }}$ century when Joseph-Louis Lagrange developed a reformulation of Newtonian mechanics [20]. This powerful reformulation allowed for the easy induction of generalized coordinates into classical problems - making it far simpler to take constraints into consideration. The crux of this reconstruction of mechanics lies in the Lagrangian, defined as:

$$
L=T-V
$$


Additionally, from Lagrangian field theory the so-called Lagrangian density is written as:

$$
L=\int \mathcal{L} \mathrm{d}^{3} x
$$

with the action $S$ of a system being determined by integration of the Lagrangian

$$
S=\int L \mathrm{~d} t .
$$

Now, in order to express this concept in a form more conducive to the fields used in QFT the Lagrangian density is substituted into (2.3):

$$
S=\int \mathcal{L}\left(\phi, \partial_{\mu} \phi\right) \mathrm{d}^{4} x .
$$

where $\phi$ is a scalar particle field.

Two important distinctions must be made at this point: firstly, the term "the Lagrangian" will be used in place of "the Lagrangian density" for brevity, and, secondly, it will be assumed that $\mathcal{L}$ is dependent only on $\phi$ and its first derivatives for three primary reasons [15]: 1) this simplifies calculations by requiring only the initial positions and velocities to specify boundary conditions, 2) this reflects the form of classical Lagrangians, and 3) this avoids potentially disastrous consequences such a vacuum decay. Higher derivative terms are generically present in all Effective Field Theories (EFT), but these complications can be ignored for our current purposes.

The most significant result arising from the Lagrangian formulation grows out of applying variational parameter methods to the action of a system - generating the Euler-Lagrange equation of motion for the field $\phi$,

$$
\frac{\partial \mathcal{L}}{\partial \phi}-\frac{\partial}{\partial x^{\mu}}\left[\frac{\partial \mathcal{L}}{\partial\left(\partial_{\mu} \phi\right)}\right]=0 .
$$


Beyond the construction of a host of different Equations of Motion (EOM), variational techniques can be applied to further explore the nature of the Lagrangian formulation of relativistic mechanics. The most crucial result is Noether's theorem: a fundamental proof that demonstrates physical symmetries lead to conserved currents and corresponding conservation laws. More explicitly, if a Lagrangian features a continuous symmetry then there exists a current associated with that symmetry which is conserved when the equations of motion are satisfied.

We begin exploring Noether's theorem by deforming the field $\phi$ in the following manner:

$$
\phi \Rightarrow \phi^{\prime}=\phi+\alpha \Delta \phi .
$$

If this transformation is a symmetry then it should leave the equations of motion unchanged. Since we can allow the action to change by a surface term without affecting the equations of motion, the Lagrangian must be invariant under Eq. 2.6 up to a 4-divergence

$$
\mathcal{L} \Rightarrow \mathcal{L}^{\prime}=\mathcal{L}+\alpha \partial_{\mu} J^{\mu}
$$

Variation of the fields leads to the final result:

$$
\partial_{\mu} j^{\mu}(x)=0 \text { and } j^{\mu}(x)=\left[\frac{\partial \mathcal{L}}{\partial\left(\partial_{\mu} \phi\right)}\right] \delta \phi-J^{\mu} .
$$

This result indicates that the current $j^{\mu}(x)$ is conserved as, the charge $Q$,

$$
Q \equiv \int d^{3} x j_{0}
$$

is constant in time

$$
\partial_{t} Q=\int d^{3} x \partial_{t} j_{0}=\int d^{3} x \nabla \cdot \vec{j}=0
$$


where the final step assumes that $\vec{j}$ vanishes on the spatial boundary (in other words, we have assumed that nothing is leaving the boundaries of the system we're examining).

It should be noted that Noether's theorem holds for global symmetries: gauge symmetries (which are local) imply global symmetries and thus conserved currents. In a broader sense, this result accounts for the conservation laws we see in physics: energy, momentum, angular momentum, and quantum numbers (like electric charge) all arise due to the symmetries present in the Lagrangian.

\subsection{The Lorentz group and discrete symmetries}

As QFT is the unification of special relativity and quantum mechanics, it should come as no surprise that Lorentz invariance is a fundamental symmetry of the theory. A generalization of rotational invariance, Lorentz invariance asserts that the laws of physics must remain unchanged under both boosts and rotations. Lorentz transformations can be combined with translations to obtain the full Poincaré group thus describing the full symmetry of special relativity.

Using the standard conventions for particle physics (outlined in Appendix A), Lorentz transformations acting on 4 -vectors are defined as matrices $\Lambda$ that obey

$$
\Lambda^{T} g \Lambda=g=\left(\begin{array}{cccc}
1 & 0 & 0 & 0 \\
0 & -1 & 0 & 0 \\
0 & 0 & -1 & 0 \\
0 & 0 & 0 & -1
\end{array}\right) \text {. }
$$

where $g_{\mu \nu}$ is the typical flat-space Minkowski metric. Lorentz transformations con- 
serve inner products in Minkowski space

$$
V_{\mu} W^{\mu}=V_{0} W^{0}-V_{1} W^{1}-V_{2} W^{2}-V_{3} W^{3}
$$

Four-dimensional matrix representations of these transformations clearly show the similarities to the rotations group in the following examples

$$
\left(\begin{array}{cccc}
1 & 0 & 0 & 0 \\
0 & 1 & 0 & 0 \\
0 & 0 & \cos (\theta) & \sin (\theta) \\
0 & 0 & -\sin (\theta) & \cos (\theta)
\end{array}\right) \quad\left(\begin{array}{cccc}
\cosh (\beta) & \sinh (\beta) & 0 & 0 \\
\sinh (\beta) & \cosh (\beta) & 0 & 0 \\
0 & 0 & 1 & 0 \\
0 & 0 & 0 & 1
\end{array}\right)
$$

where the first matrix represents a rotation of angle $\theta$ about the $x$-axis and the second a boost of rapidity $\beta$ in the $x$ direction.

Looking at Eq. 2.12, it becomes apparent that two discrete symmetries also fit into our definition of Lorentz transformations. Parity, which flips the spatial components of a vector

$$
P:(t, x, y, z) \rightarrow(t,-x,-y,-z)
$$

and time reversal, which — surprise, surprise — flips the time component

$$
T:(t, x, y, z) \rightarrow(-t, x, y, z)
$$

round out the allowed Lorentz transformations according to Eqns. 2.11 \& 2.12.

Under the aforementioned Lorentz transformations, scalars (including scalar fields) are Lorentz invariant $\phi \rightarrow \phi, 4$-vectors are Lorentz covariant and transform 
as a vector field $V^{\mu} \rightarrow \Lambda_{\nu}^{\mu} V^{\nu}$, and tensors transform as $T^{\mu \nu} \rightarrow \Lambda_{\alpha}^{\mu} \Lambda_{\beta}^{\nu} T^{\alpha \beta}$ (of course, higher order tensors are also possible, with the number of indices dependent on the rank). As we ultimately embed particles into fields, these transformation properties are of paramount importance.

On a more mathematical level, the generators of the proper orthochronous Lorentz group (which only include transformations that are continuously connected to the identity) can be written as

$$
\Lambda=\exp \left(i \theta_{i} J_{i}+i \beta_{i} K_{i}\right)
$$

where $J_{i}$ generates rotations and $K_{i}$ generate boosts. Since the Lorentz group preserves a metric, it must be an orthogonal group: $O(1,3)$.

Understanding how the multiplication rules work for the generators of this group is of vital importance: to do this we look to the Lie algebra of the generators of the Lorentz group. Formally, within a Lie algebra the multiplication rules are defined through Lie brackets. When dealing with matrix representations, a Lie bracket is simply the familiar commutator. For the Lorentz algebra, so $(1,3)$, we have the defining relationships:

$$
\left[J_{i}, J_{j}\right]=i \epsilon_{i j k} J_{k}, \quad\left[J_{i}, K_{j}\right]=i \epsilon_{i j k} K_{k}, \quad\left[K_{i}, K_{j}\right]=-i \epsilon_{i j k} J_{k}
$$

It should be noted that this definition for the Lie algebra of the Lorentz group holds 
for any representation and we can combine the generators into a $4 \times 4$ matrix

$$
V=\left(\begin{array}{cccc}
0 & K_{1} & K_{2} & K_{3} \\
-K_{1} & 0 & J_{3} & -J_{2} \\
-K_{2} & -J_{3} & 0 & J_{1} \\
-K_{3} & J_{2} & -J_{1} & 0
\end{array}\right)
$$

which satisfies

$$
\left[V^{\mu \nu}, V^{\rho \sigma}\right]=i\left(g^{\nu \rho} V^{\mu \sigma}-g^{\mu \rho} V^{\nu \sigma}-g^{\nu \sigma} V^{\mu \rho}+g^{\mu \sigma} V^{\nu \rho}\right) .
$$

In order to fully flesh out our relativistic invariance we still need to account for translations. This is fairly easily done through the introduction of generators for space and time translation: $P^{\mu}$. These generators obey the commutation relations

$$
\left[P_{\mu}, P_{\nu}\right]=0, \quad\left[V_{\mu \nu}, P_{\rho}\right]=i\left(g_{\mu \rho} P_{\nu}-g_{\nu \rho} P_{\mu}\right)
$$

Along with Eq. 2.19, these relations define the full Poincaré group.

There is one other interesting point that can be gleaned before moving on from generators. If we take our Lorentz group generators from Eq. 2.16 and combine them to form $J_{i}^{+}=\frac{1}{2}\left(J_{i}+i K_{i}\right)$ and $J_{i}^{-}=\frac{1}{2}\left(J_{i}-i K_{i}\right)$, these allow us to write Eq. 2.17 in the revealing form:

$$
\left[J_{i}^{+}, J_{j}^{+}\right]=i \epsilon_{i j k} J_{k}^{+}, \quad\left[J_{i}^{-}, J_{j}^{-}\right]=i \epsilon_{i j k} J_{k}^{-}, \quad\left[J_{i}^{+}, J_{j}^{-}\right]=0 .
$$

This reveals that our Lorentz Lie algebra actually has two commuting subalgebras for $3 \mathrm{D}$ rotations. Or, in other words, if we complexify our Lorentz algebra $s u(1,3) \rightarrow$ 
$s u(1,3)_{\mathbb{C}}$ we can split it into two parts:

$$
s o(1,3)_{\mathbb{C}} \cong \operatorname{sl}(2, \mathbb{C}) \oplus \operatorname{sl}(2, \mathbb{C})
$$

Where $\operatorname{sl}(2, \mathbb{C})$ is the complexification of $s u(2)$. Thus, since we're dealing with $s u(2)$, we're dealing with the familiar form of spin from quantum mechanics. The above algebra indicates that we can characterize irreducible representations of the Lorentz group by two half-integers $X$ and $Y$ such that representation $(X, Y)$ will have $(2 X+$ 1) $(2 Y+1)$ spin states.

Now, before we go further down the group theory rabbit hole, let us take a brief interlude to discuss what we ultimately want out of representations of the Poincaré group. To do this, we need to think back on what we're actually interested in studying: particles. If we were to take a particle and do a Poincaré transformation, the momentum and spin projection would change in accordance with the transformation, but other quantum numbers and mass do not. This allows us to devise a rather odd definition of a particle: the set of states that mix only among themselves under the Poincaré group. In the language of group theory, particles transform under irreducible representations.

However, there is one more thing we want: unitarity. Within QFT, the most common task is to calculate matrix elements; these should be equal in any reference frame. So, under a Poincaré transformation:

$$
\mathcal{M}=\left\langle\phi_{f} \mid \phi_{i}\right\rangle \rightarrow \mathcal{M}=\left\langle\phi_{f}\left|\mathcal{P}^{\dagger} \mathcal{P}\right| \phi_{i}\right\rangle
$$

This requires $\mathcal{P}^{\dagger} \mathcal{P}=\mathbb{1}$ - the unitarity we desired. Thus, particles should transform under irreducible unitary representations of the Poincaré group. It should be noted 
that finite-dimensional unitary representations of this group do not exist; we require infinite dimensional representations!

An important feature of unitary irreducible representations of the Poincaré group was discovered by Wigner in the late 1930s [21]: representations of this type are uniquely defined by a non-negative real number $m$ (mass) and a non-negative half-integer $J$ (spin). Further, Wigner was able to demonstrate that for $J=0$ there is only one spin state of spin zero and that for $J>0$ there are two scenarios: 1) the massless case, $m=0$, where the spin can be $\pm J$ for a total of two states and 2) the massive case, $m>0$, where there are spin states at $-J$ and $J$ and at every integer step between for a total of $2 J+1$ states. Unsurprisingly, this matches our understanding of spin from quantum mechanics.

\subsection{The Lagrangians of quantum fields}

With the rules of spacetime outlined, our task shifts to determining how to embed particles into fields and the resulting form of the Lagrangians of these fields. The $\mathrm{SM}$ is filled with a collection of both massless and massive particles with spins of $0, \frac{1}{2}$, or 1 (of course, composite particles can have larger spins and gravitons are spin-2 particles, but these are not part of the SM and are beyond the scope of this work). As such, in order to write down our full Poincaré invariant theory the objects used to describe the particle states of various spin must fit into our appropriately transforming fields: scalars $\phi(x)$, vectors $V^{\mu}(x)$, rank-2 tensors $T^{\mu \nu}$, spinors $\psi(x)$, and so on. Immediately, an interesting challenge becomes apparent: a massive spin-1 has 3 degrees of freedom, while the smallest object that can hold it has 4 . These extra degrees of freedom can lead to additional requirements being levied on the fields gauge invariance being one important example. 
The simplest case is for spin- 0 particles. They feature only one degree of freedom and thus can be represented by a scalar field $\phi(x)$. The free Lagrangian for a single, real scalar is simple:

$$
\mathcal{L}=\frac{1}{2}\left(\partial_{\mu} \phi\right)\left(\partial^{\mu} \phi\right)-\frac{m^{2}}{2} \phi^{2}
$$

including only bilinear (kinetic) terms. Applying Eqn. 2.5 gives us the famous equation of motion, known as the Klein-Gordon equation:

$$
\partial_{\mu} \partial^{\mu} \phi+m^{2} \phi=\left(\square+m^{2}\right) \phi=0
$$

This isn't a particularly interesting theory, as it lacks interaction terms like $a \phi^{3}$ or $b \phi^{4}$ (we restrict Lagrangian terms to having a mass dimension of $\leq 4$ to maintain renormalizability of the theory, so terms like $\lambda \phi^{7}$ are not permitted); I'll return to these in a bit. In the standard model, the Higgs boson is a spin-0 scalar and plays a critical role in gifting mass to other particles - more on this in Sec. 2.7.

Moving on to massive spin-1 states, three degrees of freedom need to be embedded so we must make use of a vector field $A^{\mu}(x)$. Of course, this has four components: indicating that this four-dimensional representation of the Lorentz group is made up of the direct sum of a one dimensional spin- 0 and a three dimensional spin- 1 component of $S O(3)$. Explicitly, under the Eq. 2.22 algebra real 4-vectors are acted on by a $\left(\frac{1}{2}, \frac{1}{2}\right)$ Lorentz representation. In order to ensure that we maintain explicit relativistic invariance and remove the additional degree of freedom, a gauge must be chosen. Taking the Lorentz gauge: $\partial_{\mu} A^{\mu}=0$ removes the extra degree of freedom and leads to the free Lagrangian for massive spin-1 particles, the Proca Lagrangian

$$
\mathcal{L}=-\frac{1}{4} F_{\mu \nu}^{2}+\frac{1}{2} m^{2} A_{\mu}^{2}
$$


with $F_{\mu \nu}=\partial_{\mu} A_{\nu}-\partial_{\nu} A_{\mu}$ being the field strength tensor. The equations of motion resulting from Eq. 2.26 are $\left(\square+m^{2}\right) A_{\mu}=0$ and $\partial_{\mu} A^{\mu}=0$. Solving these equations leads to the introduction of polarization vectors: three 4 -vectors $\epsilon_{\mu}^{i}(p)$ ensure solutions $A_{\mu}(x)$ that satisfy the first equation of motion also satisfy the second. Physically, this manifests as three polarization states for a massive vector particle: two transverse and one longitudinal. In the SM, the $W$ and $Z$ bosons are massive spin-1 particles and, as expected, they feature three polarization modes.

Turning to the massless vector case, the Proca Lagrangian in Eq. 2.26 reduces to

$$
\mathcal{L}=-\frac{1}{4} F_{\mu \nu}^{2}
$$

in the massless limit. According to Wigner's classification, massless spin-1 particles should have two degrees of freedom. The restrictions on $A^{\mu}(x)$ come from a new property that wasn't present in the massive case: the transformation

$$
A_{\mu}(x) \rightarrow A_{\mu}(x)+\partial_{\mu} \alpha(x) .
$$

leaves Eq. 2.27 invariant; this is referred to as gauge invariance and holds for any function $\alpha(x)$. Eq. 2.28 can be used to gauge-fix our solutions to the equations of motion, imposing constraints on $A_{\mu}(x)$ such that two degrees of freedom are removed, leaving the expected two polarizations for the massless vector bosons. These polarizations are transverse, matching our observation of one of the SM's massless spin-1 particles: the photon.

Before moving on to explore fermions and all the joys and sorrows that spinors have to offer, it is valuable to look beyond the free field theories introduced thus far and examine a simple interacting theory — scalar quantum electrodynamics (QED) 
- in order to demonstrate the central ideas of covariant derivatives, gauge-kinetic interactions, and conserved currents. Scalar QED features a photon $A_{\mu}$ and a complex scalar $\phi$ and its conjugate $\phi^{\star}$ (this can be thought of as two real scalar fields $\phi_{1}$ and $\phi_{2}$ combining as $\phi=\phi_{1}+i \phi_{2}$ and $\left.\phi^{\star}=\phi_{1}-i \phi_{2}\right)$. The free real scalar Lagrangian of Eq 2.24 can easily be generalized to

$$
\mathcal{L}=\left|\partial_{\mu} \phi\right|^{2}-m^{2} \phi^{\star} \phi
$$

Introducing a gauge transformation

$$
\phi(x) \rightarrow e^{-i \alpha(x)} \phi(x)
$$

leaves the mass term invariant but changes the kinetic term due to the derivatives. Fortunately, this set-back can be worked around fairly easily. Putting a (conventional) constant into the gauge transformation of the photon field (Eq. 2.28) such that $A_{\mu} \rightarrow$ $A_{\mu}+\frac{1}{e} \partial_{\mu} \alpha$ makes

$$
\left(\partial_{\mu}+i e A_{\mu}\right) \phi(x) \rightarrow\left(\partial_{\mu}+i e A_{\mu}+i \partial_{\mu} \alpha\right) e^{-i \alpha(x)} \phi(x)=e^{-i \alpha(x)}\left(\partial_{\mu}+i e A_{\mu}\right) \phi(x)
$$

Thus, we define the covariant derivative:

$$
D_{\mu} \phi \equiv\left(\partial_{\mu}+i e A_{\mu}\right) \phi \rightarrow e^{-i \alpha(x)} D_{\mu} \phi(x)
$$

With this, we can write down a fully gauge invariant Lagrangian for our scalar QED theory

$$
\mathcal{L}=-\frac{1}{4} F_{\mu \nu}^{2}+\left|D_{\mu} \phi\right|^{2}-m^{2} \phi^{\star} \phi
$$

On top of being gauge invariant, the gauge-kinetic $\left(D_{\mu}\right)$ term also encodes interaction 
terms between the scalars and the photon — giving our theory a breath of life

$$
\mathcal{L}_{i n t}=i e\left(\phi \partial_{\mu} \phi^{\star}-\phi^{\star} \partial_{\mu} \phi\right) A^{\mu}
$$

Even more interesting is that gauge symmetries imply global symmetries which by Noether's theorem (explored in Sec. 2.2) imply a conserved current or conserved charge. For scalar QED, the symmetry of the gauge transformation is $U(1)$ and the conserved charge is electric charge: $\phi$ carries charge $-e$ and $\phi^{\star}$ carries charge $e$.

Gauge symmetries can arise that have more complicated structures than the $U(1)$ group. Most notably the SM features $S U(2)_{L}$ and $S U(3)_{C}$ which are both nonabeliean; more on this in Sec. 2.5. For now, we need to direct our attention towards the particles that make up a majority of the everyday world around us: fermions.

Spin- $\frac{1}{2}$ particles possess two degrees of freedom and can have a Lorentz representation of either $\left(\frac{1}{2}, 0\right)$ or $\left(0, \frac{1}{2}\right)$. Determining $2 \times 2$ matrices that satisfy the generator commutation relations of Eq. 2.21 leads us back to the familiar Pauli matrices

$$
\left(\begin{array}{ll}
0 & 1 \\
1 & 0
\end{array}\right), \quad\left(\begin{array}{cc}
0 & -i \\
i & 0
\end{array}\right), \quad\left(\begin{array}{cc}
1 & 0 \\
0 & -1
\end{array}\right) .
$$

The Pauli matrices obey an $S U(2)$ algebra

$$
\left[\frac{\sigma_{i}}{2}, \frac{\sigma_{j}}{2}\right]=i \epsilon_{i j k} \frac{\sigma_{k}}{2}
$$

Returning to our Lorentz representations, we can write out the generators:

$$
\left(\frac{1}{2}, 0\right): \quad \vec{J}^{-}=\frac{\vec{\sigma}}{2}, \quad \vec{J}^{+}=0 \quad \text { and } \quad\left(0, \frac{1}{2}\right): \quad \vec{J}^{-}=0, \quad \vec{J}^{+}=\frac{\vec{\sigma}}{2}
$$


Of course, if we want, we can obtain the actual Lorentz transformations (rotations and boosts) through the application of the definitions of $J_{i}^{+}=\frac{1}{2}\left(J_{i}+i K_{i}\right)$ and $J_{i}^{-}=\frac{1}{2}\left(J_{i}-i K_{i}\right)$. It also should be noted that the group generators of these two representations $\left(\left(\frac{1}{2}, 0\right)\right.$ and $\left.\left(0, \frac{1}{2}\right)\right)$ are adjoints of each other. More important, however, is the identification of the objects in the $2 \mathrm{D}$ vector space acted on by these representations: spinors. Spinors that are acted on by the $\left(\frac{1}{2}, 0\right)$ representation are left-handed Weyl spinors (denoted with a subscript "L", $\psi_{L}$ ) whereas those acted on by the $\left(0, \frac{1}{2}\right)$ representation are right-handed (denoted with a subscript "R", $\psi_{R}$ ).

With Weyl spinors in hand, our task again becomes one of constructing valid Lagrangians that obey the symmetries of spacetime. Terms of the form $\psi_{R}^{\dagger} \psi_{R}$ turn out to not be Lorentz invariant, making the simplest Lorentz invariant term be a combination of bilinears

$$
\mathcal{L}_{\text {Dirac mass }}=m\left(\psi_{L}^{\dagger} \psi_{R}+\psi_{R}^{\dagger} \psi_{L}\right)
$$

referred to as the Dirac mass. Adding in kinetic terms (again requiring Lorentz invariance) leads to the Lagrangian

$$
\mathcal{L}=i \psi_{R}^{\dagger} \sigma_{\mu} \partial_{\mu} \psi_{R}+i \psi_{L}^{\dagger} \bar{\sigma}_{\mu} \partial_{\mu} \psi_{L}-m\left(\psi_{L}^{\dagger} \psi_{R}+\psi_{R}^{\dagger} \psi_{L}\right)
$$

where $\sigma_{\mu} \equiv(\mathbb{1}, \vec{\sigma})$ and $\bar{\sigma}_{\mu} \equiv(\mathbb{1},-\vec{\sigma})$. Combining the two spinors into a fourcomponent Dirac spinor

$$
\psi=\left(\begin{array}{l}
\psi_{L} \\
\psi_{R}
\end{array}\right)
$$


and defining $\bar{\psi} \equiv \psi^{\dagger} \gamma^{0}$ allows us to write the Lagrangian in a more familiar way

$$
\mathcal{L}=\bar{\psi}\left(i \gamma^{\mu} \partial_{\mu}-m\right) \psi
$$

This Dirac Lagrangian's equation of motion is the famous Dirac equation

$$
\left(i \gamma^{\mu} \partial_{\mu}-m\right) \psi=0
$$

The $\gamma$-matrices in Eq. $2.41 \& 2.42$ are the matrix representations of a Clifford algebra defined by the anti-commutation relation

$$
\left\{\gamma^{\mu}, \gamma^{\nu}\right\}=2 g^{\mu \nu}
$$

There are several representations of $\gamma$-matrices that can be used. For our purposes this choice is of little consequence; the relevant calculations can be completed through the use of a collection of matrix and trace identities. Three more useful objects can be defined, the $\gamma^{5}$ operator

$$
\gamma^{5} \equiv i \gamma^{0} \gamma^{1} \gamma^{2} \gamma^{3}
$$

slash notation

$$
\not p \equiv \gamma^{\mu} p_{\mu}
$$

and the projection operators $P_{L}$ and $P_{R}$ which respectively separate out the left and right-handed components of a Dirac spinor

$$
P_{L} \equiv \frac{1}{2}\left(1-\gamma^{5}\right) \quad P_{R} \equiv \frac{1}{2}\left(1+\gamma^{5}\right)
$$

With the spinor framework in place, we return to gauge transformation. Taking 
a spinor with charge $\mathrm{Q}=-1$ (as is the case for an electron) the gauge transformation is

$$
\psi \rightarrow e^{-i \alpha} \psi
$$

This is identical to the scalar case presented in Eq. 2.30 indicating that we can use the same covariant derivative found in Eq. 2.32: $D_{\mu}=\partial_{\mu}+i e A_{\mu}$. Just as in the scalar QED case, we now have everything we need to write down the full regular QED Lagrangian

$$
\mathcal{L}=-\frac{1}{4} F_{\mu \nu}^{2}+i \bar{\psi} \not D_{\mu} \psi-m \bar{\psi} \psi
$$

Finally, we have a theory that describes our universe (or a part of it, at the very least)! As the name suggests, quantum electrodynamics is the relativistic quantum field theory of electrodynamics; it is in full agreement with both special relativity and quantum mechanics and describes all phenomena involving electrically charged particles interacting via photons. Formally, this theory features the same $U(1)$ gauge invariance as scalar QED. This global symmetry leads to a Noether current of $J_{\mu}=$

$\bar{\psi} \gamma_{\mu} \psi$ and a conserved charge $Q$ that represents electron number - this is related to the total electric charge through the coupling constant $e$.

\subsection{Non-Abelian gauge theories}

Although the effectiveness and accuracy of QED is incredibly difficult to understate, the type of theories that we can write down with the tools developed thus far are much too limited to describe all of the particles we see in nature. Perhaps most notably, the self-interactions that occur among gluons are completely absent — making a description of the strong force impossible.

Rather than diving off the deep end and looking directly at the QCD Lagrangian 
it helps to look at Yang-Mills theories: these are generalizations of QED that feature multiple massless spin-1 particles that interact with each other. The gauge invariance present in these theories differs from QED in a critical way, as we must move from the Abelian QED to non-Abelian Yang-Mills.

In the simplest case, we can imagine that we have a theory with two scalars $\phi_{1}$ and $\phi_{2}$ such that their kinetic Lagrangian terms have the form:

$$
\mathcal{L}_{k i n}=\left(\partial_{\mu} \phi_{1}^{\star}\right)\left(\partial^{\mu} \phi_{1}\right)+\left(\partial_{\mu} \phi_{2}^{\star}\right)\left(\partial^{\mu} \phi_{2}\right)=\left(\partial_{\mu} \vec{\phi}\right)^{\dagger}\left(\partial^{\mu} \vec{\phi}\right)
$$

with $\vec{\phi}=\left(\begin{array}{ll}\phi_{1} & \phi_{2}\end{array}\right)^{T}$. Interestingly, Eq 2.49 is invariant under a global $S U(2)$ symmetry $(2 \times 2$ unitary matrix with determinant 1$) \vec{\phi} \rightarrow U \vec{\phi}^{1}$. In full generality $U$ can be written

$$
U=e^{i \alpha^{a} \tau^{a}}
$$

where $\alpha^{a}$ are numbers and $\tau^{a}$ are the $S O(3)$ generators from Eq. 2.36 that are built from the Pauli matrices. This global symmetry can be promoted to a local symmetry by making $\alpha^{a}$ into functions of spacetime $\alpha^{a}(x)$. Under this local symmetry, we must replace the standard derivatives with covariant derivatives

$$
D_{\mu} \vec{\phi}=\partial_{\mu} \vec{\phi}-i g A_{\mu}^{a} \tau^{a} \vec{\phi}
$$

with a coupling strength of $g$. Although this is similar to the covariant derivative of the previous section (Eq. 2.32) there is one critical difference: there are now three

\footnotetext{
${ }^{1}$ Technically the invariance is under $U(2)=U(1) \times S U(2)$, but the important parts we need to discuss are in the $S U(2)$ subgroup.
} 
gauge bosons $A_{\mu}^{a}$ that transform as

$$
A_{\mu}^{a}(x) \rightarrow A_{\mu}^{a}(x)+\frac{1}{g} \partial_{\mu} \alpha^{a}(x)-f^{a b c} \alpha^{b}(x) A_{\mu}^{c}(x)
$$

$f^{a b c}$ are structure constants dependent on the underlying symmetry of the theory - in this case, $S U(2), f^{a b c}=\epsilon^{a b c}$ where $\epsilon^{a b c}$ is the Levi-Civita tensor. In the case of an Abelian gauge theory $f^{a b c}=0$ and we obtain the transformation of Eq. 2.28. Explicitly, this structure constant arises from the failure of the group generators to commute, making the gauge theory non-Abelian.

Returning to our particle fields, the non-Abelian symmetry requires a modification to the field strength tensor,

$$
F_{\mu \nu}^{a}=\partial_{\mu} A_{\nu}^{a}-\partial_{\nu} A_{\mu}^{a}+g f^{a b c} A_{\mu}^{b} A_{\nu}^{c}
$$

This can immediately be used to construct the Yang-Mills Lagrangian for these gauge bosons

$$
\mathcal{L}_{Y M}=-\frac{1}{4} F_{\mu \nu}^{a} F^{a \mu \nu}=\frac{1}{4} \sum_{a}\left(\partial_{\mu} A_{\nu}^{a}-\partial_{\nu} A_{\mu}^{a}+g f^{a b c} A_{\mu}^{b} A_{\nu}^{c}\right)^{2} .
$$

We have what we need, a gauge-invariant theory that features self-interactions! This has many implications, including the possibility of virtual gauge bosons producing vacuum polarization effects with opposite sign to scalars or spinors. This allows for an intriguing phenomenon that we see in Quantum Chromodynamics (QCD): forces can become stronger at long distances.

On a mathematical level, the SM gauge group is $S U(3)_{C} \otimes S U(2)_{L} \otimes U(1)_{Y}$ with an associated Lie algebra $s u(3) \oplus s u(2) \oplus u(1)$. This Lie algebra is semisimple — the direct sum of simple Lie algebras ${ }^{2}$. Theorems from Lie algebra demonstrate

\footnotetext{
${ }^{2}$ A simple Lie algebra has no non-trivial ideals, where an ideal is a subalgebra $\mathcal{I} \subset \mathcal{G}$ satisfying
} 
that all finite-dimensional representations of semisimple algebras are Hermitian and can thus be used in the construction of unitary theories.

Broadly speaking, there are four types of compact finite-dimensional simple Lie groups that are relevant to physics (there can be multiple groups that correspond to the same algebra that feature different forms):

1. $\mathbf{S U}(\mathbf{N})$, Special unitary groups: Groups that conserve a complex inner product $\langle\psi \mid \phi\rangle$ are unitary. In other words, group elements $U$ obey $U^{\dagger} U=\mathbb{1}$. The "special" refers to the fact that $\operatorname{det}(U)=1$. Finally, groups $S U(N)$ possess $N^{2}-1$ generators.

2. $\mathbf{S O}(\mathbf{N})$, Special orthogonal groups: Groups that preserve a real inner product $X \cdot Y$, so group elements $O$ obey $O^{T} O=\mathbb{1}$. $S O(N)$ has $\frac{N}{2}(N-1)$ generators.

3. $\mathbf{S p}(\mathbf{N})$, Symplectic groups: Groups that conserve a quaternionic inner product, group members $S$ obey $S^{T} \Omega S=\Omega$ for $\Omega=\left(\begin{array}{cc}0 & \mathbb{1} \\ -\mathbb{1} & 0\end{array}\right)$.

4. Exceptional groups: The groups $G_{2}, F_{4}, E_{6}, E_{7}$, and $E_{8}$ are the only other finite-dimensional simple Lie algebras.

With the allowed groups outlined, the challenge shifts to understanding how particles transform under these groups, especially in the cases of $S U(2)$ and $S U(3)$ that are found in the SM. It turns out that particles transform under the fundamental representation (and their complex conjugates under the anti-fundamental representation): this is the smallest non-trivial representation of an algebra. On the other hand, gauge fields transform in accordance with the adjoint representation, a representation that acts on the space spanned by the generators of the group. For $S U(N)$ $[g, i] \subset \mathcal{I}$ for any $g \in \mathcal{G}$ and $i \in \mathcal{I}$. 
the fundamental representation is made up $N \times N$ Hermitian matrices of determinant 1. If we have $N$ fields $\phi_{i}$, they transform following

$$
\phi_{i} \rightarrow \phi_{i}+i \alpha^{a}\left(T_{\text {fund }}^{a}\right)_{i j} \phi_{j}
$$

and their complex conjugates transform following the anti-fundamental representation

$$
\phi_{i}^{\star} \rightarrow \phi_{i}^{\star}+i \alpha^{a}\left(T_{a n t i-f u n d}^{a}\right)_{i j} \phi_{j}^{\star}
$$

where $T_{\text {anti-fund }}^{a}=-\left(T_{\text {fund }}^{a}\right)^{\star}$. For the SM the indices a refer to either colour (in the case of $S U(3))$ or isospin (for $S U(2)$ ). We've already seen generators for $S U(2)$ several times (Eq. $2.36 \& 2.50$ ) — they are the Pauli matrices divided by 2. For $S U(3)$ there are $3^{2}-1=8$ generators $T^{a}=\frac{1}{2} \lambda^{a}$ where $\lambda^{a}$ are the Gell-Mann matrices:

$$
\begin{aligned}
& \lambda_{1}=\left(\begin{array}{lll}
0 & 1 & 0 \\
1 & 0 & 0 \\
0 & 0 & 0
\end{array}\right), \lambda_{2}=\left(\begin{array}{ccc}
0 & -i & 0 \\
i & 0 & 0 \\
0 & 0 & 0
\end{array}\right), \lambda_{3}=\left(\begin{array}{ccc}
1 & 0 & 0 \\
0 & -1 & 0 \\
0 & 0 & 0
\end{array}\right), \lambda_{4}=\left(\begin{array}{ccc}
0 & 0 & 1 \\
0 & 0 & 0 \\
1 & 0 & 0
\end{array}\right), \\
& \lambda_{5}=\left(\begin{array}{lll}
0 & 0 & -i \\
0 & 0 & 0 \\
i & 0 & 0
\end{array}\right), \lambda_{6}=\left(\begin{array}{lll}
0 & 0 & 0 \\
0 & 0 & 1 \\
0 & 1 & 0
\end{array}\right), \lambda_{7}=\left(\begin{array}{ccc}
0 & 0 & 0 \\
0 & 0 & -i \\
0 & i & 0
\end{array}\right), \lambda_{8}=\frac{1}{\sqrt{3}}\left(\begin{array}{ccc}
1 & 0 & 0 \\
0 & 1 & 0 \\
0 & 0 & -2
\end{array}\right) .
\end{aligned}
$$

As for the adjoint representations, these are $N^{2}-1$ (the number of generators) dimensional representations for $S U(N)$ defined by $\left(T_{a d j}^{a}\right)^{b c}=-i f^{a b c}$. Thus, for $S U(2)$ we have 


$$
T_{a d j}^{1}=\left(\begin{array}{ccc}
0 & 0 & 0 \\
0 & 0 & -i \\
0 & i & 0
\end{array}\right) T_{a d j}^{2}=\left(\begin{array}{ccc}
0 & 0 & i \\
0 & 0 & 0 \\
-i & 0 & 0
\end{array}\right) T_{a d j}^{3}=\left(\begin{array}{ccc}
0 & -i & 0 \\
i & 0 & 0 \\
0 & 0 & 0
\end{array}\right)
$$

defining how the gauge fields transform.

As a final point before moving on to the full field content of the SM, it is important to keep in mind that all the discussions of Lie groups and representations are still rooted in the idea of symmetry and further that these symmetries result in Noether currents and conserved charges. Ultimately, this gives us a collection of conservation laws from Poincaré invariance including conservation of energy and momentum. The internal gauge symmetries of the Lagrangian lead to additional conserved charges like electric charge ${ }^{3}$ and colour. These symmetries of the Standard Model are summarized in Table 2.1.

\subsection{Field content of the Standard Model}

With all the machinery built up over the last several sections, it is time to tackle the full SM and lay out all the particles, symmetries, and interactions. The full SM

\footnotetext{
${ }^{3}$ Technically weak hypercharge is conserved pre-symmetry breaking.
}

\begin{tabular}{|c|c|}
\hline Conservation Law & Noether Symmetry Invariance \\
\hline Conservation of energy & Time-translation invariance \\
\hline Conservation of momentum & Space-translation invariance \\
\hline Conservation of angular momentum & Rotation invariance \\
\hline Conservation of CM velocity & Boost invariance \\
\hline Conservation of electric charge & $U(1)$ gauge invariance \\
\hline Conservation of weak isospin & $S U(2)$ gauge invariance \\
\hline Conservation of colour charge & $S U(3)$ gauge invariance \\
\hline
\end{tabular}

Table 2.1: Symmetries of the Standard Model. 
Lagrangian can be broken up into various parts

$$
\mathcal{L}_{S M}=\mathcal{L}_{\text {gauge }}+\mathcal{L}_{\text {FermionGK }}+\mathcal{L}_{H}+\mathcal{L}_{\text {Yukawa }}
$$

that we will explore in turn.

As mentioned in the previous section, the SM gauge group is $S U(3)_{C} \times S U(2)_{L} \times$ $U(1)_{Y}$; from this we immediately expect $N^{2}-1$ generators for the two $S U(N)$ groups and 1 for $U(1)_{Y}$. This immediately suggests that we should have 8, 3, and 1 gauge bosons that live in the adjoint representations of their respective groups (see Eq. 2.58). Each of these groups will have their own coupling constant, which we denote $g_{s}, g$, and $g^{\prime}$ for $S U(3)_{C}, S U(2)_{L}$, and $U(1)_{Y}$, respectively. We can package the gauge fields $G_{\mu}^{b}, W_{\mu}^{a}$, and $B_{\mu}$ in field strength tensors $G_{\mu \nu}^{b}, W_{\mu \nu}^{a}$, and $B_{\mu \nu}$ using Eq. 2.53: the structure constants are chosen according to the symmetry of the corresponding group. Ultimately, this gives the first part of the SM Lagrangian, the gauge field tensors

$$
\mathcal{L}_{\text {gauge }}=-\frac{1}{4} G_{\mu \nu}^{b} G^{b \mu \nu}-\frac{1}{4} W_{\mu \nu}^{a} W^{a \mu \nu}-\frac{1}{4} B_{\mu \nu} B^{\mu \nu} .
$$

With the gauge structure of the SM in place, we can now write down all SM particles that we see in nature. We classify these into particle multiplets according to how they transform under the symmetries of the SM gauge group — or, in other words, how they interact with the gauge fields. Since there are three gauge symmetries present, we can denote the transformation properties of a multiplet using $(\mathbf{C}, \mathbf{L}, Y)$ : $\mathbf{C}$ is the representation of the multiplet under $S U(3)_{C}, \mathbf{L}$ is the representation under $S U(2)_{L}$, and $Y$ is the weak hypercharge. In its entirety, the field content of the SM is: 
1. $G_{\mu}^{b}$ : The gauge fields for the strong force $S U(3)_{C}-$ spin-1 gluons. Possesses $(\mathbf{8}, \mathbf{1}, 0)$ gauge charges.

2. $W_{\mu}^{a}$ : The gauge fields for weak isospin $S U(2)_{L}-$ spin-1 $\mathrm{W}$ bosons. Possesses $(\mathbf{1}, \mathbf{3}, 0)$ gauge charges.

3. $B_{\mu}$ : The gauge field for weak hyperchrage $U(1)_{Y}-$ the $\mathrm{B}$ boson. Possesses $(\mathbf{1}, \mathbf{1}, 0)$ gauge charges.

4. $Q_{L}^{m}$ : Left-handed spin- $\frac{1}{2}$ quark doublet. Contains the left-handed up and down quarks. Possesses $\left(\mathbf{3}, \mathbf{2}, \frac{1}{6}\right)$ gauge charges.

5. $u_{R}^{m}$ : Right-handed spin- $\frac{1}{2}$ quark singlet. Contains the right-handed up quark. Possesses $\left(\mathbf{3}, \mathbf{1}, \frac{2}{3}\right)$ gauge charges.

6. $d_{R}^{m}$ : Right-handed spin- $\frac{1}{2}$ quark singlet. Contains the right-handed down quark. Possesses $\left(\mathbf{3}, \mathbf{1}, \frac{-1}{3}\right)$ gauge charges.

7. $L_{L}^{m}$ : Left-handed spin- $\frac{1}{2}$ lepton doublet. Contains the left-handed electron and neutrino. Possesses $\left(\mathbf{1}, \mathbf{2}, \frac{-1}{2}\right)$ gauge charges.

8. $e_{R}^{m}$ : Right-handed spin- $\frac{1}{2}$ lepton singlet. Contains the right-handed electron. Possesses $(\mathbf{1}, \mathbf{1},-1)$ gauge charges.

9. $H$ : The higgs scalar doublet. Possesses $\left(\mathbf{1}, \mathbf{2}, \frac{1}{2}\right)$ gauge charges.

In light of the discussion in Sec. 2.5, the results are unsurprising: particles that are charged under a non-abelian gauge group transform in the fundamental representation, particles that are not transform under the trivial representation, and gauge fields transform under the adjoint representation. Each of the fermion multiplets features three different flavour generations (denoted by the superscript $m$ ) that contain 
successively heavier particles. The lepton generations, for example, consist of the electron, muon, and tau particles as well as their corresponding neutrinos; the quark generations feature up, charm, and top up-type quarks with charge $2 / 3$ and down, strange, and bottom down-type with charge $-1 / 3$. The ratio of the masses between generations can be quite significant — this is especially notable in the case of the top quark which has a mass of $\sim 173 \mathrm{GeV}$ versus the $\sim 1.3 \mathrm{GeV}$ for the charm quark [22]. Finally, each generation is a fully anomaly free gauge theory. The field content of the Standard model is summarized in Table 2.2.

The SM covariant derivative is the natural extension of those used in QED and Yang-Mills (Eq. 2.32 \& 2.51) and includes terms for each gauge symmetry

$$
D_{\mu}=\partial_{\mu}-i g^{\prime} B_{\mu} Y-i g W_{\mu}^{a} \tau_{a}-i g_{s} G_{\mu}^{b} \lambda_{b}
$$

with $\lambda_{b}$ and $\tau_{a}$ being the generators outlined in the previous section. With this

\begin{tabular}{|c|c|c|c|c|}
\hline & $S U(3)_{C}$ & $S U(2)_{L}$ & $U(1)_{Y}$ & Spin \\
\hline$H$ & $\mathbf{1}$ & $\mathbf{2}$ & $1 / 2$ & 0 \\
\hline$Q_{L}^{m}$ & $\mathbf{3}$ & $\mathbf{2}$ & $1 / 6$ & $1 / 2$ \\
\hline$u_{R}^{m}$ & $\mathbf{3}$ & $\mathbf{1}$ & $2 / 3$ & $1 / 2$ \\
\hline$d_{R}^{m}$ & $\mathbf{3}$ & $\mathbf{1}$ & $-1 / 3$ & $1 / 2$ \\
\hline$L_{L}^{m}$ & $\mathbf{1}$ & $\mathbf{2}$ & $-1 / 2$ & $1 / 2$ \\
\hline$e_{R}^{m}$ & $\mathbf{1}$ & $\mathbf{1}$ & -1 & $1 / 2$ \\
\hline$B_{\mu}$ & $\mathbf{1}$ & $\mathbf{1}$ & 0 & 1 \\
\hline$W_{\mu}^{a}$ & $\mathbf{1}$ & $\mathbf{3}$ & 0 & 1 \\
\hline$G_{\mu}^{b}$ & $\mathbf{8}$ & $\mathbf{1}$ & 0 & 1 \\
\hline
\end{tabular}

Table 2.2: Field content of the Standard Model. The flavour index $m$ goes from 1 to 3 and indicates the generation of the fermions. $a$ from 1 to 3 and $b$ from 1 to 8 , indicating the gauge bosons for $S U(2)$ and $S U(3)$, respectively. The $L$ and $R$ subscripts for the fermion multiplets represent the multiplet's chirality. The numbers in the $S U(3)_{C}$ and $S U(2)_{L}$ columns show the representation of the various multiplets under the respective symmetry; the $U(1)_{Y}$ column denotes the hypercharge of the multiplets. 
covariant derivative, we can construct gauge-kinetic terms for the various fermionic multiplets in a similar way to Eq. 2.48

$$
\mathcal{L}_{\text {Fermion } G K}=\sum_{\Psi} i \bar{\Psi} \not D \Psi
$$

$\Psi$ represents Dirac spinors of the fermion multiplets and all their generations. It should be noted that projection operators can be used on this Dirac spinor to obtain the left and right-handed chiral fermion states. For example, we can embed the first lepton generation into a Dirac spinor such that $P_{L} \Psi=\Psi_{L}=L_{L}^{1}$.

With just the gauge fields and the fermionic multiplets, this is as far as we can go. All the gauge bosons must be massless as terms such as

$$
\mathcal{L} \supset \frac{1}{2} m^{2} B_{\mu} B^{\mu}
$$

are not gauge invariant and thus cannot be freely inserted into the Lagrangian. Similarly, looking back to Eq. 2.39 demonstrates that mass terms for the fermionic multiplets would require terms of the form

$$
\mathcal{L} \supset-m\left(\bar{\psi}_{L} \psi_{R}+\bar{\psi}_{R} \psi_{L}\right)
$$

Since these terms involve fermions of both chiralities, which both carry different gauge charges, these terms are not gauge invariant and the fermions must also be massless. Finally, we turn to the last multiplet of our theory, the Higgs scalar doublet $H$. It possesses Lagrangian terms of the form

$$
\mathcal{L}_{H}=\left(D_{\mu} H\right)^{\dagger}\left(D^{\mu} H\right)-V(H)
$$


with the scalar potential being defined as

$$
V(H)=-\mu^{2} H^{\dagger} H+\lambda\left(H^{\dagger} H\right)^{2}
$$

The form of this potential ends up having supreme importance as it allows for spontaneous symmetry breaking (to be explored in Sec. 2.7). For now, we just note that the Higgs has interaction with the gauge bosons through the covariant derivative.

Finally, we arrive at the last piece of the puzzle: the Yukawa terms. These terms describe permitted interactions between scalars and fermions that have the form

$$
\mathcal{L} \supset y \phi \bar{\psi} \psi
$$

where $y$ is a dimensionless coupling constant. Explicitly:

$$
\mathcal{L}_{Y u k a w a}=-y_{e} \bar{e}_{R} H^{\dagger} L_{L}-y_{u} \bar{u}_{R} \tilde{H}^{\dagger} Q_{L}-y_{d} \bar{d}_{R} H^{\dagger} Q_{L}+\text { h.c. }
$$

where $y_{X}$ are the Yukawa couplings and $\tilde{H} \equiv i \sigma^{2} H^{\star}$.

With our SM Lagrangian fully constructed, we can see that there are an additional 4 accidental symmetries that are present, despite not being postulated prior to the Lagrangian's construction. These 4 symmetries are all continuous global $U(1)$ phase symmetries of the form $E_{L} \rightarrow e^{i \alpha} E_{L}$ and $\bar{e}_{R} \rightarrow e^{-i \alpha} \bar{e}_{R}$. Each of these phases leads to conservation law, ultimately resulting in conservation of Baryon number, Electron number, Muon number, and Tau number. In addition, there are several approximate symmetries of the SM such as $S U(2)$ custodial symmetry and $S U(3)$ quark flavour symmetry. However, as these symmetries are of little relevance to the rest of this work, we will not discuss them any further. 


\subsection{The Higgs and electroweak symmetry break- ing}

The SM is now fully constructed yet things don't look quite how we would expect them to: fermions and gauge bosons are all massless - apparently throwing the theory into direct conflict with observable reality where most particles do have mass. In order to rectify this issue we need to turn to the concept of spontaneous symmetry breaking: a phenomenon where the lowest-energy vacuum solutions of a theory break a symmetry of the Lagrangian. For the SM, this occurs due to the presence of the higgs complex scalar doublet $H$ which we can express in the form

$$
H=\left(\begin{array}{c}
\phi^{+} \\
\phi^{0}
\end{array}\right)=\frac{1}{\sqrt{2}}\left(\begin{array}{c}
\phi^{1}+i \phi^{2} \\
\phi^{3}+i \phi^{4}
\end{array}\right)
$$

where the $\phi^{i}$ fields in the rightmost term are normalized real scalar fields.

Returning to the scalar potential of the higgs outlined in Eq. 2.66, $V(H)=$ $-\mu^{2} H^{\dagger} H+\lambda\left(H^{\dagger} H\right)^{2}$, it is clear that the behaviour of this potential is completely dependent on the signs of the constants $-\mu^{2}$ and $\lambda$. If $\lambda$ is negative, there is no stable vacuum state as the potential is unbounded from below — which is clearly not the case for our universe. If $\lambda$ is positive then there are two scenarios: 1) $-\mu^{2}$ is positive, which leads to minimum at $|H|=0$ and the preservation of electroweak symmetry, and 2) $-\mu^{2}$ is negative which leads to a minimum away from the origin. In the latter case, $S U(2) \times U(1)$ electroweak symmetry is not respected by the vacuum state, making the symmetry spontaneously broken [13].

The symmetry breaking case and its implications is the only one that has any hope of constructing a theory that matches nature, so we proceed to find the minimum 
of the potential

$$
H^{\dagger} H=\frac{\mu^{2}}{2 \lambda}
$$

In 4-dimensional field space (with coordinates $\phi^{i}$ ), this is the equation for a spherical shell in four dimensions, indicating that one can remain at the minimum of the potential while undergoing rotations in three dimensions (note that this corresponds to $S U(2) \times U(1)$ gauge transformations that leave the overall potential unchanged but transform the vacuum state by adjusting the direction) and, as such, these rotations cost zero energy. On the other hand, movement along the radial direction does cost energy and the potential behaves like that of a harmonic oscillator. From a particle perspective, we anticipate 3 Goldstone modes from the flat directions of the potential and 1 massive scalar from the radial direction.

Orienting the basis of states $\phi^{i}$ in a convenient way gives us vacuum expectation values (VEVs)

$$
\left\langle\phi_{3}\right\rangle=\sqrt{\frac{\mu^{2}}{\lambda}}, \quad\left\langle\phi_{1}\right\rangle=\left\langle\phi_{2}\right\rangle=\left\langle\phi_{4}\right\rangle=0 .
$$

We can further rewrite $\phi_{3}$ in a more useful form by defining a new real scalar field $h$ such that $\langle h\rangle=0, \phi_{3} \equiv h+v$ where $v$ is referred to as the SM VEV. This leads to a new form of Eq. 2.69

$$
H=\frac{1}{\sqrt{2}}\left(\begin{array}{c}
\phi^{1}+i \phi^{2} \\
h+v+i \phi^{4}
\end{array}\right) .
$$

Plugging this into the potential Eq. 2.66 indicates that the three Goldstone modes are massless and that $m_{h}=\sqrt{2 \lambda v^{2}}$, as expected. However, there is an even more useful form of the higgs doublet that can be reached through the application of a 
$S U(2) \times U(1)$ gauge transformations on $H$ giving the unitary gauge result

$$
H=\frac{1}{\sqrt{2}}\left(\begin{array}{c}
0 \\
h+v
\end{array}\right) .
$$

The fields $\phi_{1}, \phi_{2}$ and $\phi_{4}$ have been gauged away indicating that these fields, postsymmetry breaking, are not physical.

Now that the higgs doublet is in a much more workable state, we can explore the interactions and implications of the higgs particle $h$ on the rest of the SM. The self interaction of the higgs arise immediately by using the unitary gauge higgs doublet from Eq. 2.73 in the higgs potential 2.66

$$
\mathcal{L}_{S M} \supset-\lambda v^{2} h^{2}-\lambda v h^{3}-\frac{1}{4} \lambda h^{4}
$$

Slightly more complicated are the interactions between the higgs and gauge bosons which are taken from the gauge-kinetic part of the higgs Lagrangian Eq. 2.65.

$D_{\mu} H=\left(\partial_{\mu}-i g^{\prime} B_{\mu} Y-i g W_{\mu}^{a} \tau_{a}-i g_{s} G_{\mu}^{b} \lambda_{b}\right) H=\frac{1}{\sqrt{2}}\left(\begin{array}{c}-i \frac{g}{2}\left(W_{\mu}^{1}-i W_{\mu}^{2}\right)(v+h) \\ \partial_{\mu} h+i \frac{1}{2}\left(g W_{\mu}^{3}-g^{\prime} B_{\mu}\right)(v+h)\end{array}\right)$

leads to

$$
\left(D_{\mu} H\right)^{\dagger}\left(D^{\mu} H\right)=\frac{1}{2} \partial_{\mu} h \partial^{\mu} h+\frac{1}{8}(v+h)^{2}\left[g^{2}\left|W_{\mu}^{1}-i W_{\mu}^{2}\right|^{2}+\left(g W_{\mu}^{3}-g^{\prime} B_{\mu}\right)^{2}\right]
$$


Four substitutions can help us make a bit of sense of the above expression

$$
\begin{aligned}
W_{\mu}^{+} & =\frac{1}{\sqrt{2}}\left(W_{\mu}^{1}-i W_{\mu}^{2}\right), \\
W_{\mu}^{-} & =\frac{1}{\sqrt{2}}\left(W_{\mu}^{1}+i W_{\mu}^{2}\right), \\
Z_{\mu} & =\frac{1}{\sqrt{g+g^{\prime}}}\left(g W_{\mu}^{3}-g^{\prime} B_{\mu}\right)=\left(c_{W} W_{\mu}^{3}-s_{W} B_{\mu}\right), \\
A_{\mu} & =\frac{1}{\sqrt{g+g^{\prime}}}\left(g^{\prime} W_{\mu}^{3}+g B_{\mu}\right)=\left(s_{W} W_{\mu}^{3}+c_{W} B_{\mu}\right)
\end{aligned}
$$

where $s_{W}=\sin \theta_{W}$ and $c_{W}=\cos \theta_{W}$ are functions of the Weinberg angle $\theta_{W}$. Using these in Eq. 2.76 gives

$$
\begin{aligned}
\mathcal{L}_{H} & \supset \frac{g^{2} v^{2}}{4} W_{\mu}^{+} W^{-\mu}+\frac{g^{2} v}{2} h W_{\mu}^{+} W^{-\mu}+\frac{g^{2}}{4} h h W_{\mu}^{+} W^{-\mu} \\
& +\frac{\left(g^{2}+g^{\prime 2}\right) v^{2}}{8} Z_{\mu} Z^{\mu}+\frac{\left(g^{2}+g^{\prime 2}\right) v}{4} h Z_{\mu} Z^{\mu}+\frac{\left(g^{2}+g^{\prime 2}\right)}{8} h h Z_{\mu} Z^{\mu} .
\end{aligned}
$$

This is just what we needed! Three gauge bosons now have masses and interactions with the higgs: the $W$ bosons have $m_{W}=\frac{g v}{2}$ and the $Z$ boson has $m_{Z}=\frac{\sqrt{g^{2}+g^{\prime 2}} v}{2}$. The fourth gauge boson, which represents the photon, remains massless and free from any couplings to the higgs. The decay of higgs to two photons $h \rightarrow \gamma \gamma$ does occur at 1 loop level via intermediate virtual top quarks or W bosons (other electromagnetically charged particles contribute but are suppressed by smaller couplings): this channel played a central role in the discovery of the higgs and is a significant factor in Chapter 7.

Inverting the relationships in Eq. 2.77, we can rewrite the covariant derivative in terms of the post-electroweak symmetry breaking fields

$$
D_{\mu}=\partial_{\mu}-i \frac{g}{\sqrt{2}}\left(W_{\mu}^{+} T^{+}+W_{\mu}^{-} T^{-}\right)-i Z_{\mu}\left(g c_{W} T^{3}-g^{\prime} s_{W} Y\right)-i A_{\mu}\left(g s_{W} T^{3}+g^{\prime} c_{W} Y\right)-i g_{s} G_{\mu}^{b} \lambda_{b}
$$


with $T^{ \pm}$being the raising and lowering operators of $S U(2), T^{3}$ is the third generator of $S U(2)$, and $Y$ is the weak hypercharge. Utilizing two additional identities

$$
Q=T^{3}+Y \quad \text { and } \quad e=\frac{g g^{\prime}}{\sqrt{g^{2}+g^{\prime 2}}}
$$

on the photon term of Eq. 2.79 gives $-i e A_{\mu} Q$ - indicating that $Q$ represents electric charge and $e$ is the electromagnetic coupling from QED.

The time has come to return to the final piece of the SM Lagrangian, the Yukawa couplings from Eq. 2.68. Beginning with the quark sector, the relevant Lagrangian terms are

$$
\mathcal{L}_{\text {Yukawa }} \supset-y_{u} \bar{u}_{R} \tilde{H}^{\dagger} Q_{L}-y_{d} \bar{d}_{R} H^{\dagger} Q_{L}+\text { h.c. }
$$

for the first generation of quarks. For simplicity's sake, we explicitly define our fermion doublets as

$$
Q_{L}=\left(\begin{array}{c}
u_{L} \\
d_{L}
\end{array}\right) \quad \text { and } \quad L_{L}=\left(\begin{array}{c}
\nu_{L} \\
e_{L}
\end{array}\right)
$$

which, in the unitary gauge, leads to Lagrangian terms

$$
\mathcal{L}_{Y u k a w a} \supset-y_{u} \frac{v}{\sqrt{2}} \bar{u} u-\frac{y_{u}}{\sqrt{2}} h \bar{u} u .
$$

Thus, in a similar fashion to the gauge bosons, electroweak symmetry breaking has gifted mass and couplings to the up quark. However, things are a bit more complicated than they appear at first glance: there are three generations of quarks and including this leads to a more general form of the Lagrangian

$$
\mathcal{L}_{Y u k a w a} \supset-y_{u}^{i j} v \bar{u}_{L}^{i} u_{R}^{j}-y_{d}^{i j} v \bar{d}_{L}^{i} d_{R}^{j}-y_{e}^{i j} v \bar{e}_{L}^{i} e_{R}^{j}+\text { h.c. }
$$


where the Yukawa matrices are $3 \times 3$ complex matrices. This gives us terms of the form

$$
\begin{aligned}
\mathcal{L}_{Y \text { ukawa }} \supset-\frac{v}{\sqrt{2}}\left(\begin{array}{lll}
\bar{u}_{1} & \bar{u}_{2} & \bar{u}_{3}
\end{array}\right)_{R} \mathbf{Y}_{\mathbf{u}}\left(\begin{array}{c}
u_{1} \\
u_{2} \\
u_{3}
\end{array}\right)_{L}-\frac{v}{\sqrt{2}}\left(\begin{array}{lll}
\bar{d}_{1} & \bar{d}_{2} & \bar{d}_{3}
\end{array}\right)_{R} \mathbf{Y}_{\mathbf{d}}\left(\begin{array}{l}
d_{1} \\
d_{2} \\
d_{3}
\end{array}\right)_{L} \\
-\frac{v}{\sqrt{2}}\left(\begin{array}{lll}
\bar{e}_{1} & \bar{e}_{2} & \bar{e}_{3}
\end{array}\right)_{R} \mathbf{Y}_{\mathbf{e}}\left(\begin{array}{c}
e_{1} \\
e_{2} \\
e_{3}
\end{array}\right)_{L}+\text { h.c. }
\end{aligned}
$$

where the $\mathbf{Y}_{\mathbf{X}}$ terms are the Yukawa matrices. As a rather important aside, we note that due to the lack of a right-handed neutrino there are no neutrino mass terms generated. As a result, the neutrinos of the SM are massless.

In order to properly understand the fermion spectrum of the SM, we need to know the mass eigenstates of the SM as generically the Yukawa matrices do not have to be diagonal in the flavour basis. Explicitly diagonalizing the matrices using unitary matrices $U_{x}$ and $W_{x}$

$$
\mathbf{Y}_{\mathbf{u}}=U_{u} \mathbf{y}_{\mathbf{u}} W_{u}^{\dagger}, \quad \mathbf{Y}_{\mathbf{d}}=U_{d} \mathbf{y}_{\mathbf{d}} W_{d}^{\dagger}, \quad \mathbf{Y}_{\mathbf{e}}=U_{e} \mathbf{y}_{\mathbf{e}} W_{e}^{\dagger}
$$

gives diagonal matrices $\mathbf{y}_{\mathbf{x}}$ with positive elements. Applying the appropriate $U$ matrices to rotate the left-handed fields into the mass basis and the $W$ matrices to rotate the right-handed fields (e.g. $u_{L}^{i} \rightarrow U_{u}^{i j} u_{L}^{j}$ and $d_{R}^{i} \rightarrow W_{d}^{i j} d_{R}^{j}$ ) permits the cancellation of the unitary matrices in Eq. 2.86 for the lepton term - demonstrating that the weak and mass eigenstates are identical. Unfortunately, this isn't possible with the quark terms, as the transformation $Q_{L}^{i} \rightarrow W_{u}^{i j} Q_{L}^{j}$ can only cancel for either up or 
down quarks, but not both. In accordance with the standard convention, the up quarks are taken to have identical mass and weak eigenstates while the down quarks are left in their weak eigenstates.

Due to the down quarks no longer having mass eigenstates that match up with those of the up-type quarks, generation changing interactions are permitted through the charged-current interaction where we have quark bilinears of the form:

$$
\begin{aligned}
J_{L}^{+\mu} & =\left(\begin{array}{lll}
\bar{u}_{1} & \bar{u}_{2} & \bar{u}_{3}
\end{array}\right)_{L} \gamma^{\mu}\left(\begin{array}{l}
d_{1} \\
d_{2} \\
d_{3}
\end{array}\right)_{L}=\left(\begin{array}{lll}
\bar{u} & \bar{c} & \bar{t}
\end{array}\right)_{L} U_{u}^{\dagger} \gamma^{\mu} U_{d}\left(\begin{array}{l}
d \\
s \\
b
\end{array}\right)_{L} \\
& =\left(\begin{array}{lll}
\bar{u} & \bar{c} & \bar{t}
\end{array}\right)_{L} \gamma^{\mu} V\left(\begin{array}{l}
d \\
s \\
b
\end{array}\right)_{L} .
\end{aligned}
$$

$V \equiv U_{u}^{\dagger} U_{d}$ is the Cabibbo-Kobayashi-Maskawa (CKM) matrix [23, 24]. As a $3 \times 3$ unitary matrix, generically we would expect that it can be defined by three rotation angles and six complex phases, but this can be reduced to only one phase and three angles through chiral rotation of the quark fields. The final surviving phase leads to a direct violation of the combination of discrete symmetry operators $C$ (charge conjugation operator) and $P$ (parity operator, see Eq. 2.14), $C P$.

As a final point, I mentioned earlier that the neutrino is massless within the SM. Unfortunately, this prediction comes into direct conflict with reality as experimental results have shown that neutrinos undergo flavour oscillations and have non-zero, albeit tiny, masses. This indicates that the SM must be extended in some way. Further, the oscillations require the neutrinos to have their own analogue of the CKM matrix, which is referred to as the Pontecorvo-Maki-Nakagawa-Sakata (PMNS) matrix [25]. 


\subsection{Challenges to the SM}

The lack of neutrino masses is the first real issue that we have encountered so far this chapter that cannot be explained by the Standard Model and provides the first bit of hard evidence that, as a fundamental theory of nature, the SM is still incomplete. Mass terms can be added to the theory through two different mechanisms. The first involves providing the neutrino a standard Dirac mass through the introduction of a new $S U(2)$ singlet. This right-handed neutrino mixes with the SM left-handed neutrino, resulting in a very light mass eigenstate and a very heavy mass eigenstate. Alternatively, neutrinos could be their own antiparticles and form Majorana mass terms.

Although extremely interesting in their own right, massive neutrinos are just the beginning of the world beyond the Standard Model. As was hinted at in the introduction, the universe has provided many other phenomena that the SM has no explanation for: gravity, dark matter, dark energy, and the matter-antimatter asymmetry of the universe. In addition to the phenomena ignored by the SM, there is also a collection of theoretical problems that, while technically not incorrect or inconsistent, require extemporary justifications that lack strong theoretical underpinnings. These theoretical issues include the hierarchy problem, the strong CP problem, the number of SM parameters, and quantum triviality. All of these subjects have been studied in numerous BSM models, but, as of yet, no definitive experimental results that contradict the SM have been discovered.

Of all the aforementioned issues, the most important for our purposes is the hierarchy problem. Due to its incompleteness, we expect that the SM is actually a low-energy effective field theory (EFT) of some more fundamental high energy

theory $\mathcal{L}^{U V}$ which becomes relevant at some energy scale $\Lambda_{U V}$ [26]. For processes 
that occur well below $\Lambda_{U V}$ this isn't typically a problem as the physics in the UV can be integrated out and the EFT functions just fine for calculations. The Fermi four-fermion interaction is an excellent example of this in action: for low energies the theory works well, but when the energy of the interaction approaches the weak scale the full theory including the virtual $W$ boson needs to be taken into account. The SM as an EFT functions in a similar fashion, allowing us to package our ignorance in the form of higher mass dimension EFT operators. Since these operators are of mass dimension $>4$, they are non-renormalizable and only hold below the relevant mass scale as illustrated by the following generic dimension- 6 operator

$$
\mathcal{L}^{(6)} \supset \frac{C}{\Lambda_{U V}^{2}}(\bar{\psi} \psi)^{2}
$$

where $C$ is some constant factor. Everything to this point seems well and good, but there is trouble brewing in the higgs mass. The value for $-\mu^{2}=m_{h}^{2} / 2 \approx(88.4 \mathrm{GeV})^{2}$ was determined when the higgs was discovered in 2012 - however we know that the higgs mass receives gigantic quantum corrections from all the virtual particles that couple to it. In the SM, the most relevant fermion is the extremely heavy top quark due to its comparatively large coupling to the higgs. Explicitly, we take a typical Dirac fermion of mass $m_{f}$ that couples to the higgs through a Yukawa term $-\lambda_{f} \Phi \bar{f} f$. In this case, a 1-loop correction to the higgs mass parameter squared (the applicable Feynman diagram can be seen on the left side of Fig. 4.4) will give a contribution of the form

$$
\Delta m_{h}^{2} \propto-\frac{\left|\lambda_{f}\right|^{2}}{8 \pi^{2}} \Lambda_{U V}^{2}+\ldots
$$

for some UV cutoff scale $\Lambda_{U V}$.

We now have a problem! If $\Lambda_{U V}$ is at the Planck scale $\Lambda_{P l}$ then our corrections to 
the higgs mass squared will be about 30 orders of magnitude larger than the measured value. Furthermore, since all the massive particles in the SM get their mass from the higgs VEV - making the entire SM spectrum sensitive to these corrections.

Now, if new physics were to come in at energies not much larger than the electroweak scale these corrections wouldn't require huge tuning. The LHC, however, has turned up nothing of the sort at energy scales an order of magnitude or so above the higgs mass. Of course, there's nothing stating that the corrections cannot just miraculously cancel out to get the observed value for $m_{h}^{2}$ : perhaps there are an infinite or near-infinite number of universes and the only ones that can produce life capable of developing particle physics experiments require this type of precise cancellation. This line of thought is known as the anthropic principle and is a very real, albeit annoying, possibility. The search for a more satisfying answer to the hierarchy problem is a major motivating factor in many of the models explored within the rest of this work and will be discussed in more detail when BSM models are introduced in Chapter 4 


\section{Chapter 3}

\section{Cosmology}

\subsection{Introduction}

Perhaps the most stupefying part of our reality is the vastness of it all: trillions of galaxies each filled with hundreds of millions of stars populate a visible universe around 93 billion light-years in diameter. In this context, humanity remains stranded on a pale blue dot; even Voyager 1, the most distant man-made object, has yet to progress a single light-day from Earth (and it was launched in times so ancient they might as well be antiquity: 1977). Yet even more striking is the difference in scale between the proton $\sim 1.65 \times 10^{-15} \mathrm{~m}$ and the observable universe $\sim 8.8 \times 10^{26} \mathrm{~m}-$ more than 41 orders of magnitude! Similarly, the lifetime of a neutron is around 10 minutes whereas the universe is nearly 13.8 billion years old. However, these enormous gulfs don't mean that these worlds are disconnected. Take, for example, Big Bang Nucleosynthesis (BBN) when protons and neutrons in the early universe became bound into primordial nuclei (primarily Hydrogen and He-4, but also Deuterium, He3 , and Li-7) [27]: both the lifetime of the neutron and the relative energy of bound states are instrumental in determining the elemental composition of the universe, 
drastically affecting the formation of stars and galaxies. So, despite our focus being on the fundamental building blocks, we must turn our attention to the grandest of scales and look to see what the universe is trying to tell us should we be willing and able to listen.

Covering the entirety of cosmology (or even cosmology with direct ties to particle physics!) is a task that is so completely beyond any reasonable boundaries that it shall, perhaps unfortunately, not be attempted here. Instead, I focus on the areas that play a significant role in the phenomenology of the later chapters: the Cosmic Microwave Background (CMB), particles and relativistic degrees of freedom, and gravitational waves. Similar to Chapter 2, the content of this chapter is all well established physics. The primary sources used are Kolb and Turner's "The Early Universe" [27], James Hartle's "Gravity" [28], and Mark Trodden and Sean M. Carroll's "Introduction to Cosmology" [29].

\subsection{History of the Universe}

In short, the story of the universe is one of expansion, cooling, increasing entropy, and mystery. The final element is most strongly present early on: the first moments of our universe's infancy are a subject very heavy in speculation but very light in actual knowledge. The general mythology of pre-inflationary times begins with a Big Bang: spacetime as we know it is created from a primeval state (perhaps the decay of a false vacuum) and all the energy of the universe exists in a gravitational singularity at a single point. Cooling and expansion start immediately and, once the temperature drops below the Planck scale $1.22 \times 10^{19} \mathrm{GeV}$, current physics models begin to have some hope of functioning. Gravity decouples from the other forces and the universe is guessed to be in a hot plasma state where the non-gravitational forces remain 


\begin{tabular}{|c|c|c|c|}
\hline Event & Time & Temperature & Description \\
\hline Planck Epoch & $<10^{-43} \mathrm{~s}$ & $>10^{19} \mathrm{GeV}$ & $\begin{array}{l}\text { The Planck scale is the realm of } \\
\text { the unknown; quantum } \\
\text { corrections to general relativity } \\
\text { should render it invalid. }\end{array}$ \\
\hline $\begin{array}{c}\text { Grand } \\
\text { Unification }\end{array}$ & $\sim 10^{-36} \mathrm{~s}$ & $\sim 10^{16} \mathrm{GeV}$ & $\begin{array}{c}\text { The strong and electroweak forces } \\
\text { decouple, provided that nature is } \\
\text { described by a Grand Unified } \\
\text { Theory. }\end{array}$ \\
\hline Inflation & $\sim 10^{-32} \mathrm{~s}$ & $\begin{array}{c}10^{15}-10^{9} \\
\mathrm{GeV}\end{array}$ & $\begin{array}{c}\text { Cosmic inflation makes the } \\
\text { universe rapidly expand by a } \\
\text { factor of } \sim 10^{26} \text {. Reheating occurs } \\
\text { shortly after inflation ends. }\end{array}$ \\
\hline $\begin{array}{l}\text { Electroweak } \\
\text { Symmetry } \\
\text { Breaking }\end{array}$ & $\sim 10^{-12} \mathrm{~s}$ & $\sim 150 \mathrm{GeV}$ & $\begin{array}{l}\text { The higgs boson obtains a VEV, } \\
\text { creating the SM mass spectrum } \\
\text { we witness today in the } \\
\text { low-temperature limit. }\end{array}$ \\
\hline Hadronization & $\sim 10^{-5} \mathrm{~s}$ & $\sim 200 \mathrm{MeV}$ & $\begin{array}{l}\text { Quark-gluon plasma condenses } \\
\text { into hadrons as the QCD coupling } \\
\text { constant becomes strong and } \\
\text { chiral symmetry is broken. }\end{array}$ \\
\hline $\begin{array}{c}\text { Neutrino } \\
\text { Decoupling }\end{array}$ & $\sim 1 \mathrm{~s}$ & $\sim 1 \mathrm{MeV}$ & $\begin{array}{l}\text { The interaction rate between } \\
\text { neutrinos and the primordial } \\
\text { plasma drops below the expansion } \\
\text { rate causing neutrino decoupling }\end{array}$ \\
\hline $\begin{array}{c}\text { Big Bang } \\
\text { Nucleosynthesis }\end{array}$ & $10^{1}-10^{3} \mathrm{~s}$ & $100-1 \mathrm{keV}$ & $\begin{array}{l}\text { Protons and neutrons are bound } \\
\text { into primordial nuclei, resulting in } \\
\text { the population of the early } \\
\text { universe with Hydrogen, Helium, } \\
\text { and Lithium. }\end{array}$ \\
\hline Recombination & $\sim 370 \mathrm{kyr}$ & $\sim 0.4 \mathrm{eV}$ & $\begin{array}{l}\text { Electrons become bound to } \\
\text { nuclei, forming neutral atoms. } \\
\text { This transition allows photons to } \\
\text { free stream leading to the Cosmic } \\
\text { Microwave Background. }\end{array}$ \\
\hline Present & $\sim 13.8 \mathrm{Gyr}$ & $\sim 10^{-4} \mathrm{eV}$ & Life as we know it. \\
\hline
\end{tabular}

Table 3.1: History of the universe. 
unified, provided that nature is described by a Grand Unified Theory (GUT). Once the temperature of the universe drops below the GUT scale $\sim 10^{16} \mathrm{GeV}$, the strong force decouples from the electroweak force - giving us the behaviour we expect from the SM.

At this point, a period of inflation occurs [30]. Spacetime rapidly expands by a factor of at least $10^{26}$ during a period of supercooled expansion. While still somewhat speculative (the actual mechanism of inflation isn't known), this is the first part of the cosmic history that begins to have fairly strong justifications: inflation can solve two critical issues known as the horizon and flatness problems. In brief, the horizon problem is that the temperature of the Cosmic Microwave Background (CMB) radiation is remarkably uniform with relative fluctuations smaller than $10^{-4}$, indicating causal contact across the entire observable universe [30]; however when looking at the Hubble expansion of the universe only small sections (covering about $\sim 1 \%$ of the sky) should have been in causal contact. The flatness problem, on the other hand, arises from the fact that any departure of the density parameter from the critical value becomes rapidly larger as the universe evolves. Our universe has been measured as being very close to flat, meaning that the early universe would have an initial curvature radius tuned to one part in $10^{31}$ or less [30]. Inflation solves both these issues by putting the entire visible universe in causal contact early on and by the many-fold expansion flattening out the universe, automatically bringing us towards the very flat universe we see today. At the completion of inflation, a period of reheating takes place where the inflaton field decays into SM particles making the universe filled with a hot quark-gluon plasma.

With reheating behind us, we enter the world of more-or-less well-established and well-tested physics. Spontaneous symmetry breaking of $S U(2)_{L} \times U(1)_{Y}$ (as described in Sec. 2.7) occurred, granting the higgs a VEV, separating the electro- 
magnetic and weak forces, and giving mass to the SM fermions and gauge bosons. As the temperature of the universe continued to drop, the heavy particles of the SM (such as the higgs and the W bosons) began to "freeze out" or drop out of thermal equilibrium as their interaction rates became smaller than the expansion rate. A near eternity later (at $10^{-5} \mathrm{~s}$ after the Big Bang), the QCD phase transition started. Quarks and gluons became bound into hadrons (e.g. protons and neutrons) as the QCD coupling became strong and chiral symmetry was broken. Shortly thereafter, neutrinos, which were kept in thermal equilibrium via processes such as $\bar{\nu} \nu \leftrightarrow e^{+} e^{-}$ and $e \nu \leftrightarrow e \nu$, began to decouple from the plasma as the interaction rate dropped below the expansion rate of the universe. From here on, the neutrinos free stream and are unaffected by the thermal bath.

After the freeze out of the lightest SM particle remaining in thermal equilibrium, the electron, the thermal energy of the plasma was nearly fully transferred to the photon bath. This bath continued to cool until the photon energy was low enough to permit deuterium nuclei to survive. At this point, reaction chains producing Helium-4 were permitted and the primordial protons and neutrons become bound into primordial nuclei through a process known as Big Bang Nucleosynthesis (BBN). Measurements of the light element abundances in our universe match the predictions of BBN well, making it imperative that BSM models don't radically alter this process.

Following a wild first three minutes of existence, packed with universal phase transitions, expansions, and heavy particles, things quieted down: the universe was still in a plasma state of electrons, atomic nuclei, and photons and remained so for about 370,000 years. With the temperature of the universe then dropping to the sub$\mathrm{eV}$ range, electrons became bound to atomic nuclei causing the plasma to become electrically neutral. This resulted in the photon bath decoupling and the photons free streaming — these photons form the Cosmic Microwave Background (CMB) 
that permeate our universe today.

The remaining $\sim 13.8$ billion years that followed are of little importance to this work: stars, galaxies, supernovae, planets, and, sadly, biological life play no real role in the BSM models we examine (unless we take on some anthropic principle, but I will assiduously work against that until it becomes apparent that there is no other option). So, despite all the excitement in the billions and billions of years that follow the creation of the CMB, this time period will be viewed only as relevant insofar as the expansion of space redshifts propagating waves.

\subsection{Dynamics of the Universe}

As even the briefest of flings with general relativity reveals, the mathematics of curved spacetime can become incredibly nasty, incredibly quickly. Thankfully, the universe possesses symmetry at the grandest scales: it is both isotropic (the same in every direction) and homogeneous (the same at every point). This requirement greatly simplifies our computations as it forces the metric to be of the Friedmann-Lemaître-Robertson-Walker (FLRW) form

$$
d s^{2}=-d t^{2}+a(t)^{2}\left[d \rho^{2}+f^{2}(\rho)\left(d \theta^{2}+\sin ^{2} \theta d \phi^{2}\right)\right]
$$

where, in accordance with standard cosmology conventions and unlike the rest of this thesis, I use the mostly plus metric -+++ for the rest of this chapter. $a(t)$ is the scale factor that accounts for the expansion of the universe as a function of proper time $t$ for a comoving observer. Typically, we set $a$ (today) $=1$, making all prior times have $a<1$ and all future times $a>1$. This means that distances measured at some time $t_{1}$ will always be measured to be larger when measured at a later time $t_{2}$ (to wit

$\left.d\left(t_{2}\right)=d\left(t_{1}\right) \frac{a\left(t_{2}\right)}{a\left(t_{1}\right)}\right)$; as such, we often make use of comoving coordinates which factor 
out the scale factor — thus giving quantities that are independent of the scale of the universe.

A very important quantity know as the Hubble parameter is based on the scale factor

$$
H \equiv\left(\frac{\dot{a}}{a}\right)
$$

where $\dot{a}=\frac{d a}{d t}$. The Hubble parameter describes how quickly galaxies at distance $d$ move away from us due to the expansion of the universe

$$
v \sim H d
$$

This equation was expressed ${ }^{1}$ by Edwin Hubble in 1929 who calculated the current value for $H, H_{0}$, based on measurements of the velocities of distant galaxies [31]. The following (near) century of astronomy has both confirmed this relationship and improved the estimate of $H_{0}$.

Returning to Eq. 3.1, there are three options for $f(\rho)$ :

$$
\{\sin \rho, \rho, \sinh \rho\}
$$

which correspond to spherical, flat, and hyperbolic geometries respectively. Formally, the type of geometry described by this metric is local and can be embedded in larger, global manifolds. Eq. 3.1 can also be written in another useful form

$$
d s^{2}=-d t^{2}+a(t)^{2}\left[\frac{d r^{2}}{1-k r^{2}}+r^{2}\left(d \theta^{2}+\sin ^{2} \theta d \phi^{2}\right)\right]
$$

\footnotetext{
${ }^{1}$ Both Alexander Friedmann and Georges Lemaitre completed earlier work on the expansion of the universe, and the latter also suggested a proportionality between the velocity and distance of galactic bodies.
} 
with $k$ being 1 , 0 , or -1 for the spherical, flat, and hyperbolic scenarios.

Ultimately, we want to know how the scale factor evolves over time. In order to do this, Einstein's equation from GR

$$
R_{\mu \nu}-\frac{1}{2} R g_{\mu \nu}=8 \pi G T_{\mu \nu}
$$

must be used on the FLRW metric. From general relativity, $G$ is the gravitational coupling constant, $R_{\mu \nu}$ is the Ricci curvature tensor, $R$ is the scalar curvature, and $T_{\mu \nu}$ is the energy-momentum tensor. Eq. 3.6 demonstrates how local spacetime curvature interacts with the energy and momentum present within that spacetime. The perfect fluid form of the energy-momentum tensor is typically used for simplicity,

$$
T_{\mu \nu}=(\rho+p) U_{\mu} U_{\nu}+p g_{\mu \nu}
$$

for fluid four-velocity $U_{\mu}$, energy density $\rho$, and pressure $p$ (the latter two being taken in the fluid's rest frame). The fluid is comoving in the cosmological rest frame and thus the four-velocity can be taken to be $U^{\mu}=(1,0,0,0)$. It should be noted that the cosmological constant has not been explicitly included in Eq. 3.6. This has been done to simplify equations under the express recognition that including a cosmological constant is equivalent to including a energy-momentum tensor contribution of the form

$$
T_{\mu \nu}=\frac{-\Lambda}{8 \pi G} g_{\mu \nu}
$$

which is in the form of a perfect fluid with constant energy density - indicating that the cosmological constant is equivalent to vacuum energy.

Utilizing the FLRW metric (Eq. 3.5) and perfect fluid form of the energymomentum tensor (Eq. 3.7) in the Einstein equation (Eq. 3.6) allows us to derive two 
differential equations: the Friedmann equation

$$
H^{2}=\left(\frac{\dot{a}}{a}\right)=\frac{8 \pi G}{3} \sum_{i} \rho_{i}-\frac{k}{a^{2}}
$$

and the evolution equation

$$
\frac{\ddot{a}}{a}+\frac{1}{2}\left(\frac{\dot{a}}{a}\right)=-4 \pi G \sum_{i} p_{i}-\frac{k}{2 a^{2}} .
$$

The importance of the Friedmann equations grows from the fact that they link the rate of expansion (via the scale parameter) to the energy density.

The total energy density, $\rho$, is typically broken up into two terms representing the energy density from matter and radiation, respectively,

$$
\rho=\frac{\rho_{M}}{a^{3}}+\frac{\rho_{R}}{a^{4}}
$$

The scale factor dependence in the matter term corresponds to the fact that as the universe expands and the number of particles remains constant, the energy density will scale inverse to volume, $a^{-3}$. The radiation term undergoes the same volumetric scaling but also undergoes an additional process that provides another factor of $a^{-1}$ to the energy density: redshifting. The expansion of the universe leads to the wavelengths of photons becoming stretched out more and more over time, in proportion to the change in the scale factor

$$
\frac{\lambda_{o}}{\lambda_{e}}=\frac{a\left(t_{o}\right)}{a\left(t_{e}\right)} \equiv 1+z
$$

where the subscript $o$ indicates the quantity at the time of measurement, $e$ the quantity at the time of emittance, and $z$ the redshift. Since the energy of a photon is 
inversely related to its wavelength, this gives the proper $a^{-4}$ scaling for the radiation energy density. The energy density term associated with the cosmological constant term experiences no growth or loss - the universe's expansion involves the production of more vacuum and because this term arises from the vacuum nothing changes.

The difference in the evolution of these energy densities over time has led to three distinct eras of the universe in terms of the dominant type of energy density, each of which features different growth of the scale factor. In the case of a flat spacetime, we can use the Friedmann equation to show that the scale factor evolves in accordance with

$$
a(t)=a_{0}\left(\frac{t}{t_{0}}\right)^{2 / 3(1+w)}
$$

where $w$ is derived from the dependence of energy density on the scale factor:

$$
\rho(t) \propto \frac{1}{a(t)^{3(1+w)}}
$$

$w=0$ for matter and $w=1 / 3$ for radiation. In the case of the cosmological constant, the constant energy density leads to

$$
a(t) \propto e^{H t}
$$

Immediately after inflation the dominant form of energy is assumed to be radiation, causing the scale parameter to follow the scaling $a \propto t^{1 / 2}$. The harsher scaling of the energy density caused the radiation component of the energy density to become subdominant to the matter contributions $\sim 47,000$ years after the Big Bang. This era of matter domination continued for almost 10 billion years until matter was finally overtaken by the cosmological constant term and we entered the dark-energy era. Due to the energy density scaling, it is likely that dark energy will dominate the 
universe from here to eternity, ultimately resulting in the heat death of the universe.

Returning to the Friedmann equation Eq. 3.9, there is one more concept that we should note before pressing forth: critical density. If the universe features a density

$$
\rho_{c} \equiv \frac{3 H^{2}}{8 \pi G}
$$

then the local geometry must be flat. This leads to useful density notation

$$
\Omega \equiv \frac{\rho}{\rho_{c}}
$$

which indicates the local geometry: $\Omega>1$ indicates spherical geometry, $\Omega=1$ indicates flat geometry, and $\Omega<1$ indicates hyperbolic geometry. This notation is often broken up into contributions by different particles types $i$

$$
\Omega_{i}=\frac{\rho_{i}}{\rho} .
$$

Once again, our universe has been measured to be quite flat, so any BSM models that introduce new matter must ensure that any contributions they might make to $\Omega$ do not cause a significant deviation from $\rho_{c}$.

\subsection{Particles in the standard cosmology}

In broad strokes, we can classify the particles present throughout the cosmological history into two categories: in equilibrium and decoupled. The former case features particles that have an interaction rate that is $\gtrsim H$ which is the Hubble scale these interactions ensure that the particles are in thermal equilibrium with the rest of the plasma. Particles in the latter category are "frozen out", rarely interact with 
other particles, and undergo independent thermal evolution. A particle species with $g$ degrees of freedom (recalling that spin-0 particles have 1, massless higher spin particles have 2 , and massive higher spin particles have $2 s+1$ ) in thermal equilibrium at temperature $T$ and chemical potential $\mu$ obeys the following Bose-Einstein or FermiDirac distribution

$$
f(\mathbf{p})=\frac{1}{e^{\frac{E(\mathbf{p})-\mu}{T}} \pm 1}
$$

which describes bosons in the case of a minus sign and fermions in the case of a plus. This distribution can be easily utilized to obtain the number density, energy density, and pressure of the particle species

$$
\begin{aligned}
n_{i} & =\frac{g_{i}}{(2 \pi)^{3}} \int f_{i}(\mathbf{p}) d^{3} p, \\
\rho_{i} & =\frac{g_{i}}{(2 \pi)^{3}} \int E(\mathbf{p}) f_{i}(\mathbf{p}) d^{3} p \\
p_{i} & =\frac{g_{i}}{(2 \pi)^{3}} \int \frac{|\mathbf{p}|^{2}}{3 E(\mathbf{p})} f_{i}(\mathbf{p}) d^{3} p
\end{aligned}
$$

with $E(\mathbf{p})=\sqrt{m^{2}+\mathbf{p}^{2}}$ from special relativity. These integrals can be solved in both the high and low-temperature limits $(T \gg m$ and $T \ll m$, respectively); the results are outlined in Table 3.2. The results of integrating Eq. 3.20 suggest that it might be possible to find a compact expression for $\rho_{R}$ by adding up the contributions of each species. Not only would this be useful for checking if our understanding of SM particle physics matches up with the early universe, but hypothetical particles introduced in BSM models would also contribute - making this a useful cosmological constraint. Looking at Table 3.2, this appears simple; however, there is one wrinkle that must be dealt with: particles can be relativistic yet decoupled from the thermal bath. Furthermore, this isn't some rare occurrence that only happens in non-standard cosmologies. Neutrinos decouple before recombination and the creation of the CMB 
and do not receive the energy dumped into the photon plasma by electron-positron annihilation. To deal with this, we introduce the concept of effective temperature for a decoupled particle species

$$
T_{i}(a)=\left(\frac{a_{f}}{a}\right) T_{f}
$$

where $T_{f}$ and $a_{f}$ are the temperature and scale factor at the time of freeze out.

With Eq. 3.21 in hand, we can write down the energy density as a function of the relativistic degrees of freedom $g_{\star}$

$$
\rho_{R}=\frac{\pi^{2}}{30} g_{\star} T^{4}
$$

where

$$
g_{\star}=\sum_{\text {bosons }} g_{i}\left(\frac{T_{i}}{T}\right)^{4}+\frac{7}{8} \sum_{\text {fermions }} g_{i}\left(\frac{T_{i}}{T}\right)^{4}
$$

$T$ is the temperature of the background plasma (or photons after recombination) and the sums are over the relativistic bosons and fermions.

As the universe expanded and cooled, fewer and fewer particle species remained

\begin{tabular}{|c|c|c|c|}
\hline$n_{i}$ & $\begin{array}{c}\text { Relativistic } \\
\text { Bosons }\end{array}$ & $\begin{array}{c}\text { Relativistic } \\
\text { Fermions }\end{array}$ & $\begin{array}{c}\text { Low Temperature } \\
\text { Limit }\end{array}$ \\
\hline$\frac{\zeta(3)}{\pi^{2}} g_{i} T^{3}$ & $\left(\frac{3}{4}\right) \frac{\zeta(3)}{\pi^{2}} g_{i} T^{3}$ & $\left(\frac{m_{i} T}{2 \pi}\right)^{3 / 2} g_{i} e^{-m_{i} / T}$ \\
\hline$\rho_{i}$ & $\frac{\pi^{2}}{30} g_{i} T^{4}$ & $\left(\frac{7}{8}\right) \frac{\pi^{2}}{30} g_{i} T^{4}$ & $m_{i} n_{i}$ \\
\hline$p_{i}$ & $\frac{\rho_{i}}{3}$ & $\frac{\rho_{i}}{3}$ & $n_{i} T$ \\
\hline
\end{tabular}

Table 3.2: Key properties of particles in thermal equilibrium. $\zeta$ is the Riemann zeta function. 


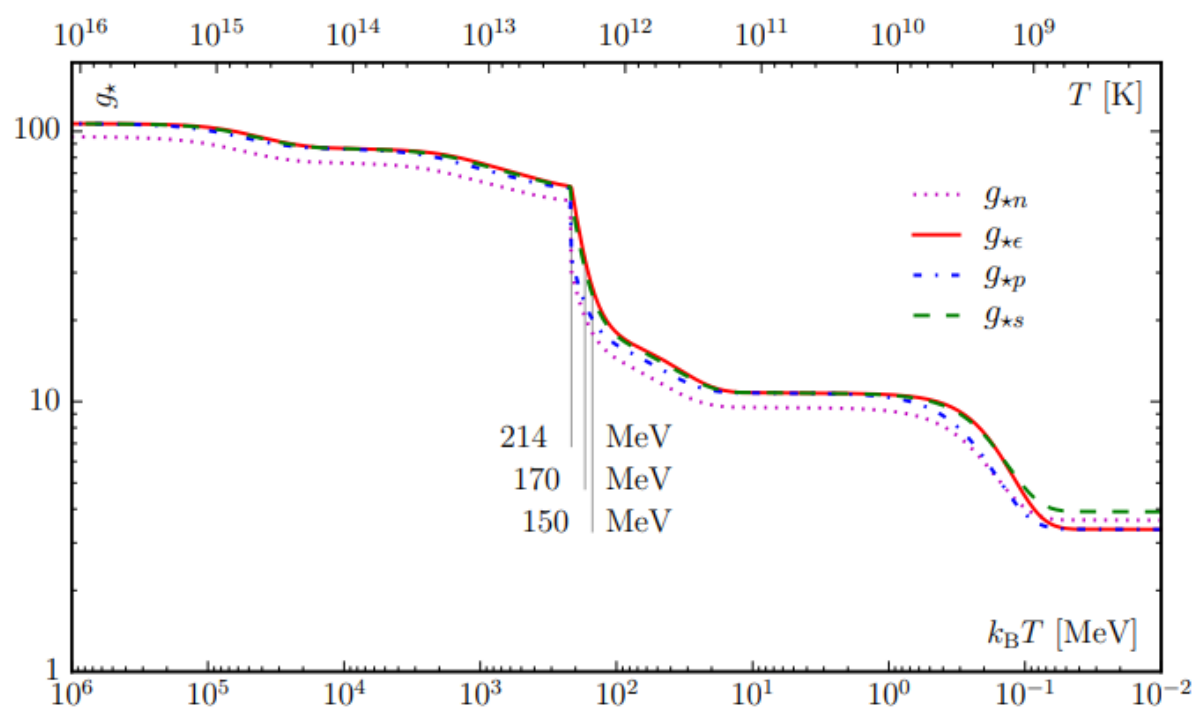

Figure 3.1: The evolution of effective relativistic degrees of freedom $\left(g_{\star \epsilon}\right)$ as a function of temperature. Also included are effective degrees of freedom for number density $\left(g_{\star n}\right)$, pressure $\left(g_{\star p}\right)$, and entropy $\left(g_{\star s}\right)$. From [32].

relativistic and in thermal equilibrium. This resulted in a progressive decrease in the effective degrees of freedom over time - this can be seen in Fig. 3.1. The most significant drop in $g_{\star}$ occurred during the QCD phase transition: 44.5 effective DOF were lost. The quarks and the gluons bound into pions, losing DOF for the quarks and gluons and gaining DOF for the pions. Explicitly, each quark flavour that was still in equilibrium prior to the phase transition (up, down, and strange) had $7 / 8$ $($ fermionic $) \times 2($ particle/antiparticle $) \times 3($ colour $) \times 2($ spin $)=10.5 \mathrm{DOF}$ lost gluons had 8 (colour) $\times 2($ spin $)=16$ DOF lost, and pions gained 3 DOF (baryons are not relevant to the DOF due to the lightest baryons having masses $\sim 1 \mathrm{GeV}$ ). Other transitions are fairly simple: particles either become too heavy to be produced and are simply removed from the calculation (e.g. the higgs boson) or the interaction rate drops below the expansion rate and the species freezes out (e.g. neutrinos) and the contribution must be calculated via Eq. 3.23. 
Another useful quantity that must be discussed is the rest-frame entropy density per comoving volume

$$
s=\frac{S}{a^{3}}=\frac{p+\rho-\sum_{i} \mu_{i} n_{i}}{T}
$$

with $\mu_{i}$ and $n_{i}$ being the chemical potential and comoving number density for species $i$. This indicates that the entropy density goes as $T^{-3}$ rather than $T^{-4}$ like energy density. As such, the scaling for the relativistic degrees of freedom for entropy is slightly different

$$
g_{\star s}=\sum_{\text {bosons }} g_{i}\left(\frac{T_{i}}{T}\right)^{3}+\frac{7}{8} \sum_{\text {fermions }} g_{i}\left(\frac{T_{i}}{T}\right)^{3}
$$

allowing us to write an expression for $s$

$$
s=\frac{2 \pi}{45} g_{\star s} T^{3}
$$

It should be noted that for relativistic particles in thermal equilibrium $g_{\star}=g_{\star s}$ and that differences only arise when a relativistic particle has decoupled from the thermal bath. In the case of the SM, this only occurs through neutrino decoupling and thus the effective degrees of freedom are identical throughout the early epochs of the universe. It should be noted that there are two other quantities, the effective degrees of freedom for number density $g_{\star n}$ and pressure $g_{\star p}$, that are not utilized in the papers presented here. In the case of $g_{\star n}$, each fermionic degree of freedom only contributes $3 / 4$ rather than $7 / 8$ due to the different constant factor seen in the relativistic limit.

One related and particularly useful cosmological observable is the number of effective neutrino species, $N_{\text {eff }}$. This quantifies the radiation energy density of the universe that does not come from photons

$$
\rho_{r}=\rho_{\gamma}\left(1+\frac{7}{8}\left(\frac{4}{11}\right)^{4 / 3} N_{\text {eff }}\right)
$$


where $\rho_{r}$ is the energy density of all radiation and $\rho_{\gamma}$ is the energy density of photons. The factor of $7 / 8$ is due to the neutrinos being fermions whereas the factor of $(4 / 11)^{4 / 3}$ comes from the temperature ratio between the photon bath and neutrinos; the photons have received additional energy from electron-positron annihilation that occurred post neutrino decoupling. In the SM, we would expect $N_{\text {eff }}$ to be 3 , however the actual value is slightly higher. This is due to the fact that neutrinos are not fully decoupled during electron-positron annihilation and also receive a (small) correction due to neutrino oscillations. With these factors taken into consideration, the SM value is $N_{\text {eff }}=3.045$ [33].

\subsection{Gravitational waves}

In 1916 Einstein predicted, as a consequence of general relativity, that massive bodies in non-spherical, non-uniform motion produce ripples in spacetime that propagated at the speed of light $[34,35]$. These so-called gravitational waves (GW) were a source of a good deal of skepticism (including from Einstein himself) in the early part of the 20th century with criticisms suggesting that solutions to the field equations would feature singularities. These singularities were later discovered to be coordinate singularities and, as such, were harmless to the theory. However, despite this leading to gravitational waves being accepted as a legitimate predication of general relativity, it wasn't until 2015, nearly a century after their prediction, that gravitational waves were first directly detected by the LIGO-Virgo collaborations. This huge delay between prediction and observation is due to the relative weakness of the gravitational interaction; despite being ubiquitous, gravitational waves only create a whisper of movement when they pass by ordinary matter. However, despite this attribute making gravitational waves incredibly difficult to detect, this same weakness of interaction means that grav- 
itational waves suffer little absorption and are able to transmit information from parts of spacetime that electromagnetic radiation cannot. More specifically, gravitational waves can provide a window to the unseen universe by serving as the fingerprints of binary mergers, collapse of black holes, and phase transitions of the early universe.

For the purposes of this thesis, the gravitational wave source of interest is vacuum phase transitions (PT). There are, broadly speaking, two types of phase transition: strong first order (or violent) and weak crossover. While PT of the latter type occur smoothly and don't produce detectable GW, strong first order phase transitions (SFOPT) lead to bubble nucleation as certain regions of the universe transition before others. These bubbles grow and eventually collide, creating huge amounts of turbulence and, crucially, gravitational waves. Intriguingly, these SFOPT, should they occur, take place across the entire sky, creating a stochastic gravitational wave background. This does not occur in the SM as both the electroweak and QCD PT are crossovers [36-39], but could be an important signature of many BSM models more on this in Chapters 4 \& 5 .

Because gravitational waves are very weak and spend most of their lifetimes propagating through mostly flat space, we can use the approximation of linearized gravitational waves where, instead of solving the full non-linear Einstein equation, we can use the linear approximation. Gravitational waves are transverse and can be treated as plane waves. Operating under the assumption that GW are a small perturbation on spacetime leads to the metric

$$
g_{\alpha \beta}(x)=\eta_{\alpha \beta}+h_{\alpha \beta}(x)
$$

where $\eta$ is the flat mostly minus metric. A plane gravitational wave propagating in 
the $z$-direction can be described by the perturbation

$$
h_{\alpha \beta}(t, z)=f(t-z)\left(\begin{array}{cccc}
0 & 0 & 0 & 0 \\
0 & 1 & 0 & 0 \\
0 & 0 & -1 & 0 \\
0 & 0 & 0 & 0
\end{array}\right)
$$

for any function $|f(t-z)| \ll 1$. This can be plugged into the metric Eq. 3.28 to give the line element

$$
d s^{2}=-d t^{2}+[1+f(t-z)] d x^{2}+[1-f(t-z)] d y^{2}+d z^{2}
$$

Voilà, we have a wave that is propagating in the $\mathrm{z}$ direction at the speed of light. The shape is dependent on the function $f(t-z)$ - for example a sinusoidal wave of amplitude $a$ and frequency $\omega$ could be described by $f(t-z)=a \sin [\omega(t-z)]$.

The perturbation described in Eq. 3.29 is not the most general form for a GW propagating along the $z$-axis as it only describes the first of two independent polarizations of a GW. The second is described by perturbations of the form

$$
h_{\alpha \beta}(t, z)=f(t-z)\left(\begin{array}{cccc}
0 & 0 & 0 & 0 \\
0 & 0 & 1 & 0 \\
0 & 1 & 0 & 0 \\
0 & 0 & 0 & 0
\end{array}\right) \text {. }
$$

This is typically referred to as the $\times$ (cross) polarization while perturbations of the form of Eq. 3.29 are called the + (plus) polarization. They can be combined to form a generalized expression for linearlized gravitational waves propagating parallel to the 
$z$-axis:

$$
h_{\alpha \beta}(t, z)=\left(\begin{array}{cccc}
0 & 0 & 0 & 0 \\
0 & f_{+}(t-z) & f_{\times}(t-z) & 0 \\
0 & f_{\times}(t-z) & -f_{+}(t-z) & 0 \\
0 & 0 & 0 & 0
\end{array}\right)
$$

where the plus and cross terms can feature different functions $f_{+}$and $f_{\times}$.

The effects of passing gravitational waves on matter can be seen by looking at the behaviour of two test masses. When a GW propagating in the $z$-direction that has an exclusively plus polarization passes by two test masses, one at the origin and the other at a distance $L_{\star}$ down the $x$-axis, the coordinates of the two points remain unchanged. However, the distance does change

$$
L(t)=\int_{0}^{L_{\star}}\left[1+h_{x x}(t, 0)\right]^{1 / 2} d x \approx L_{\star}\left(1+\frac{1}{2} h_{x x}(t, 0)\right)
$$

where the Taylor Series Expansion has been used in the second step due to the small size of the perturbation. This immediately gives

$$
\frac{\delta L(t)}{L_{\star}}=\left.\frac{1}{2} f(t-z)\right|_{z=0}
$$

The full generalization of this is fairly straight forward. For a test mass in the $z=0$ plane that is a distance $L_{\star}$ from the origin along some unit vector $\hat{\mathbf{n}}$ we can obtain the fractional strain produced by a gravitational wave

$$
\frac{\delta L(t)}{L_{\star}}=\frac{1}{2} h_{i j}(t, 0) n^{i} n^{j}
$$

The fractional strain produced by passing gravitational waves is the key signal that current gravitational wave detectors (such as LIGO) look for through the 
use of (essentially) large scale Michelson interferometers (see Fig. 3.2 for a simple schematic). The concept behind the experiments is fairly simple: a laser is divided by a beam splitter with the two separated beams being sent in different directions (typically perpendicular) towards mirrors. These mirrors reflect the beams back towards the splitter where they are recombined and sent into a detector. Depending on the relative length of the two arms of the detector varying degrees of constructive or destructive interference between the beams can take place - this can be measured by the detector. The point is that passing gravitational waves alter the lengths of arms, leading to changes in the interference pattern in accordance with the form of the perturbations of the metric associated with said GW. Of course, the actual implementation of this idea demands huge amounts of work, especially in the area of background mitigation. Similar to how the energy frontier in particle physics is dependent on building ever larger and more powerful colliders to probe ever higher energy scales, GW interferometers require longer and longer baselines to achieve sensitivities to even smaller strains: while the LIGO experiment had a baseline of $4 \mathrm{~km}$ [40], future experiments such as LISA involve satellites millions of kilometres apart [41]! With these new detectors comes the ability to probe a whole new world of BSM physics the possibility for looking for the phase transitions of hidden sectors is discussed in Chapter 5 .

\subsubsection{Detection of Stochastic Graviational Waves}

As a final bit of our introduction to gravitational waves, we must briefly touch on the framework that experiments use to determine their sensitivity. A stochastic gravitational wave background could be detectable if the signal-to-noise ratio (SNR) is above some threshold value, $\rho>\rho_{t h}$, dictated by the capabilities of future interferometers and pulsar timing arrays (PTAs). These interferometers or PTAs quote their 


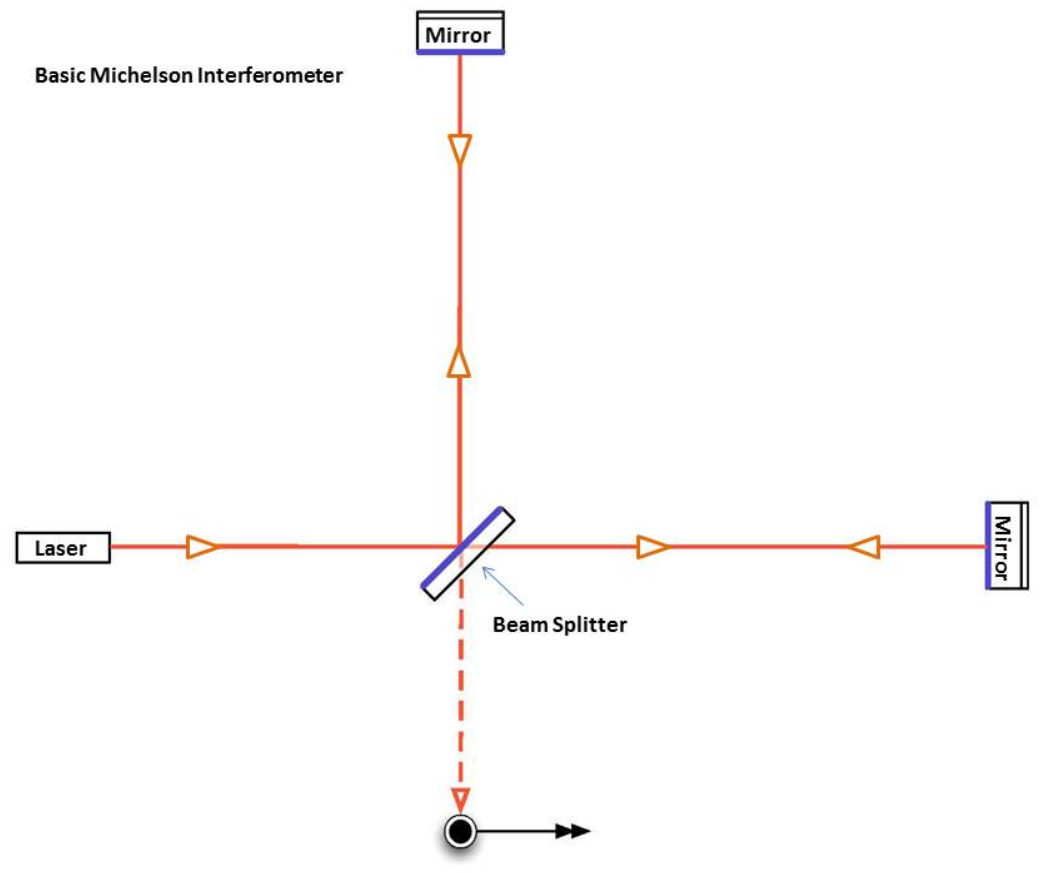

Figure 3.2: Schematic of a Michelson gravitational wave interferometer. The beam from the laser is divided by a beam splitter such that two separate beams travel along the two paths to the mirrors before being reflected back towards the beam splitter and being recombined and detected. [42]

experimental sensitivies in terms of spectral noise curves, $S_{\text {eff }}(f)$, which can be translated into units of energy density through $h^{2} \Omega_{\text {eff }}(f)=\frac{2 \pi^{2}}{3 H^{2}} f^{3} S_{\text {eff }}(f)$. If the experiment uses a single (multiple) detector, the autocorrelated (cross-correlated) SNR is used in comparing to the threshold value $\rho_{t h}$. The autocorrelated and cross-correlated SNR are explictly given as [43],

$$
\begin{gathered}
\rho^{2}=\mathcal{T} \int_{f_{\min }}^{f_{\max }} \mathrm{d} f\left(\frac{h^{2} \Omega_{\mathrm{GW}}(f)}{h^{2} \Omega_{\mathrm{eff}}(f)}\right)^{2} \quad \text { (autocorrelated) } \\
\rho^{2}=2 \mathcal{T} \int_{f_{\min }}^{f_{\max }} \mathrm{d} f\left(\frac{h^{2} \Omega_{\mathrm{GW}}(f)}{h^{2} \Omega_{\mathrm{eff}}(f)}\right)^{2} \quad \text { (cross-correlated) },
\end{gathered}
$$

where $\mathcal{T}$ is the exposure time of the experiment. The integration covers the entire broadband range of frequencies $\left(f_{\min }, f_{\max }\right)$. LISA [44] and B-DECIGO [17] are pro- 
posed to be single-detector interferometers, whereas BBO [45] and DEICIGO [46] would be built from an array of multiple interferometers. GW signals produced from an early cosmological phase transition would be seen as a stochastic background. Assuming that the GW follows a power law background in frequency, it is commonplace to quote the power law integrated (PLI) sensitivity curves [47]. The PLI curves are constructed using information from the power law form of the signal,

$$
h^{2} \Omega_{\mathrm{GW}}(f)=h^{2} \Omega_{\gamma}\left(\frac{f}{f_{\mathrm{ref}}}\right)^{\gamma}
$$

where $\gamma$ is the spectral index of the power law, and $f_{\text {ref }}$ is an arbitrary reference frequency which has no effect on the PLI sensitivities. $h^{2} \Omega_{\gamma}$ is the energy density calculated using Eq. (3.36) with spectral index $\gamma$ and reference frequency $f_{\text {ref }}$. The method of calculating the PLI curves involves plotting $h^{2} \Omega_{\mathrm{GW}}(f)$, using Eq. (3.37), for various spectral indices $\gamma$ and for some fixed threshold value of $\rho_{t h}$. Each curve will lay tangent to the PLI curve, more formally,

$$
h^{2} \Omega_{\mathrm{PLI}}=\max _{\gamma}\left[h^{2} \Omega_{\gamma}\left(\frac{f}{f_{\text {ref }}}\right)^{\gamma}\right] .
$$

The spectral noise curves used to create the PLI curves used in this work were taken from $[17,48-51]$ for the interferometers and [51,52] for the Square Kilometer Array (SKA) pulsar timing array. We have assumed an observation time of $\mathcal{T}=4$ years for the interferometers and $\mathcal{T}=5,10,20$ years for the various stages of SKA. In the case of the PTA experiments, the sensitivity curves are dependent on how frequently the pulsar's timing residuals, $\delta t$, are measured. When using Eq. (3.36) to construct the PLI curves for SKA, the upper integration bound is inversely proportional to pulsar's timing residual, $f_{\min }=1 / \delta t$. In this work, it is assumed that $\delta t=14$ days, 
but this may underestimate the capabilities of SKA as well as the cadences of the pulsar populations. If the timing residuals are lowered the maximum frequency reach of SKA increases, possibly giving the PTAs sensitivity to more scenarios. 


\section{Chapter 4}

\section{Beyond the Standard Model}

Up until this point, our discussion of the microscopic world has been laser focused on the safe haven that is the Standard Model - a resilient edifice that has survived wave upon wave of experimental evidence. Yet, in a decidedly imperfect world, nothing stands forever and even the most robust monuments must be refurbished, modified, or, on occasion, viciously torn down. Luckily (at least from the SM's perspective!), at the moment the success of the SM seems to indicate that the worst fate it might suffer is to fall into the role of a low energy effective field theory and, similar to Newtonian mechanics, provide a springboard to the next dominant paradigm. So, using the SM as our solid foundation, the time has come to start looking beyond the known and begin grappling with the many unsolved mysteries of our universe.

The incompleteness of the SM and the various theoretical issues like the hierarchy problem (outlined in Sec. 2.8) present the most obvious areas to challenge in our quest for a new, better theory and are the main motivations for the BSM models explored here. Of course, in the half-century that has followed the introduction of the SM, there have been numerous theories of new physics introduced: some thoughtfully constructed, strongly motivated, and thoroughly fleshed-out and a great 
many primarily inspired by the tantalizing thought of generating citations. Even if the discussion was limited to the former category, a proper exposé would take hundreds (if not thousands) of pages. As such, this background is limited to the models relevant to the phenomenology presented in this thesis: additional scalar multiplets, Nnaturalness, and supersymmetry (SUSY).

The work presented in this chapter, although still background material, is not established in a manner similar to the content of the previous chapters; none of the models have any experimental verification but instead present ideas to explain various phenomena with physics that evades current detection limits. Due to this, the primary sources for this chapter are reviews and papers rather than textbooks. Specifically, the references used for the hidden sector portal section are the collection of papers [53-56], while the Nnaturalness background comes from the original N. Arkani-Hamed et al. paper [57], and Stephen Martin's supersymmetry primer [10] is the basis for the section on supersymmetry.

\subsection{Hidden sector portals}

Our first foray into physics beyond the SM isn't a specific model; rather it is a mechanism that can be used in a huge variety of models that are trying to explain very different phenomena. The concept of a hidden sector (and its corresponding portal) is fairly straight forward: there is a collection of fundamental particles that do not interact with the particles of the SM through the strong, weak, or electromagnetic forces. The only connection (excluding gravity) between the SM and the hidden sector comes via some portal particle which can take a variety of forms. At first glance, a sector that is incredibly difficult to detect featuring particles and interactions that we can't see might seem like an uncalled-for fantasy, but this turns out to be a little too 
cynical and far too dismissive. Since the first evidence for dark matter (DM) in the form of galactic rotation curves was discovered by Rubin, Ford, and Freeman in the $1970 \mathrm{~s}^{1}[58,59]$, the case for matter that only interacts with regular, visible matter via gravity has grown increasingly more convincing as evidence from gravitational lensing, the CMB, structure formation, and galactic cluster collisions all point in the same direction. Recent estimates put DM as making up about $25 \%$ of the universe [7] — making a hidden sector particle description very tempting. Additionally, both the previously mentioned neutrino mass problem (see Sec. 2.8) and the baryon asymmetry problem (asking why all the structure we see in the universe is made up of matter and not antimatter) can be tackled through the introduction of hidden sectors, making them all the more compelling.

A general system for looking at BSM models, referred to as the portal framework, describes the interacting terms between hidden sector and SM particles as the product of operators

$$
\mathcal{L}_{\text {portal }}=\sum \mathcal{O}_{S M} \times \mathcal{O}_{H S}
$$

for operator $\mathcal{O}_{S M}$ built out of SM fields and operator $\mathcal{O}_{H S}$ built out of hidden sector fields. The sum consists of all possible combinations and dimensions. As usual, we care most about the lowest dimensional renormalisable terms that can be classified into four separate portal types: dark photon, dark higgs, axion, and sterile neutrino. These portals and their respective couplings are shown in Table 4.1.

Each of these portals is very interesting in its own right, however the research in the following chapters utilizes the scalar portal so we turn our attention to this particular case. In general, we can write down the Lagrangian for models containing

\footnotetext{
${ }^{1}$ Fritz Zwicky theorized dark matter in the 1930s based on observation of glaxy clusters, but this work was largely overlooked.
} 


\begin{tabular}{|c|c|}
\hline Portal Type & Coupling \\
\hline Dark photon $A_{\mu}$ & $\frac{-\epsilon}{\cos \theta_{W}} F_{\mu \nu}^{\prime} B^{\mu \nu}$ \\
\hline Dark higgs $S$ & $\left(\mu S+\lambda S^{2}\right) H^{\dagger} H$ \\
\hline Axion $a$ & $\frac{a}{f_{a}} F_{\mu \nu} \tilde{F}^{\mu \nu}, \frac{a}{f_{a}} G_{a \mu \nu} \tilde{G}_{a}^{\mu \nu}, \frac{\partial_{\mu} a}{f_{a}} \bar{\psi} \gamma^{\mu} \gamma^{5} \psi$ \\
\hline Sterile neutrino $N$ & $y_{N} L H N$ \\
\hline
\end{tabular}

Table 4.1: Types of portals and their respective lowest-order couplings. $F_{\mu \nu}^{\prime}, B_{\mu \nu}, G_{\mu \nu}, F_{\mu \nu}$ are the dark photon, hypercharge, gluon, and photon field strength tensors respectively. $S$ represents a new scalar singlet, $a$ a pseudoscalar axion, and $N$ a new neutral fermion.

a new singlet scalar $S$ in the form:

$$
\mathcal{L}=\mathcal{L}_{S M}+\mathcal{L}_{H S}+\left(\mu S+\lambda S^{2}\right) H^{\dagger} H
$$

The coupling $\mu$ has mass dimension 1 while $\lambda$ is dimensionless. All the purely hidden sector terms (including mass terms and self-interactions for the scalar singlet $S$ ) are contained in $\mathcal{L}_{H S}$. The $\mu S H^{\dagger} H$ term requires that $S$ is a full gauge singlet and leads to the mixing of the singlet state and the higgs boson $h$ after spontaneous symmetry breaking. When the mixing $\theta$ is small, it can be written as

$$
\theta=\frac{\mu v}{m_{h}^{2}-m_{S}^{2}}
$$

where $v$ is the higgs VEV. In models that feature this type of mixing, if $\lambda$ in Eq. 4.2 is taken to 0 then the entire parameter space of the new physics becomes purely 
dependent on just $m_{S}$ and $\theta$ making for a very simple, minimal extension to the SM: this type of model is examined in Chapter 6.

The $\lambda S^{2} H^{\dagger} H$ term is much more general than the trilinear term: the scalar singlet $S$ can have any quantum numbers and still form couplings of this type. This can be further extended to include generic scalar multiplets $X$ with multiple particles, $\lambda X^{\dagger} X H^{\dagger} H$. There are various directions that model builders can take this, including models that feature multiple scalar multiplets $X_{i}$. In these scenarios, it is also possible to create trilinear terms of the form $y\left(X_{i} H\right)^{\dagger} X_{j}$ provided that $X_{i}$ and $X_{j}$ have appropriate charges under $S U(2)_{L} \times U(1)_{Y}$. These terms create mass mixing among the multiplets and lead to a splitting of the mass spectrum. Scalar multiplets and their impacts on higgs decays are discussed in Chapter 7.

\subsection{Nnaturalness}

Nnaturalness is a framework developed by N. Arkani-Hamed et al. that utilizes multiple sectors ( $N$ to be precise!) to tackle the hierarchy problem. The actual details of all sectors are unimportant, save for the sector that contains the particles that make up the visible universe must be described by the SM. This makes $N$ naturalness an excellent framework to explore models with multiple hidden sectors and the possible phenomenological signals they could produce. The simplest version is "vanilla Nnaturalness" where every sector is a carbon copy of the SM with one small change:

the higgs mass parameters are allowed to range from $-\Lambda_{H}^{2}$ to $\Lambda_{H}^{2}$ where $\Lambda_{H}$ is the scale that cuts off quadratic divergences. If the distribution of the $m_{H}$ values is random then some sectors should be (accidentally) tuned at the $1 / N$ level such that: $\left|m_{H}^{2}\right|_{\min } \sim \Lambda_{H}^{2} / N$.

The sector with the smallest non-zero VEV is taken to be the visible SM sector. 
In order for small values of $m_{H}^{2}$ to be populated, it must be stipulated that sectors can take on a positive higgs mass parameter. This critically important feature of Nnatrualness leads to two fundamentally different sectors: "standard sectors" which are similar to our own just with a larger higgs VEV and correspondingly heavier mass spectrum, and "exotic sectors" that do not feature standard electroweak symmetry breaking. The higgs mass parameters of each sector are described by

$$
\left(m_{H}^{2}\right)_{i}=-\frac{\Lambda_{H}^{2}}{N}(2 i+r)
$$

where $r$ is a dial that one can use to tune the model (vanilla Nnaturalness does require some slight tuning in order to not be ruled out by cosmological constraints). Of course $i=0$ gives the sector with the smallest VEV and thus represents the SM. The model set-up is represented pictorially in Fig. 4.1.



Figure 4.1: Sketch of the Nnaturalness set-up. [57]

The number of sectors $N$ can be adjusted based on the needs of the model builder: the additional degrees of freedom reduce the scale that gravity becomes strongly coupled, $\Lambda_{G}$, as the renormalization of the gravitational constant suggests 
$\Lambda_{G}^{2} \sim \frac{M_{p l}^{2}}{N}$. Thus, models with $N \sim 10^{4}$ can be used for small hierarchies where $\Lambda_{H} \sim 10 \mathrm{TeV}$ whereas $N \sim 10^{16}$ solves the full hierarchy problem with $\Lambda_{H} \sim \Lambda_{G} \sim$ $10^{10} \mathrm{GeV}$. As for the sectors themselves, standard sectors $\left(m_{H}^{2}<0\right)$ feature standard electroweak symmetry breaking just like the SM. The difference among such sectors lies in the fact that as we go to sectors with larger and larger higgs mass parameter the sectors' VEVs increase as well $v_{i} \approx v_{S M} \sqrt{i}$. This in turn increases the masses of all the fermions and massive bosons proportional to $\sqrt{i}$. This scaling also affects the QCD confinement scale due to the increasing quark masses.

The exotic sectors $\left(m_{H}^{2}>0\right)$ feature much more radical differences from our SM: there is no VEV for the higgs and electroweak symmetry is only broken at very low scales, $\Lambda_{Q C D}$, due to the QCD condensate after the phase transition from free quarks to confinement has occurred. This results in the $W$ and $Z$ bosons obtaining masses roughly comparable to QCD resonances while the fundamental fermions have masses produced through four-fermion interactions after the higgs multiplet has been integrated out. Explicitly,

$$
m_{f} \sim y_{f} y_{t} \Lambda_{Q C D}^{3} /\left(m_{H}^{2}\right)_{i} \leq 100 \mathrm{eV}
$$

with $y_{f}$ being the Yukawa coupling for fermion $f$. Intriguingly, the extremely light nature of these quarks leads to very different behaviour of the QCD phase transition - but more on this later.

The addition of millions of new sectors (or more!) immediately runs into a very serious issue: $N$ sectors each featuring relativistic degrees of freedom gift huge contributions to the number of effective neutrinos $N_{\text {eff }} . N_{\text {eff }}$ has been measured to $\mathcal{O}(10 \%)$ precision and is in good agreement with the $\mathrm{SM}$ value of $N_{\text {eff }}^{S M}=3.046[7,33]$; radical departures from this prediction are clearly a no-go. To rectify this issue, 
a "reheaton" field is introduced. After inflation, the universe's energy is dumped primarily into the reheaton which couples universally to all sectors. Now, the reheaton can generically be a boson or fermion and both scenarios are examined in the original paper. For our purposes we narrow our focus to just the scalar reheaton $\phi$. In order to avoid the $N_{\text {eff }}$ constraints, the reheaton must dump most of its energy into sectors with small values of the higgs mass parameter. This can be accomplished through making the reheaton a gauge singlet that is both lighter than the naturalness cutoff $\Lambda_{H} / \sqrt{N}$ and the dominant coupling to every sector's higgs. The resulting Lagrangian terms are

$$
\mathcal{L}_{\phi}=-a \phi \sum_{i}\left|H_{i}\right|^{2}-\frac{1}{2} m_{\phi}^{2} \phi^{2}
$$

where the coupling $a$ has arbitrary sign for each sector and has scaling $\sim 1 / N$ to ensure that the loop induced mass for $\phi$ is not much larger than $\Lambda_{H} / \sqrt{N}$. Cross-quartic couplings of the form $\kappa\left|H_{i}\right|^{2}\left|H_{j}\right|^{2}$ are taken to be absent (their presence could potentially affect the spectrum and ruin the solution to the hierarchy problem), suppressed by small couplings.

The terms in Eq. 4.6 determine how the reheaton decays into particles for each sector. The Feynman diagrams for these processes are shown in Fig. 4.2; a quick glance clearly demonstrates that the decay processes for standard sectors are quite different than those leading to exotic sector particle production. Typically, the mass of the reheaton is taken to be around $100 \mathrm{GeV}$ so the lower diagrams are the relevant decays and can be described by effective Lagrangian terms

$$
\begin{aligned}
& \mathcal{L}_{\phi}^{v \neq 0} \supset C_{1} a y_{q} \frac{v}{m_{h}^{2}} \phi q q^{c} \\
& \mathcal{L}_{\phi}^{v \neq 0} \supset C_{2} \frac{g^{2}}{16 \pi^{2} m_{h}^{2}} \phi W_{\mu \nu} W^{\mu \nu}
\end{aligned}
$$



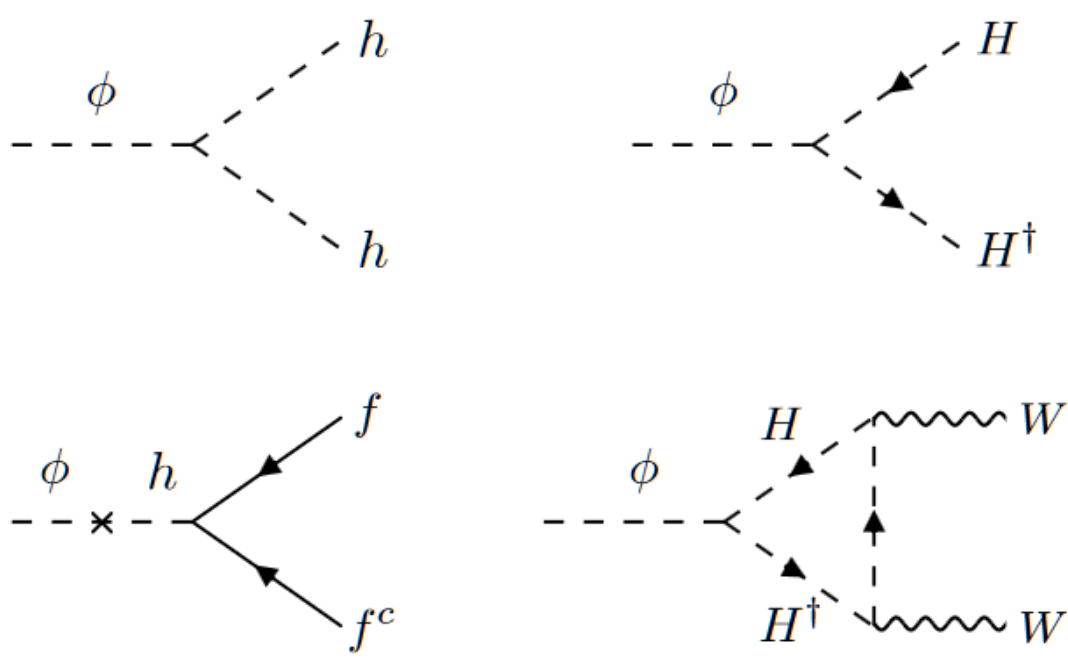

Figure 4.2: Decays for a scalar reheaton. The left (right) column is for the standard (exotic) sectors while the top (bottom) row is for $m_{\phi} \gg\left|m_{H}\right|\left(m_{\phi} \ll\left|m_{H}\right|\right)$. [57]

after the higgs particles have been integrated out. $C_{i}$ are numerical constants, $g$ is the weak coupling, and $W_{\mu \nu}$ is the $S U(2)$ field strength tensor. There is also a one loop decay $\phi \rightarrow \gamma \gamma$ for standard sectors, however this is always subdominant. The effective Lagrangian terms lead to decay widths that scale $\Gamma_{v \neq 0} \sim 1 / m_{h}^{2}$ and $\Gamma_{v=0} \sim 1 / m_{h}^{4}$ for the standard and exotic sectors, respectively. This is exactly the type of scaling we hoped for! The energy dumped into each sector falls off rapidly as the sector index $i$ grows, permitting the framework to skirt cosmological constraints.

In addition to the scaling of the decay widths inverse to the higgs mass parameter, the increasing mass of the fermions within the standard sectors $\left(m_{f} \sim v_{i} \sim\right.$ $v_{S M} \sqrt{i}$ ) results in decay channels of the reheaton becoming kinematically inaccessible once $m_{\phi}<2 m_{q}$, further reducing the amount of energy dumped into sectors with large higgs mass parameters. The energy densities of the various sectors of vanilla Nnaturalness are shown in Fig. 4.3.

Further discussion of Nnaturalness is left for Chapter 5 where the relevant cosmological constraints and interesting phenomenological signals are introduced. 


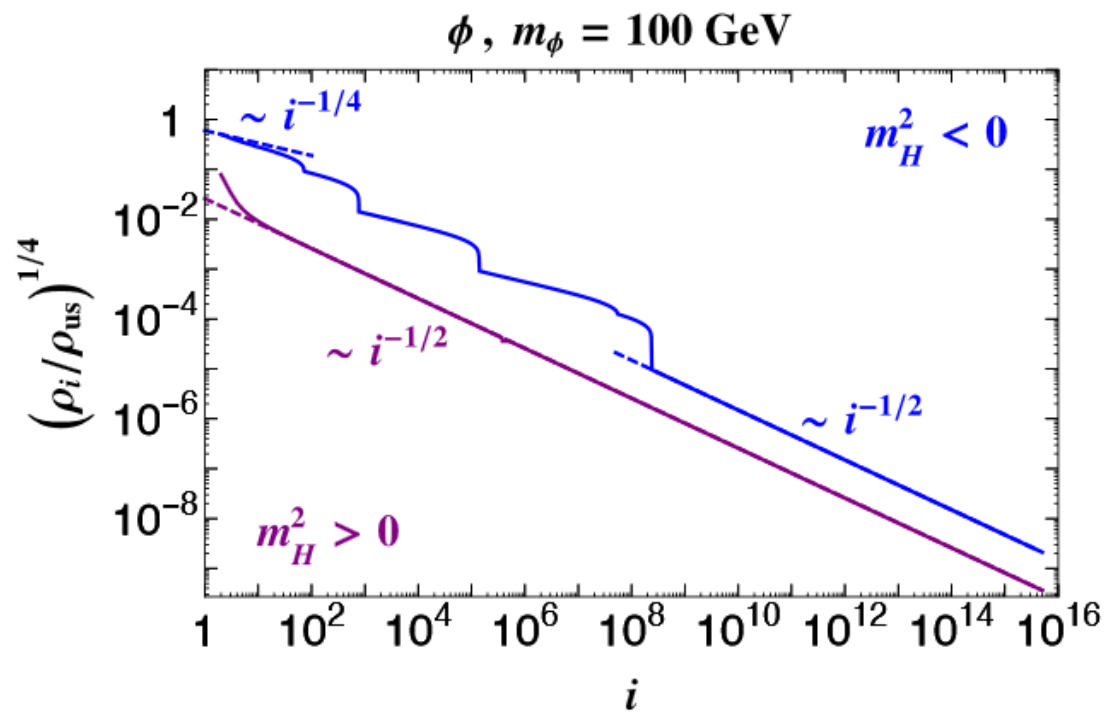

Figure 4.3: Energy densities as a function of sector index normalized to the energy of the SM sector for a $100 \mathrm{GeV}$ reheaton. The blue line represents the scaling for standard sectors while the purple line shows the energy density for exotic sectors. The drops in the standard sector energy density correspond to thresholds of the form $m_{\phi} \ll 2 m_{c_{i}}$ where a quark species becomes too heavy for the reheaton to decay to. [57]

\subsection{Supersymmetry}

\subsubsection{Overview}

Now, at long last, we turn our attention to the (much disputed) king of BSM models, supersymmetry (SUSY). Although there are many motivations for SUSY including dark matter candidates and the ability to construct grand unification (GUT) theories, the most compelling motivation comes from the hierarchy problem of the SM discussed in Sec. 2.8. As previously mentioned, if we take a typical Dirac fermion of mass $m_{f}$ that couples to the higgs through a Yukawa term $-\lambda_{f} \Phi \bar{f} f$ then a 1-loop correction to the higgs mass parameter squared will give a contribution of the form

$$
\Delta m_{h}^{2}=-\frac{\left|\lambda_{f}\right|^{2}}{8 \pi^{2}} \Lambda_{U V}^{2}+\ldots
$$


giving the higgs mass (and, indirectly, the rest of the SM mass spectrum) a sensitivity to the cutoff scale $\Lambda_{U V}$. Even worse, any new heavy particles that exist above the $\mathrm{TeV}$ scale will give virtual corrections to the higgs mass at the order of the new particles, so even taking the cutoff scale to be non-physical doesn't solve the hierarchy issue.

Now, take a complex singlet particle $S$ of mass $m_{S}$. This will couple to the higgs through the interaction term $-\lambda_{S}\left(H^{\dagger} H\right)\left(S^{\dagger} S\right)$ and result in a correction to the higgs mass

$$
\Delta m_{h}^{2}=\frac{\lambda_{S}}{16 \pi^{2}}\left[\Lambda_{U V}^{2}-2 m_{S}^{2} \ln \left(\Lambda_{U V} / m_{S}\right)+\ldots\right]
$$

Again, we have the high scale dependence! The Feynman diagrams of the 1-loop contributions to the higgs mass for both fermions and scalars are shown in Fig. 4.4. Despite demonstrating that heavy scalars also lead to huge mass corrections, there is
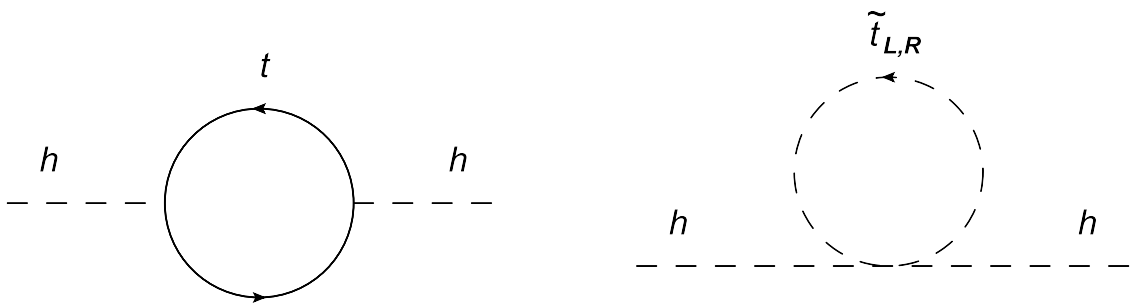

Figure 4.4: Contribution of the top and its partners to the Higgs mass. The quadratic divergences cancel. [60]

some hope to be had: if a fermion had two complex scalar partners with $\left|\lambda_{f}\right|^{2}=\lambda_{S}$ then the quadratic cutoff scale dependences in Eqs. 4.8 \& 4.9 cancel out. This leads to the fundamental idea of supersymmetry: a (super) symmetry relating fermions and bosons results in automatic cancellations of corrections to the higgs mass.

To this end, we introduce the fermionic operator $Q$ that takes fermions to bosons and vice-versa

$$
Q \mid \text { boson }\rangle=\mid \text { fermion }\rangle, \quad Q \mid \text { fermion }\rangle=\mid \text { boson }\rangle .
$$


Both $Q$ and its complex conjugate $Q^{\dagger}$ carry angular momentum and thus indicate that supersymmetry must be a spacetime symmetry. The Haag-Lopuszanski-Sohnius theorem requires that the supersymmetry generators satisfy (anti) commutation relations of the form [10]

$$
\left\{Q, Q^{\dagger}\right\}=P^{\mu}, \quad\{Q, Q\}=\left\{Q^{\dagger}, Q^{\dagger}\right\}=0, \quad\left[P^{\mu}, Q^{\dagger}\right]=\left[P^{\mu}, Q\right]=0
$$

with $P^{\mu}$ being the generator of spacetime translations.

Particles can be bundled into irreducible representations of the supersymmetry algebra that are referred to as supermultiplets. Supermultiplets contain both fermionic and bosonic states that are superpartners to each other - states that are proportional to each other up to factors of $Q, Q^{\dagger}$, and a spacetime translation or rotation. Since the operator $-P^{2}$ commutes with the supersymmetry generators all particles within the supermultiplet will have the same eigenvalues for this operator and thus the same mass. Finally, it can be shown through an application of the spin-statistics theorem that the number of bosonic degrees of freedom must be equal to the number of fermonic degrees of freedom for all supermultiplets, $n_{B}=n_{F}$.

Although there are many types of multiplets that can be constructed to obey the restrictions above, they are almost always ${ }^{2}$ reducible to two categories: chiral and gauge supermultiplets ${ }^{3}$. The former consist of a single Weyl fermion (with 2 helicity states) and two real scalar fields (typically assembled into a single complex scalar). Take, as an example, the right-handed electron supermultiplet $\bar{e}$ : it consists of complex spin-0 scalar, the right-selectron $\tilde{e}_{R}$, and the normal SM right-handed electron $e_{R}$. In general, the scalar superpartners to the SM fermions are referred to as "s-"

\footnotetext{
${ }^{2}$ This isn't the case for extended SUSY models that feature more than one copy of the generators $Q, Q^{\dagger}$, but these models play no role in the research presented here.

${ }^{3}$ There is also potentially a spin-2 graviton multiplet.
} 
particles: the quark superpartners are the squarks and the lepton superpartners are the sleptons. Especially important to the work in Chapter 7 are the top superpartners known as "stops" - more on that in the next subsection! The gauge supermultiplets, on the other hand, are combinations of a massless spin- 1 vector boson and its spin-1/2 Weyl fermion superpartner. In this case, the superpartner fermions are referred to with the "-ino" suffix: for example the $B^{0}$ superpartner is the bino $\tilde{B}^{0}$.

The field content of the version of SUSY utilized in Chapter 7, known as the Minimal Supersymmetric Standard Model (MSSM), is presented in Table 4.2. The only real surprise is that there are not one, but two higgs supermultiplets. This extra multiplet is required due to the fact that the fermionic higgsino is required to be an isodoublet with weak hypercharge of either $Y= \pm 1 / 2$. Regardless of the choice made, the new fermion spoils the gauge anomaly cancellation of the SM. In addition,

\begin{tabular}{|c|c|c|c|c|}
\hline & Spin-0 & Spin- $\frac{1}{2}$ & Spin-1 & $\begin{array}{c}\text { Gauge } \\
\text { charges }\end{array}$ \\
\hline$Q$ & $\left(\tilde{u}_{L}, \tilde{d}_{L}\right)$ & $\left(u_{L}, d_{L}\right)$ & - & $\left(\mathbf{3}, \mathbf{2}, \frac{1}{6}\right)$ \\
\hline $\bar{u}$ & $\tilde{u}_{R}^{\star}$ & $u_{R}^{\dagger}$ & - & $\left(\overline{\mathbf{3}}, \mathbf{1},-\frac{2}{3}\right)$ \\
\hline $\bar{d}$ & $\tilde{d}_{R}^{\star}$ & $d_{R}^{\dagger}$ & - & $\left(\overline{\mathbf{3}}, \mathbf{1}, \frac{1}{3}\right)$ \\
\hline$L$ & $\left(\tilde{\nu}_{L}, \tilde{e}_{L}\right)$ & $\left(\nu_{L}, e_{L}\right)$ & - & $\left(\mathbf{1}, \mathbf{2},-\frac{1}{2}\right)$ \\
\hline $\bar{e}$ & $\tilde{e}_{R}^{\star}$ & $e_{R}^{\dagger}$ & - & $(\overline{\mathbf{1}}, \mathbf{1}, 1)$ \\
\hline$H_{u}$ & $\left(H_{u}^{+}, H_{u}^{0}\right)$ & $\left(\tilde{H}_{u}^{+}, \tilde{H}_{u}^{0}\right)$ & - & $\left(\mathbf{1}, \mathbf{2}, \frac{1}{2}\right)$ \\
\hline$H_{d}$ & $\left(H_{d}^{0}, H_{d}^{-}\right)$ & $\left(\tilde{H}_{d}^{0}, \tilde{H}_{d}^{-}\right)$ & - & $\left(\mathbf{1}, \mathbf{2},-\frac{1}{2}\right)$ \\
\hline $\mathcal{G}$ & - & $\tilde{g}$ & $g$ & $(\mathbf{8}, \mathbf{1}, 0)$ \\
\hline $\mathcal{W}$ & - & $\tilde{W}^{ \pm} \tilde{W}^{0}$ & $W^{ \pm} W^{0}$ & $(\mathbf{1}, \mathbf{3}, 0)$ \\
\hline $\mathcal{B}$ & - & $\tilde{B}^{0}$ & $B^{0}$ & $(\mathbf{1}, \mathbf{1}, 0)$ \\
\hline
\end{tabular}

Table 4.2: Supermultiplets of the MSSM and their particle content. There are 3 generations of the quark and lepton supermultiplets. The first 7 entries are chiral supermultiplets, while the final three are gauge supermultiplets. The final column gives the supermultiplet representation under $S U(3)_{C} \times S U(2)_{L} \times U(1)_{Y}$ 
a higgs supermultiplet with $Y=1 / 2$ can only have Yukawa couplings with up-type quarks whereas a supermultiplet with $Y=-1 / 2$ can only have Yukawa couplings with down-type quarks (this is due to the requirement that the superpotential be holomorphic). To ameliorate these faults, a second higgs supermultiplet is added taking the opposite weak hypercharge of the first. This preserves the gauge anomaly cancellation of the SM and permits both types of Yukawas in one fell swoop.

Now, everything seems all well and good, however there is one critical issue remaining: according to the above construction we should have easily measured most of these superpartners decades ago. This is tragically not the case, indicating that we need to add another piece to the puzzle in the form of supersymmetry breaking — in other words, the vacuum state we exist in must violate supersymmetry. How exactly this occurs is, unsurprisingly, critically important; if the terms with dimensionless couplings break SUSY than the cancellation of the higgs mass corrections fails and our solution to the hierarchy problem is destroyed. Thus, the only way SUSY can be broken is through so-called soft symmetry breaking where the Lagrangian is split into two parts

$$
\mathcal{L}=\mathcal{L}_{S U S Y}+\mathcal{L}_{\text {soft }}
$$

the former term consists of all terms that preserve supersymmetry (all gauge and Yukawa interactions) whereas the latter contains mass terms and trilinear couplings with positive mass dimension — both types are permitted to break SUSY.

Ultimately, these soft breaking terms do lead to contributions to the higgs mass corrections

$$
\Delta m_{H}^{2} \propto \frac{m_{\text {soft }}^{2}}{16 \pi^{2}} \ln \left(\Lambda_{U V} / m_{\text {soft }}\right)+\ldots
$$

for $m_{\text {soft }}$ representing the scale of the largest soft terms. Amusingly, this has introduced a new (much smaller) hierarchy problem to the model: if the superpartner 
masses become too gigantic then the higgs mass parameter corrections once again become awkwardly large. This fact has led to much of the significant (and, sadly, often unjustified) criticism of SUSY: TeV scale superpartners are what basic SUSY naïvely suggests yet there has been no hint of such particles at the LHC.

\subsubsection{Stop Sector}

The world of SUSY is vast and often rather complicated. With the major pillars of the theory (briefly) outlined, it is time to turn our attention to the small sliver of SUSY that is relevant to the work done in Chapter 7, the stop sector.

We begin by outlining the three point couplings of the stop mass eigenstates, $\tilde{t}_{1}$ and $\tilde{t}_{2}$, to photons that arise from the Lagrangian terms,

$$
\mathcal{L} \supset-i e Q_{t}\left[\left(\tilde{t_{1}^{*}} \stackrel{\leftrightarrow}{\partial} \tilde{t_{1}}\right)+\left(\tilde{t_{2}^{*}} \stackrel{\leftrightarrow}{\partial} \tilde{t_{2}}\right)\right] A_{\mu}
$$

and the quartic terms from,

$$
\mathcal{L} \supset e^{2} Q_{t}^{2}\left(\tilde{t_{1}^{*}} \tilde{t_{1}}+\tilde{t_{2}^{*}} \tilde{t_{2}}\right) A_{\mu} A^{\mu}
$$

In both these cases, $e$ is the elementary charge and $Q_{t}$ is the electric charge of the stops. It should be noted that for higgs decays to diphotons, any vertex involving photons only couples to a single stop mass state. As a result, there are no "mixed" diagrams featuring both mass eigenstates in the loop and the only place the stop mixing angle (defined later in this section) is present is in the higgs-stop-stop coupling,

$$
\mathcal{L} \supset i\left[z_{11}\left(\tilde{t_{1}^{*}} \stackrel{\leftrightarrow}{\partial} \tilde{t_{1}}\right)-z_{12}\left(\tilde{t_{1}^{*}} \stackrel{\leftrightarrow}{\partial} \tilde{t_{2}}+\tilde{t_{2}^{*}} \stackrel{\leftrightarrow}{\partial} \tilde{t_{1}}\right)+z_{22}\left(\tilde{t_{2}^{*}} \stackrel{\leftrightarrow}{\partial} \tilde{t_{2}}\right)\right]
$$

Taking $c_{W}$ and $s_{W}$ to indicate the cosine and sine of the Weinberg angle, re- 
spectively, and $c_{t}$ and $s_{t}$ to represent the same for the stop mixing angle, we can explicitly express the Z- $\tilde{t}-\tilde{t}$ couplings arising from the above Lagrangian:

$$
\begin{aligned}
& z_{11}=-g_{Z}\left[T_{3 t} c_{t}^{2}-s_{W}^{2} Q_{t}\right]=-\frac{e}{2 s_{W} c_{W}}\left[c_{t}^{2}-\frac{4 s_{W}^{2}}{3}\right] \\
& z_{22}=-g_{Z}\left[T_{3 t} s_{t}^{2}-s_{W}^{2} Q_{t}\right]=-\frac{e}{2 s_{W} c_{W}}\left[s_{t}^{2}-\frac{4 s_{W}^{2}}{3}\right] \\
& z_{12}=g_{Z}\left[T_{3 t} c_{t} s_{t}\right]=\frac{e}{2 s_{W} c_{W}}\left[c_{t} s_{t}\right] .
\end{aligned}
$$

We also make use of the Z- $\gamma-\tilde{t}-\tilde{t}$ vertex which comes from the Lagrangian term (moving to the left-right basis of stops $\tilde{t}_{L}, \tilde{t}_{R}$ for compactness):

$$
\mathcal{L} \supset 2 e g_{Z}\left[Q_{t}\left(T_{3 t}-s_{W}^{2} Q_{t}\right) \tilde{t_{L}^{*}} \tilde{t_{L}}-s_{W}^{2} Q_{t}^{2} \tilde{t_{R}^{*}} \tilde{t_{R}}\right] Z_{\mu} A^{\mu}
$$

This leads us to our four-point $Z \gamma$ coupling terms

$$
\begin{aligned}
& z g_{11}=2 e g_{Z}\left[Q_{t} T_{3 t} c_{t}^{2}-s_{W}^{2} Q_{t}^{2}\right]=\frac{2 e^{2}}{3 s_{W} c_{W}}\left[c_{t}^{2}-\frac{4 s_{W}^{2}}{3}\right] \\
& z g_{22}=2 e g_{Z}\left[Q_{t} T_{3 t} s_{t}^{2}-s_{W}^{2} Q_{t}^{2}\right]=\frac{2 e^{2}}{3 s_{W} c_{W}}\left[s_{t}^{2}-\frac{4 s_{W}^{2}}{3}\right] \\
& z g_{12}=2 e g_{Z}\left[-c_{t} s_{t} Q_{t} T_{3 t}\right]=-\frac{2 e^{2}}{3 s_{W} c_{W}}\left[c_{t} s_{t}\right] .
\end{aligned}
$$

The higgs-stop coupling, $g_{h S S}$, is dependent on the parameters of the MSSM. As such, it is advantageous to express this coupling in a form that can be easily adjusted to account for different parameter configurations within the MSSM. In the left-right basis, the Lagrangian describing the higgs-squark-squark coupling is [61]:

$$
\begin{aligned}
\mathcal{L} \supset-\left[\frac{g m_{t}^{2} \cos \alpha}{m_{W} \sin \beta}\right. & \left.-g_{Z} m_{Z} \sin [\alpha+\beta]\left[\frac{1}{2}-\frac{2}{3} s_{W}^{2}\right]\right] \tilde{t}_{L}^{*} \tilde{t}_{L} h^{0} \\
& -\left(\frac{g m_{t}^{2} \cos \alpha}{m_{W} \sin \beta}-\frac{2}{3} g_{Z} m_{Z} \sin [\alpha+\beta] s_{W}^{2}\right) \tilde{t}_{R}^{*} \tilde{t}_{R} h^{0}
\end{aligned}
$$




$$
+\frac{g m_{t}}{2 m_{W} \sin \beta}\left(A_{t} \cos \alpha-\mu \sin \alpha\right)\left(\tilde{t}_{L}^{*} \tilde{t}_{R} h^{0}+\tilde{t}_{R}^{*} \tilde{t}_{L} h^{0}\right) .
$$

Here, $\alpha$ and $\beta$ are Higgs mixing angles, $A_{t}$ is a soft SUSY breaking term, and $\mu$ is the coefficient from the $\mu H_{u} H_{d}$ term in the Lagrangian. Explicitly, $\alpha$ and $\beta$ are the mixing angles that rotate between the gauge and mass basis of the higgs sector:

$$
\left[\begin{array}{l}
H_{u}^{0} \\
H_{d}^{0}
\end{array}\right]=\left[\begin{array}{l}
v_{u} \\
v_{d}
\end{array}\right]+\frac{1}{\sqrt{2}} R_{\alpha}\left[\begin{array}{l}
h^{0} \\
H^{0}
\end{array}\right]+\frac{i}{\sqrt{2}} R_{\beta}\left[\begin{array}{l}
G^{0} \\
A^{0}
\end{array}\right]
$$

for CP-even neutral scalars $H^{0}$ and $h^{0}$, CP-odd scalar $A^{0}$, Goldstone boson $G^{0}$ and where $R_{\alpha}$ and $R_{\beta}$ are the rotation matrices

$$
R_{\alpha}=\left[\begin{array}{cc}
\cos \alpha & \sin \alpha \\
-\sin \alpha & \cos \alpha
\end{array}\right], \quad R_{\beta}=\left[\begin{array}{cc}
\cos \beta & \sin \beta \\
-\sin \beta & \cos \beta
\end{array}\right]
$$

Although generically there are two Higgs doublets within the MSSM, experimental evidence points to both no new light scalars and $h_{0}$ behaving like a standard model Higgs [10]. As such, pushing up the mass of the other components of the scalar sector appears justified - ultimately adjusting the mixing angle alpha to a point where $h_{0}$ is decoupled from $H_{0}$ (the other, heavier CP-even neutral scalar in the MSSM), the decoupling limit. If working in the decoupling limit, where $\alpha \rightarrow \beta-\frac{\pi}{2}$, the Lagrangian can be simplified,

$$
\begin{aligned}
\mathcal{L} \supset-\left(\frac{g m_{t}^{2}}{m_{W}}+g_{Z} m_{Z}\right. & \left.\cos [2 \beta]\left(\frac{1}{2}-\frac{2}{3} s_{W}^{2}\right)\right) \tilde{t}_{L}^{*} \tilde{t}_{L} h^{0} \\
- & \left(\frac{g m_{t}^{2}}{m_{W}}+\frac{2}{3} g_{Z} m_{Z} \cos [2 \beta] s_{W}^{2}\right) \tilde{t}_{R}^{*} \tilde{t}_{R} h^{0}
\end{aligned}
$$




$$
+\frac{g m_{t}}{2 m_{W}}\left(A_{t}+\mu\right)\left(\tilde{t}_{L}^{*} \tilde{t}_{R} h^{0}+\tilde{t}_{R}^{*} \tilde{t}_{L} h^{0}\right)
$$

The relationship between the mass basis eigenstates and left-right basis eigenstates can be expressed in the following form with the rotation matrix,

$$
\left[\begin{array}{c}
\tilde{t}_{1} \\
\tilde{t}_{2}
\end{array}\right]=\left[\begin{array}{cc}
\cos \theta_{t} & \sin \theta_{t} \\
-\sin \theta_{t} & \cos \theta_{t}
\end{array}\right]\left[\begin{array}{c}
\tilde{t}_{L} \\
\tilde{t}_{R}
\end{array}\right] .
$$

The above rotation matrix is defined as the matrix that can diagonalize the stop mass matrix:

$$
\mathcal{M}_{\tilde{t}}=\left[\begin{array}{ll}
m_{L L}^{2} & m_{L R}^{2} \\
m_{L R}^{2} & m_{R R}^{2}
\end{array}\right] .
$$

We define the matrix using the same conventions as [62]:

$$
\begin{gathered}
m_{L L}^{2}=m_{Q_{3}}^{2}+y_{t}^{2} v_{u}^{2}+\tilde{\Delta}_{Q}\left(v_{d}^{2}-v_{u}^{2}\right), \\
m_{R R}^{2}=m_{U_{3}}^{2}+y_{t}^{2} v_{u}^{2}+\tilde{\Delta}_{U}\left(v_{d}^{2}-v_{u}^{2}\right), \\
m_{L R}^{2}=y_{t}\left(A_{t} v_{u}-\mu v_{d}\right) \equiv m_{t} X_{t},
\end{gathered}
$$

with $\tilde{\Delta}_{Q}=\frac{1}{2}\left(\frac{g^{2}}{2}-\frac{g^{\prime 2}}{6}\right), \tilde{\Delta}_{U}=\frac{g^{\prime 2}}{3}, \sqrt{v_{u}^{2}+v_{d}^{2}}=v=174 \mathrm{GeV}$, and $\tan \beta \equiv v_{u} / v_{d}$. When we diagonalize our stop mass matrix in Eq. (4.25), we arrive at eigenvalues $m_{1}$ and $m_{2}$ with $m_{2}>m_{1}$ by construction. The rotation of basis angle is defined by

$$
\cos \left[2 \theta_{t}\right]=\frac{m_{L L}^{2}-m_{R R}^{2}}{m_{2}^{2}-m_{1}^{2}} \text { and } \sin \left[2 \theta_{t}\right]=-\frac{2 m_{t} X_{t}}{m_{2}^{2}-m_{1}^{2}} .
$$

The previously expressed stop Lagrangian term in the left-right basis Eqs. (4.20 and 4.23) can now be rewritten in the stop mass basis: 


$$
\begin{array}{r}
\mathcal{L} \supset\left[\left(a c_{t}^{2}+b s_{t}^{2}+2 c_{t} s_{t}\right) \tilde{t}_{1}^{*} \tilde{t}_{1}+\left(a s_{t}^{2}+b c_{t}^{2}-2 c_{t} s_{t}\right) \tilde{t}_{2}^{*} \tilde{t}_{2}\right. \\
\left.+\left(-a c_{t} s_{t}+b c_{t} s_{t}+c\left(c_{t}^{2}-s_{t}^{2}\right)\right)\left(\tilde{t}_{1}^{*} \tilde{t}_{2}+\tilde{t}_{2}^{*} \tilde{t}_{1}\right)\right] h^{0}
\end{array}
$$

Here, $s_{t}=\sin \left[\theta_{t}\right], c_{t}=\cos \left[\theta_{t}\right]$, and $a, b$, and $c$ represent the coefficients on lines 1, 2, and 3 of the right-left basis Lagrangian (or the equivalent terms in the decoupling limit). Explicitly:

$$
\begin{aligned}
a & =-\frac{e m_{t}^{2}}{m_{W} s_{W}}-\frac{e m_{Z}}{c_{W} s_{W}} \cos [2 \beta]\left(\frac{1}{2}-\frac{2 s_{W}^{2}}{3}\right) \\
b & =-\frac{e m_{t}^{2}}{m_{W} s_{W}}-\frac{e m_{Z}}{c_{W} s_{W}} \cos [2 \beta] \frac{2 s_{W}^{2}}{3} \\
c & =\frac{e m_{t}}{2 m_{W} s_{W}}\left(A_{t}-\mu \cot \beta\right)
\end{aligned}
$$

in the decoupling limit. Ultimately, working in this limit leaves us with 4 dials that we can adjust within our study: $m_{1}, m_{2}, \theta_{t}$, and $\tan \beta$. 


\section{Chapter 5}

\section{Gravitational Wave Signals from}

\section{Multiple Hidden Sectors}

\section{$5.1 \quad$ Introduction}

The recent experimental detection of gravitational waves [63] gives humanity a new way to observe the universe. Future experiments $[17,44-46,52,64-68]$ will greatly expand the frequency range observable. Thus far, experiments have only observed recent events such as black hole mergers, but phase transitions in the early universe can leave an imprint as a stochastic gravitational wave background [69-74]. Thus, searches for this background of gravitational waves can give direct information of the history of the universe before big bang nucleosynthesis. Because gravity is universal, gravitational waves can allow us to probe hidden sectors that couple very weakly, or not at all, to the Standard Model as long they are reheated after inflation. This was first explored in [75], and there has been significant work on this idea since [51,76-92].

In this work, we explore the possibility of having multiple decoupled hidden

sectors. Large numbers of hidden sectors can solve the hierarchy problem as in the 
Dvali Redi model [93], in the more recently explored Nnaturalness [57] framework, or in orbifold Higgs models $[94,95]$. They can also be motivated by dark matter considerations [79,96-98]. Motivated by solutions to the hierarchy problem, we consider hidden sectors with the same particle content as the Standard Model that have all dimensionless couplings (defined at some high scale) equal to those of the Standard Model. The only parameter that varies across sectors is the dimension-two Higgs mass squared parameter, $m_{H}^{2}$. This simple ansatz can lead to very rich phenomenology and interesting gravitational wave spectra, but we stress that it is only a starting point for exploring the space of theories with multiple hidden sectors.

In this setup, there are two qualitatively different kinds of sectors:

- Standard Sectors: Those with $m_{H}^{2}<0$ where electroweak symmetry is broken by the vacuum expectation value (vev) of a fundamental scalar. As in [57], we assume that the standard sector with the smallest absolute value of $m_{H}^{2}$ is the Standard Model.

- Exotic Sectors: Those with $m_{H}^{2}>0$. In this case, electroweak symmetry is preserved below the mass of the Higgs, and broken by the confinement of QCD [99].

Cosmological observations, particularly limits on extra relativistic degrees of freedom at the time of Big Bang Nucleosynthesis and the time of the formation of the cosmic microwave background (CMB) [7], require that most of the energy in the universe is in the Standard Model sector as we will quantify. Therefore, the hidden sectors cannot be in thermal equilibrium at any time, and the physics of reheating must dump energy preferentially in the Standard Model sector. This can be accomplished with primordial axionlike particle (ALP) models $[100,101]$ and with the reheaton method [57]. We will also explore alternative parameterizations of reheating that 
satisfy this condition.

In all the above models, there is some energy in the hidden sectors, and these sectors undergo thermal evolution independent of the SM sector. If their initial reheating temperature is above their weak scale, the standard sectors will undergo phase transitions associated with the breaking of electroweak symmetry and with confinement of QCD. The exotic sectors will also undergo a phase transition when QCD confines and electroweak symmetry is broken simultaneously. The condition for these transitions to leave imprints on the stochastic gravitational wave spectrum is that they are strongly first-order phase transitions (SFOPT) [69-72]. This does not occur at either the electroweak or QCD phase transition in the SM, but as we will show, it does happen for the QCD phase transition in some standard sectors and in all exotic sectors that reheat above the QCD phase transition.

Ultimately, we demonstrate that while $N$ naturalness in its standard form will not lead to detectable gravitational wave signals, more general multi-hidden sector models can have SFOPT that generate detectable stochastic gravitational wave backgrounds. Further, we show that the spectrum of these signals can feature behaviour that diverges from the typically assumed power-law behaviour. Both runaway and non-runaway gravitational waves are considered over a range of inverse timescales.

This work is organized as follows: section 5.2 introduces the particle content of the model, section 5.3 discusses the phase transition behaviour of both the standard and exotic sectors present, section 5.4 lays out hidden sector reheating, section 5.5 applies constraints from cosmological observables allowing for the calculation of gravitational wave signatures in section 5.6, section 5.7 discusses non-runaway gravitational waves and, finally, section 5.8 ties everything up. 


\section{$5.2 \quad$ Particle Setup}

We consider the following Lagrangian as in [57]:

$$
\mathcal{L}=\sum_{i=-N / 2}^{N / 2} \mathcal{L}_{i}
$$

with $\mathcal{L}_{0}=\mathcal{L}_{\mathrm{SM}}$ being the Standard Model Lagrangian, and $\mathcal{L}_{i}$ being a copy of the SM Lagrangian with different fields, but with all dimensionless parameters the same. Each of the Lagrangians does contain a dimensionful operator:

$$
\mathcal{L}_{i} \subset-\left(m_{H}^{2}\right)_{i} H_{i}^{\dagger} H_{i}
$$

where $H_{i}$ is a Higgs field in each sector, and the mass term is parametrically given by

$$
\left(m_{H}^{2}\right)_{i} \sim-\frac{\Lambda_{H}^{2}}{N}(2 i+r)
$$

where $\Lambda$ is some high-scale cutoff, $N$ is the number of sectors, and $r$ is the mass parameter in the SM in units of $\Lambda_{H}^{2} / N$. We view the parameterization of Eq. (5.3) as a random distribution in theory space up to the cutoff $\Lambda$ : therefore, this setup solves the hierarchy problem if $r \sim \mathcal{O}(1)[57]^{1}$ and our sector is the one that that has the smallest absolute value of the Higgs mass parameter. We have taken for simplicity that there are equal numbers of sectors with positive and negative $m_{H}^{2}$, but this assumption does not affect our analysis. This Nnaturalness framework can be generalized: the various sectors can possess a wide range of particle content that can be freely selected by the model builder. The one exception to this is that "our" sector must consist of the Standard Model.

\footnotetext{
${ }^{1}$ Constraints require $r$ to be somewhat smaller than 1 .
} 
From the above Lagrangians, the Higgs in sectors with $i \geq 0$ will get a VEV given by

$$
v^{i}=\sqrt{-\left(m_{H}^{2}\right)_{i} / \lambda_{i}} \sim \Lambda_{H} \sqrt{\frac{2 i+r}{\lambda N}}
$$

where $\lambda_{i}$ is the quartic coefficient of the scalar potential and is the same across all sectors, $\lambda_{i}=\lambda$. This is another way to see how this framework can solve the hierarchy problem: the Higgs VEV is parametrically smaller than the cutoff for $N \gg 1$. The "standard sectors" with $i>0$ feature electroweak symmetry breaking just like in the $\mathrm{SM}$; however, the VEVs scale with the changing mass parameter: $v_{i} \sim v_{\mathrm{SM}} \sqrt{i}$. This means that the masses of the fermions and the $W$ and $Z$ will also increase proportional to $\sqrt{i}$. The consequences of this scaling on the confinement scale of QCD in the $i \geq 1$ sectors is further discussed in Sec. 5.3.

The "exotic sectors" with $i<0$ provide a radical departure from our own. $m_{H}^{2}>0$ leads to no VEV for the Higgs; electroweak symmetry is only broken at very low scales due to the phase transition from free quarks to confinement at the QCD scale $\Lambda_{Q C D}$ [99], and the masses of the $W$ and $Z$ are comparable to those of QCD resonances. The masses of fundamental fermions are produced via four-fermion interactions generated after integrating out the $\mathrm{SU}(2)$ Higgs multiplet. This leads to very light fermions:

$$
m_{f} \sim y_{f} y_{t} \Lambda_{Q C D}^{3} /\left(m_{H}^{2}\right)_{i} \leq 100 \mathrm{eV}
$$

with $y_{f}$ representing the Yukawa coupling to fermion $f$. As we will see, the extremely light quarks that appear in these sectors dramatically change the nature of the QCD phase transition - unlike the SM, the transition is strongly first order. Again, this is further developed in Sec. 5.3. Crucially, this results in the production of gravitational waves. This is the physical signature we explore in this paper; the calculation and results are presented in Sec. 5.6. 


\subsection{QCD Phase Transition}

We now study the nature of the QCD phase transition across the different sectors. Due to the confining nature of QCD, the exact nature of the phase transition is often difficult to ascertain analytically and requires the study of lattice simulations. In the SM, it is known that the phase transition is a crossover and does not lead to gravitational wave signals $[36,37]$. In the general case with three or more colours, the phase transition can be strongly first order in two regimes [102-104]:

- three or more light flavours and

- no light flavours.

Light indicates a mass small compared to the confinement scale $\Lambda_{Q C D}$, but what that means quantitatively is not precisely determined. In the SM, the up and down quarks are light, but the strange is not sufficiently light for an SFOPT. For the standard sectors in our setup, the quark masses increase with increasing VEV, so for sufficiently large $i$, all the quarks will be heavier than $\Lambda_{Q C D},{ }^{2}$ and those large $i$ sectors will undergo an SFOPT if they are reheated above the the confinement scale. Conversely, exotic sectors with zero VEV feature six very light quarks, so all the exotic sectors undergo SFOPT at the temperature of QCD confinement.

We now calculate the QCD confinement scale for each sector following the same procedure as [105]. First, due to the parameters of each sector being taken to be identical save for the Higgs mass squared (thus $v \neq v_{i}$, where $v$ is the SM VEV), we assume that the strong coupling of every sector is identical at some high scale.

\footnotetext{
${ }^{2} \Lambda_{Q C D}$ does vary with $i$, but the sensitivity is very weak as we will see below.
} 
Using the one-loop running, the $\beta$ function can be solved:

$$
\alpha_{s}^{i}(\mu)=\frac{2 \pi}{11-\frac{2 n_{f}^{i}}{3}} \frac{1}{\ln \mu / \Lambda^{i}},
$$

where $n_{f}^{i}$ is the number of quark flavours with mass less than $\mu / 2$ and $\Lambda^{i}$ is the scale where it would confine if all quarks remain massless. In the SM defined at scales well above all the quark masses, we have $\Lambda_{Q C D}=89 \pm 5 \mathrm{MeV}$ in $\overline{M S}$ [106]. Because we have set the strong couplings equal at high scales, $\Lambda=\Lambda^{i}$ for all $i$ at high scales for all sectors. However, since the masses of the quarks in each sector are different, we end up with a unique running of the coupling for each sector. At every quark mass threshold for a given sector, we match the coupling strengths above and below the threshold and determine the new $\Lambda^{i}$ for the lower scale. For example, at the mass of the top quark, we match a five-flavour coupling with the six-flavour one:

$$
\alpha_{s}^{i(5)}\left(2 m_{t}^{i}\right)=\alpha_{s}^{i(6)}\left(2 m_{t}^{i}\right)
$$

and thus

$$
\Lambda_{(5)}^{i}=\left(m_{t}^{i}\right)^{2 / 23}\left(\Lambda_{(6)}^{i}\right)^{21 / 23}
$$

Suppressing the $i$ 's for notational cleanliness, we can arrive at similar relations at the bottom and charm thresholds

$$
\begin{aligned}
& \Lambda_{(4)}=\left(m_{b}\right)^{2 / 25}\left(\Lambda_{(5)}\right)^{23 / 25}, \\
& \Lambda_{(3)}=\left(m_{c}\right)^{2 / 23}\left(\Lambda_{(4)}\right)^{25 / 27} .
\end{aligned}
$$

These can be combined to show that

$$
\Lambda_{(3)}=\left(m_{t} m_{b} m_{c}\right)^{2 / 27}\left(\Lambda_{(6)}\right)^{21 / 27}
$$


This type of matching procedure can be done as many times as necessary for a given sector. The process terminates when $\Lambda_{i}$ for a given scale is larger than the next quark mass threshold (i.e running the scale down arrives at the $\Lambda_{Q C D}$ phase transition before reaching the next quark mass scale). In cosmological terms, we can envision a sector's thermal history unfolding, whereas the plasma cools below each quark mass threshold and said quarks are frozen out. At a certain point, the sector arrives at the QCD phase transition and confinement occurs - if this occurs when $\geq 3$ quarks are at a much lower scale or all quarks have already frozen out, we get the desired phase transition.

\subsubsection{Standard Sectors}

As shown in Eq. (5.4), for standard sectors with increasing index $i$, the VEVs of said sectors increase $v_{i} \propto \sqrt{i}$. This leads to increasingly heavy particle spectra for higher sectors - eventually leading to sectors that are essentially pure Yang-Mills that feature strong first-order phase transitions. This, of course, prompts the question: at what index $i$ do said phase transitions begin? Using the methods outlined in the prior section we determine $\Lambda_{Q C D}$ to have a relevant value of

$$
\Lambda_{(2)}^{i}=\left(m_{s}^{i} m_{c}^{i} m_{b}^{i} m_{t}^{i}\right)^{2 / 29}\left(\Lambda_{(6)}^{i}\right)^{21 / 29}
$$

at the energy scale we're interested in. $\Lambda_{(6)}^{i}$ is identical for all sectors and is taken to have a Standard Model value of $\Lambda_{M S}^{(6)}=(89 \pm 6) \mathrm{MeV}$ [106]. Rewriting Eq. (5.11) in terms of Standard Model variables,

$$
\Lambda_{(2)}^{i}=\left(m_{s} m_{c} m_{b} m_{t} i^{2}\right)^{2 / 29}\left(\Lambda_{(6)}\right)^{21 / 29} .
$$


where $m_{q}$ without a superscript is the mass of $q$ in the SM. We take the sector with SFOPT to be the ones when the mass of the up quark, down quark, and QCD phase transition scale are all comparable:

$$
m_{u}^{i} \sim m_{u} \sqrt{i} \sim\left(m_{s} m_{c} m_{b} m_{t} i^{2}\right)^{2 / 29}\left(\Lambda_{(6)}\right)^{21 / 29}
$$

This can be solved for $i$ :

$$
i^{c} \sim \frac{\left(m_{s} m_{c} m_{b} m_{t}\right)^{4 / 21}\left(\Lambda_{(6)}\right)^{2}}{\left(m_{u}\right)^{58 / 21}} \sim 10^{6} .
$$

As we will see in Sec. 5.4, in the original Nnaturalness setup [57], the energy dumped into the $i$ th sector scales as $i^{-1}$, so there will not be enough energy in the sectors with $i>i^{c}$ to see a signature of these phase transitions. However, if we move away from the original $N$ naturalness reheating mechanism and begin exploring mirror sectors with large VEVs and with relative energy densities $\rho_{i} / \rho_{S M} \sim 10 \%$, a possibility allowed by current constraints, we can have sectors with relatively high dark QCD scales that produce detectable gravitational waves. From Eq. (5.11) we can determine the confinement scale of an arbitrary mirror sector. If we take Higgs VEVs as high as the GUT scale $\sim 10^{16} \mathrm{GeV}$, then we can use Eq. (5.12) to get confinement scales as high as $\sim 38 \mathrm{GeV}$. The signals of this sector and other test cases like it are explored in Sec. 5.6.

\subsubsection{Exotic Sectors}

In every exotic sector the fermion masses are exceptionally light: their masses are generated by dimension six operators with the Higgs integrated out as shown in Eq. (5.5), and are therefore all below the confinement scale. The exotic sectors 
all have identical one-loop running of the QCD gauge coupling, and thus all have approximately the same confinement scale given by $\Lambda_{\mathrm{ex}} \sim 90 \mathrm{MeV}$. These sectors all have six light fermions, so a strong first order phase transition occurs for all exotic sectors at this temperature. The confinement of these sectors directly leads to the production of both baryons and mesons as we have the spontaneous breaking of $\mathrm{SU}(6) \times \mathrm{SU}(6) \rightarrow \mathrm{SU}(6)$ and thus 35 pseudo-Goldstone bosons (pions). The masses obtained through the phase transition can be approximated through the use of a generalization of the Gell-Mann-Oakes-Renner relation [107,108],

$$
m_{\pi}^{2}=\frac{V^{3}}{F_{\pi}^{2}}\left(m_{u}+m_{d}\right)
$$

where $V \sim \Lambda_{Q C D}, F_{\pi}$ is the pion decay constant. One expects that within a given sector $F_{\pi} \sim V \sim \Lambda_{Q C D}[108]$ and as exotic sectors have $\Lambda_{e x} \sim 90 \mathrm{MeV}$ while the SM features $\Lambda_{Q C D}=(332 \pm 17) \mathrm{MeV}[106]$ we expect at most $\mathcal{O}(1)$ difference in the $\sqrt{\frac{V^{3}}{F_{\pi}^{2}}}$ coefficient relative to the SM value. So, for pions in exotic sector $i$ :

$$
m_{\pi}^{i} \sim \sqrt{\frac{m_{a}^{i}+m_{b}^{i}}{m_{u}+m_{d}}} m_{\pi}
$$

Here, $a$ and $b$ denote the component quark flavours.

\section{$5.4 \quad$ Reheating $N$ Sectors}

A key issue within Nnaturalness is how to predominantly gift energy density to our own sector so as to not be immediately excluded by cosmological constraints, particularly those from the effective number of neutrinos $\left(N_{\text {eff }}\right)$. Here we review the results of [57]. Reheating occurs through the introduction a "reheaton" field. After inflation, the reheaton field possesses the majority of the energy density of 
the Universe. Although this field can generically be either bosonic or fermionic, we reduce our scope to a scalar reheaton $\phi$. Our focus is primarily the production of gravitational waves from multiple sectors and a fermion reheaton does not change the scaling of the energy density of the exotic sectors and thus does not affect expected gravitational wave profiles.

In order to maintain the naturalness of our SM sector, the reheaton coupling is taken to be universal to every sector's Higgs. However, a large amount of the Universe's energy density must ultimately be deposited in our own sector for $N$ naturalness to avoid instant exclusion. In order to accomplish this, the decay width of the reheaton into each sector must drop as $\left|m_{H}\right|$ grows. If we insist that the reheaton is a gauge singlet that is both the dominant coupling to every sector's Higgs and lighter than the naturalness cutoff $\Lambda_{H} / \sqrt{N}$, then we construct a model that behaves as desired. The appropriate Lagrangian for a scalar reheaton $\phi$ is:

$$
\mathcal{L}_{\phi} \supset-a \phi \sum_{i}\left|H_{i}\right|^{2}-\frac{1}{2} m_{\phi}^{2} \phi^{2}
$$

Note that cross-quartic couplings of the form $\kappa\left|H_{i}\right|^{2}\left|H_{j}\right|^{2}$ that could potentially ruin the spectrum of $N$ naturalness are absent, taken to be suppressed by a very small coupling. Effective Lagrangians for the two different types of sectors present in this theory can be obtained by integrating out the Higgs bosons in every sector:

$$
\begin{aligned}
& \mathcal{L}_{\phi}^{v \neq 0} \supset C_{1} a y_{q} \frac{v}{m_{h}^{2}} \phi q q^{c}, \\
& \mathcal{L}_{\phi}^{v=0} \supset C_{2} a \frac{g^{2}}{16 \pi^{2} m_{H}^{2}} \phi W_{\mu \nu} W^{\mu \nu},
\end{aligned}
$$

with $C_{i}$ representing numerical coefficients, $g$ the weak coupling constant, and $W^{\mu \nu}$ the $\mathrm{SU}(2)$ field strength tensor. Immediately from Eq. (5.18), we can see that the matrix 
element for decays into standard sectors is inversely proportional to that sectors Higgs mass, $\mathcal{M}_{m_{H}^{2}<0} \sim 1 / m_{h_{i}}$ (since $v \sim m_{H}$ ). The loop decay of $\phi \rightarrow \gamma \gamma$ is always subleading and can be neglected. It should be noted that as one goes to sectors with larger and larger VEVs, the increasing mass of the fermions $\left(m_{f} \sim v_{i} \sim v_{S M} \sqrt{i}\right)$ eventually leads to situations where the decay to two on-shell bottom or charm quarks is kinematically forbidden, $m_{\phi}<2 m_{q}$. For sectors where this kinematic threshold is passed for charm quarks, the amount of energy in these sectors becomes so small that contributions to cosmological observables can be safely ignored. All in all, we end up with a decay width that scales as $\Gamma_{m_{H}^{2}<0} \sim 1 / m_{h}^{2}$. Since we can expect energy density to be proportional to the decay width, $\frac{\rho_{i}}{\rho_{S M}} \approx \frac{\Gamma_{i}}{\Gamma_{S M}}$, this indicates that energy density of standard sectors falls:

$$
\rho_{i} \sim r_{s} \frac{\rho_{S M}}{i}
$$

with $r_{s}$ being the ratio of the energy density of the first additional standard sector over the energy density of our sector. For the exotic sectors, Eq. (5.18) indicates a matrix element scaling $\mathcal{M}_{m_{H}^{2}>0} \sim 1 / m_{H_{i}}^{2}$ and is also loop suppressed. This leads to a significantly lower energy density than the standard sectors. Both the decay width and energy density for these sectors scale as

$$
\Gamma_{m_{H}^{2}>0} \sim \rho_{i} \sim 1 / m_{H}^{4} \sim 1 / i^{2}
$$

As a final note, in this setup the reheating temperature of the SM, $T_{R H}$, has an upper bound on the order of the weak scale. If this bound is not observed, the SM Higgs mass would have large thermal corrections - leading to the branching ratios into other sectors being problematically large [57]. Thus we only consider relatively low reheating temperatures $\lesssim 100 \mathrm{GeV}$. 
Ultimately, after examining the gravitational wave case produced by standard Nnaturalness, we also consider a more generic parameterization where the reheating temperature of each sector is a free parameter and is in general uncorrelated with the Higgs mass parameter. This allows us to explore a broader model space with multiple dark sectors at a huge range of scales. For these models, the reheating mechanism remains unspecified.

\subsection{Constraints}

In general, the multi-hidden-sector models explored feature a huge number of (nearly) massless degrees of freedom. Dark photons and dark neutrinos abound in these sectors and, assuming a relatively high reheat temperature, the leptons, quarks, and heavy bosons of these sectors can also be relativistic. In Nnaturalness this feature is realized quite dramatically: each of the $N$ sectors possesses relativistic degrees of freedom. The presence of these particles can have two main effects: extra relativistic particles can alter the expansion history of the universe through changes to the energy density or hidden sectors can feature annihilations that reheat the photons or neutrinos of our sector near Big Bang Nucleosynthesis (BBN) and affect the light element abundances. The effective number of neutrino species, $N_{e f f}$, is impacted by these contributions and, as such, is the strictest constraint that must be dealt with when studying these type of multi-phase-transition models. The SM predicts that $N_{e f f}^{S M}=3.046$ [109]. This is in good agreement with the $2 \sigma$ bounds from studies of the Cosmic Microwave Background (CMB) by Planck combined with baryon acoustic oscillations (BAO) measurements [7]:

$$
N_{\text {eff }}=2.99_{-0.33}^{+0.34}
$$


Various different assumptions about the history of the universe can be made and different data sets can be chosen to obtain slightly different results [51] — for the purposes of this exploratory work, wading through this landscape is unnecessary. Additionally,

$$
\frac{\left(\Delta N_{e f f}^{i}\right)_{C M B}}{\left(\Delta N_{e f f}^{i}\right)_{B B N}} \geq 1
$$

for any decoupled hidden sector [57]. Because the constraints on $N_{\text {eff }}$ are stronger at photon decoupling than at BBN, we can focus purely on the constraints provided by the former. Future CMB experiments [110] will improve the bound from Eq. (5.21) by about an order of magnitude. This could significantly reduce the allowed temperature ratio of any hidden sector or, alternatively, could provide evidence for such sectors in a way that is complementary to the gravitational wave signatures described below. For fully decoupled sectors that never enter (or reenter) thermal equilibrium with our sector, we obtain additional contributions to $N_{\text {eff }}^{S M}[51]$

$$
\Delta N_{\text {eff }}=\frac{4}{7}\left(\frac{11}{4}\right)^{4 / 3} g_{h} \xi_{h}^{4}
$$

Here, $g_{h}$ represents the effective number of relativistic degrees of freedom for the hidden sector ${ }^{3}$, and we parameterize the hidden sector temperature by [51]

$$
\xi_{h} \equiv \frac{T_{h}}{T_{\gamma}}
$$

and these should be evaluated at the time of photon decoupling. We take this approach and generalize it to include many additional sectors:

$$
\Delta N_{e f f}=\sum_{i} \frac{4}{7}\left(\frac{11}{4}\right)^{4 / 3} g_{i} \xi_{i}^{4} .
$$

\footnotetext{
${ }^{3} g_{h}=N_{\text {boson }}+7 N_{\text {fermion }} / 8$.
} 
For a dark sector with one relativistic degree of freedom, its temperature must be $T_{D S} \sim 0.6 T_{\mathrm{SM}}$ to not be excluded. Applying the energy density formula [111],

$$
\rho_{i}=\frac{\pi^{2}}{30} g_{i} T_{i}^{4}
$$

to both said dark sector and the SM and then taking the ratio indicates that the dark sector would have an energy density $\rho \sim 0.038 \rho_{\mathrm{SM}}$.

\subsubsection{Exotic Sector Contributions}

We begin by computing the constraints on exotic sectors; these are significantly weaker than those for standard sectors [57]. At the time of photon decoupling, $T_{\gamma} \sim 0.39 \mathrm{eV}$ while the temperature of the exotic sectors is lower. This means that for sectors with small and moderate $i$, we can use Eqs. (5.5) and (5.16) to see that the pions will be nonrelativistic leaving at most 7.25 effective degrees of freedom per sector from photons and neutrinos. For very large $i$, the pions can be much lighter, but those sectors also have very little energy in them in the standard reheating scenario. Coupling the number of effective degrees of freedom per sector with the energy density scaling of $\sim 1 / m_{H}^{4}$ as in Eq. (5.20) means that the zero VEV sectors have small temperature ratios. Assuming a reheating temperature of $100 \mathrm{GeV}$ and a completely uniform distribution of sectors, the temperature of the first exotic sector is slightly more than $6 \%$ of our sector at reheating. Applying Eq. (5.25) to this particular situation gives us:

$$
\Delta N_{e f f}=\sum_{i} \frac{4}{7}\left(\frac{11}{4}\right)^{4 / 3} g_{i}\left(\frac{\left(T_{R H_{E 1}} / T_{R H}\right)}{i^{1 / 2}}\right)^{4} \sim 10^{-4}
$$


with $T_{R H_{E 1}} / T_{R H}$ being the ratio of the reheat temperatures of the first exotic sector and our own sector (0.06 in standard Nnaturalness with $r=1)$. This sum is dominated by $i=1$; the sector with the lowest Higgs mass (and thus the most energy density) gives us a contribution of $\mathcal{O}\left(10^{-4}\right)$ to $\Delta N_{\text {eff }}$. Evolving the sector thermal histories forward in time to the recombination era gives us a slightly larger value, but still of order $\mathcal{O}\left(10^{-4}\right)$, well below current CMB bounds. It should be noted that modifying the exotic sectors' structure (e.g. adjusting the exotic sectors to have a lower Higgs mass squared or clustering multiple hidden sectors close to the first exotic one) leads to a $\Delta N_{\text {eff }}$ contribution that is larger than the base $N$ naturalness case. This increase is typically not excluded by current bounds, indicating a large degree of liberty in the structure and number of exotic hidden sectors.

\subsubsection{Standard Sector Contributions}

Within the context of vanilla Nnaturalness, the majority of contributions arise from standard sectors. This is explored in detail in [57]; here we briefly summarize these arguments. All additional standard sectors are very similar to our own: they have the same particle content and couplings and differ only by the Higgs mass. As our sector is taken to be the lightest so as to be preferentially reheated, every other standard sector features an earlier freeze-out of their respective particles. This ultimately leads to each sector having at most the same number of relativistic degrees of freedom as the SM.

In [57], the standard sector contributions are expressed as:

$$
\Delta N_{e f f}=\frac{1}{\rho_{\nu}^{u s}} \sum_{i \neq u s} \rho_{i} .
$$

In the case that the reheaton is lighter than the lightest Higgs (ours), this can be 
expressed as

$$
\begin{aligned}
\Delta N_{e f f} & \sim \sum_{i=1}^{N_{b}} \frac{1}{2 i+1}+\frac{y_{c}^{2}}{y_{b}^{2}} \sum_{i=N_{b}+1}^{N_{c}} \frac{1}{2 i+1} \\
& \simeq \frac{1}{2}\left(\log 2 N_{b}+\frac{y_{c}^{2}}{y_{b}^{2}} \log \frac{N_{c}}{N_{b}}\right)
\end{aligned}
$$

with $y_{c, b}$ representing the charm and bottom Yukawa couplings, respectively, and

$$
N_{b, c}=\left(\frac{m_{\phi}^{2}}{8 m_{b, c}^{2}}-\frac{1}{2}\right)
$$

with $m_{\phi}$ being the mass of the reheaton.

Application of these results indicates that for a majority of the parameter space, vanilla $N$ naturalness requires mild fine-tuning ( $r$ in Eq. (5.4) set to a value $\lesssim 1$ ). Numerical results for the fine-tuning required for various reheaton masses were presented in $[57]$.

\subsubsection{Generalized Reheating Scenarios}

The generalization of possible reheating mechanisms mentioned in section 5.4 where the reheating mechanism no longer depends on the Higgs' mass parameter of a given sector - opens up a wide range of hidden sectors for study. Specifically, this allows mirror sectors with large Higgs VEVs to be reheated to significant energy densities and thus produce gravitational waves with enough power to be detected. Crucially, despite this analysis being limited to mirror sectors with large Higgs masses, this analysis pertains to any strong, confining phase transition at high scales. Since $N_{\text {eff }}$ constraints remain our strongest cosmological bounds for massive standard sectors, our starting point for exploring the limits of high transition temperatures is Eq. (5.25). Assuming heavy, standard sectors (with the only relativistic 
particles being photons and neutrinos) we can saturate the bounds of Eq. (5.21) and solve for the maximum temperature allowed for any number of sectors:

$$
\begin{aligned}
& T_{i} \sim 0.38 T_{S M} \quad 1 \text { hidden sector }, \\
& T_{i} \sim 0.25 T_{S M} \quad 5 \text { hidden sectors, } \\
& T_{i} \sim 0.21 T_{S M} \quad 10 \text { hidden sectors, } \\
& T_{i} \sim 0.12 T_{S M} \quad 100 \text { hidden sectors, }
\end{aligned}
$$

where all the hidden sectors have the same temperature as one another.

Using these restrictions, we can examine the behaviour of standard sectors with a much larger VEV than our own. In terms of the $N$ naturalness framework, this means we can get an SFOPT for QCD if we look at sectors with $i$ greater than the critical index of Eq. (5.14) where all the quark masses are above the QCD confinement scale, as long as their temperatures are below the bounds presented here.

\subsection{Gravitational Wave Signals}

We now turn to the gravitational wave signatures of our setup. At high temperatures, each of the hidden sectors has QCD in the quark/gluon phase, but at temperatures around $\Lambda_{\mathrm{QCD}, \mathrm{i}}$, the $i^{\text {th }}$ sector undergoes a phase transition into the hadronic phase that we computed for the different sectors in Sec. 5.3. As discussed in that section, this phase transition will be strongly first order (SFOPT) for certain numbers of light quarks, which will generate gravitational waves. This differs from QCD in the SM sector, as the PT is a crossover and not first order [112]. A SFOPT proceeds through bubble nucleation, where bubbles of the hadronic phase form in the vacuum of the quark phase. These bubbles will expand, eventually colliding and merging until the

entire sector is within the new phase. These bubbles are described by the following 
Euclidean action [113]:

$$
S_{E}(T)=\frac{1}{T} \int d^{3} x\left[\frac{1}{2}(\nabla \phi)^{2}+V(\phi, T)\right],
$$

where the time component has been integrated out due to nucleation occurring not in vacuum but in a finite temperature plasma. $\phi$ is the symmetry-breaking scalar field with a nonzero VEV. In the case of the chiral phase transition, the scalar field breaking the $\mathrm{SU}\left(N_{f}\right)_{R} \times \mathrm{SU}\left(N_{f}\right)_{L}$ chiral symmetry is the effective quark condensate $\phi_{i} \sim\langle q \bar{q}\rangle_{i}$ of the respective sector. We leave the thermalized potential $V(\phi, T)$ general. As previously stated, an exact QCD potential at the time of the chiral phase transition is not well understood outside of lattice results. In [88] a chiral effective Lagrangian was used to calculate a low-energy thermalized potential for confining $\mathrm{SU}(N)$. The amount of energy density dumped into the individual sectors dictates the energy budget for the PT and hence for the gravitational waves. Assuming that the SM sector is radiation dominated, a quantity that characterizes the strength of the $\mathrm{PT}$ is the ratio of the latent heat of the phase transition, $\epsilon$, to the energy density of radiation, at the time of nucleation [114],

$$
\alpha \equiv \frac{\epsilon}{g_{*} \pi^{2}\left(T_{\gamma}^{n u c}\right)^{4} / 30},
$$

with $\epsilon$ being calculable from the scalar potential. Assuming that there is a negligible amount of energy being dumped back into the SM, which would cause significant reheating of $\rho_{\gamma}$, the latent heat $\epsilon$ should correspond to the energy density of the hidden sector going through the PT. The parameter $g_{*}$ in the denominator of Eq. (5.33) is the number of relativistic degrees of freedom at the time of the phase transition, with contributions from species in both the visible and dark sectors. It has weak 
temperature dependence in a single sector, but when dealing with multiple hidden sectors, $g_{*}$ gains contributions from all $N$ sectors' relativistic degrees of freedom, weighted by their respective energy densities

$$
g_{*}=g_{*, \gamma}+\sum_{i} g_{*, i}\left(\xi_{i}\right)^{4}
$$

with $\xi$ being the temperature ratio defined in Eq. (5.24). The bounds from effective number of neutrinos [7] mean that $\xi_{i} \lesssim 1$ for all $i$, so $g_{*} \approx g_{*, \gamma}$. In the case of dark QCD-like chiral phase transitions, the temperature of the phase transition is on the order of the symmetry-breaking scale of the respective sector, $T_{h}^{i} \sim \mathcal{O}\left(\Lambda_{Q C D, i}\right)$. The work of [88] calculated $\alpha$ with an effective chiral Lagrangian and found various upper bounds. We take the optimistic scenario where the numerator is bounded from above by the symmetry breaking scale

$$
\alpha_{i} \approx \xi_{i}^{4} \approx\left(\frac{\Lambda_{Q C D, i}}{T_{\gamma}^{n u c}}\right)^{4}
$$

where $T_{\gamma}^{\text {nuc }}$ is the temperature of the SM photon bath at the time of the phase transition. Another important parameter to characterize the phase transition is its inverse timescale $\beta$ [73]. The inverse timescale can be calculated using the action in Eq. (5.32):

$$
\left.\beta \equiv \frac{d S_{E}(T)}{d t}\right|_{t=t_{n u c}} .
$$

The ratio of $\beta$ and the Hubble constant, at the time of nucleation, $H$ controls the strength of the gravitational wave (GW) signal,

$$
\frac{\beta}{H}=\left.T_{h}^{n u c} \frac{d S_{E}(T)}{d T}\right|_{T=T_{h}^{n u c}}
$$


Due to the lack of a general analytic QCD potential, it is not possible to use Eq. (5.37) to calculate $\beta / H$. There are dimensional arguments $[70,71]$ that predict $\beta / H \sim$ $4 \log \left(M_{p} / \Lambda_{Q C D, i}\right)$, although these arguments make specific assumptions about the potential. In more recent work, some authors $[88,90]$ have attempted to estimate it using first-order chiral effective theories and the Polyakov-Nambu-Jona-Lasinio models which motivates a $\beta / H$ of $\mathcal{O}\left(10^{4}\right)$. These studies claim a large range of values with no consensus reached on the precise order of the scaled inverse timescale. Under these circumstances, our signal projections will consider both extremes of the parameter space where, $\beta / H \sim 10-10^{4}$. A more realistic scenario may exist in between both cases.

\subsubsection{Production of Gravitational Waves}

Gravitational waves are produced with contributions from different components of the SFOPT's evolution. It is commonplace to parameterize the spectral energy density in gravitational waves by [115]

$$
\Omega_{\mathrm{GW}}(f) \equiv \frac{1}{\rho_{c}} \frac{d \rho_{\mathrm{GW}}(f)}{d \log (f)}
$$

where $\rho_{c}=3 H^{2} /(8 \pi G)$ is the critical energy density. The total gravitational wave signal is a linear combination of three leading contributions:

$$
h^{2} \Omega_{\mathrm{GW}} \approx h^{2} \Omega_{\phi}+h^{2} \Omega_{v}+h^{2} \Omega_{\text {turb }}
$$

Each component is scaled by its own unique efficiency factor, $\kappa$ (see Eq. 5.41) . The three leading-order contributions to the GW power spectrum are as follows:

- Scalar field contributions $\Omega_{\phi}$ : Caused by collisions of the bubble walls, the 
solutions being completely dependent on the scalar field configuration, with efficiency factor $\kappa_{\phi}=1-\alpha_{\infty} / \alpha[116,117]$.

- Sound wave contributions $\Omega_{v}$ : Sound waves within the plasma after bubble collision will produce $\beta / H$ enhanced gravitational waves, with efficiency factor $\kappa_{v} \propto \alpha_{\infty} / \alpha[118]$

- Magnetohydrodynamical contributions $\Omega_{B}$ : Turbulence within the plasma, left over from the sound wave propagation, will produce gravitational waves with efficiency factor $\kappa_{t u r b} \approx 0.1 \kappa_{v}[119]$.

The parameter $\alpha_{\infty}$ denotes the dividing line between the runaway regime $\left(\alpha>\alpha_{\infty}\right)$ and the nonrunaway regime $\left(\alpha<\alpha_{\infty}\right)$. Explicitly [51,73, 114],

$$
\alpha_{\infty}=\frac{\left(T_{h}^{n u c}\right)^{2}}{\rho_{R}}\left[\sum_{\text {bosons }} n_{i} \frac{\Delta m_{i}^{2}}{24}+\sum_{\text {fermions }} n_{i} \frac{\Delta m_{i}^{2}}{48}\right]
$$

for particles with $n_{i}$ degrees of freedom that obtain mass through the phase transition.

The exotic sectors have essentially massless degrees of freedom pre phase transition and pions with negligible masses post phase transition. Other composite particles, such as baryons, do gain a mass of the order of $\Lambda_{e x}$; this is, however, still much smaller than the order of $\rho_{R}$ leading to small $\alpha_{\infty}$ according to Eq. (5.40). ${ }^{4}$ Heavy standard sectors that undergo SFOPT for QCD feature no baryons due to all quarks being above their respective QCD scales. They do, however, feature glueballs that obtain a mass of the order of the SFOPT and, just as in the case for exotic sectors above, feature small $\alpha_{\infty}$.

Each component of the spectral energy density in Eq. (5.38) is proportional to a power of their respective efficiency factors $\kappa$. The relative strength of the efficiency

\footnotetext{
${ }^{4}$ It should also be noted that although free quarks cease to exist post phase transition in these exotic sectors, their masses are so light that they do not contribute relevant amounts to $\alpha_{\infty}$.
} 
factors is dependent on the ratio $\frac{\alpha_{\infty}}{\alpha}-$ since both $\alpha_{\infty}$ and $\alpha$ are parameterically small $[88,90]$, a range of possible scenarios can occur. Here, we discuss the two ends of this spectrum: pure runaway walls and pure nonrunaway walls. The former scenario with runaway bubble walls leads to the efficiency factors for the sound wave and magnetohydrodynamics (MHD) contributions being small and ensures GWs are dominantly produced from bubble collisions, $h^{2} \Omega_{\mathrm{GW}} \approx h^{2} \Omega_{\phi}$ : this is what we assume for the remainder of this section. In the latter case, bubbles are nonrunaway (but the bubble wall velocity is still $v_{w} \sim 1[51,120]$ ) such that nonbubble collision contributions are important - ultimately leading to significant changes to the GW profile. This case and the gravitational waves it produces are examined in Sec. 5.7. Intermediate results are of course possible and would feature profiles somewhere in between the two extremes.

The form of the GW energy density at the time of nucleation is given by [51]

$$
h^{2} \Omega_{\mathrm{GW}}^{*}=7.7 \times 10^{-2}\left(\frac{\kappa_{\phi} \alpha}{1+\alpha}\right)^{2}\left(\frac{H}{\beta}\right)^{2} S(f)
$$

where we use $v=1$ for runaway bubbles. Quantities such as $\Omega_{\mathrm{GW}}^{*}$ that are calculated at the time of nucleation are denoted with an asterisk, and they must then be evolved to relate to their values at the time of observation. $S(f)$ is the spectral shape function for the signal and a parametric from has been found through numerical simulations [117] of bubble wall collisions:

$$
S(f)=\frac{3.8\left(f / f_{p}\right)^{2.8}}{1+2.8\left(f / f_{p}\right)^{3.8}} .
$$

The peak frequency $f_{p}$ is a function of the temperature of the SM at the time of nucleation. The various hidden sectors can phase transition at different scales and 
therefore temperatures, causing a shift in the GW spectrum's peak frequency given by $[117]$

$$
f_{p}=3.8 \times 10^{-8} \mathrm{~Hz}\left(\frac{\beta}{H}\right)\left(\frac{T_{\gamma}}{100 \mathrm{GeV}}\right)\left(\frac{g_{*}}{100}\right)^{\frac{1}{6}}
$$

where $g_{*}$ is calculated using Eq. (5.34), although, due to the lack of substantial reheating into the hidden sectors, the SM contribution is dominant.

Now that the framework has been laid out for the creation of GW from a single SFOPT, we generalize to multiple sectors going under independent, coherent SFOPT. In the models presented in this paper, we consider a subset of $N$ hidden sectors that undergo a phase transition at a SM temperature of $T_{\gamma}^{i}$. As the GWs propagate in free space, the energy density and frequency spectrum, at the time of production $\Omega_{\mathrm{GW}}^{*}(f)$, will redshift to today's value $\Omega_{\mathrm{GW}}^{0}(f)=\mathcal{A} \Omega_{\mathrm{GW}}^{*}\left(\left(a_{0} / a\right) f\right)$. The redshifting factor $\mathcal{A}$ accounts for the redshifting of both $\rho_{\mathrm{GW}}$ and $\rho_{c}[51,121]$,

$$
\mathcal{A} \equiv\left(\frac{a}{a_{0}}\right)^{4}\left(\frac{H}{H_{0}}\right)^{2}
$$

where $a\left(a_{0}\right)$ and $H\left(H_{0}\right)$ are the scale factor and Hubble constant at the time of nucleation (observation), respectively. Assuming that the sectors are completely decoupled before and after their respective SFOPT, the total GW signal that would be measured today is given by the coherent sum

$$
\Omega_{\mathrm{GW}}=\sum_{i}^{N} \mathcal{A}^{i} \Omega_{\mathrm{GW}}^{i, *}\left(\left(a_{0} / a\right)_{i} f\right) .
$$

We assume that the parameters of the SFOPT do not differ between sectors: the relativistic degrees of freedom, phase transition rate, and the dark QCD scale, are all similar. This makes the redshifting factor $\mathcal{A}^{i}$ independent of sector number. Applying this to the standard reheating scenario of $N$ naturalness, introduced in 5.4 , we 


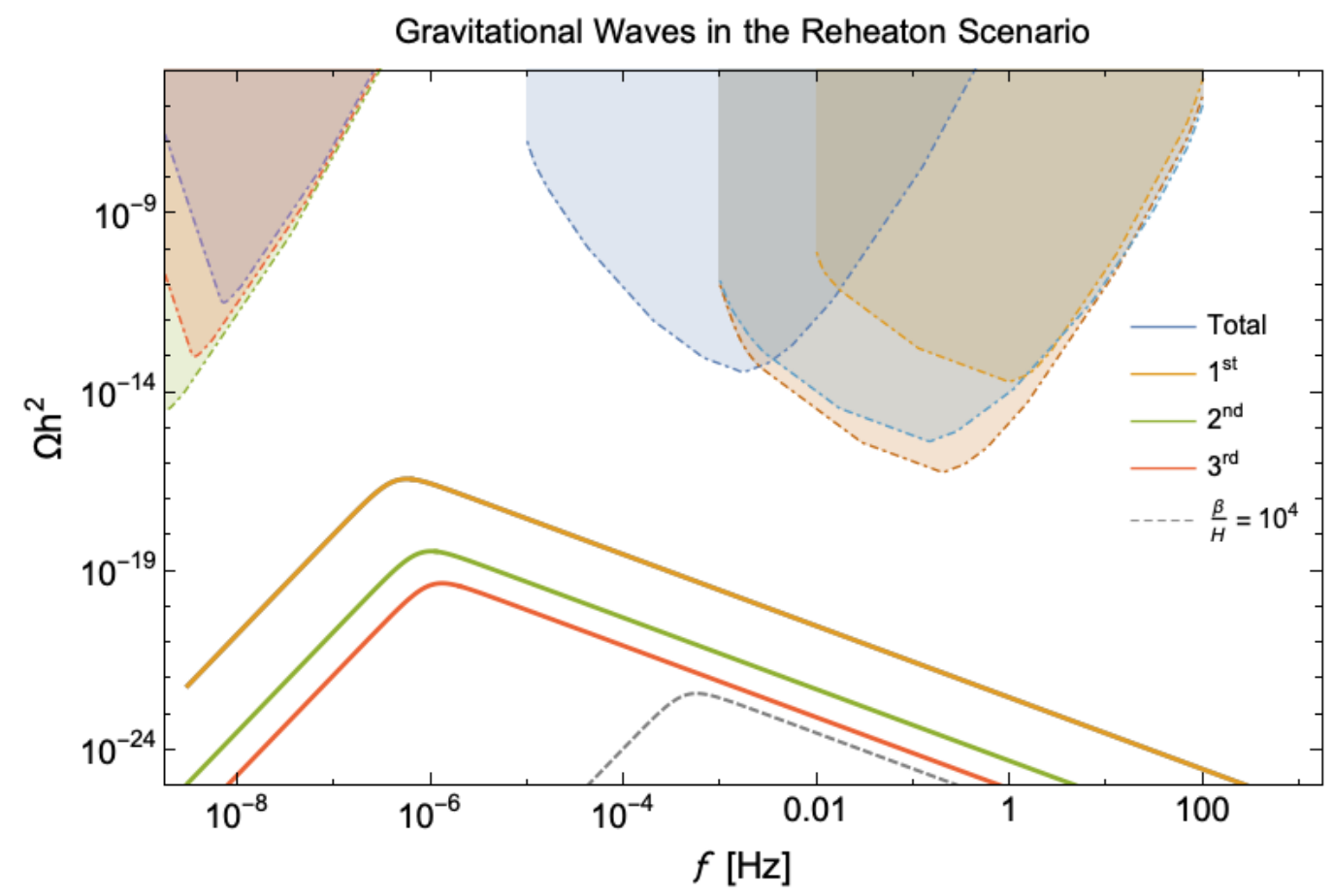

Figure 5.1: Gravitational wave spectral energy density (solid curves) for standard $N$ naturalness using the scalar reheaton model of section 5.4. The curve corresponding to the sum of the sectors is approximately equal to the $i=1$ curve. All contributions are assumed to be purely from bubble collisions $\Omega_{\phi}$. The colored solid lines use $\beta / H=10$ whereas the dashed gray line is the total contribution of all sectors for $\beta / H=10^{4}$ (the sum of all sectors is roughly equal to the $i=1$ curve and sectors beyond the first are below the range of this plot). The shaded dashed curves are the power law noise curves [47] calculated from expected sensitivity as described in Section 5.6.2. The ones on the right are space-based interferometers: LISA [44] (blue), DECIGO [17] (light blue), BBO [45] (red). The ones on the left are for the pulsar timing array SKA [52] for exposure time of 5-years (purple), 10-years (orange), and 20-years (green).

get GW signals as seen in Fig. 5.1. Plotted are the individual contributions to the signal from each phase transitioned sector, as well as the coherent sum of all sectors. Future GW interferometers and pulsar timing array sensitivity curves are shown in comparison to the signal. The sensitivity curves are interpreted as the region of possible detection if intersected with the GW signal, and the construction of these curves is detailed in Section 5.6.2. Notice that the total signal is dominated by the first 
sector's contribution. This is caused by the quartic temperature ratio suppression in Eq. (5.33) and the large temperature gaps between adjacent sectors. Such a suppression leads to standard Nnaturalness evading future detector thresholds by a few orders of magnitude in units of energy density.

This is not the case if we consider more generalized reheating scenarios. Once the restriction that sectors with small Higgs masses are preferentially reheated has been lifted, we can explore a much more vast landscape of hidden sectors than are allowed in the reheaton case. Here, we construct several different scenarios that are both detectable and demonstrate a variety of gravitational wave profiles. Specifically, we explore benchmarks that lead to a deviation in the peak behaviour of the total GW signal (the superposition of stochastic GW from individual SFOPT) from a standard power law signal.

It should be noted that the key phenomenological constraint on all of these models is $\Delta N_{e f f}$, giving us a maximum allowed temperature ratio (when compared to the SM) for each reheated hidden sector: Eq. (5.31) shows the maximum temperature ratios for specific numbers of additional hidden sectors. Due to the rather harsh scaling of the GW strength $\alpha$ with the temperature ratio shown in Eq. (5.35), we take the optimistic approach of keeping the temperature ratio as high as allowed by CMB data for all of the hidden sectors.

In the following, we focus on heavy standard sectors - pure Yang-Mills sectors with much heavier particles (specifically quarks) and, as shown in Sec. 5.3, the SFOPT these entail. The reason for this arises from Eq. (5.43): every exotic sector features a phase transition that occurs at $\Lambda_{e x} \sim 90 \mathrm{MeV}$. If we maximize the allowed temperature ratio, this gives us a $(\mathrm{SM})$ photon temperature $T_{\gamma}$ that places our signal directly in the frequency void between the detection region of pulsar timing arrays and space-based interferometers (see Sec. 5.6.2). The location of the peak can be 
changed by dropping the temperature ratio, but the adjustment required to end up with a signal with an appropriate peak frequency makes the overall signal too weak to detect. As shown in Sec. 5.3, standard sectors can have much higher temperature phase transitions. As such, maintaining the maximum allowed temperature ratio between the hidden sector(s) and the SM gives a much larger photon temperature and a proportionally larger peak frequency; ultimately allowing for detection by space-based interferometers.

There are four scenarios that we examine, with key parameters presented in Table 5.1.

- Maximized signal: A single additional heavy hidden sector reheated to a temperature that saturates current experimental bounds. The SM photon bath temperature at the time of the hidden sector PT is $87 \mathrm{GeV}$. In the Nnaturalness framework this is equivalent to reheating a standard sector with $i \sim 10^{16}$ up to the maximum allowed temperature ratio.

- Large split scenario: A scenario where two additional hidden sectors have been reheated - these sectors have Higgs VEVs that are split by a factor of

$$
\frac{v_{h 1}}{v_{h 2}}=\sqrt{10^{3}}
$$

This results in a difference in the scale of the SFOPTs leading to the SM photon bath temperature changing a large amount during the time between the PTs. This, in turn, leads to a large separation in the peak frequency of their gravitational wave signals. In the $N$ naturalness framework this is equivalent to reheating two standard sectors, one with $i \sim 10^{12}$ and another with $i \sim 10^{15}$ up to the maximum allowed temperature ratio. 


\begin{tabular}{|c|c|c|c|c|}
\hline \multicolumn{5}{|c|}{ Parameters for multi-hidden sector benchmarks } \\
\hline \multicolumn{5}{|c|}{ Maximized signal } \\
\hline Sector & $\begin{array}{l}\text { Higgs } \\
(\mathrm{GeV})\end{array}$ & $\Lambda_{Q C D}^{A S}(\mathrm{GeV})$ & $T_{\gamma}(\mathrm{GeV})$ & Index \\
\hline 1 & $24.6 \times 10^{9}$ & 38.6 & 87.7 & $10^{16}$ \\
\hline \multicolumn{5}{|c|}{ Large split } \\
\hline Sector & $\begin{array}{l}\text { Higgs } \\
(\mathrm{GeV})\end{array}$ & $\Lambda_{Q C D}^{A S}(\mathrm{GeV})$ & $T_{\gamma}(\mathrm{GeV})$ & Index \\
\hline 1 & $246 \times 10^{6}$ & 10.8 & 30.3 & $10^{12}$ \\
\hline 2 & $7.8 \times 10^{9}$ & 28.1 & 78.6 & $10^{15}$ \\
\hline \multicolumn{5}{|c|}{ Medium split } \\
\hline Sector & $\begin{array}{l}\text { Higgs } \\
(\mathrm{GeV})\end{array}$ & $\Lambda_{Q C D}^{A S}(\mathrm{GeV})$ & $T_{\gamma}(\mathrm{GeV})$ & Index \\
\hline 1 & $246 \times 10^{6}$ & 10.8 & 30.3 & $10^{12}$ \\
\hline 2 & $778 \times 10^{6}$ & 14.9 & 41.6 & $10^{13}$ \\
\hline \multicolumn{5}{|c|}{ Five sector } \\
\hline Sector & $\begin{array}{l}\text { Higgs } \\
(\mathrm{GeV})\end{array}$ & $\Lambda_{Q C D}^{A S}(\mathrm{GeV})$ & $T_{\gamma}(\mathrm{GeV})$ & Index \\
\hline 1 & $246 \times 10^{6}$ & 10.8 & 38.8 & $10^{12}$ \\
\hline 2 & $426 \times 10^{6}$ & 12.6 & 45.2 & $3 \times 10^{12}$ \\
\hline 3 & $778 \times 10^{6}$ & 14.9 & 53.3 & $10^{13}$ \\
\hline 4 & $1.3 \times 10^{9}$ & 17.3 & 62.1 & $3 \times 10^{13}$ \\
\hline 5 & $2.5 \times 10^{9}$ & 20.5 & 73.3 & $10^{14}$ \\
\hline
\end{tabular}

Table 5.1: Outline of parameters used for the various multi-hidden-sector scenarios. The Higgs VEV is the VEV for the given additional sector, $\Lambda_{Q C D}^{A S}$ is the QCD phase transition in the additional sector, and $T_{\gamma}$ is the temperature of the SM photon bath when the SFOPT occurs in the additional sector. The index indicates the equivalent sector from the $N$ naturalness model (Eq. (5.4)). It should be noted that although the various sectors undergo phase transitions at different temperatures, they are all assumed to be reheated to the same initial temperature. 
- Medium split scenario: Similar to the previous case: these sectors have Higgs VEVs that are split by a factor of

$$
\frac{v_{h 1}}{v_{h 2}}=\sqrt{10},
$$

resulting in a much smaller difference in the peak frequency of their gravitational wave signals. In the $N$ naturalness framework this is equivalent to reheating two standard sectors, one with $i \sim 10^{12}$ and another with $i \sim 10^{13}$ up to the maximum allowed temperature ratio.

- Five sector scenario: Five sectors are reheated to the maximum allowed temperature ratio, each with VEVs that are

$$
\left(v_{h i}\right) /\left(v_{h(i+1)}\right) \sim \sqrt{3}
$$

larger than the previous sector.

In all cases where multiple sectors are reheated, we assume for simplicity that all the hidden sectors are reheated to the same temperature.

The GW results of these cases are presented in Fig. 5.2. In all cases, the summed GW signal is detectable by one or more proposed interferometers. When changing the assumptions on $\beta / H$, the scenarios in Fig. 5.2 are still detectable for values ranging between $\mathcal{O}(1)$ and $\mathcal{O}(100)$. As $\beta / H$ increases (decreases) the peak frequency moves to higher (lower) frequencies, dictated by Eq. (5.43), whereas the amplitude decreases (increases) shown in Eq. (5.41).

The frequency dependence in Eq. (5.42) takes the form of $f / f_{p}$, this causes a cancellation between the redshifting factors. As multiple sectors phase transition at different times, and therefore different SM photon temperatures, the peaks will 

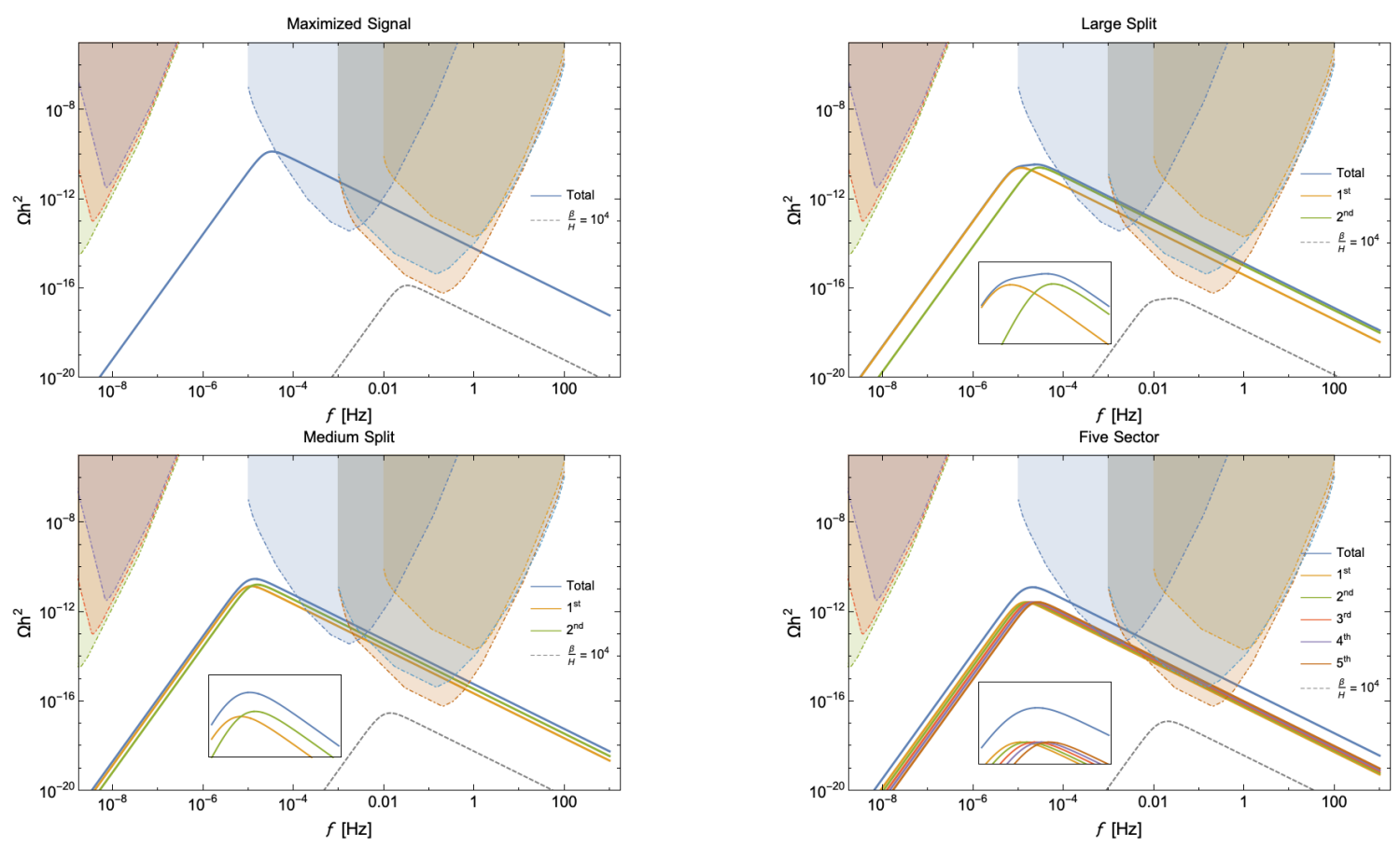

Figure 5.2: Gravitational wave spectral energy density for the various scenarios found in Table 5.1. All contributions are assumed to be purely from runaway bubble collisions $\Omega_{\phi}$. The colored solid lines use $\beta / H=10$ where as the dashed gray line is the total contribution of all sectors for $\beta / H=10^{4}$. The inset is a closer look at the region around the peaks for the $\beta / H=10$ case. The shaded curves are the same as Fig. 5.1.

shift relative to each other, purely from the linear temperature dependence of the peak frequency $f_{p} \sim T_{\gamma}$ given in Eq. (5.43). This is seen in Fig. 5.2, where the spectrum peaks are shifted causing a peak broadening of the summed spectrum. The broadening can be substantial if the hidden sectors transition between a large gap of time (temperature). Eventually, a temperature limit will be reached where two (or multiple) distinct peaks will be visible, provided that the amplitudes are comparable.

\subsubsection{Detection of Stochastic Graviational Waves}

A stochastic gravitational wave background could be detectable if the signal-to-noise ratio (SNR) is above some threshold value, $\rho>\rho_{t h}$, dictated by the capabilities of fu- 
ture interferometers and pulsar timing arrays (PTAs). These interferometers or PTAs quote their experimental sensitivies in terms of spectral noise curves, $S_{\text {eff }}(f)$, which can be translated into units of energy density through $h^{2} \Omega_{\mathrm{eff}}(f)=\frac{2 \pi^{2}}{3 H^{2}} f^{3} S_{\text {eff }}(f)$. If the experiment uses a single (multiple) detector, the autocorrelated (cross-correlated) SNR is used in comparing to the threshold value $\rho_{t h}$. The autocorrelated and crosscorrelated SNR are explictly given as [43],

$$
\begin{gathered}
\rho^{2}=\mathcal{T} \int_{f_{\min }}^{f_{\max }} \mathrm{d} f\left(\frac{h^{2} \Omega_{\mathrm{GW}}(f)}{h^{2} \Omega_{\mathrm{eff}}(f)}\right)^{2} \quad \text { (autocorrelated) } \\
\rho^{2}=2 \mathcal{T} \int_{f_{\min }}^{f_{\max }} \mathrm{d} f\left(\frac{h^{2} \Omega_{\mathrm{GW}}(f)}{h^{2} \Omega_{\mathrm{eff}}(f)}\right)^{2} \quad \text { (cross-correlated) }
\end{gathered}
$$

where $\mathcal{T}$ is the exposure time of the experiment. The integration covers the entire broadband range of frequencies $\left(f_{\min }, f_{\max }\right)$. LISA [44] and B-DECIGO [17] are proposed to be single-detector interferometers, whereas BBO [45] and DEICIGO [46] would be built from an array of multiple interferometers. GW signals produced from an early cosmological phase transition would be seen as a stochastic background. Assuming that the GW follows a power law background in frequency, it is commonplace to quote the power law integrated (PLI) sensitivity curves [47]. The PLI curves are constructed using information from the power law form of the signal,

$$
h^{2} \Omega_{\mathrm{GW}}(f)=h^{2} \Omega_{\gamma}\left(\frac{f}{f_{\mathrm{ref}}}\right)^{\gamma}
$$

where $\gamma$ is the spectral index of the power law, and $f_{\text {ref }}$ is an arbitrary reference frequency which has no effect on the PLI sensitivities. $h^{2} \Omega_{\gamma}$ is the energy density calculated using Eq. (5.49) with spectral index $\gamma$ and reference frequency $f_{\text {ref }}$. The method of calculating the PLI curves involves plotting $h^{2} \Omega_{\mathrm{GW}}(f)$, using Eq. (5.50), for various spectral indices $\gamma$ and for some fixed threshold value of $\rho_{t h}$. Each curve 
will lay tangent to the PLI curve, more formally,

$$
h^{2} \Omega_{\mathrm{PLI}}=\max _{\gamma}\left[h^{2} \Omega_{\gamma}\left(\frac{f}{f_{\mathrm{ref}}}\right)^{\gamma}\right] .
$$

The spectral noise curves used to create the PLI curves shown in Figs. 5.1 and 5.2 were taken from $[17,48-51]$ for the interferometers and $[51,52]$ for the Square Kilometer Array (SKA) pulsar timing array. We have assumed an observation time of $\mathcal{T}=4$ years for the interferometers and $\mathcal{T}=5,10,20$ years for the various stages of SKA. In the case of the PTA experiments, the sensitivity curves are dependent on how frequently the pulsar's timing residuals, $\delta t$, are measured. When using Eq. (5.49) to construct the PLI curves for SKA, the upper integration bound is inversely proportional to pulsar's timing residual, $f_{\min }=1 / \delta$. In this work, it is assumed that $\delta t=14$ days, but this may underestimate the capabilities of SKA as well as the cadences of the pulsar populations. If the timing residuals are lowered the maximum frequency reach of SKA increases, and the corresponding PLI curves in Figs. 5.1 \& 5.2 are shifted to the right, possibly giving the PTAs sensitivity to some of the scenarios considered here.

\subsection{Nonrunaway phase transitions}

If the phase transition occurs in the nonrunaway regime $\left(\alpha_{\infty}>\alpha\right)$, the dominant contributions to the GW energy densities are given by the sound wave $h^{2} \Omega_{v}$ and MHD $h^{2} \Omega_{\text {turb }}$ components [51],

$$
h^{2} \Omega_{\mathrm{GW}} \approx h^{2} \Omega_{v}+h^{2} \Omega_{\text {turb }}
$$

The new contributions to the GW energy density take on a different form from 

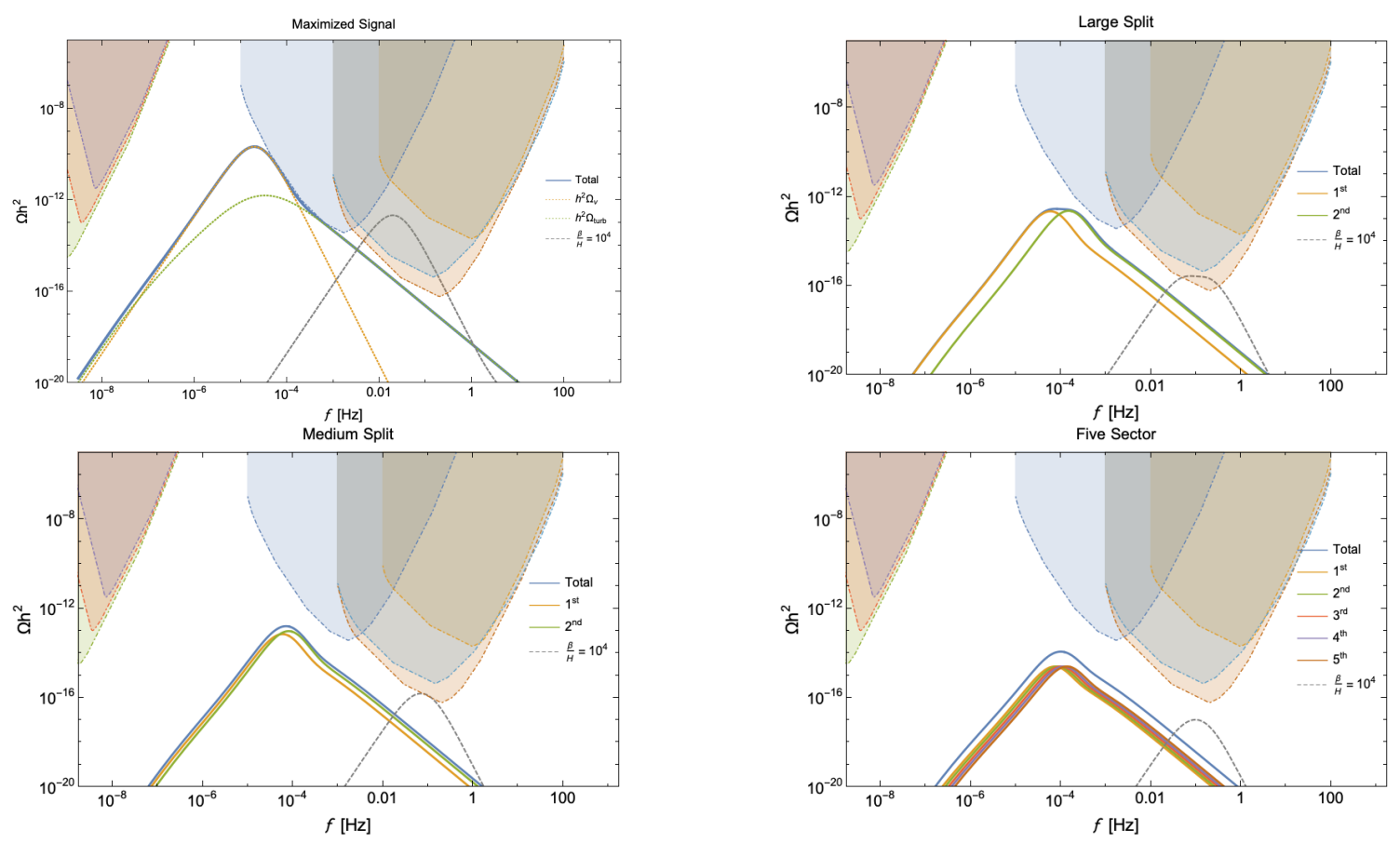

Figure 5.3: Gravitational wave spectral energy density for the various scenarios found in Table 5.1. All contributions are assumed to be from a nonrunaway phase transition with terminal velocity $v=0.95$. The coloured solid lines use $\beta / H=10$ whereas the dashed grey line is the total contribution of all sectors for $\beta / H=10^{4}$ . The top left figure shows the individual sound wave and MHD contributions. The shaded curves are the same as Fig. 5.1. In contrast to the runaway case, most scenarios evade the projected sensitivities.

Eq. (5.41) [73],

$$
\begin{array}{r}
h^{2} \Omega_{v}^{*}=1.6 \times 10^{-1} v\left(\frac{\kappa_{v} \alpha}{1+\alpha}\right)^{2}\left(\frac{H}{\beta}\right)^{1} S_{v}(f), \\
h^{2} \Omega_{\text {turb }}^{*}=2.01 \times 10^{1} v\left(\frac{\kappa_{\text {turb }} \alpha}{1+\alpha}\right)^{3 / 2}\left(\frac{H}{\beta}\right)^{1} S_{\text {turb }}(f) .
\end{array}
$$

Unlike the runaway case, we do not assume $v=1$ due to the bubbles reaching a terminal velocity. The MHD efficiency factor is a fraction of the sound waves, $\kappa_{\text {turb }}=\varepsilon \kappa_{v}$. Current simulations have motivated a range of $\varepsilon \sim 0.05-0.10$ [73], where we take the optimistic case of $\varepsilon=0.10$. Both contributions have unique 
spectral shapes, given to be [51],

$$
\begin{array}{r}
S_{v}(f)=\left(f / f_{p, v}\right)^{3}\left(\frac{7}{4+3\left(f / f_{p, v}\right)^{2}}\right)^{7 / 2}, \\
S_{\text {turb }}(f)=\frac{\left(f / f_{p, t u r b}\right)^{3}}{\left(1+f / f_{p, t u r b}\right)^{11 / 3}(1+8 \pi(f / H))} .
\end{array}
$$

The MHD energy density has a spectral shape dependent on the Hubble rate at the time of nucleation, $H$. Similar to the scalar spectral shape in Eq. (5.42), the frequencies are scaled by their respective temperature-dependent peak frequency:

$$
\begin{aligned}
f_{p, v} & =1.9 \times 10^{-5} \mathrm{~Hz} \frac{1}{v}\left(\frac{\beta}{H}\right)\left(\frac{T_{\gamma}}{100 \mathrm{GeV}}\right)\left(\frac{g_{*}}{100}\right)^{\frac{1}{6}}, \\
f_{p, t u r b} & =2.7 \times 10^{-5} \mathrm{~Hz} \frac{1}{v}\left(\frac{\beta}{H}\right)\left(\frac{T_{\gamma}}{100 \mathrm{GeV}}\right)\left(\frac{g_{*}}{100}\right)^{\frac{1}{6}} .
\end{aligned}
$$

We evolve the frequencies and energy densities with the same redshift factors, Eq. (5.44) from Sec 5.6. Fig. 5.3 shows the sum of the GW energy density for the scenarios in Table 5.1, but instead for the nonrunaway case, with a terminal velocity of $v=0.95$. For this case we see that the spectra tend to be shifted to lower frequencies and are more likely to fall in the gap between the interferometers and the pulsar-based detectors. On the other hand, larger values of $\beta / H$ increase the typical frequency, so this case becomes more sensitive in some scenarios to values of $\beta / H$ on the larger end of the considered range.

In the sound wave case, these parameterizations are extracted from simulations with $\beta / H<100$ corresponding to a long-lasting sound wave component. For high $\beta / H$ the transition timescale from sound wave to MHD turbulence is much shorter than the Hubble time. When estimating the model expectations for $\beta / H=10^{4}$, we enter a regime at which Eq. (5.53) may be overestimating the sound wave contribution. 
Investigations of this regime have been done in [122-124]. This effect, however, only affects the amplitude of the signal, and our work focuses on the unique spectral shapes that are formed in these models. Therefore we project our results for high $\beta / H$ in Fig. 5.3 to motivate the novel spectral profiles.

Finally, we note that because the sound wave and MHD contributions have different spectral shapes, the overall spectrum has a kink at a frequency above the peak. In the top left panel of Fig. 5.3, we show the two contributions separately in addition to their sum to highlight this effect.

\subsection{Conclusion}

As detection capabilities increase, gravitational wave signals continue to grow in importance as phenomenological signatures that can offer us a unique glimpse into the universe as it was in the early epochs. The space-based interferometers planned for the next generation of GW experiments will be sensitive enough to begin searching for signals of the cataclysmic disruption of space-time due to SFOPT. As we inch closer to these measurements becoming available, it becomes important to develop ways to analyze and understand this data.

Here, we examined scenarios, including Nnaturalness, that involve multiple hidden sectors and calculated the GW profiles present. Our GW projections demonstrate that although $N$ naturalness with the reheaton scenario presented in [57] is not projected to be detectable in the near future, more generalized scenarios with multiple hidden sector SFOPTs are in an observable region and will begin to be probed by next-generation space experiments. Both cases feature important parts of their GW signals in the void between frequencies detectable by pulsar timing arrays and spacebased interferometers - providing theoretical impetus for new experiments capable 
of probing this region of frequency space.

Further, our results provide a framework for understanding and using GW signals in two different ways: first as a unique signal for specific theories featuring multiple SFOPTs and also as a challenge to broaden the understanding of GW detector sensitivity.

In the former case, this demonstrates the power of GW signals to probe deep into the unknown arena of complex hidden sectors. Individual SFOPTs are understood to create GWs that are assumed to follow an approximate power law. If a model predicts the presence of two, five, or more additional sectors, or features a single extra sector with multiple PTs, deviations from a standard power law can occur. The multiple transitions that occur in the models outlined here create signals that follow this trend: although the individual GWs do obey approximate power laws, their sum does not - leading to a unique signal indicating so-called dark complexity. Explicitly, a broadening or distortion of the signal around the peak frequency, precisely where the signal has the most energy, could point to a multi-SFOPT scenario and gently guide us in the direction of multiple hidden sectors.

Shifting to the other part of our framework, our results leads to the question "how well can experiments probe non-power-law signals?" For frequency ranges away from the peak of the total, GW signals the quoted detection thresholds should hold: the signals fall off as a power law to a very good approximation. However, for areas around the peak frequency the answer is less clear; the PLI curves are built under the assumption of a power law. Work has been done [125] in examining GW signals using peak amplitudes and peak frequencies as the defining observables: this is rooted in the assumption that GW signals have a model-independent spectral shape around peak frequencies. However, our results indicate that this assumption of model independence cannot hold for all cases: sectors with similar (but different) transition 
temperatures can create either peak broadening or multihump features that differ significantly from a standard power law shape. This points to the need for future work to better understand where the power law approximation breaks down and how this affects detection prospects for the next generation of GW detectors. 


\section{Chapter 6}

\section{Higgs Portal From The}

\section{Atmosphere To Hyper-K}

A Standard Model gauge singlet scalar that mixes with the Higgs boson, sometimes also referred to as the "dark Higgs", is a simple new physics candidate. It has been introduced for exploring the dark universe [53,55, 126-128], facilitating baryogengesis mechanisms [129-132], precision physics of the Standard Model [133,134], and, perhaps, naturalness [135]. In its minimal incarnation, the Higgs portal scalar is produced in laboratories and decays into Standard Model particles via the same mixing parameter with the Higgs boson. These makes it a well-motivated and well-defined target of searches in a number of experiments. Constraints have been set for a wide range of its mass $[54,136,137]$. In particular, if the scalar is lighter than $\sim \mathrm{GeV}$, leading constraints come from the measurement of rare $K$ and $B$ meson decays where the mixing parameter must be smaller than $\sim 10^{-3}$.

Recently, the Higgs portal scalar has been revisited for understanding a new experimental finding. In 2016-18, the KOTO experiment at J-PARC performed a search for the flavor-changing decay process $K_{L} \rightarrow \pi^{0} \nu \bar{\nu}$, in final states with two 
energetic photons plus missing transverse momentum. It was originally reported that four candidate events were identified whereas the Standard Model predicts nearly none [138-140]. Although in a more recent analysis by the KOTO collaboration, the significance of this excess is substantially reduced [141], it has triggered the exploration of a variety of potential new physics both heavy and light. Among them, a light Higgs portal scalar $\phi$ stands out as the simplest candidate [142] (see also [143-145]). The signal can be explained as $K_{L} \rightarrow \pi^{0} \phi$ decay where $\phi$ is long lived and escapes the detector. The potentially relevant parameter space, corresponding to a $\phi$ mass between $100-200 \mathrm{MeV}$, and $\phi$-Higgs mixing parameter of (a few) $\times 10^{-4}$, is a blindspot of existing searches at the intensity frontier. The most direct cross check is the isospin related decay mode, $K^{+} \rightarrow \pi^{+} \phi \rightarrow \pi^{+}+$invisible, which can be constrained by the $K^{+} \rightarrow \pi^{+} \nu \bar{\nu}$ measurement. Indeed, this channel has been searched for at the E949 [146] and NA62 [147-149] experiments where upper limits are set on the mixing parameter of the Higgs portal scalar. However, both limits feature a gap when the scalar mass is around the pion mass, due to the enormous $K^{+} \rightarrow \pi^{+} \pi^{0}$ background. In this mass window, the best upper limit on the mixing parameter is set by an early beam dump experiment, CHARM [150], in the search for displaced decay of $\phi$.

This comparison points to a direction to proceed. In order to cover the Higgs portal scalar in the above blindspot, one should resort to appearance experiments hunting the visible decay of long lived $\phi$ particles rather than disappearance experiments searching for $\phi$ as missing momentum. As a further useful observation, with a mixing with the Higgs boson $\sim 10^{-4}$, the decay length of a Higgs portal scalar is of order hundreds of kilometers, and even longer if boosted. This gives motivation to imagine large experiments operating at length scales beyond those beam-based ones built entirely within the laboratories.

In this chapter, we propose using a nature-made experimental setup to probe 
the Higgs portal scalar $\phi$. It utilizes cosmic rays as the beam, earth's atmosphere as the target, and earth itself as the shielding region. In this picture, $\phi$ particles originate from the decay of kaons, with the latter being abundantly produced in the cosmic-ray-atmosphere fixed-target collisions, together with charged pions that make the atmospheric neutrinos [8]. If long lived enough, the $\phi$ particles travel a long distance across the earth before decaying inside a human-made detector. We focus on the Hyper-Kamiokande (Hyper-K) experiment [151] which, at least for the foreseeable future, has the largest detector volume and a suitably low energy threshold to capture the scalar decays.

The Higgs portal scalar is defined as a mass eigenstate and a linear combination of a Standard Model gauge singlet $s$ and the Higgs boson $h$,

$$
\phi=\cos \theta s+\sin \theta h,
$$

where $\theta$ is a real mixing parameter. Such a singlet-Higgs mixing can be generated by adding to the standard model Lagrangian a Higgs portal interaction term $\mu s H^{\dagger} H$. After the electroweak symmetry breaking, it generates a bilinear term that allows the singlet scalar to mix with the Higgs boson. This corresponds to the minimal scenario of scalar portal (BC4) considered in the community report Ref. [54]. For simplicity, we proceed with the following discussions using the above phenomenological parametrization.

The cosmic rays near us are dominated by protons while the elements in the earth's atmosphere are dominated by nitrogen and oxygen, comprised of equal numbers of protons and neutrons. We simulate fixed target proton-proton and protonneutron collisions using PYTHIA 8 [152] for various incoming proton energies, which is further convoluted with the incoming cosmic proton spectrum [153] to derive the 


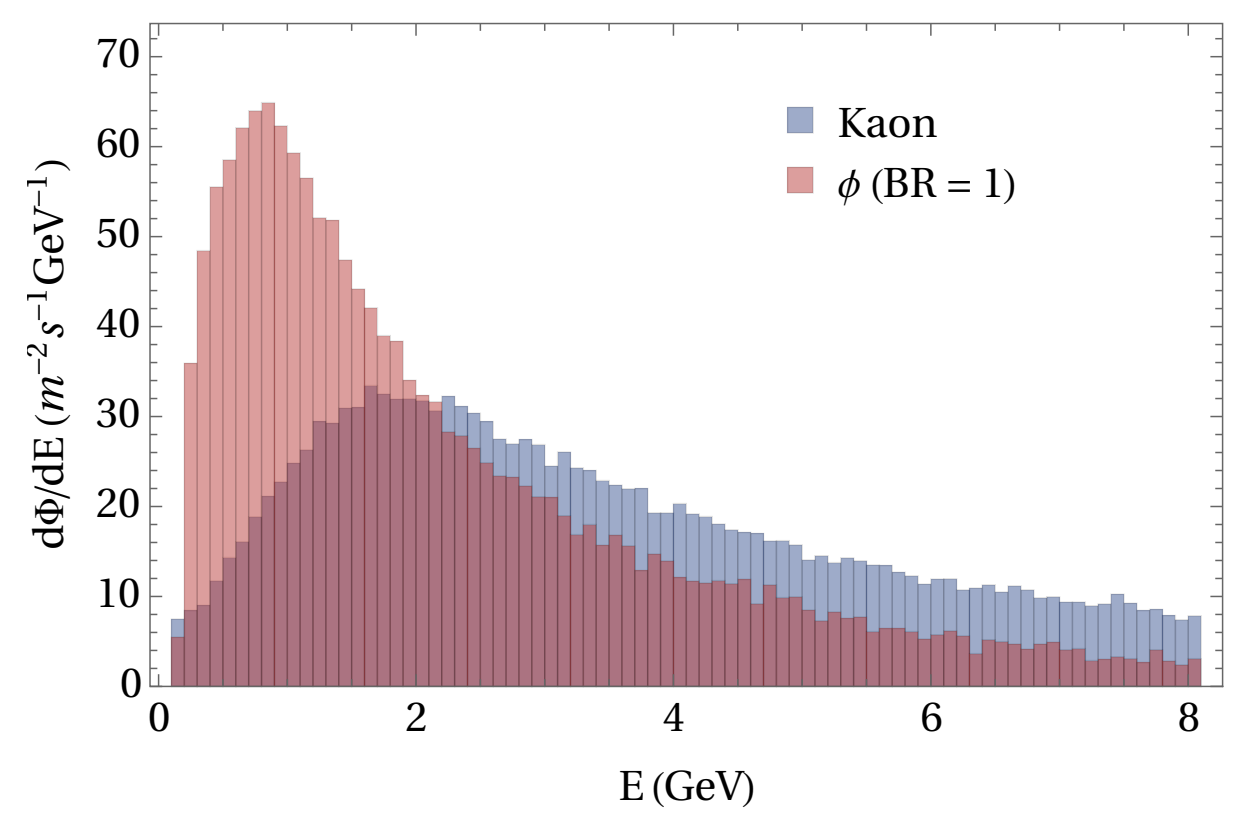

Figure 6.1: Energy distribution of atmospheric kaons ( $K^{ \pm}$and $K_{L}$ added together) and $\phi$ particles, for $m_{\phi}=150 \mathrm{MeV}$, obtained from the atmospheric simulation described in the text. For illustration purpose, the flux of $\phi$ has been rescaled by assuming the $K \rightarrow \pi \phi$ decay branching ratios are equal to 1 .

differential energy spectrum of kaons (most relevant for this study, $K^{ \pm}$and $K_{L}$ ), $d \Phi / d E_{K}$. Their sum is shown as the blue histogram in Fig. 6.1. The ratio of $K^{ \pm}$and $K_{L}$ particles is about $2: 1$, as expected.

The $\phi$ particles are produced from rare kaon decays, $K^{ \pm} \rightarrow \pi^{ \pm} \phi$ and $K_{L} \rightarrow \pi^{0} \phi$. The corresponding branching ratios are [154-157]

$$
\begin{aligned}
& \operatorname{Br}\left(K^{ \pm} \rightarrow \pi^{ \pm} \phi\right) \simeq \frac{9 \tau_{K^{ \pm}}\left|V_{t s} V_{t d}^{*}\right|^{2} G_{F}^{3} m_{t}^{4} m_{K^{ \pm}}^{2} p_{\phi \mathrm{CM}} \theta^{2}}{2048 \sqrt{2} \pi^{5}} \\
& \operatorname{Br}\left(K_{L} \rightarrow \pi^{0} \phi\right) \simeq \frac{9 \tau_{K_{L}}\left[\operatorname{Re}\left(V_{t s} V_{t d}^{*}\right)\right]^{2} G_{F}^{3} m_{t}^{4} m_{K^{ \pm}}^{2} p_{\phi \mathrm{CM}} \theta^{2}}{2048 \sqrt{2} \pi^{5}}
\end{aligned}
$$

where the decay momentum in the center-of-mass (CM) frame is $p_{\phi \mathrm{CM}}=\lambda\left(m_{K}^{2}, m_{\pi}^{2}, m_{\phi}^{2}\right) / 2 m_{K^{ \pm}}$, and $\lambda$ is the Källén function. In the small $m_{\phi}$ limit, $\operatorname{Br}\left(K_{L} \rightarrow \pi^{0} \phi\right) / \operatorname{Br}\left(K^{ \pm} \rightarrow \pi^{ \pm} \phi\right) \simeq$ 
3.7 [158]. In the lab frame, the ratio of the final state $\phi$ energy to that of the kaon is

$$
\frac{E_{\phi}}{E_{K}}=\frac{E_{\phi \mathrm{CM}}}{m_{K}}+\frac{p_{\phi \mathrm{CM}}}{m_{K}} \sqrt{1-\frac{m_{K}^{2}}{E_{K}^{2}}} \cos \vartheta_{\mathrm{CM}}
$$

where $E_{\phi \mathrm{CM}}=\sqrt{p_{\phi \mathrm{CM}}^{2}+m_{\phi}^{2}}$ and $\vartheta_{\mathrm{CM}}$ is the relative angle between $\phi$ 's three-momentum in the kaon rest frame and the boost direction of the kaon. Because $K^{ \pm}$and $K_{L}$ are scalars, the angular $\phi$ distribution in their rest frame is isotropic. For given energy $E_{K}$, the values of $E_{\phi}$ distribute evenly between its extremes, corresponding to $\cos \vartheta_{\mathrm{CM}}= \pm 1$. The resulting differential flux of $\phi$ can be calculated using

$$
\begin{aligned}
\frac{d \Phi_{\phi}}{d E_{\phi}}=\sum_{K=K^{ \pm}, K_{L}} & \operatorname{Br}(K \rightarrow \pi \phi) \int_{E_{K \min }\left(E_{\phi}\right)}^{E_{K \max }\left(E_{\phi}\right)} d E_{K} \frac{d \Phi_{K}}{d E_{K}} \\
& \times \frac{m_{K}}{2 p_{\phi \mathrm{CM}} \sqrt{E_{K}^{2}-m_{K}^{2}}}
\end{aligned}
$$

where $E_{K \max \text { min }}$ is the largest (smallest) kaon energy that satisfies Eq. (6.3), for given $E_{\phi}$. In the limit $E_{K} \gg m_{K}, E_{K \max , \min } \simeq E_{\phi} m_{K} /\left(E_{\phi \mathrm{CM}} \mp p_{\phi \mathrm{CM}}\right)$. In Fig. 6.1, the red histogram shows the energy distribution of atmospheric $\phi$ particles, for $m_{\phi}=$ $150 \mathrm{MeV}$. Its energy is peaked $\sim 700 \mathrm{MeV}$.

It is worth pointing out that when simulating atmospheric $\phi$ production, we have to restrict the CM energy of $p p$ and $p n$ scatterings to be above $\sim 6 \mathrm{GeV}$ in order for the parton picture used by PYTHIA to be valid. We also neglected secondary reactions of the produced hadrons with the atmosphere before they decay, keeping in mind that the earth's atmosphere is dilute. Both processes could in principle result in more kaon (and thus $\phi$ particles) production. We made the above approximations for simplicity.

After being produced in the atmosphere, the $\phi$ particles can travel through the earth to decay inside human-made detectors, provided they have sufficiently long 


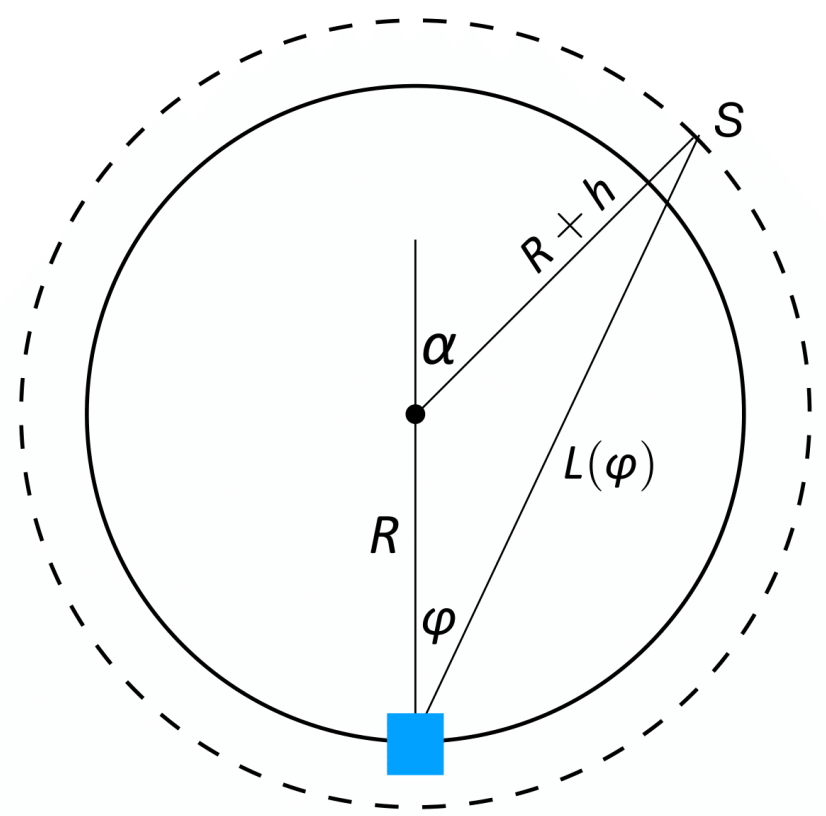

Figure 6.2: Geography of earth and detector. The blue box indicates the location of the Hyper-K detector. The dashed circle represents a sphere where the cosmicray-atmosphere reactions mainly occur that produce light $\phi$ particles. $h$ is given by the height of this sphere plus the depth of detector underground, and $\varphi$ is the zenith angle in view of the detector.

lifetimes. Clearly, the larger the detector the better to capture such a signal. Its energy threshold should be low enough to see sub-GeV energy deposits from the $\phi$ decay. These requirements led us to consider Hyper-K.

To calculate the $\phi$ flux at Hyper-K detector, we consider the geometric picture shown in Fig. 6.2. We assume all cosmic-ray-atmosphere reactions occur on a sphere with fixed height above the ground. This height plus the depth of the underground Hyper-K detector, denoted by $h$, is taken to be $10 \mathrm{~km}$. The angles $\varphi$ and $\alpha$ are related by

$$
\cos \alpha=[L(\varphi) \cos \varphi-R] /(R+h)
$$


where $L(\varphi)$ is the distance $\phi$ travels,

$$
L(\varphi)=R \cos \varphi+\sqrt{h^{2}+2 R h+R^{2} \cos ^{2} \varphi} .
$$

An infinitesimal area on the source sphere is

$$
d \mathcal{S}=2 \pi(R+h)^{2} d \cos \alpha=\frac{2 \pi(R+h) L(\varphi)^{2}}{L(\varphi)-R \cos \varphi} d \cos \varphi
$$

We assume cosmic ray showers on the earth atmosphere to be isotropic, and so is the resulting $\phi$ angular distribution within the hemisphere pointing towards the center of the earth. ${ }^{1}$ The flux of long-lived $\phi$ at the detector is related to its flux at the source (atmosphere) by a geometric factor,

$$
\left(\frac{d \Phi_{a}}{d E_{a}}\right)_{\text {detector }}=\left(\frac{d \Phi_{a}}{d E_{a}}\right)_{\text {source }} \int \frac{d \mathcal{S}}{2 \pi L(\varphi)^{2}}
$$

where the latter takes the form

$$
\int \frac{d \mathcal{S}}{2 \pi L(\varphi)^{2}}=\int_{0}^{\pi} \sin \varphi d \varphi \frac{(R+h)}{L(\varphi)-R \cos \varphi}
$$

If the Hyper-K detector volume is denoted by $V$, the event rate of long-lived $\phi$ particles decaying inside this volume is, regardless of its shape,

$$
R_{\text {event }}=V \int_{0}^{\pi} \sin \varphi d \varphi \frac{R+h}{L(\varphi)-R \cos \varphi}
$$

\footnotetext{
${ }^{1}$ It is a simplification that we assumed the atmospheric secondaries are produced isotropically inwards to the earth, which allows us to proceed the flux calculation analytically. We expect this approximation to be appropriate because we consider the high energy cosmic ray collisions (with center of mass energy above $6 \mathrm{GeV}$ ). As a result, the kaons and subsequently the $\phi$ particles are produced along the forward direction of the scatterings. In order for the $\phi$ particle to reach Hyper-K which is underground, the original cosmic ray direction cannot be tangential to the upper atmosphere.
} 


$$
\times \int d E_{\phi} \frac{d \Phi_{\phi} / d E_{\phi}}{\gamma \beta \tau_{\phi}} e^{-\frac{L(\varphi)}{\gamma \beta \tau_{\phi}}}
$$

where $\gamma$ is the boost factor of $\phi$ with energy $E_{\phi}$ and $\beta$ is the corresponding velocity. $d \Phi_{\phi} / d E_{\phi}$ is given by Eq. (6.4). The lifetime of $\phi$ is dictated by the Higgs portal. For mass of $\phi$ below twice the muon mass, it mainly decays into a $e^{+} e^{-}$pair. The corresponding decay length without boost factor is (assuming $m_{\phi} \gg m_{e}$ )

$$
\begin{aligned}
c \tau_{\phi} & =\frac{8 \pi}{\sqrt{2} G_{F} m_{e}^{2} m_{\phi} \theta^{2}} \\
& \simeq 30 \mathrm{~km}\left(\frac{0.15 \mathrm{GeV}}{m_{\phi}}\right)\left(\frac{5 \times 10^{-4}}{\theta}\right)^{2},
\end{aligned}
$$

where the benchmark values of $\theta$ and $m_{\phi}$ corresponds to the point indicated by the blue star in Fig. 6.3.

It is worth noting that the small electron mass appearing in the decay rate does not suppress the $\phi$ production rate (see Eq. (6.2)). ${ }^{2}$ Once produced from the atmosphere, it is able to penetrate the earth above deep underground detectors. In water Cherenkov detectors like Hyper-K, the final state $e^{+} e^{-}$manifest as a doublering signature, where the two rings originate from the same primary vertex of $\phi$ decay. We focus on fully contained events where the $\phi$ decay vertex emerges from inside the detector.

Our main result is shown in Fig. 6.3, in the $\theta$ versus $m_{\phi}$ plane. In the upper panel, the black solid, dashed, and dotted curves corresponds to observing 10, 100, and $1000 e^{+} e^{-}$pair events due to $\phi$ decay in the Hyper-K detector, after 10 years of data taking. To derive these curves, the volume of the Hyper-K detector used is $216 \times 10^{3} \mathrm{~m}^{3}($ diameter $=70.8 \mathrm{~m}$ and height $=54.8 \mathrm{~m})[151]$. Beyond the top and right

\footnotetext{
${ }^{2}$ This is in sharp contrast with the case of dark photon where the same parameter controls both the production and decay. In fact, Ref. [159] found that the atmospheric production cannot provide a competitive constraint for dark photon.
} 

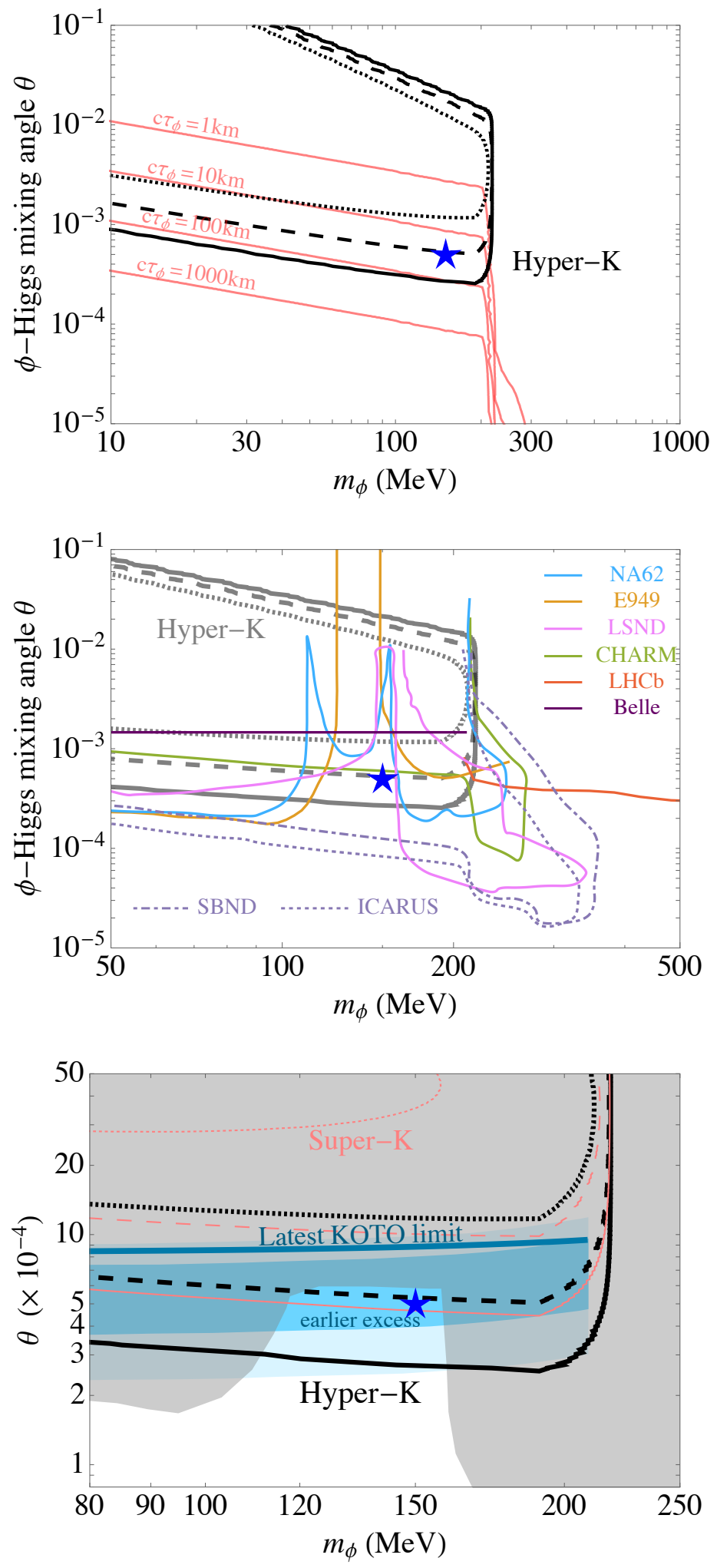

Figure 6.3: Caption next page. 
Figure 6.3: (Previous page.) Upper: Using Hyper-K detector to search for long lived Higgs portal scalar $\phi$ produced from the atmosphere. The (solid, dashed, dotted) black contours correspond to 10, 100, 1000 signal events after ten years of exposure. The red curves correspond to constant values of $c \tau_{\phi}$, the lifetime of $\phi$ times the speed of light. Middle: Hyper-K region (the three gray contours in the background are the same as those black ones in the upper panel) shown together with the existing constraints (from E949, NA62, CHARM, LSND, LHCb, Belle) and future reach by the upcoming experiments (ICARUS, SBND). Lower: The region of parameter space favored by the earlier KOTO excess is shown by the blue bands (dark and light blue correspond to 1 and $2 \sigma$ favored regions, respectively) [138-140]. The upper bound on the mixing angle $\theta$ derived from the latest KOTO analysis [141] is shown by the thick dark blue curve. The union of existing constraints excludes the gray shaded region. Like the upper and middle panels, the black curves corresponds to fixed number of signal events using Hyper-K to hunt atmospheric $\phi$ particles. For comparison, we also show the rescaled 10, 100, 1000 signal event contours (in pink color) for Super-K with 328 kiloton-year of data. In all the plots, the blue star corresponds to the benchmark point used in Eq. (6.11) and Fig. 6.4.

boundaries of the covered regions (enclosed by the black curves), the $\phi$ particles decay too fast to reach the detector and produce enough signal events, either due to large mixing angle $\theta$ or the opening of the $\phi \rightarrow \mu^{+} \mu^{-}$decay channel. The lower boundaries of the covered regions are simply set by the production rate which is proportional to $\theta^{2}$. In the same plot, the red contours correspond to constant values of $c \tau_{\phi}$, the lifetime of $\phi$ times the speed of light. They are not parallel to the upper edges of the black contours because $\phi$ particles are produced boosted.

Here, we only present contours for expected signal events. They indicate the region of parameter space that potentially could be covered with the Hyper-K detector. Once the backgrounds are fully understood, it is straightforward to derive an expected limit using our result. A thorough background analysis is beyond the scope of this chapter. Potentially important background includes atmospheric neutrinos undergoing neutral-current interaction with a $\pi^{0}$ radiation. The two photons from subsequent $\pi^{0}$ decay could also manifest as a double ring in water Cherenkov 
detectors. We can make an estimate of it based on a recent Super-Kamiokande analysis [160]. The number of $\pi^{0}$-like double ring events has been reported in Fig. 5 and Table II of [160]. Rescaling the result to Hyper-K, we expect to see a few thousand such background events. However, it is worth noting that the energy spectrum of the background $\pi^{0}$ peaks around $200 \mathrm{MeV}$ whereas the atmospheric $\phi$ energy peaks around $700 \mathrm{MeV}$ (see Fig. 6.1 above). This difference could serve a useful kinematical cut. In addition, a zenith angle distribution analysis (see Fig. 6.4 below) might be useful for further background discrimination.

In the middle panel of Fig. 6.3, we show the above found region of interest to Hyper-K together with the existing constraints, including the search for $K^{ \pm} \rightarrow$ $\pi^{ \pm} \phi \rightarrow \pi^{ \pm}+$invisible at E949 [146] (see also [142]) and the NA62 experiment [147149], displaced visibly-decaying $\phi$ search at CHARM $[142,150]$ and LSND [161], measurement of $B \rightarrow K \phi \rightarrow K \mu^{+} \mu^{-}$at LHCb $[162,163]$ and $B \rightarrow K \phi \rightarrow K+$ invisible at Belle [164] (see also [165]). We also show the future reach by the upcoming ICARUS, SBND experiments based on a recent analysis [156].

In the lower panel of Fig. 6.3, we zoom in toward the parameter space, where $m_{\phi} \in(100-200) \mathrm{MeV}$ and $\theta \sim($ a few $) \times 10^{-4}$, potentially relevant for KOTO. Again, the Hyper-K coverage is indicated by the thick black curves, with solid, dashed and dotted corresponding to observing 10, 100 and $1000 e^{+} e^{-}$pair events, respectively. Remarkably, they cover a new region of parameter space that has not been constrained before by any existing experiments. For comparison, we also show the rescaled 10, 100, 1000 signal event contours for the Super-Kamiokande experiment with 328 kilotonyear data collection.

Another attenuation effect before $\phi$ reaches the Hyper-K detector is the scattering with the earth. The energy of atmospheric $\phi$ is peaked around GeV scale, which roughly coincides with the nucleon mass and the QCD scale for strong inter- 
actions. The corresponding scattering cross section of $\phi$ with the nucleon target can be estimated to be, $\sigma_{\phi+N \rightarrow \pi^{0}+N} \sim g_{\pi N N}\left(\theta^{2} / m_{N}\right)^{2}\left(m_{N} / v\right)^{2} \sim 10^{-34} \theta^{2} \mathrm{~cm}^{2}$, where $v$ is the electroweak vaccum expectation value and $m_{N}$ is the nucleon mass. The extra suppression factor $\left(m_{N} / v\right)^{2}$ arises from the nucleon-Higgs coupling [166]. Given the earth nucleon density, $n \sim 10^{24} / \mathrm{cm}^{3}$, the free streaming length of $\phi$ is roughly, $l_{F}=1 /(n \sigma) \gtrsim 10^{5} \mathrm{~km} / \theta^{2}$. The free streaming length of $\phi$ through the earth is sufficiently long even for $\theta \sim \mathcal{O}(1)$.

Moreover, there is important information about the lifetime and mass of $\phi$ in the proposed signal, including the zenith angle and opening angle distributions of the final state $e^{+} e^{-}$pairs. In the top panel of Fig. 6.4, we plot the distribution of the zenith angle of $\phi$ particles arriving at the Hyper-K detector, for two sets of parameters. They exhibit very different behaviors, which can be understood by comparing the $\phi$ decay length, Eq. (6.11), and the distance it needs to travel before reaching the Hyper-K detector, $L(\varphi)$, given in Eq. (6.6). The first set of parameters, $m_{\phi}=150 \mathrm{MeV}, \theta=5 \times 10^{-4}$, corresponds to the blue star in Fig. 6.3. It represents an exciting and uncharted parameter space that will be explored by the upcoming intensity frontier experiments. In this case, $\gamma \beta \tau_{\phi} \sim 100 \mathrm{~km}$, for a typical boost factor (see Fig. 6.1), whereas $L(\varphi) \sim 10^{4}, 300,10 \mathrm{~km}$ for $\varphi=0, \pi / 2, \pi$, respectively. Clearly, if a $\phi$ particle travels to the detector from directions well below the horizon $(0<\varphi<\pi / 2)$, the distance $L(\varphi)$ is too long compared to $\gamma \beta \tau_{\phi}$ for it to survive. As a result, most of the $\phi$ particles are expected to arrive from above the Hyper-K detector's horizon $(\pi / 2<\varphi<\pi)$. For comparison, the second set of parameters has a much smaller $\theta$ leading to a much longer lived $\phi, \gamma \beta \tau_{\phi} \sim 10^{4} \mathrm{~km}$, thus $\phi$ could also arrive from directions below the horizon. However, smaller $\theta$ means fewer $\phi$ being produced from the atmosphere and such a point is beyond the reach of Hyper-K. Similarly, as $m_{\phi}$ increases beyond twice the muon mass, it mainly decays into $\mu^{+} \mu^{-}$, 

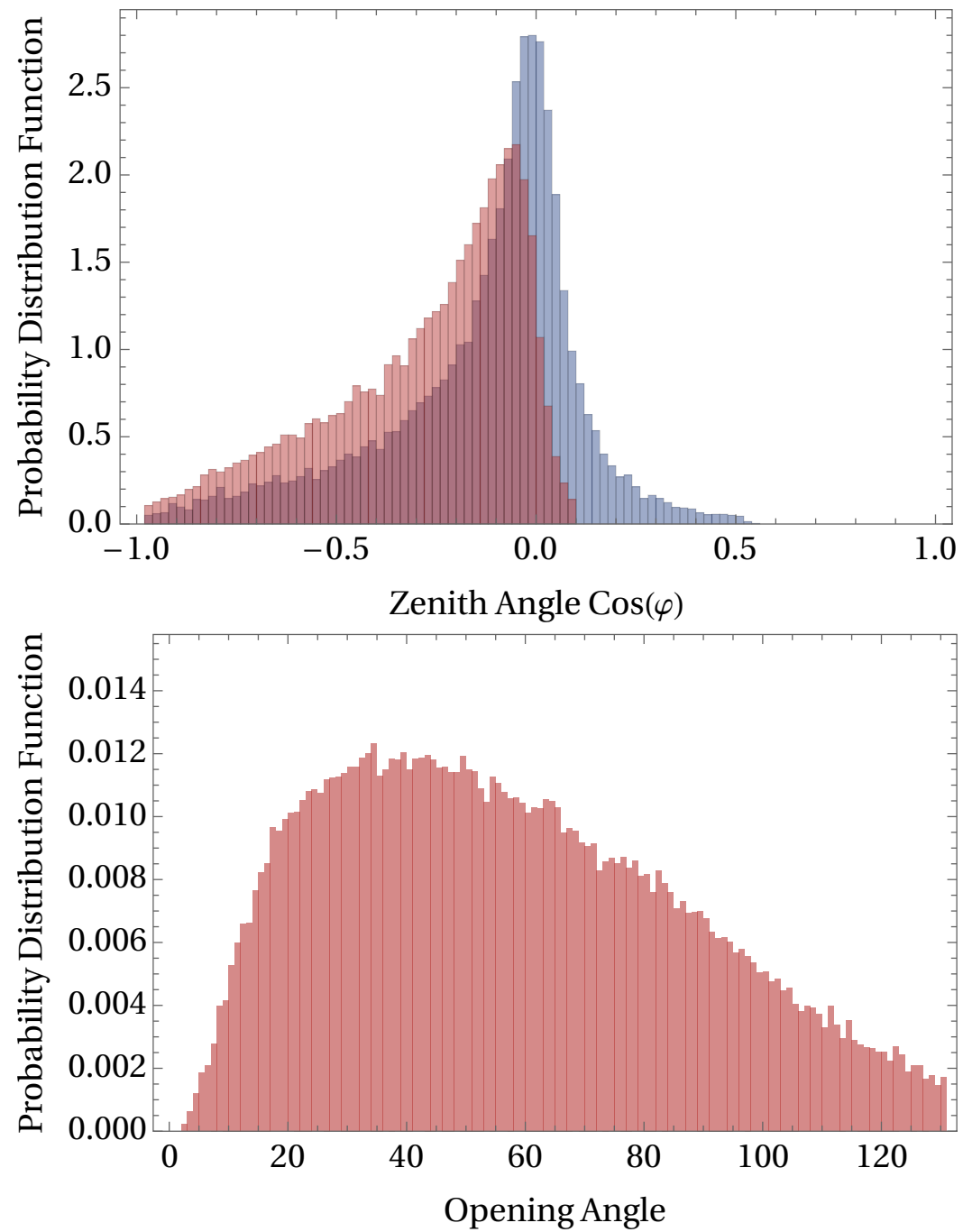

Figure 6.4: Additional kinematical features of the $\phi$ decay signal. Top: Zenith angle $\varphi$ distribution of the incoming into the Hyper-K detector for two sets of parameters, $m_{\phi}=150 \mathrm{MeV}, \theta=5 \times 10^{-4}$ (red) and $m_{\phi}=150 \mathrm{MeV}, \theta=10^{-4}$ (blue). The first point corresponds to the blue star in Fig. 6.3 and can leads to hundreds of $\phi$ decay events in Hyper-K. Most of the events are expected to arrive in directions above the detector's horizon. Bottom: electron-positron opening angle distribution from $\phi$ decay, for $m_{\phi}=150 \mathrm{MeV}$. The corresponding $\phi$ energy spectrum is shown in Fig. 6.1. 
via a much larger muon Yukawa coupling. The corresponding decay length is too short for $\phi$ to reach Hyper-K, unless $\theta$ is made much smaller, again resulting in a suppressed atmospheric production rate. In both latter cases, a larger detector would be needed.

In the bottom panel of Fig. 6.4, we plot the final state electron-positron opening angle distribution from $\phi$ decays, for $m_{\phi}=150 \mathrm{MeV}$. The result peaks around $\theta_{e^{+} e^{-}} \sim$ $30^{\circ}$, which is expected from the peak of $\phi$ energy distribution in Fig. 6.1, using $\theta_{e^{+} e^{-}} \sim 2 m_{\phi} / E_{\phi}$. A sizable fraction of events have a large $e^{+} e^{-}$opening angle. This quantity is relevant for the double ring signature to be resolved once they occur inside the Hyper-K detector. In the main plots, Fig. 6.3, we did not implement any cut on $\theta_{e^{+} e^{-}}$, which is straightforward to do once the threshold is established.

To summarize, we propose broadening the purpose of the Hyper-Kamiokande experiment though using it to hunt down long-lived Higgs portal scalar particles produced from the atmosphere. This proposal is in high complementarity to the intensity frontier experiments exploring rare meson decays. The target parameter space is for the scalar mass below twice the muon mass that is allowed by existing searches. The corresponding signal is electron-positron pair creations in the Hyper-K detector. We make approximations to the atmospheric production picture and derive a semi-analytical expression for the signal rate. In most events, the electron-positron opening angle is large enough for the double-ring signal to be resolved. If the doublerings are further used to reconstruct the decaying $\phi$ particles, one would find most of $\phi$ are arriving from directions above the detector's horizon. In the future, a more inclusive treatment of the $\phi$ production, better understanding of angular distribution measurement by the Hyper-K detector, as well as the background will be useful toward deriving a precise limit. The Hyper-K reach reported here for Higgs portal scalar similarly applies to light axion-like particles (of the DFSZ type $[167,168]$ ) which 
couple to Standard Model fermions also through their masses. The presence of small electron Yukawa coupling in the decay rates naturally makes these particles long lived and suitable to be searched for at earth-sized experiments.

It could be exciting to explore the proposed signal using the existing Super-K data. However, it is worth noting that the Super-K detector volume is about a factor of ten smaller than Hyper-K [169]. One could also consider searching for the signal at the future DUNE far detector which is made of liquid argon and is a few times smaller than Hyper-K in volume. This said, DUNE could be better at distinguishing $e^{ \pm}$from $\gamma$, which is useful for background discrimination. It is beyond the scope of this chapter to quantitatively compare the performance between Hyper-K and DUNE.

There have been recent proposals for further searches for long-lived light particles including the Higgs portal scalar at accelerator neutrino facilities using their near detectors $[156,161,170,171]$, as well as higher energy collider experiments with displaced detectors [54] (see the middle panel of Fig. 6.3). In comparison, the atmospheric $\phi$ particles carry relatively lower energies than their beam counterpart, thus the resulting $e^{+} e^{-}$opening angles are wider and easier for detection. Background is also much lower in the absence of a nearby intense beam. The very large Hyper-K detector volume partially compensates for the relatively lower atmospheric luminosity. All in all, there is excellent complementarity between the searches for long-lived particles of atmospheric and beam origins. 


\section{Chapter 7}

\section{On New Physics Contributions to the Higgs Decay to $Z \gamma$}

\subsection{Introduction}

The Higgs boson's interactions with the electroweak gauge bosons, $W, Z, \gamma$ are now well established. Decays to $W W^{*}[172-175], Z Z^{*}[176,177]$ and $\gamma \gamma[178]$ have all been measured and are consistent with Standard Model (SM) expectations at the $\mathcal{O}(20 \%)$ level. Production of the Higgs in association with a $W$ or $Z[173,174]$ is also consistent with the SM. One decay mode that, as of yet, has not been detected is $h \rightarrow Z \gamma$. The most recent searches for this channel from ATLAS [179] and CMS [180] place limits on the branching ratio relative to the SM prediction. The strongest limit comes from ATLAS which sets an upper upper bound on the $h \rightarrow Z \gamma$ signal strength at 3.6 times the Standard Model prediction.

The SM contribution to this decay is known at leading order (one-loop) [181,182] as well as higher order QCD corrections [183-185]. One-loop contributions from generic NP models are known [186] as well as contributions in SM effective field 
theory $[187,188]$. Phenomenological studies in specific models have also been carried out [189-202], as well as studies of this channel in the boosted regime [203], and explorations of how lepton colliders can probe the effective coupling of $h Z \gamma$ in Higgs production [204].

In this work we explore the space of new physics (NP) models that can give large contributions to $h \rightarrow Z \gamma$, close to the current experimental upper bound, without being in conflict with all other Higgs measurements. Due to precise measurements of the properties of the Higgs, and especially the $Z$ and $\gamma$, we assume these fields are SM-like and that the NP contribution to $h \rightarrow Z \gamma$ arises at one loop due to new particles that couple to the Higgs and have electroweak quantum numbers. The SM contributions to $h \rightarrow \gamma \gamma[205,206]$ and $h \rightarrow Z \gamma[181,182]$ are dominated by one-loop contributions (in contrast to $h \rightarrow Z Z$, which is dominated by tree-level contributions), so one-loop NP can make $\mathcal{O}(1)$ modifications to the rate. Even in this case, however, the strong constraints on $h \rightarrow \gamma \gamma$ make it difficult to engineer such models, and we show below that it is impossible to achieve this goal in models with a single new scalar multiplet. Therefore, if large deviations to $h \rightarrow Z \gamma$ are detected in the near future, this implies the existence of a complicated NP sector.

We will focus on NP scalars $\Phi$ with hypercharge and/or $S U(2)_{L}$ charge that contribute to $h \rightarrow Z \gamma$. The case of other spins for the intermediate particles will not produce qualitatively different results. For a scalar $\Phi$, regardless of its quantum numbers, one can always write renormalizable quartic interactions:

$$
\Phi^{\dagger} \Phi H^{\dagger} H,
$$

where $H$ is the SM Higgs doublet. ${ }^{1}$ These operators induce one-loop contributions

\footnotetext{
${ }^{1}$ We use $h$ to denote the physical Higgs scalar and $H$ to denote the scalar doublet
} 


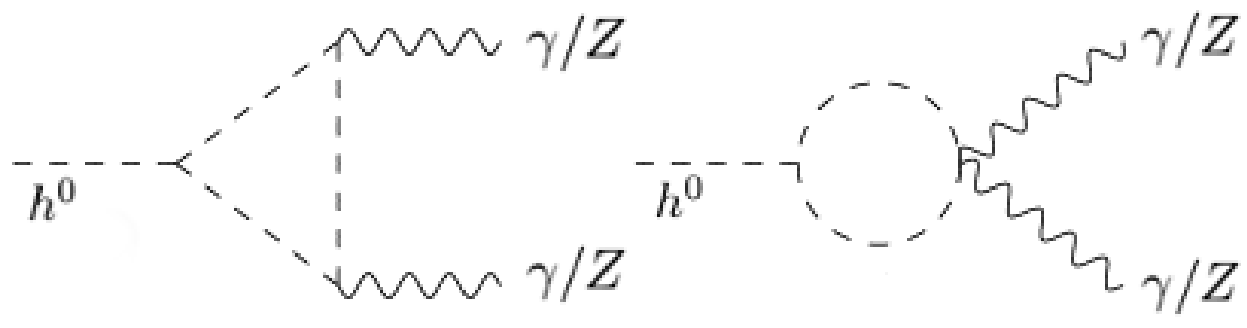

Figure 7.1: NP scalar one-loop contributions to Higgs to neutral diboson decays. The new scalar is represented by the unlabeled dashed lines.

to $h \rightarrow \gamma \gamma, h \rightarrow Z \gamma$, and $h \rightarrow Z Z$ of the type shown in Fig. 7.1. Trilinear operators written schematically as $H \Phi H$ are also possible for certain quantum numbers of $\Phi$, but these induce a vev for $\Phi$ which would give a significant contribution to the $\rho$ parameter $[22,207,208]$ that is excluded. With several multiplets with different quantum numbers, more complicated structures are possible: these will be discussed in Sec. 7.3.

New scalars with electroweak quantum numbers can be produced at hadron colliders such as the LHC and searched for directly, but the limits will depend strongly on their decay modes. The analysis here, however, is more model independent and only depends on the quantum numbers, the mass, and the coupling of the Higgs to the new states. At lepton colliders such as LEP, because of the relatively low background rates and fully known beam parameters, searches can be done in a more model independent manner [209-213]; these exclude masses below about $105 \mathrm{GeV}$. Given this lower limit, the Higgs cannot decay to an on-shell $\Phi$, so we can expand the amplitudes from the diagrams in Fig. 7.1 in powers of $m_{h}^{2} / 4 m_{\Phi}^{2}$. The leading term generated will be a $C P$-even dimension five operator:

$$
h F^{\mu \nu} F_{\mu \nu}
$$






Figure 7.2: Representation of the $h V V$ corrections to the $h \rightarrow 4 l$ amplitude where $V_{1,2}=Z, \gamma$ and $\ell, \ell^{\prime}=e, \mu$.

where $F$ is the field strength tensor of the either the $Z$ or $\gamma$.

Operators of the type given in Eq. (7.2) also contribute to the Higgs decay to four leptons, the so-called golden channel, via diagrams of the form shown in Fig. 7.2. The leading contribution to this decay is the tree-level contribution mediated by $Z Z^{*}$. Because this decay has four final state particles that can all be measured precisely, the rich final state kinematics can be used to gain significant information from each event. This means that one-loop contributions such as those from operators of the type in Eq. (7.2) can be probed [214-217] within the lifetime of the LHC. This has been used for various applications including the probing of exotic light states [218], measurement of the CP properties of the top Yukawa coupling [219], measurement of the ratio of the Higgs coupling to $W W$ relative to $Z Z$ [220], and the probing of various operators in SM effective field theory [221-223] as well as non-linear Higgs effects [224]. Even with the relatively small number of events already collected by the LHC, experiments can already use this data to place constraints on various scenarios [225].

Given models with large contributions to $h \rightarrow Z \gamma$, we can estimate how well those models can be probed in $h \rightarrow 4 \ell$ as a function of the number of events using 
the analysis techniques in $[217,226]$. We use a simple hypothesis testing procedure on a few benchmark points of these models and find that the high luminosity run of the LHC can reach approximately $2 \sigma$ sensitivity to these models in $h \rightarrow 4 \ell$.

Rather than adding arbitrary new scalar multiplets, one can also ask if well motivated models can produce significant deviations in $h \rightarrow Z \gamma$. Supersymmetric models are extremely well studied [10], and they contain scalar partners of the top quark, stops, which have large couplings to the Higgs and carry electroweak charges, so they can be scalars of the type shown in Fig. 7.1. The stops carry colour and, as such, contribute to production of the Higgs via gluon fusion in addition to the Higgs to diboson decays — measurements of these processes (especially $h \rightarrow \gamma \gamma$ ) can be used to place constraints on stop parameter space [227]. Here we update the constraints from [227] and show that these constraints imply that stops can only make small modifications to $h \rightarrow Z \gamma$. We also find that $h \rightarrow 4 \ell$ at the LHC will not be sensitive to these models, but there may be sensitivity at future higher energy hadron colliders.

Another well motivated model is folded SUSY [228], which also contains scalar top partners, F-stops, that have electroweak quantum numbers but do not carry colour. This makes direct bounds much weaker, and it also eliminates constraints from Higgs production via gluon fusion, making indirect bounds also weaker [227]. We also update the analysis on F-stops. While their contributions to $h \rightarrow Z \gamma$ can be larger than for ordinary stops, it still cannot be large, and the conclusions for the four lepton analysis is similar to that of stops.

The rest of this chapter is organized as follows: Sec. 7.2 establishes the conventions used to describe new physics and the procedure used in analyzing the four-lepton processes, and Sec. 7.3 presents the construction and signals of models with large contributions to $h \rightarrow Z \gamma$. Sec. 7.4 presents an analysis of supersymmetric models and 
Sec. 7.5 wraps everything up.

\subsection{Higgs Physics}

Measurements of production and decay rates of the Higgs are all consistent with SM predictions. Therefore if there is new physics with electroweak charge that couples to the Higgs, it will be constrained by its contributions to the decay of Higgs to $\gamma \gamma$ at one loop. Since the leading SM contribution is also at one-loop, the constraints on such new physics at the weak scale will be strong. Similarly, new physics with colour charge that couples to the Higgs will contribute to the Higgs production via gluon fusion and can also place strong constraints.

Assuming the Higgs width is small compared to its mass, the cross section of a specific production mode $i$ and decay to a specific final state $V V^{\prime}$ can be parameterized as [229]:

$$
\sigma\left(i \rightarrow h \rightarrow V V^{\prime}\right)=\frac{\sigma_{i}(\vec{\kappa}) \Gamma^{V V^{\prime}}(\vec{\kappa})}{\Gamma_{H}}
$$

Where $\Gamma_{H}$ is the total decay width of the Higgs, $\Gamma^{V V^{\prime}}$ is the partial decay width to $V V^{\prime}$, and $\sigma_{i}$ is the total cross section of ( $i \rightarrow h \rightarrow$ Anything) in the $i$ production mode. Deviations from the SM can be parameterized through a set of coupling strength modifiers $\vec{\kappa}[178]$. For a given production process or decay mode $j$, these are defined by:

$$
\kappa_{j}^{2}=\frac{\sigma_{j}}{\sigma_{j, S M}} \quad \text { or } \quad \kappa_{j}^{2}=\frac{\Gamma^{j}}{\Gamma_{S M}^{j}} .
$$

The SM has all $\kappa_{j}$ values equal to unity. The $\kappa$ framework is not a full theory, and there are effects that it cannot account for that are detailed in [229]. The largest such effects are in processes where the Higgs is off-shell and not relevant for our analysis. We leave a more detailed accounting of the error budget due to this framework to 
future work.

Generic NP contributions can be constrained by the limits placed by experimental measurements on Higgs boson coupling strength modifiers, $\kappa_{j}$. In the models we are considering where the new physics dominantly contributes at one loop, the relevant coupling modifiers are those to photons, gluons, and $h \rightarrow Z \gamma: \kappa_{\gamma}$ and $\kappa_{g}$, and $\kappa_{Z \gamma}$ respectively. The kinematics of the measured processes do not change significantly in the presence of new physics above half the Higgs mass [178], so the $\kappa$ framework is sufficient to describe deviations from the SM.

In Fig. 7.3 we show the constraints in the $\kappa_{\gamma}-\kappa_{g}$ plane from [178] assuming all other couplings are SM-like. The best fit values resulting from their analysis are $\kappa_{\gamma}=1.16_{-0.14}^{+0.14}$ and $\kappa_{g}=0.76_{-0.14}^{+0.17}$. On the other hand, the upper bounds on the Higgs decay to $Z \gamma$ are, at $95 \%$ confidence level, 3.6 times the Standard Model prediction for the production cross-section times the branching ratio for $p p \rightarrow h \rightarrow Z \gamma-$ this drops slightly to 3.57 times the SM prediction if the Higgs boson production is set to the SM value. The best fit value for the signal yield is $2.0_{-0.9}^{+1.0}$ [179], normalized to the SM. The future HL-LHC is expected to improve these constraints for the $p p \rightarrow h \rightarrow Z \gamma$ process to $1.00 \pm 0.23$ the SM prediction [230]. In this work we will explore models that are not excluded by the $\kappa_{\gamma}-\kappa_{g}$ analysis but that can have large contributions to $h \rightarrow Z \gamma$, possibly saturating the current experimental constraint of $\sim 3.5$ times the SM prediction.

\subsection{1 $h \rightarrow 4 \ell$ at One Loop}

Despite the small rate, the $h \rightarrow 4 \ell$ mode benefits from a rich kinematic structure and very high signal to background ratio. This, coupled with systematic uncertainties that are very different (and typically smaller) than with direct diboson measurements, make the four-lepton channel an important complementary way to examine the Higgs 


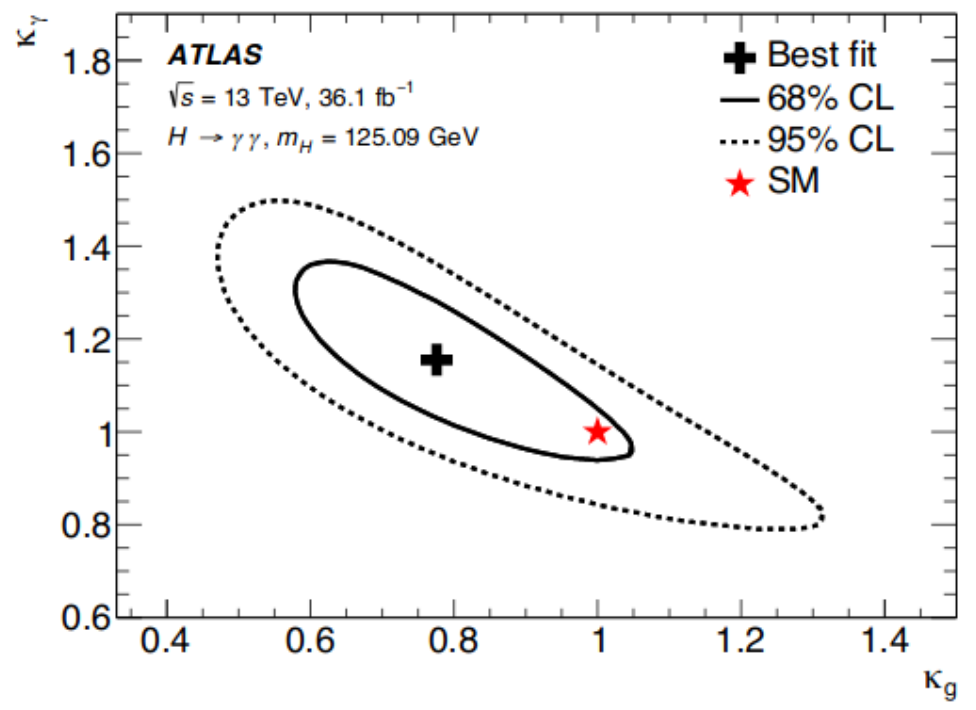

Figure 7.3: Likelihood contours in the $\kappa_{\gamma}-\kappa_{g}$ plane from ATLAS data [178]. All other coupling modifiers are fixed to the SM values.

sector. Here, we follow the analysis of $[217,226]$ in order to constrain these models using their contribution to $h \rightarrow 4 \ell$ at one loop. The dominant contribution to $h \rightarrow$ $4 \ell$ is via virtual gauge bosons with generic diagrams of the form of Fig. 7.2. The contributions to the $h V V$ couplings can be described by an effective Lagrangian:

$$
\mathcal{L}=\mathcal{L}_{0}+\mathcal{L}_{1}+\ldots
$$

Here, the leading order term that contributes to the $h Z Z$ coupling at tree level is given in $\mathcal{L}_{0}$. This term gifts the $Z$ particle its mass and is generated via EWSB,

$$
\mathcal{L}_{0}=\frac{h}{2 v} A_{1}^{Z Z} m_{Z}^{2} Z^{\mu} Z_{\mu}
$$

where $Z_{\mu}$ is the $Z$ boson field. For the SM at tree level, we have $A_{1}^{Z Z}=2$. Additionally, there are dimension-5 operators that can parameterize the one-loop contri- 
butions:

$$
\mathcal{L}_{1}=\frac{h}{4 v}\left(A_{2}^{Z \gamma} F^{\mu \nu} Z_{\mu \nu}+A_{2}^{\gamma \gamma} F^{\mu \nu} F_{\mu \nu}+A_{2}^{Z Z} Z^{\mu \nu} Z_{\mu \nu}\right)
$$

where $Z_{\mu \nu}\left(F_{\mu \nu}\right)$ is the field strength of the $Z$ (photon). These Lagrangian terms are only a subset of a more general description that would include other dimension five operators, but those operators are constrained to be too small to be relevant for this analysis [219,223]. Moving forward, we neglect the contributions of $A_{2}^{Z Z}$ due to the lack of sensitivity $[217,226,231]$ of future measurements to such modifications.

It should also be noted that in the SM there are one-loop box and pentagon diagrams that contribute to the $h \rightarrow 4 \ell$ process that are not captured by the parameterization of Eq. 7.7. These contributions are small, relative to the tree-level process, and are unaffected by the presence of any of the BSM models presented here. These calculations have been done [232,233], and we leave the integration of these results into our framework for future work. As such, we now turn our attention to the most relevant operators in the search for BSM: $A_{2}^{\gamma \gamma}$ and $A_{2}^{Z \gamma}$.

Within the SM the $A_{2}^{V \gamma}$ couplings will be generated primarily via a $\mathrm{W}$ boson loop along with (smaller) contributions from a top loop. Numerically, the SM values are $A_{2}^{\gamma \gamma} \approx-0.008$ and $A_{2}^{Z \gamma} \approx-0.014[220] .^{2}$ These form factors can be used to compute on-shell Higgs decay to $\gamma \gamma$ or $Z \gamma$. These SM one-loop contributions have been explored in the literature for both $h \rightarrow Z \gamma[181,182]$ and $h \rightarrow \gamma \gamma[205,206]$. We have assumed NP contributions are $C P$-even because of strong constraints from flavour and $C P$ violating observables $[235] .^{3}$

Given this parameterization, we express the matrix element for the $h \rightarrow 4 l$

\footnotetext{
${ }^{2}$ Note that [234] gives a different sign for $A_{2}^{Z \gamma}$.

${ }^{3}$ For analyses of $C P$ violation in $h \rightarrow Z \gamma$ see [236-238].
} 
process in the form,

$$
\begin{aligned}
& \mathcal{M}(h \rightarrow 4 l)=\mathcal{M}^{\mu \nu}\left(h \rightarrow V_{1} V_{2}\right) \times \\
& \mathcal{P}_{\mu \alpha}\left(V_{1}\right) \mathcal{M}^{\alpha}\left(V_{1} \rightarrow 2 l\right) \mathcal{P}_{\nu \beta}\left(V_{2}\right) \mathcal{M}^{\beta}\left(V_{2} \rightarrow 2 l\right),
\end{aligned}
$$

where $V=Z, \gamma$, and $\mathcal{P}_{\mu \nu}\left(V_{i}\right)$ are the propagators of the vector bosons. It should be noted that the first line is where any potential NP that we could hope to uncover would reside. The second line is simply vector bosons propagating and decaying to leptons - processes that are well understood and well measured. The $h \rightarrow V_{1} V_{2}$ matrix element can be parameterized as follows:

$$
\begin{aligned}
& \mathcal{M}^{\mu \nu}\left(h \rightarrow V_{1} V_{2}\right)=\frac{1}{v} C_{1}^{i} m_{z}^{2} g^{\mu \nu}+ \\
& \frac{1}{v} C_{2}^{i}\left(k_{1}^{\nu} k_{2}^{\mu}-k_{1} \cdot k_{2} g^{\mu \nu}\right)+\frac{1}{v} C_{3}^{i} \epsilon^{\mu \nu \alpha \beta} k_{1 \alpha} k_{2 \beta},
\end{aligned}
$$

with $i=Z Z, Z \gamma, \gamma \gamma$ and $k_{1}, k_{2}$ representing the four momenta of the intermediate vector bosons (or, equivalently, lepton pairs).

The form factors, $C_{n}^{i}$, are Lorentz invariant and encode the momentum dependence. They have the form,

$$
C_{n}^{i} \sim h_{X} f_{i}\left(m_{h}^{2} / m_{X}^{2}, k_{1}^{2}, k_{2}^{2}\right)
$$

with $f_{i}\left(m_{h}^{2} / m_{X}^{2}, k_{1}^{2}, k_{2}^{2}\right)$ representing the loop function for NP particle $X$ coupled to the Higgs with coupling $h_{X}$. Previous studies have indicated that dependence on the invariant mass parameters $k_{i}^{2}$ is quite weak [219,239]. In order to examine this claim, the form factors $C_{n}^{i}$ were expressed as a Taylor Series and expanded around the pole masses of the intermediate vector bosons. The parameter space examined here can indeed be probed taking $k_{i}^{2}=m^{2}$ for the relevant boson, i.e. treating the vector as 
on-shell for the form factor. This limit is used for determining the exclusion bounds from ATLAS measurements of Higgs to diphotons.

All the NP scenarios we will explore can then be encoded in the value of the effective field theory (EFT) operators $A_{2}^{\gamma \gamma}$ and $A_{2}^{Z \gamma}$ for all models (plus the gluon form factor $A_{2}^{g g}$ for coloured new scalars such as stops). They are computed via the diagrams in Fig. 7.1 assuming the intermediate state gauge bosons are on-shell - in the language of Eq. 7.9 this makes $A_{2}^{\gamma \gamma}=2 C_{2}^{\gamma \gamma}$ and $A_{2}^{\gamma \gamma}=2 C_{2}^{Z \gamma}$.

\subsubsection{Kinematic Analysis of $h \rightarrow 4 \ell$}

In the Higgs rest frame, the useful kinematic variables for this decay are:

- $\Phi$ : The decay angle between the decay planes of the intermediate bosons in the rest frame of the Higgs.

- $\theta_{1}$ : The angle between the lepton coming from the decay of $V_{1}$ and the momentum of $V_{2}$ in the $V_{1}$ rest frame.

- $\theta_{2}$ : Identical to $\theta_{1}$ but with $V_{1}$ and $V_{2}$ swapped.

- $M_{i}$ : The invariant mass of the lepton pair produced by the decay of the $i^{t h}$ boson. By convention $M_{1}>M_{2}$.

In the case of intermediate $Z \gamma$ vectors, the rate is dominated by $M_{1} \approx M_{Z}$. Kinematics require $M_{1}+M_{2} \leq \sqrt{s}$ where $s$ is the invariant mass squared of the four lepton system. We refer to the set of these variables for a single $4 \ell$ event as $Y-$ this will be used as the input for the likelihood analysis to test between the Standard Model and different NP scenarios, or equivalently as ways to test different values of the form factors $A_{2}^{V V^{\prime}}$. For different values of the form factors, we generate Monte 
Carlo (MC) events for the process $g g \rightarrow h \rightarrow 4 l$ at the $14 \mathrm{TeV}$ LHC using MadGraph5_aMC@NLO [240]. Since gluon fusion is the primary production mechanism for the Higgs at the LHC [241] and the variables examined during our analysis are in the rest frame of the Higgs and thus only sensitive to the decay process, including other production modes would not change our analysis. Additionally, the very high signal to background ratio of the four lepton channel and the easy identification of leptons within LHC detectors makes both a full simulation of backgrounds and an examination of detector effects subleading for the purposes of this work [231]. Measurements (see for example [242]) in this channel confirm that the signal to background ratio is very large in the Higgs invariant mass window.

We now determine the number of events needed to distinguish two different hypotheses (SM vs. NP) at a given confidence level. This is done with a likelihood analysis of the MC generated NP events compared to MC events assuming purely SM physics. A standard unbinned likelihood analysis is used (see $[215,243]$ for further details) where the computed differential cross-section can be normalized and used as a probability over the $4 l$ kinematic variables:

$$
P(Y \mid A)=\frac{|\mathcal{M}(Y, A)|^{2}}{\int_{\text {fid }}|\mathcal{M}(Y, A)|^{2} \mathrm{dY}},
$$

where $Y$ is the set of kinematic variables of an event, $A$ is the given set of effective coupling strengths for the model being examined, and $\mathcal{M}$ is the matrix element from Eq. 7.8. The integral is over the fiducial acceptance region for $4 \ell$ events. If we have $N$ events, this allows us to compute a likelihood: $\mathcal{L}(A)=\prod_{i=1}^{N} P\left(Y_{i} \mid A\right)$. If we have two different scenarios (typically a SM scenario and a NP scenario), we can calculate 
a likelihood ratio and construct a hypothesis test statistic,

$$
\Lambda=2 \log \left(\mathcal{L}\left(A_{1}\right) / \mathcal{L}\left(A_{2}\right)\right)
$$

This test statistic is calculated on MC data generated assuming each of the two underlying hypotheses. This creates two distributions of the test statistic $\Lambda$, one assuming scenario 1 is true, the other, scenario 2. The separation between these distributions is a measure of how easily distinguishable the two hypotheses are. Explicitly, if we call the distribution with the smaller average test statistic $f$ and the other distribution $g$ then there exists a value $\Lambda_{0}$ such that,

$$
\int_{-\infty}^{\Lambda_{0}} f(\Lambda) \mathrm{d} \Lambda=\int_{\Lambda_{0}}^{\infty} g(\Lambda) \mathrm{d} \Lambda
$$

Because $\Lambda_{0}$ is the point at which the distributions are indistinguishable, the value on either side of Eq. 7.13 is the one-sided Gaussian probability of the observed events of one scenario excluding the alternative. This can be converted into a more standard $\sigma$ value that is a function of the number of signal events $N$. In other words, we can get the expected statistical significance as a function of the number of events for any two scenarios.

\subsection{Models with large $h \rightarrow Z \gamma$ contributions}

Here, we explore models with new electroweak multiplets that can give large contributions to $h \rightarrow Z \gamma$ while not being in conflict with $h \rightarrow \gamma \gamma$. 


\subsubsection{New Scalar Multiplets}

We begin with a simple scenario: a single scalar multiplet that is charged under $\mathrm{SU}(2) \times \mathrm{U}(1)$, but is a singlet under $\mathrm{SU}(3)_{c}$. At the moment, we remain agnostic to the size of the multiplet; we do note, however, that the size of the multiplet is bounded at 8 (isospin of $7 / 2$ ) due to perturbative unitary constraints [244]. Finally, in order to stay away from any LEP II bounds [209-213], we require our particles have a mass $\gtrsim 100 \mathrm{GeV}$.

The NP contributions to our effective operators $A_{2}^{Z \gamma}$ and $A_{2}^{\gamma \gamma}$ have the form

$$
\begin{array}{r}
A_{2}^{\gamma \gamma}=\sum_{\text {particles }} 2 h_{X} g_{X}^{2} * l_{\gamma \gamma}[m] \\
A_{2}^{Z \gamma}=\sum_{\text {particles }} 2 h_{X} g_{X} z_{X} * l_{Z \gamma}[m]
\end{array}
$$

where $h_{X}$ is the coupling of the new particles to the Higgs, $g_{X}$ and $z_{X}$ are the coupling of the new particles to photons and $Z$ bosons, respectively, and $l_{\gamma \gamma}$ and $l_{Z \gamma}$ are the loop functions that depend on the mass of the NP particles given in App. B. The sum is over all states in the multiplet. It should be noted that, unless there is mass mixing, $h_{X}$ and the particle mass is the same for all members of the multiplet. The couplings $g_{X}$ and $z_{X}$, on the other hand, depend on the $T_{3}$ value for the particle and are thus different for every multiplet member. Additionally, for NP particles with

mass $\gtrsim 100 \mathrm{GeV}$, the loop functions become approximately equal, $l_{\gamma \gamma}[m] \approx l_{Z \gamma}[m]$, so the relative contribution sizes of the new particle multiplet to the diphoton vs. $Z \gamma$ is controlled by the relative size of

$$
\begin{aligned}
& g_{\gamma \gamma}^{\mathrm{eff}}=\sum g_{X}^{2} \\
& g_{Z \gamma}^{\mathrm{eff}}=\sum g_{X} z_{X},
\end{aligned}
$$


with the sum being over all particles in the multiplet.

The relevant terms in the Lagrangian for a single scalar multiplet $X$ are given by

$$
\mathcal{L} \supset\left(D_{\mu} X\right)^{\dagger}\left(D^{\mu} X\right)+m_{X}^{2} X^{\dagger} X+b\left(X^{\dagger} X\right)\left(H^{\dagger} H\right)
$$

with $D_{\mu}$ being the typical covariant derivative that gives rise to the coupling of $X$ to gauge bosons. The coupling $b$ gives rise to the Higgs coupling to $X$ pairs after electroweak symmetry breaking, and we ignore $X$ self-interactions as they do not affect the analysis. We assume $m_{X}^{2}>0$, as bounds on new electroweak scalars with vacuum expectation values are very strong [22,207,208] (unless $X$ has the same quantum numbers as the Higgs). For the same reason, we do not include $H X H$ terms which are allowed for certain quantum numbers of $X$. Finally, we remain agnostic as to which decay modes are present for the new particles; this allows us to ignore direct search limits and focus wholly on Higgs decays.

After a rotation into the mass basis for the gauge bosons, the aforementioned couplings for the new scalars are:

$$
\begin{gathered}
g_{X}=g T_{3} s_{W}+g^{\prime} Y c_{W} \\
z_{X}=g T_{3} c_{W}-g^{\prime} Y s_{W},
\end{gathered}
$$

where $g$ and $g^{\prime}$ are the gauge couplings for $S U(2)_{L} \times U(1)_{Y}$ and $s_{W}$ and $c_{W}$ are the sine and cosine of the weak mixing angle. The typical convention relating electric charge of the scalars to the isospin and hypercharge holds: $Q=T_{3}+Y$. Requiring integer charge and half-integer (integer) values for weak-isospin restrict hypercharge to half-integer (integer) values. 


\subsubsection{The Failure Of One Additional Multiplet}

We now demonstrate that the inclusion of a single multiplet cannot give rise to large contributions to $h \rightarrow Z \gamma$. The relevant products of couplings to gauge bosons are given by

$$
\begin{aligned}
g_{X}^{2} & =g^{2} T_{3}^{2} s_{W}^{2}+2 g g^{\prime} T_{3} c_{W} s_{W} Y+g^{\prime 2} c_{W}^{2} Y^{2} \\
g_{X} z_{X} & =g^{2} T_{3}^{2} s_{W} c_{W}+g g^{\prime} T_{3}\left(c_{W}^{2}-s_{W}^{2}\right) Y-g^{\prime 2} c_{W} s_{W} Y^{2} .
\end{aligned}
$$

Summing over $T_{3}$ gives effective couplings (defined in Eq. (7.15))

$$
\begin{aligned}
& g_{\gamma \gamma}^{e f f}=K *\left(g^{2} \sin ^{2} \theta_{W}\right)+k *\left(g^{\prime 2} \cos ^{2} \theta_{W} Y^{2}\right) \\
& g_{Z \gamma}^{e f f}=K *\left(g^{2} \sin \theta_{W} \cos \theta_{W}\right)-k *\left(g^{\prime 2} \cos \theta_{W} \sin \theta_{W} Y^{2}\right)
\end{aligned}
$$

where $k$ is the size of the multiplet and

$$
\begin{aligned}
& K=2 \sum_{i}^{i=\frac{k-1}{2}} i^{2} k \text { odd } \\
& K=\frac{1}{2} \sum_{i}^{i=\frac{k}{2}} i^{2} k \text { even. }
\end{aligned}
$$

Since $g^{\prime 2} \cos ^{2} \theta_{W}>g^{\prime 2} \cos \theta_{W} \sin \theta_{W}, g_{\gamma \gamma}^{e f f}$ will always increase in strength faster than $g_{Z \gamma}^{e f f}$ as the absolute value of the multiplet's hypercharge becomes larger. So, to maximize the relative size of the $Z \gamma$ contributions, the magnitude of the multiplet's hypercharge must be kept as small as possible. Therefore, the best case scenario is an odd multiplet with $Y=0$ that gives

$$
\frac{g_{Z \gamma}^{e f f}}{g_{\gamma \gamma}^{e f f}}=\cot \theta_{W} \approx 1.86
$$


The even multiplets give weaker results, though as $k$ becomes larger, this ratio approaches the same value as the odd multiplets. When we consider the fact that in the SM the $Z \gamma$ effective operator is larger than the $\gamma \gamma$ one,

$$
\left.\frac{A_{2}^{Z \gamma}}{A_{2}^{\gamma \gamma}}\right|_{\mathrm{SM}} \approx 1.75
$$

this shows the modification of the $h \rightarrow Z \gamma$ and $h \rightarrow \gamma \gamma$ rates are at best comparable. As such, the large experimentally allowed $h \rightarrow Z \gamma$ parameter space cannot be populated by such simple models and we must add another degree of complexity through the addition of more multiplets. These scenarios can result in large $h \rightarrow Z \gamma$ rates through the cancellation of $h \rightarrow \gamma \gamma$ contributions and/or with mass mixing effects such that the mass eigenstates with comparatively small $\gamma$ couplings have low masses and those with larger electric charges have larger masses. The mixing scenario turns out to be less compelling: even if one were to obtain mass mixing such that the member of the multiplet with the largest $T_{3}$ value (e.g. $+1 / 2$ for a doublet) that has the largest contribution to $h \rightarrow \gamma \gamma$ relative to $h \rightarrow Z \gamma$ is effectively decoupled, the improvements to Eq. (7.21) are minor. Even with more complicated scenarios, it is extremely difficult to construct a model with a viable scalar potential that achieves the required mass splitting. With this in mind, for the rest of this section we explore cancellation scenarios.

\subsubsection{The Singlet-Triplet Model}

The road map of where to go next comes from analyzing this question from the point of view of the unbroken electroweak effective field theory. In the EFT prior to spontaneous symmetry breaking [245-247] (typically called SMEFT) there are three 
dimension-6 terms relevant to Higgs to electroweak diboson decays [248]:

$$
\begin{aligned}
& \text { - } Q_{W} H^{\dagger} H W^{\mu \nu a} W_{\mu \nu}^{a} \\
& \text { - } Q_{B} H^{\dagger} H B^{\mu \nu} B_{\mu \nu} \\
& \text { - } Q_{W B} H^{\dagger} \tau^{a} H B^{\mu \nu} W_{\mu \nu}^{a}
\end{aligned}
$$

where $W^{\mu \nu a}$ and $B^{\mu \nu}$ are the field strength tensors of $S U(2)_{L}$ and $U(1)_{Y}$ respectively, and $\tau^{a}$ are Pauli matrices. The introduction of scalar multiplets can generate contributions to the Wilson coefficients $Q$ of either sign: this can lead to multiplets that either enhance, dampen, or even nullify each other's effects.

We can get expressions for our dimension-5 EFT in the Higgs basis defined in Eq. (7.7) in terms of our unbroken operators:

$$
\begin{array}{r}
A_{2}^{Z \gamma}=4 v^{2}\left(s_{W} c_{W}\left(Q_{W}-Q_{B}\right)+\left(s_{W}^{2}-c_{W}^{2}\right) Q_{W B}\right) \\
A_{2}^{\gamma \gamma}=8 v^{2}\left(s_{W}^{2} Q_{W}+c_{W}^{2} Q_{B}-2 s_{W} c_{W} Q_{W B}\right) .
\end{array}
$$

In the 3-dimensional space of $Q_{W}, Q_{B}$, and $Q_{W B}$, there is a plane of values that lead to no contribution to $A_{2}^{\gamma \gamma}$ - yet the vast majority of this plane (everything outside one line) does offer up contributions to $A_{2}^{Z \gamma}$. The task, then, becomes finding an appropriate set of multiplets that can simultaneously enhance the rate of $h \rightarrow Z \gamma$ and leave $h \rightarrow \gamma \gamma$ close to its SM value.

A model that features these properties is one where we introduce two NP scalar multiplets: a singlet state, $S$, with $Y=1$ and a triplet state, $T$, with $Y=0$. Both multiplets are singlets under $\mathrm{SU}(3)_{c}$. At leading order, integrating out $S$ generates $Q_{B}$ and integrating out $T$ gives $Q_{W}$. Therefore, by choosing couplings appropriately, we can be on the line in the $Q_{B}-Q_{W}$ plane where the contributions to $h \rightarrow \gamma \gamma$ (approximately) vanish. As we will consider relatively light states, we will use the EFT 
as only a guide and do our analysis in the full theory.

The relevant NP Lagrangian terms are:

$$
\begin{array}{r}
\mathcal{L} \supset\left(D_{\mu} S\right)^{\dagger}\left(D^{\mu} S\right)+\left(D_{\mu} T\right)^{\dagger}\left(D^{\mu} T\right)+m_{S^{2}} S^{\dagger} S \\
+m_{T}^{2} T^{\dagger} T+a\left(S^{\dagger} S\right)^{2}+b\left(S^{\dagger} S\right)\left(H^{\dagger} H\right) \\
+c\left(T^{\dagger} T\right)^{2}+d\left(T^{\dagger} T\right)\left(H^{\dagger} H\right)+e\left(S^{\dagger} S\right)\left(T^{\dagger} T\right) .
\end{array}
$$

The contributions to the Higgs to diboson decays are simply those from Eq. (7.14) generalized to allow for multiple particles,

$$
\begin{array}{r}
A_{2}^{\gamma \gamma}=2\left(h_{S} g_{S}^{2} * l_{\gamma \gamma}\left[m_{S}\right]+h_{T} g_{T \gamma \gamma}^{e f f} * l_{\gamma \gamma}\left[m_{T}\right]\right) \\
A_{2}^{Z \gamma}=2\left(h_{S} g_{S} z_{S} * l_{Z \gamma}\left[m_{S}\right]+h_{T} g_{T Z \gamma}^{e f f} * l_{Z \gamma}\left[m_{T}\right]\right),
\end{array}
$$

where $h_{S}$ and $h_{T}$ are equal to $b v$ and $d v$, respectively; while $g_{T \gamma \gamma}^{e f f}$ and $g_{T Z \gamma}^{\text {eff }}$ are calculated through Eq. (7.19). Just as in the single NP multiplet case, the particle masses we consider are greater than $100 \mathrm{GeV}$ and, as a result, the loop functions for $\gamma \gamma$ and $Z \gamma$ are nearly identical.

Cancellation of the NP contributions to $A_{2}^{\gamma \gamma}$ requires either $h_{S}$ or $h_{T}$ (or, in terms of the Lagrangian, $b$ or $d$ ) to be negative. For concreteness we select $h_{S}$ to be negative. The scalar potential must be bounded from below in order to maintain its stability: this can be checked by ensuring that the quartic terms of the scalar potential have a limit $V_{s} \geq 0$ in all directions (any direction where $V_{s}=0$ also requires the limit of the quadratic terms in that direction to be $\geq 0$ ). This requires the quartic couplings $a$ and $c$ to be positive, and we have also taken $d>0$. As it does not affect our analysis, we can also take $e>0$.

The only remaining constraint is $b<0$ potentially leading to an unbounded direction. This analysis is nearly identical to those in 2 Higgs Doublet Models [249]. 
Setting $H^{\dagger} H=r \cos \theta$ and $S^{\dagger} S=r \sin \theta$, followed by taking the large $r$ limit gives

$$
V=r^{2}\left(a \sin ^{2} \theta+\lambda \cos ^{2} \theta+b \cos \theta \sin \theta\right)=r^{2} f(\theta)
$$

where $\lambda$ is the SM quartic Higgs coupling. Ensuring Eq. (7.27) is positive can be done by requiring $f(\theta) \geq 0$ at its smallest point. Applying this requirement enforces a minimum size on the negative coupling $b$ :

$$
b \geq-\sqrt{4 a \lambda}
$$

The singlet-triplet model is representative of a much larger class of possible models - not a unique solution to a large $Z \gamma$ signal. This construction can apply to two multiplets of any sizes, although this particular choice of charges is simpler than the generic one because there are no allowed mass mixing terms.

Within this model, we will work with the following benchmark parameter point:

$$
\begin{aligned}
& \text { - } m_{S}=105 \mathrm{GeV} \\
& \text { - } m_{T}=140 \mathrm{GeV} \\
& \text { - } a=1.6 \\
& \text { - } b=-0.9 .
\end{aligned}
$$

This point evades constraints from LEP [209-213], has reasonable coupling sizes, scalar potential stability, and is within the $2 \sigma$ limits on $h \rightarrow \gamma \gamma$ [178]. Full cancellation of the NP diphoton decay contributions is possible - however to avoid tuning parameters we are content with values that respect current bounds. This point also saturates the ATLAS [179] upper limit on $h \rightarrow Z \gamma$. We take this as a representative of the relatively complicated models required to generate a large anomaly in 
$h \rightarrow Z \gamma$ while being consistent with other constraints.

\subsubsection{Four-Lepton Sensitivity}

Here we calculate the number of $h \rightarrow 4 \ell$ events required to probe the above benchmark point in the singlet-triplet model using the analysis described in section 7.2.2. From the loop diagrams in Fig. 7.1, we can determine the contributions to the effective operators $A_{2}^{\gamma \gamma}$ and $A_{2}^{Z \gamma}$ :

$$
\begin{aligned}
& \frac{A_{2}^{\gamma \gamma}(\mathrm{NP})}{A_{2}^{\gamma \gamma}(\mathrm{SM})}=\frac{-0.00122}{-0.008}=0.1525 \\
& \frac{A_{2}^{Z \gamma}(\mathrm{NP})}{A_{2}^{Z \gamma}(\mathrm{SM})}=\frac{-0.00855}{-0.014}=0.6107
\end{aligned}
$$

The modification of the branching ratio (or event rate) is given $(1+A(\mathrm{NP}) / A(\mathrm{SM}))^{2}$. In terms of $\kappa_{\gamma}$ and $\kappa_{Z_{\gamma}}$ :

$$
\begin{gathered}
\kappa_{\gamma}=1.1525 \\
\kappa_{Z \gamma}=1.6107 .
\end{gathered}
$$

Applying the $4 \ell$ analysis, we obtain the results shown in Fig. 7.4. Specifically, we generated 1 million MC sample $p p \rightarrow h \rightarrow 4 l$ events for both the SM scenario and our singlet-triplet benchmark, and the liklihood was constructed using multiple pseudo-experiments with varying number of events $N$. In Fig. 7.4, the points in the top figure show the discrimination power between the SM and NP models presented in terms of Gaussian $\sigma$ as a function of $N$, the number of events. We also impose a fit curve of the form $x_{0}+x_{1} \sqrt{N}$ as $\sqrt{N}$ growth is expected for large $N$ with large signal to background, as is the case in $h \rightarrow 4 \ell$.

The curve from the top figure is extrapolated in the bottom part of Fig. 7.4 in 

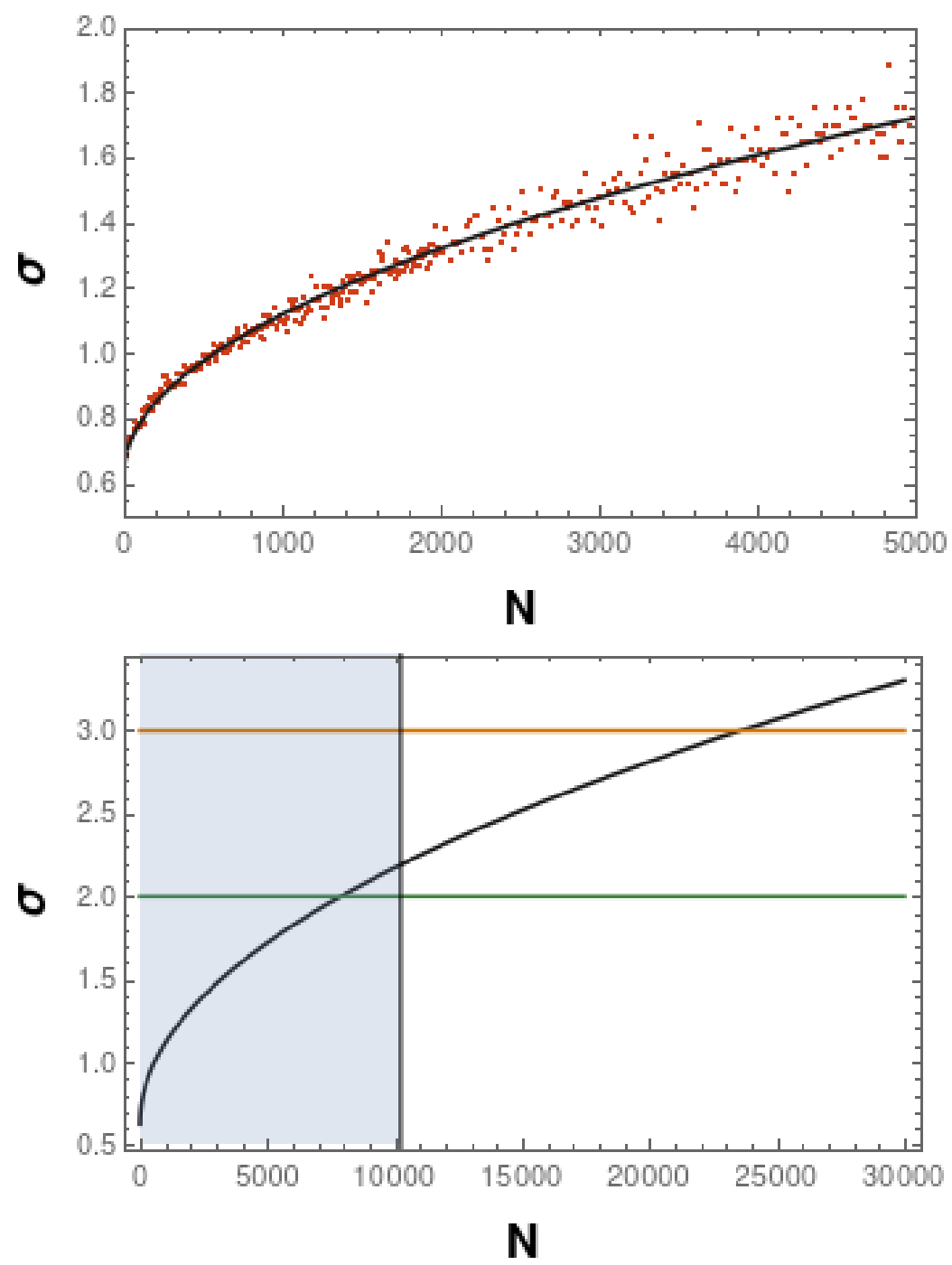

Figure 7.4: Number of Higgs to four lepton events $N$ required to distinguish the SM from a large $Z \gamma$ model benchmark at a given significance $\sigma$. The top figure shows the curve fitted to the average statistical significance of pseudo-experiments featuring a given number of events (the red points). The bottom figure shows an extrapolation of this data with lines at 2 and $3 \sigma$ significance. The blue shaded region shows the expected reach of the HL LHC.

order to estimate the required number of events to distinguish these two scenarios. With $\sim 8000$ events, we can rule out the parameter point at $2 \sigma$ and, more generally, we can begin to probe the large $Z \gamma$ model parameter space. Using $139 \mathrm{fb}^{-1}$ of data, ATLAS has measured the fiducial cross section of the $H \rightarrow Z Z^{*} \rightarrow 4 l$ to be $\sigma_{f i d}=$ 
$3.28 \pm 0.32 \mathrm{fb}$ in good agreement with the SM prediction $\sigma_{\text {fid }}=3.41 \pm 0.18 \mathrm{fb}[250]$. With the high luminosity (HL) LHC expected to collect upwards of $3000 \mathrm{fb}^{-1}$ over its lifetime [251], the LHC should be able to begin seriously probing the large $Z_{\gamma}$ parameter space by the end of its run.

At a future high energy hadron collider the prospects improve further. At $100 \mathrm{TeV}$ center of mass, the expected gluon fusion production cross section is $\sigma=$ $808.23_{-56.95}^{+44.53} \mathrm{pb}[252]$ with additional contributions of $\sim 10 \%$ total from vector boson fusion and associated production [253]. Taking the $h \rightarrow 4 l$ branching ratio to be

$2.796 \times 10^{-4}[254]$ and assuming an integrated luminosity of $10 \mathrm{ab}^{-1}$ gives $\sim 2.5$ million signal events - enough to potentially probe the relevant parameter space of this model. With this level of precision, other effects not considered here such as backgrounds, detector effects, and higher order corrections may become important.

\subsection{Supersymmetric Models}

We now turn to study supersymmetric models [10] to see if large effects in $h \rightarrow$ $Z \gamma$ can appear in well motivated models. Using the analysis techniques of Sec. 7.3, we examine scalar top partners which, in the MSSM, generically have the largest coupling to the Higgs of new supersymmetric states. Unsurprisingly, the additional restrictions coming from the extra structure present in SUSY models leads to much smaller allowed contributions to $h \rightarrow Z \gamma$ than with the general multiplet scenarios. In addition, for SUSY models we find that the deviations in the branching ratio of $h \rightarrow Z \gamma$ are always smaller than the deviations in $h \rightarrow \gamma \gamma$.

In addition to considering stops in the MSSM, we also consider F-stops, scalar top partners in folded SUSY models [228]. This class of models stabilizes the weak scale against radiative corrections to around $5 \mathrm{TeV}$. The scalar partners in these mod- 
els are not charged under $\mathrm{SU}(3)_{c}$, but have the same electroweak quantum numbers as stops allowing them to couple to the Higgs in the same way. This results in F-SUSY contributions to the Higgs decays to two photons and $Z \gamma$, but no modification to the $g g \rightarrow h$ amplitude (although there is a new decay to hidden gluons, $h \rightarrow g_{h} g_{h}$ ). There are many versions of F-SUSY to chose from; here we take the simplest approach and imagine a scenario identical to the MSSM stop sector sans stop-gluon couplings [227]. We examine the current restrictions on the stop parameter space for both models and then complete a four lepton analysis to estimate the required number of Higgs events to probe these scenarios.

The (F)-stop parameter space is dominantly controlled by three parameters: the two mass eigenvalues $m_{1}, m_{2}$, and the mixing angle between the gauge and mass eigenstates, $\theta_{t}$ (we use the conventions in [62]). There is some weak dependence on $\tan \beta$ - the couplings change less than $10 \%$ over any possible $\beta$ value and, if we restrict this parameter to its normally accepted values, $3 \leq \tan \beta \leq 50$, the modification becomes $\mathcal{O}(1 \%)$. As such, we take $\tan \beta=10$ for simplicity.

Using standard formulas in the literature we can compute the contributions of stops to $g g \rightarrow h$ and $h \rightarrow \gamma \gamma$ and apply the ATLAS constraints shown in Fig. 7.3. We ignore direct bounds on (F)-stops in order to keep our constraints model independent. As this is a three dimensional parameter space, we present our results in Fig. 7.5 in two dimensional slices of the two mass eigenstates with the mixing angle fixed at $\theta_{t}=0$ (top) and the lighter mass eigenstate $m_{1}$ vs. the mixing angle $\theta_{t}$ with $m_{2}=1$ $\mathrm{TeV}$ (bottom). This is an update of the analysis in [227] using significantly more data. Scanning the parameter space indicates that MSSM stops below $\sim 140 \mathrm{GeV}$ are essentially excluded. Additionally, both stops having a mass under $\sim 200 \mathrm{GeV}$ is excluded. In general, the weakest parameter space constraints occur when either mixing is maximized or the stops have a very large mass spliting and are effectively 
decoupled.

We can now compute the contribution to the $C P$-even form factor $A_{2}$ from Eq. (7.9). ${ }^{4}$ By scanning over the $m_{1}, m_{2}$, and $\theta_{t}$ parameter space and subjecting the results to the previously mentioned ATLAS constraints, the maximal modification of said form factor (and thus matrix element) was found to be:

$$
\begin{gathered}
\left|\frac{A_{2}^{\gamma \gamma}(\text { stop })}{A_{2}^{\gamma \gamma}(\mathrm{SM})}\right| \lesssim 9 \% \\
\left|\frac{A_{2}^{Z \gamma}(\text { stop })}{A_{2}^{Z \gamma}(\mathrm{SM})}\right| \lesssim 4 \% .
\end{gathered}
$$

This is beyond the precision expected on the coupling $\kappa_{Z \gamma}$ from the HL-LHC of $\mathcal{O}(10 \%)$ [230], but deviations from the SM in this channel may be visible at a 100 $\mathrm{TeV}$ collider with an expected precision of $\mathcal{O}(1 \%)$ [256]. If this is the theory of nature, we expect to see deviation from the SM in $h \rightarrow \gamma \gamma$ long before we see one in $h \rightarrow Z \gamma$.

For F-stops the procedure is the same but we can ignore constraints from gluon fusion production of the Higgs. This makes measurements less constricting leading to the results presented with the dashed lines in Fig. 7.5. The notably weaker constraints lead to stops only below $\sim 100 \mathrm{GeV}$ being universally excluded and requiring at least one stop above $\sim 150 \mathrm{GeV}$. Other than the strength of the bounds, the overall takeaways remain the same as with MSSM stops: the weakest constraints on the lighter stop occur when either mixing angle has values of $\theta_{t}=0, \pm \frac{\pi}{2}$ or the stops have a very large mass splitting. The $A_{2}$ values in the F-stop allows greater deviations:

$$
\begin{aligned}
& \left|\frac{A_{2}^{\gamma \gamma}(\mathrm{F} \text {-stop })}{A_{2}^{\gamma \gamma}(\mathrm{SM})}\right| \lesssim 14 \% \\
& \left|\frac{A_{2}^{Z \gamma}(\mathrm{F} \text {-stop })}{A_{2}^{Z \gamma}(\mathrm{SM})}\right| \lesssim 9 \% .
\end{aligned}
$$

\footnotetext{
${ }^{4} C P$ violation can exist in the MSSM but the constraints are strong [255].
} 
but we see that the deviations in $h \rightarrow Z \gamma$ are much smaller than the singlet triplet model shown in Eq. (7.30). This is just on the edge of the sensitivity of the HL-LHC in on-shell $h \rightarrow Z \gamma$ [230], but deviations would first be seen in $h \rightarrow \gamma \gamma$.

\subsubsection{Four-Lepton Analysis}

The four-lepton analysis of the SUSY models follows the one in Sec. 7.3. For stops, we use the following benchmark point:

$$
\begin{aligned}
& \text { - } m_{1}=177.8 \mathrm{GeV} \\
& \text { - } m_{2}=184.0 \mathrm{GeV} \\
& \text { - } \theta_{t}=-1.435 .
\end{aligned}
$$

which gives form factors

$$
\begin{aligned}
& \frac{A_{2}^{\gamma \gamma}(\text { stop })}{A_{2}^{\gamma \gamma}(\mathrm{SM})}=\frac{0.00072}{-0.008}=-0.09 \\
& \frac{A_{2}^{Z \gamma}(\text { stop })}{A_{2}^{Z \gamma}(\mathrm{SM})}=\frac{0.00028}{-0.014}=-0.02 \\
& \frac{A_{2}^{g g}(\mathrm{stop})}{A_{2}^{g g}(\mathrm{SM})}=\frac{0.00075}{0.0127}=0.0593
\end{aligned}
$$

where we have introduced the gluon form factor $A_{2}^{g g}$ analogous to those of the electroweak gauge bosons. Similarly, the point chosen for F-stops has parameters,

$$
\begin{aligned}
& \text { - } m_{1}=126.2 \mathrm{GeV} \\
& \text { - } m_{2}=180.6 \mathrm{GeV} \\
& \text { - } \theta_{t}=-1.285 .
\end{aligned}
$$


which gives:

$$
\begin{aligned}
& \frac{A_{2}^{\gamma \gamma}(\text { F-stop })}{A_{2}^{\gamma \gamma}(\mathrm{SM})}=\frac{0.00115}{-0.008}=-0.1437 \\
& \frac{A_{2}^{Z \gamma}(\mathrm{F} \text {-stop })}{A_{2}^{Z \gamma}(\mathrm{SM})}=\frac{0.00082}{-0.014}=-0.0586
\end{aligned}
$$

As in the large $Z \gamma$ case, these coupling modifications were used in generating $\mathrm{MC}$ events that were then processed into likelihoods, test statistic distributions, and, finally, statistical confidence as a function of number of signal events $\sigma(N)$. The results for both stop and F-stop scenarios are shown in Fig. 7.6. Unsurprisingly, the much smaller impact of the intermediate scalar particles within the loop decays makes both the stop and F-stop much more difficult to probe than the large $Z \gamma$ models. With the HL-LHC's integrated luminosity topping out around $3000 \mathrm{fb}^{-1}$ after its final run [251], the required $\sim 150$ thousand events to be sensitive F-stops or $\sim 270$ thousand events required to explore stops at $2 \sigma$ are not reachable with the LHC. These numbers are, however, potentially in reach of a future $100 \mathrm{TeV}$ collider

- having an integrated luminosity on the order $10 \mathrm{ab}^{-1}$ gives $\sim 2.5$ million signal events which can potentially probe the SUSY parameter space.

\subsection{Conclusion}

We have explored the possibility of new physics models with significant enhancements to the $h \rightarrow Z \gamma$ decay. We have demonstrated that simple models cannot give rise to large enhancements while still being consistent with other data, particularly measurements of $h \rightarrow \gamma \gamma$. Models with multiple multiplets featuring auspicious cancellations could produce such a signal. For models that do feature large contributions to $h \rightarrow Z \gamma$, such as a model with a singlet and a triplet explored in section 7.3.3, 
kinematic analysis of $h \rightarrow 4 \ell$ will be able to probe these models with the data from the high-luminosity LHC.

We also explored more motivated models that can solve the hierarchy problem, focusing in particular on stop contributions in supersymmetry and colourless top partners (F-stops) in folded SUSY. We used current Higgs data to constrain the parameter space of those states updating the analysis of [227], and determined these models cannot give measurable deviations to $h \rightarrow Z \gamma$. Kinematic analysis of $h \rightarrow$ $4 \ell$ can only have sensitivity to these models with significantly more data than the LHC will have although this may be possible with a future $100 \mathrm{TeV}$ hadron collider.

Although this work focused exclusively on the new physics contributions of scalars, an analagous analysis with fermionic multiplets would not change the qualitative conclusions: the loop factors and Higgs couplings are slightly different, but the photon and $Z$ couplings still arise from the covariant derivative and models with large contributions to $h \rightarrow Z \gamma$ can only be produced through fortuitous cancellation by multiple new physics multiplets. $C P$ violating couplings were also not examined in this work, but the constraints on Higgs couplings due to EDM measurements [235] indicate that such couplings would have to be tiny - much too small to achieve the large $Z \gamma$ contributions that we examined.

What this ultimately means is that, in general, the $h \rightarrow Z \gamma$ channel is unlikely to be a place where new physics is discovered: simple or motivated models tend to lead to contributions smaller than the much more sensitive diphoton channel. As such, a discovery of a significant NP contribution to this channel is a strong indication that interference effects are present in the NP sector and points towards non-minimal models like those presented in this paper. Finally, the discriminating power of a $100 \mathrm{TeV}$ collider, particularly in $h \rightarrow 4 \ell$, is undeniable: where the LHC cannot even probe the most favourable parameter points of the MSSM, a future collider will collect 
significantly more events, potentially giving us a much clearer handle on the nature of our microscopic world. 

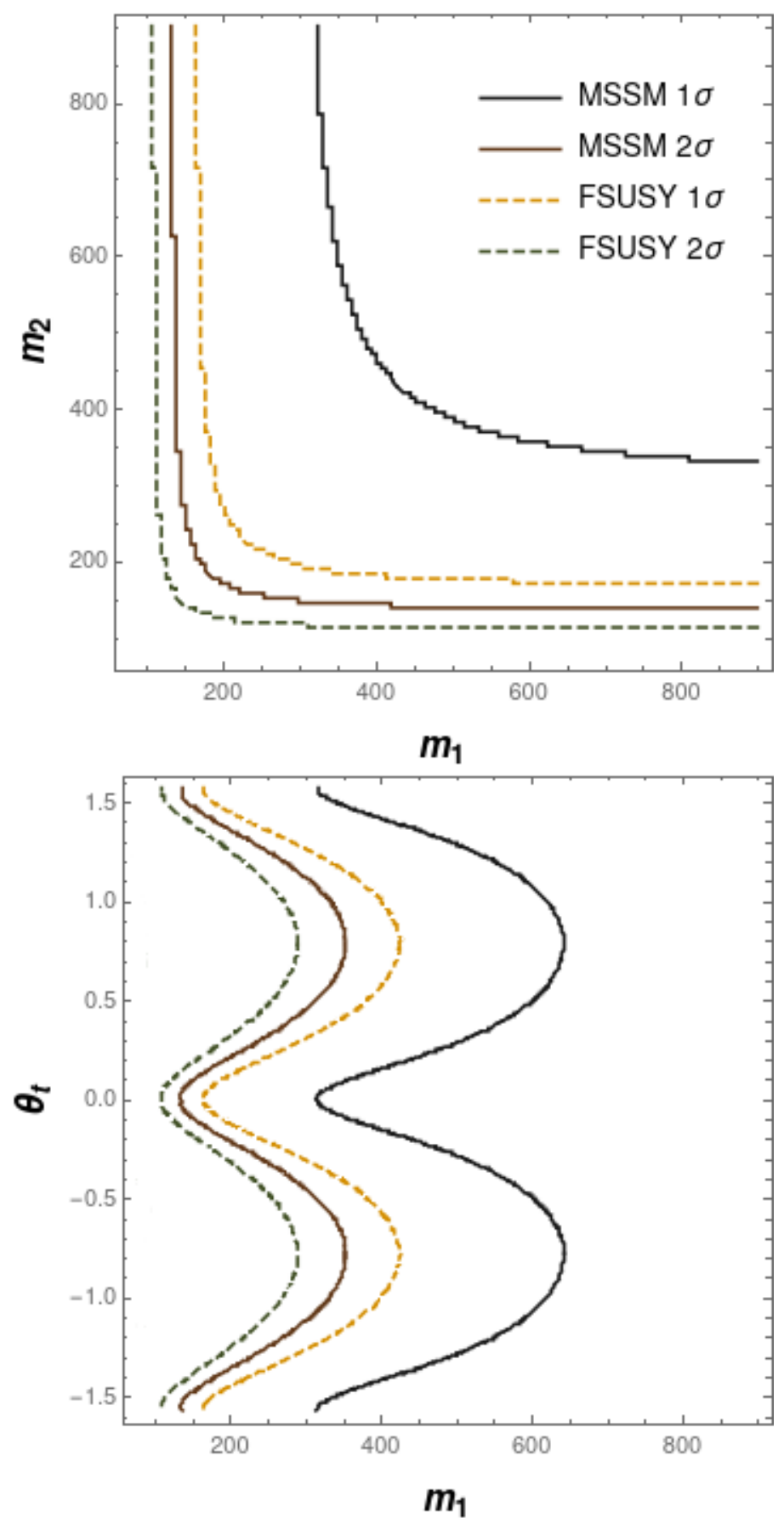

Figure 7.5: Exclusion bounds in stop parameter space due to $h \rightarrow \gamma \gamma$ and gluon fusion measurements: the solid rust (black) lines represent the 2 (1) sigma exclusion bounds for stops and the dashed green (yellow) lines represent the 2 (1) sigma exclusion bounds for F-stops. The top figure is in the stop mass 1, stop mass 2 plane with zero mixing. The bottom figure is in the stop mass 1 , stop mixing angle plane, with stop mass 2 held at $1 \mathrm{TeV}$. The region to the left of the curves is excluded. 

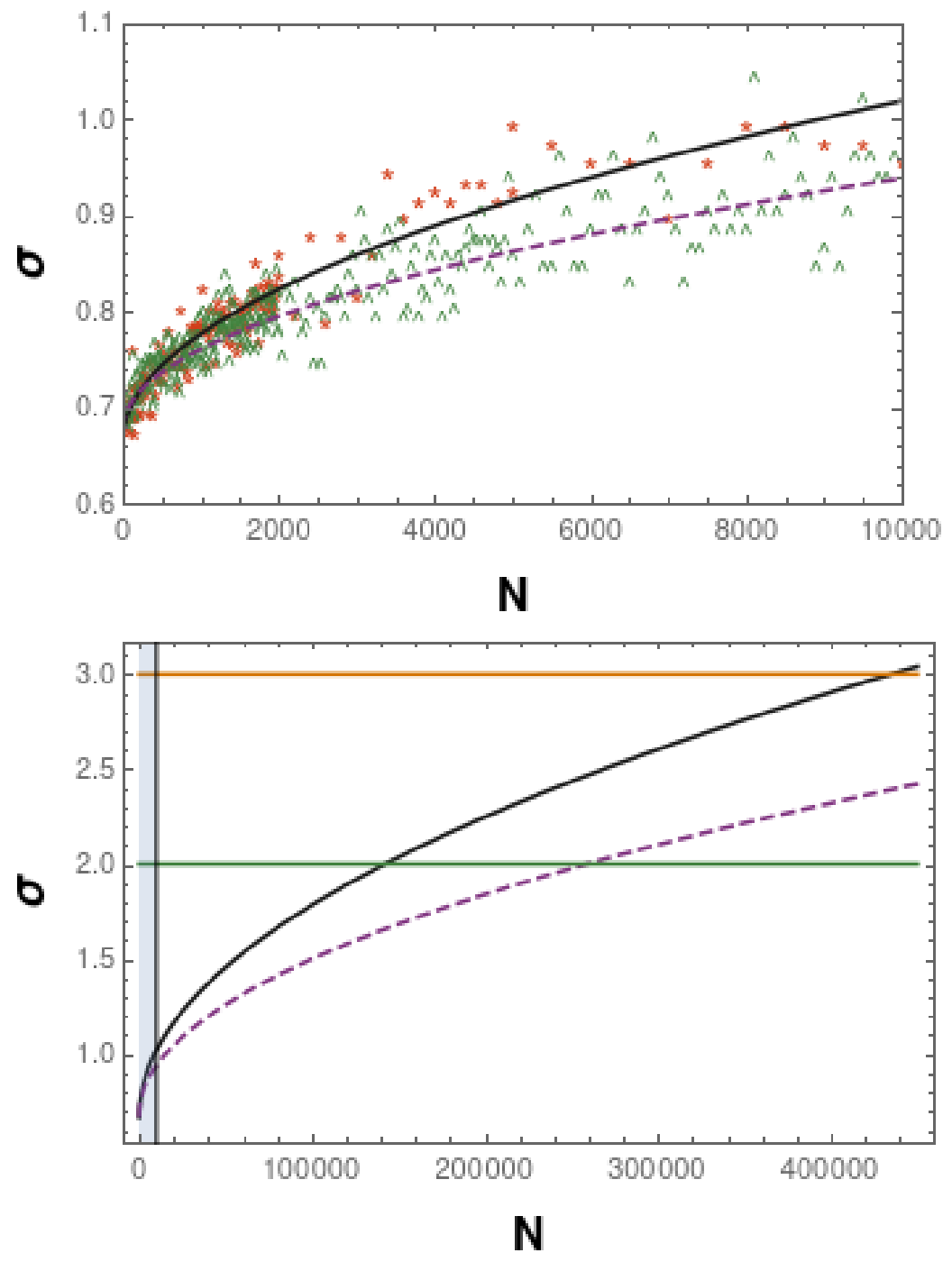

Figure 7.6: Number of Higgs to four lepton events $N$ required to distinguish the SM from the SUSY model benchmarks at a given significance $\sigma$. The top figure shows the curve fitted to the average statistical significance of pseudo-experiments featuring a given number of events. The dashed violet line (fit to the green carets) is the stop scenario and the solid black line (fit to the red asterisks) is the F-stop scenario. The bottom figure shows an extrapolation of this data with lines at 2 and $3 \sigma$ significance. The thin blue band on the left is the reach of the HL-LHC. 


\section{Chapter 8}

\section{Summary of Results}

Over the last several chapters, we have explored a kaleidoscope of BSM models in

all their vivid, colourful, and symmetric glory. The focus has been on the interesting signals that these models can produce, most notably gravitational waves, Cherenkov radiation in neutrino detectors, and higgs decays at the LHC.

In the study of GW from multiple hidden sectors, we demonstrated that although vanilla Nnaturalness did not produce GW that could be detectable in the near future, more generalized scenarios with multiple hidden sector SFOPTs are in a region accessible to next-generation space experiments. Both cases feature important parts of their GW signals in the void between frequencies detectable by pulsar timing arrays and space-based interferometers - providing theoretical impetus for new experiments capable of probing this region of frequency space. Additionally, this work demonstrated the power of GW signals to probe deep into the unknown arena of complex hidden sectors. Although individual SFOPTs are understood to create GWs that are expected to follow an approximate power law, if a model predicts the presence of two, five, or more SFOPTs (these can be in the same or different sectors) deviations from a standard power law can occur. The multiple transitions that occurred in the 
models outlined generated signals of this sort: although the individual GWs do obey approximate power laws, their sum does not - leading to a unique signal indicating so-called dark complexity. Explicitly, a broadening or distortion of the signal around the peak frequency, precisely where the signal has the most energy, could point to a multi-SFOPT scenario and gently guide us in the direction of multiple hidden sectors.

In the second paper, we proposed broadening the purpose of the Hyper Kamiokande experiment though using it to hunt down long-lived Higgs portal scalar particles produced from the atmosphere. The targeted parameter space was for the scalar mass below twice the muon mass that is allowed by existing searches. Scalars of this type could decay into an electron-positron pair in the bulk volume of the Hyper-K detector, creating a double-ring signal. We made approximations to the atmospheric production picture and computed a semi-analytical expression for the signal rate. Ultimately, we showed that for most events, the electron-positron opening angle is large enough for the double-ring signal to be resolved and that most of the decaying scalars would arrive from directions above the detector's horizon.

Finally, we explored the possibility of new physics models with significant enhancements to the $h \rightarrow Z \gamma$ decay. Crucially, we demonstrated that simple models cannot give rise to large enhancements while still being consistent with other data, particularly measurements of $h \rightarrow \gamma \gamma$. Models with multiple multiplets featuring auspicious cancellations can, however, produce such a signal. For models that do feature large contributions to $h \rightarrow Z \gamma$, kinematic analysis of $h \rightarrow 4 \ell$ will be able to probe these models with the data from the high-luminosity LHC. We also explored more motivated models that can solve the hierarchy problem, focusing in particular on stop contributions in supersymmetry and colourless top partners (F-stops) in folded SUSY. We used current higgs data to constrain the parameter space and determined these models cannot give measurable deviations to $h \rightarrow Z \gamma$. Kinematic analysis of 
$h \rightarrow 4 \ell$ can only have sensitivity to these models with significantly more data than the LHC will have although this may be possible with a future $100 \mathrm{TeV}$ hadron collider. Ultimately, this indicates that the $h \rightarrow Z_{\gamma}$ channel is unlikely to be a place where new physics is discovered: simple or motivated models tend to lead to contributions smaller than the much more sensitive diphoton channel. As such, a discovery of a significant NP contribution to this channel is a strong indication that interference effects are present in the NP sector and points towards non-minimal models. 


\section{Chapter 9}

\section{Epilogue}

With all the papers written, all the calculations completed, and all the plots constructed, the time has come to put the physics in the rear-view mirror, take a step back, and ask the perniciously difficult question of "why?". After all, we live on a rapidly heating globe - a pale blue dot nearly abandoned in an obsidian sky — torn to shreds by needless war, famine, and near limitless greed. Species are falling extinct at an incredibly rapid rate as an ecocide driven by the engines of industry gnaws away; the human and environmental costs of its function ignored as mere externalities. Even if we limit the scope to our little corner of the world that remains shielded from the direct consequences of this destruction, a simple glance is all it takes to note the structural violence of our own society and its cruelty towards the poor and the marginalized. So, in a world of pandemics and pain, what is the role of physics?

In this light, this is where physics becomes art. A task just as pointless as charcoal, just as frivolous as cinema, just as senseless as literature - yet, at the same time, every bit as beautiful. Now, here is where I must come into contention with the more common line about the beauty of physics (and other art too, I suppose): the beauty doesn't come from symmetry, nor the way the math comes together so 
smoothly, or even how the universe on the grandest of scales is at the whims of what takes place at the smallest.

No.

While all these pieces are aesthetic in their own right and have a certain elegance in their harmony, I don't buy that this is the true source of their beauty. Instead, I think it grows from the human side of the field: the countless hours poured in by generation after generation, the arguments, confusion, headaches, frustrations, wrong ideas, sleepless nights, broken hearts, and "wasted" lives. The willingness to work together to build ever larger experiments and fight tooth-and-nail for the construction of a new detector that has a minuscule chance of discovering something new.

All this just because we want to understand our place in the universe.

The beauty is the senselessness. 


\section{Appendix A}

\section{Conventions}

These are the conventions and notations used in this thesis.

First and foremost, we adopt the convention that $\hbar=c=1$ in order to simplify expressions. Secondly, the notion of distance in Minkowski space time is taken to have the following form:

$$
\mathrm{d} s^{2}=\mathrm{d} t^{2}-\left(\mathrm{d} x^{2}+\mathrm{d} y^{2}+\mathrm{d} z^{2}\right)
$$

Here, $x, y, z$ and $t$ are the coordinates of a Cartesian system $^{1}$. The above metric tensor leads us to our definition of 4 -vectors:

$$
x^{\mu}=\left(x^{0}, x^{1}, x^{2}, x^{3}\right)=(t, x, y, z) \text { and } x_{\mu}=\left(x_{0}, x_{1}, x_{2}, x_{3}\right)=(t,-x,-y,-z) .
$$

These vectors are related via the metric tensor of Minkowski space-time:

$$
x_{\mu}=g_{\mu \nu} x^{\nu} .
$$

\footnotetext{
${ }^{1}$ Chapter 3 uses the opposite, mostly plus convention.
} 
Thus, the metric tensor of the space is:

$$
g_{\mu \nu}=\left(\begin{array}{cccc}
1 & 0 & 0 & 0 \\
0 & -1 & 0 & 0 \\
0 & 0 & -1 & 0 \\
0 & 0 & 0 & -1
\end{array}\right) .
$$

Finally, we can define energy-momentum 4-vector

$$
p^{\mu}=(E, \mathbf{p})
$$




\section{Appendix B}

\section{Loop Integrals}

Here, for completeness, we present the integrated loop functions $l_{\gamma \gamma}$ and $l_{Z \gamma}$ for scalar NP particles of mass $m$ mentioned in Eq. (7.14). For further details, please see [257, 258].

The diphoton expression:

$$
l_{\gamma \gamma}[m]=\left(\frac{v}{4 \pi^{2} m_{h}^{4}}\right)\left(m_{h}^{2}-4 m^{2} \tan ^{-1}\left(\frac{m_{h}}{\sqrt{4 m^{2}-m_{h}^{2}}}\right)^{2}\right)
$$

And the $Z \gamma$ equation:

$$
\begin{aligned}
l_{Z \gamma}[m]= & \frac{v}{4 \pi^{2} m_{h}\left(m_{h}^{2}-m_{Z}^{2}\right)^{2}}\left\{m_{h}^{3}-2 m_{Z}^{2} \sqrt{4 m^{2}-m_{h}^{2}} \tan ^{-1}\left[\frac{m_{h}}{\sqrt{4 m^{2}-m_{h}^{2}}}\right]\right. \\
& -m_{h}\left[2 m^{2}\left[2 \tan ^{-1}\left[\frac{m_{h}}{\sqrt{4 m^{2}-m_{h}^{2}}}\right]^{2}-2 \tan ^{-1}\left[\frac{m_{Z}}{\sqrt{4 m^{2}-m_{Z}^{2}}}\right]^{2}\right]\right. \\
& \left.\left.-2 m_{Z} \sqrt{4 m^{2}-m_{Z}^{2}} \tan ^{-1}\left[\frac{m_{Z}}{\sqrt{4 m^{2}-m_{Z}^{2}}}\right]+m_{Z}^{2}\right]\right\}
\end{aligned}
$$

For the $Z \gamma$ expression, the case presented here describes loops with only one type of new particle; loops where the propagating particles within the loop are different 
particles lead to longer expressions we do not present here. In the case of the singlettriplet model presented in Sec. 7.3.3, this expression holds - however for the SUSY models in Sec. 7.4 the more general expression is required due to the possibility of the presence of both stops. 


\section{References}

[1] P. Archer-Smith, D. Linthorne, and D. Stolarski, Gravitational Wave Signals from Multiple Hidden Sectors, Phys. Rev. D 101 (2020), no. 9 095016, [arXiv: 1910.02083].

[2] P. Archer-Smith and Y. Zhang, Higgs Portal From The Atmosphere To Hyper-K, Phys. Lett. B 817 (2021) 136309, [arXiv:2005.08980].

[3] P. Archer-Smith, D. Stolarski, and R. Vega-Morales, On New Physics Contributions to the Higgs Decay to $Z \gamma$, Submitted to JHEP $(12,2020)$ [arXiv: 2012.01440].

[4] I. Newton, Philosophiae naturalis principia mathematica. J. Societatis Regiae ac Typis J. Streater, 1687.

[5] ATLAS, G. Aad et al., Observation of a new particle in the search for the Standard Model Higgs boson with the ATLAS detector at the LHC, Phys. Lett. B 716 (2012) 1-29, [arXiv:1207.7214].

[6] CMS, S. Chatrchyan et al., Observation of a New Boson at a Mass of 125 GeV with the CMS Experiment at the LHC, Phys. Lett. B 716 (2012) 30-61, [arXiv: 1207.7235].

[7] Planck, N. Aghanim et al., Planck 2018 results. VI. Cosmological parameters, arXiv: 1807.06209.

[8] Super-Kamiokande, Y. Fukuda et al., Evidence for oscillation of atmospheric neutrinos, Phys. Rev. Lett. 81 (1998) 1562-1567, [hep-ex/9807003].

[9] SNO, Q. R. Ahmad et al., Measurement of the rate of $\nu_{e}+d \rightarrow p+p+e^{-}$ interactions produced by ${ }^{8} B$ solar neutrinos at the Sudbury Neutrino Observatory, Phys. Rev. Lett. 87 (2001) 071301, [nucl-ex/0106015].

[10] S. P. Martin, A Supersymmetry primer, hep-ph/9709356. [Adv. Ser. Direct. High Energy Phys.18,1(1998)]. 
[11] P. W. Higgs, Spontaneous Symmetry Breakdown without Massless Bosons, Physical Review 145 (May, 1966) 1156-1163.

[12] S. L. Glashow, Partial-symmetries of weak interactions, Nuclear Physics 22 (1961), no. 4 579-588.

[13] S. Weinberg, A model of leptons, Phys. Rev. Lett. 19 (Nov, 1967) 1264-1266.

[14] M. E. Peskin and D. V. Schroeder, An introduction to quantum field theory. Westview Press Reading (Mass.), Boulder (Colo.), 1995.

[15] M. D. Schwartz, Quantum Field Theory and the Standard Model. Cambridge University Press, 2013.

[16] H. E. Logan, TASI 2013 lectures on Higgs physics within and beyond the Standard Model, arXiv:1406.1786.

[17] S. Isoyama, H. Nakano, and T. Nakamura, Multiband gravitational-wave astronomy: Observing binary inspirals with a decihertz detector, B-DECIGO, Progress of Theoretical and Experimental Physics 2018 (07, 2018).

[18] R. P. Feynman, Relativistic cut-off for quantum electrodynamics, Phys. Rev. 74 (Nov, 1948) 1430-1438.

[19] J. Schwinger, Quantum electrodynamics. i. a covariant formulation, Phys. Rev. 74 (Nov, 1948) 1439-1461.

[20] H. Goldstein, Classical Mechanics. Addison-Wesley, 1980.

[21] E. Wigner, On unitary representations of the inhomogeneous lorentz group, Annals of Mathematics 40 (1939), no. 1 149-204.

[22] Particle Data Group, P. Zyla et al., Review of Particle Physics, PTEP 2020 (2020), no. 8083 C01.

[23] M. Kobayashi and T. Maskawa, CP-Violation in the Renormalizable Theory of Weak Interaction, Progress of Theoretical Physics 49 (02, 1973) 652-657.

[24] N. Cabibbo, Unitary symmetry and leptonic decays, Phys. Rev. Lett. 10 (Jun, 1963) 531-533.

[25] Z. Maki, M. Nakagawa, and S. Sakata, Remarks on the Unified Model of Elementary Particles, Progress of Theoretical Physics 28 (11, 1962) 870-880.

[26] T. Cohen, As Scales Become Separated: Lectures on Effective Field Theory, PoS TASI2018 (2019) 011, [arXiv:1903.03622].

[27] E. W. Kolb and M. S. Turner, The Early Universe, vol. 69. 1990. 
[28] J. B. Hartle, Gravity: An Introduction to Einstein's General Relativity. Benjamin Cummings, 2003.

[29] M. Trodden and S. M. Carroll, TASI lectures: Introduction to cosmology, in Theoretical Advanced Study Institute in Elementary Particle Physics (TASI 2002): Particle Physics and Cosmology: The Quest for Physics Beyond the Standard Model(s), 1, 2004. astro-ph/0401547.

[30] J. M. Cline, TASI Lectures on Early Universe Cosmology: Inflation, Baryogenesis and Dark Matter, PoS TASI2018 (2019) 001, [arXiv:1807.08749].

[31] E. Hubble, A relation between distance and radial velocity among extra-galactic nebulae, Proceedings of the National Academy of Sciences 15 (1929), no. 3 168-173, [https://www.pnas.org/content/15/3/168.full.pdf].

[32] L. Husdal, On Effective Degrees of Freedom in the Early Universe, Galaxies 4 (2016), no. 4 78, [arXiv:1609.04979].

[33] P. F. de Salas and S. Pastor, Relic neutrino decoupling with flavour oscillations revisited, JCAP 07 (2016) 051, [arXiv:1606.06986].

[34] A. Einstein, Näherungsweise Integration der Feldgleichungen der Gravitation, Sitzungsberichte der Königlich Preußischen Akademie der Wissenschaften (Berlin (Jan., 1916) 688-696.

[35] A. Einstein, Über Gravitationswellen, Sitzungsber. Preuss. Akad. Wiss. Berlin (Math. Phys.) 1918 (1918) 154-167.

[36] Y. Aoki, G. Endrodi, Z. Fodor, S. D. Katz, and K. K. Szabo, The Order of the quantum chromodynamics transition predicted by the standard model of particle physics, Nature 443 (2006) 675-678, [hep-lat/0611014].

[37] T. Bhattacharya et al., QCD Phase Transition with Chiral Quarks and Physical Quark Masses, Phys. Rev. Lett. 113 (2014), no. 8082001 , [arXiv:1402.5175].

[38] K. Kajantie, M. Laine, K. Rummukainen, and M. E. Shaposhnikov, The Electroweak phase transition: A Nonperturbative analysis, Nucl. Phys. B 466 (1996) 189-258, [hep-lat/9510020].

[39] K. Kajantie, M. Laine, K. Rummukainen, and M. E. Shaposhnikov, Is there a hot electroweak phase transition at $m(H)$ larger or equal to $m(W)$ ?, Phys. Rev. Lett. 77 (1996) 2887-2890, [hep-ph/9605288].

[40] LIGO Scientific, J. Aasi et al., Advanced LIGO, Class. Quant. Grav. 32 (2015) 074001, [arXiv:1411.4547]. 
[41] eLISA, P. A. Seoane et al., The Gravitational Universe, arXiv:1305.5720.

[42] LIGO-Caltech, "Ligo-caltech info page." https://www.ligo.caltech.edu/page/what-is-interferometer. Accessed: 2021-06-13.

[43] B. Allen and J. D. Romano, Detecting a stochastic background of gravitational radiation: Signal processing strategies and sensitivities, Phys. Rev. D 59 (Mar, 1999) 102001.

[44] LISA, H. Audley et al., Laser Interferometer Space Antenna, arXiv: 1702.00786.

[45] J. Crowder and N. J. Cornish, Beyond lisa: Exploring future gravitational wave missions, Phys. Rev. D 72 (Oct, 2005) 083005.

[46] S. Sato et al., The status of DECIGO, Journal of Physics: Conference Series 840 (may, 2017) 012010.

[47] E. Thrane and J. D. Romano, Sensitivity curves for searches for gravitational-wave backgrounds, Phys. Rev. D 88 (Dec, 2013) 124032.

[48] T. Robson, N. J. Cornish, and C. Liu, The construction and use of LISA sensitivity curves, Classical and Quantum Gravity 36 (apr, 2019) 105011.

[49] K. Yagi, N. Tanahashi, and T. Tanaka, Probing the size of extra dimensions with gravitational wave astronomy, Phys. Rev. D 83 (Apr, 2011) 084036.

[50] K. Yagi, Scientific potential of decigo pathfinder and testing gr with space-borne gravitational wave interferometers, International Journal of Modern Physics D 22 (2013), no. 011341013.

[51] M. Breitbach, J. Kopp, E. Madge, T. Opferkuch, and P. Schwaller, Dark, Cold, and Noisy: Constraining Secluded Hidden Sectors with Gravitational Waves, arXiv: 1811.11175.

[52] G. Janssen et al., Gravitational wave astronomy with the SKA, PoS AASKA14 (2015) 037, [arXiv:1501.00127].

[53] B. Patt and F. Wilczek, Higgs-field portal into hidden sectors, hep-ph/0605188.

[54] J. Beacham et al., Physics Beyond Colliders at CERN: Beyond the Standard Model Working Group Report, J. Phys. G 47 (2020), no. 1 010501, [arXiv: 1901.09966]. 
[55] S. Weinberg, Goldstone Bosons as Fractional Cosmic Neutrinos, Phys. Rev. Lett. 110 (2013), no. 24 241301, [arXiv:1305.1971].

[56] G. Arcadi, A. Djouadi, and M. Raidal, Dark Matter through the Higgs portal, Phys. Rept. 842 (2020) 1-180, [arXiv:1903.03616].

[57] N. Arkani-Hamed, T. Cohen, R. T. D'Agnolo, A. Hook, H. D. Kim, and D. Pinner, Solving the Hierarchy Problem at Reheating with a Large Number of Degrees of Freedom, Phys. Rev. Lett. 117 (2016), no. 25 251801, [arXiv: 1607.06821].

[58] K. C. Freeman, On the disks of spiral and s0 galaxies, apj 160 (June, 1970) 811.

[59] V. C. Rubin and J. Ford, W. Kent, Rotation of the andromeda nebula from a spectroscopic survey of emission regions, apj 159 (Feb., 1970) 379.

[60] K. Earl, Exploring supersymmetry and naturalness in light of new experimental data. PhD thesis, 2019.

[61] M. Kuroda, Complete Lagrangian of MSSM, - (1999) [hep-ph/9902340].

[62] B. Batell, M. McCullough, D. Stolarski, and C. B. Verhaaren, Putting a Stop to di-Higgs Modifications, JHEP 09 (2015) 216, [arXiv:1508.01208].

[63] LIGO Scientific, Virgo, B. P. Abbott et al., Observation of Gravitational Waves from a Binary Black Hole Merger, Phys. Rev. Lett. 116 (2016), no. 6 061102, [arXiv: 1602.03837].

[64] K. Danzmann, LISA: Laser interferometer space antenna for gravitational wave measurements, in Relativistic astrophysics and cosmology. Proceedings, 17th Symposium, Munich, Germany, December 11-17, 1997, pp. 481-484, 1994.

[65] N. Seto, S. Kawamura, and T. Nakamura, Possibility of direct measurement of the acceleration of the universe using 0.1-Hz band laser interferometer gravitational wave antenna in space, Phys. Rev. Lett. 87 (2001) 221103, [astro-ph/0108011].

[66] J. Crowder and N. J. Cornish, Beyond LISA: Exploring future gravitational wave missions, Phys. Rev. D72 (2005) 083005, [gr-qc/0506015].

[67] G. M. Harry, P. Fritschel, D. A. Shaddock, W. Folkner, and E. S. Phinney, Laser interferometry for the big bang observer, Class. Quant. Grav. 23 (2006) 4887-4894. [Erratum: Class. Quant. Grav.23,7361(2006)]. 
[68] P. Amaro-Seoane et al., Laser Interferometer Space Antenna, arXiv e-prints (Feb, 2017) arXiv:1702.00786, [arXiv:1702.00786].

[69] E. Witten, Cosmic Separation of Phases, Phys. Rev. D30 (1984) 272-285.

[70] C. J. Hogan, NUCLEATION OF COSMOLOGICAL PHASE TRANSITIONS, Phys. Lett. 133B (1983) 172-176.

[71] C. J. Hogan, Gravitational radiation from cosmological phase transitions, Mon. Not. Roy. Astron. Soc. 218 (1986) 629-636.

[72] M. S. Turner and F. Wilczek, Relic gravitational waves and extended inflation, Phys. Rev. Lett. 65 (Dec, 1990) 3080-3083.

[73] C. Caprini et al., Science with the space-based interferometer eLISA. II: Gravitational waves from cosmological phase transitions, JCAP 1604 (2016), no. 04 001, [arXiv: 1512.06239].

[74] A. Mazumdar and G. White, Review of cosmic phase transitions: their significance and experimental signatures, Rept. Prog. Phys. 82 (2019), no. 7 076901, [arXiv: 1811.01948].

[75] P. Schwaller, Gravitational Waves from a Dark Phase Transition, Phys. Rev. Lett. 115 (2015), no. 18 181101, [arXiv:1504.07263].

[76] J. Jaeckel, V. V. Khoze, and M. Spannowsky, Hearing the signal of dark sectors with gravitational wave detectors, Phys. Rev. D94 (2016), no. 10 103519, [arXiv: 1602.03901].

[77] A. Addazi, Limiting First Order Phase Transitions in Dark Gauge Sectors from Gravitational Waves experiments, Mod. Phys. Lett. A32 (2017), no. 08 1750049, [arXiv: 1607.08057].

[78] E. Hardy, Miniclusters in the Axiverse, JHEP 02 (2017) 046, [arXiv: 1609.00208].

[79] K. R. Dienes, F. Huang, S. Su, and B. Thomas, Dynamical Dark Matter from Strongly-Coupled Dark Sectors, Phys. Rev. D95 (2017), no. 4043526 , [arXiv:1610.04112].

[80] K. Tsumura, M. Yamada, and Y. Yamaguchi, Gravitational wave from dark sector with dark pion, JCAP 1707 (2017), no. 07 044, [arXiv: 1704.00219].

[81] B. S. Acharya, M. Fairbairn, and E. Hardy, Glueball dark matter in non-standard cosmologies, JHEP 07 (2017) 100, [arXiv:1704.01804]. 
[82] N. Bernal, M. Heikinheimo, T. Tenkanen, K. Tuominen, and V. Vaskonen, The Dawn of FIMP Dark Matter: A Review of Models and Constraints, Int. J. Mod. Phys. A32 (2017), no. 27 1730023, [arXiv:1706.07442].

[83] M. Aoki, H. Goto, and J. Kubo, Gravitational Waves from Hidden QCD Phase Transition, Phys. Rev. D96 (2017), no. 7 075045, [arXiv:1709.07572].

[84] M. Heikinheimo, K. Tuominen, and K. Langæble, Hidden strongly interacting massive particles, Phys. Rev. D97 (2018), no. 9 095040, [arXiv:1803.07518].

[85] M. Geller, A. Hook, R. Sundrum, and Y. Tsai, Primordial Anisotropies in the Gravitational Wave Background from Cosmological Phase Transitions, Phys. Rev. Lett. 121 (2018), no. 20 201303, [arXiv:1803.10780].

[86] D. Croon, V. Sanz, and G. White, Model Discrimination in Gravitational Wave spectra from Dark Phase Transitions, JHEP 08 (2018) 203, [arXiv: 1806.02332].

[87] I. Baldes and C. Garcia-Cely, Strong gravitational radiation from a simple dark matter model, JHEP 05 (2019) 190, [arXiv: 1809.01198].

[88] Y. Bai, A. J. Long, and S. Lu, Dark Quark Nuggets, Phys. Rev. D99 (2019), no. 5 055047, [arXiv:1810.04360].

[89] M. Fairbairn, E. Hardy, and A. Wickens, Hearing without seeing: gravitational waves from hot and cold hidden sectors, JHEP 07 (2019) 044, [arXiv: 1901.11038].

[90] A. J. Helmboldt, J. Kubo, and S. van der Woude, Observational prospects for gravitational waves from hidden or dark chiral phase transitions, arXiv: 1904.07891.

[91] A. Caputo and M. Reig, Cosmic implications of a low-scale solution to the axion domain wall problem, arXiv:1905.13116.

[92] G. Bertone et al., Gravitational wave probes of dark matter: challenges and opportunities, arXiv:1907.10610.


(2009) 055001, [arXiv:0905.1709].

[94] N. Craig, S. Knapen, and P. Longhi, Neutral Naturalness from Orbifold Higgs Models, Phys. Rev. Lett. 114 (2015), no. 6 061803, [arXiv:1410.6808].

[95] N. Craig, S. Knapen, and P. Longhi, The Orbifold Higgs, JHEP 03 (2015) 106, [arXiv: 1411.7393]. 
[96] D. Chialva, P. S. B. Dev, and A. Mazumdar, Multiple dark matter scenarios from ubiquitous stringy throats, Physical Review D 87 (Mar, 2013).

[97] K. R. Dienes and B. Thomas, Dynamical Dark Matter: I. Theoretical Overview, Phys. Rev. D85 (2012) 083523, [arXiv:1106.4546].

[98] K. R. Dienes and B. Thomas, Dynamical Dark Matter: II. An Explicit Model, Phys. Rev. D85 (2012) 083524, [arXiv:1107.0721].

[99] L. Susskind, Dynamics of Spontaneous Symmetry Breaking in the Weinberg-Salam Theory, Phys. Rev. D20 (1979) 2619-2625.

[100] D. J. Marsh, Axion cosmology, Physics Reports 643 (2016) 1 - 79. Axion cosmology.

[101] M. Geller, A. Hook, R. Sundrum, and Y. Tsai, Primordial anisotropies in the gravitational wave background from cosmological phase transitions, Phys. Rev. Lett. 121 (Nov, 2018) 201303.

[102] B. Svetitsky and L. G. Yaffe, Critical behavior at finite-temperature confinement transitions, Nuclear Physics B 210 (1982), no. 4423 - 447.

[103] R. D. Pisarski and F. Wilczek, Remarks on the Chiral Phase Transition in Chromodynamics, Phys. Rev. D29 (1984) 338-341.

[104] M. Panero, Thermodynamics of the QCD plasma and the large-N limit, Phys. Rev. Lett. 103 (2009) 232001, [arXiv:0907.3719].

[105] J.-W. Cui, H.-J. He, L.-C. Lu, and F.-R. Yin, Spontaneous Mirror Parity Violation, Common Origin of Matter and Dark Matter, and the LHC Signatures, Phys. Rev. D85 (2012) 096003, [arXiv:1110.6893].

[106] Particle Data Group, M. Tanabashi et al., Review of particle physics, Phys. Rev. D 98 (Aug, 2018) 030001.

[107] M. Gell-Mann, R. J. Oakes, and B. Renner, Behavior of current divergences under $\mathrm{su}_{3} \times \mathrm{su}_{3}$, Phys. Rev. 175 (Nov, 1968) 2195-2199.

[108] M. D. Schwartz, Quantum Field Theory and the Standard Model. Cambridge University Press, 2014.

[109] G. Mangano, G. Miele, S. Pastor, T. Pinto, O. Pisanti, and P. D. Serpico, Relic neutrino decoupling including flavor oscillations, Nucl. Phys. B729 (2005) 221-234, [hep-ph/0506164].

[110] CMB-S4, K. N. Abazajian et al., CMB-S4 Science Book, First Edition, arXiv: 1610.02743. 
[111] M. Trodden and S. M. Carroll, TASI lectures: Introduction to cosmology, in Progress in string theory. Proceedings, Summer School, TASI 2003, Boulder, USA, June 2-27, 2003, pp. 703-793, 2004. astro-ph/0401547. [,703(2004)].

[112] Z. Fodor and S. D. Katz, Lattice determination of the critical point of QCD at finite T and mu, JHEP 03 (2002) 014, [hep-lat/0106002].

[113] A. D. Linde, Decay of the False Vacuum at Finite Temperature, Nucl. Phys. B216 (1983) 421. [Erratum: Nucl. Phys.B223,544(1983)].

[114] J. R. Espinosa, T. Konstandin, J. M. No, and G. Servant, Energy Budget of Cosmological First-order Phase Transitions, JCAP 1006 (2010) 028, [arXiv: 1004.4187].

[115] C. Grojean and G. Servant, Gravitational waves from phase transitions at the electroweak scale and beyond, Phys. Rev. D 75 (Feb, 2007) 043507.

[116] A. Kosowsky, M. S. Turner, and R. Watkins, Gravitational radiation from colliding vacuum bubbles, Phys. Rev. D 45 (Jun, 1992) 4514-4535.

[117] S. J. Huber and T. Konstandin, Gravitational wave production by collisions: more bubbles, Journal of Cosmology and Astroparticle Physics 2008 (sep, 2008) 022 .

[118] M. Hindmarsh, S. J. Huber, K. Rummukainen, and D. J. Weir, Gravitational waves from the sound of a first order phase transition, Phys. Rev. Lett. 112 (Jan, 2014) 041301.

[119] C. Caprini and R. Durrer, Gravitational waves from stochastic relativistic sources: Primordial turbulence and magnetic fields, Phys. Rev. D 74 (Sep, 2006) 063521.

[120] D. Bödeker and G. D. Moore, Electroweak bubble wall speed limit, Journal of Cosmology and Astroparticle Physics 2017 (May, 2017) 025-025.

[121] M. Kamionkowski, A. Kosowsky, and M. S. Turner, Gravitational radiation from first-order phase transitions, Phys. Rev. D 49 (Mar, 1994) 2837-2851.

[122] M. Hindmarsh, S. J. Huber, K. Rummukainen, and D. J. Weir, Shape of the acoustic gravitational wave power spectrum from a first order phase transition, Phys. Rev. D96 (2017), no. 10 103520, [arXiv:1704.05871].

[123] J. Ellis, M. Lewicki, and J. M. No, On the Maximal Strength of a First-Order Electroweak Phase Transition and its Gravitational Wave Signal, arXiv: 1809.08242. [JCAP1904,003(2019)]. 
[124] J. Ellis, M. Lewicki, J. M. No, and V. Vaskonen, Gravitational wave energy budget in strongly supercooled phase transitions, Journal of Cosmology and Astroparticle Physics 2019 (jun, 2019) 024-024.

[125] T. Alanne, T. Hugle, M. Platscher, and K. Schmitz, A fresh look at the gravitational-wave signal from cosmological phase transitions, 2019.

[126] M. B. Wise and Y. Zhang, Stable Bound States of Asymmetric Dark Matter, Phys. Rev. D 90 (2014), no. 5 055030, [arXiv:1407.4121]. [Erratum: Phys.Rev.D 91, 039907 (2015)].

[127] M. B. Wise and Y. Zhang, Yukawa Bound States of a Large Number of Fermions, JHEP 02 (2015) 023, [arXiv: 1411.1772]. [Erratum: JHEP 10, 165 (2015)].

[128] Y. Zhang, Long-lived Light Mediator to Dark Matter and Primordial Small Scale Spectrum, JCAP 05 (2015) 008, [arXiv: 1502.06983].

[129] G. W. Anderson and L. J. Hall, The Electroweak phase transition and baryogenesis, Phys. Rev. D 45 (1992) 2685-2698.

[130] M. Pietroni, The Electroweak phase transition in a nonminimal supersymmetric model, Nucl. Phys. B 402 (1993) 27-45, [hep-ph/9207227].

[131] M. Carena, M. Quirós, and Y. Zhang, Electroweak Baryogenesis from Dark-Sector CP Violation, Phys. Rev. Lett. 122 (2019), no. 20 201802, [arXiv:1811.09719].

[132] M. Carena, M. Quirós, and Y. Zhang, Dark CP violation and gauged lepton or baryon number for electroweak baryogenesis, Phys. Rev. D 101 (2020), no. 5 055014, [arXiv: 1908.04818].

[133] D. Tucker-Smith and I. Yavin, Muonic hydrogen and MeV forces, Phys. Rev. D 83 (2011) 101702, [arXiv:1011.4922].

[134] C.-Y. Chen, H. Davoudiasl, W. J. Marciano, and C. Zhang, Implications of a light "dark Higgs" solution to the $g_{\mu}$-2 discrepancy, Phys. Rev. D 93 (2016), no. 3 035006, [arXiv:1511.04715].

[135] P. W. Graham, D. E. Kaplan, and S. Rajendran, Cosmological Relaxation of the Electroweak Scale, Phys. Rev. Lett. 115 (2015), no. 22 221801, [arXiv:1504.07551].

[136] T. Flacke, C. Frugiuele, E. Fuchs, R. S. Gupta, and G. Perez, Phenomenology of relaxion-Higgs mixing, JHEP 06 (2017) 050, [arXiv:1610.02025]. 
[137] J. D. Clarke, R. Foot, and R. R. Volkas, Phenomenology of a very light scalar $\left(100 \mathrm{MeV}<m_{h}<10 \mathrm{GeV}\right)$ mixing with the SM Higgs, JHEP 02 (2014) 123, [arXiv: 1310.8042].

[138] S. Shinohara, Search for the rare decay $k \rightarrow \pi^{0} \nu \bar{\nu}$ at $j$-PARC KOTO experiment, Journal of Physics: Conference Series 1526 (2020) 012002.

[139] KOTO, Shimizu, N., Search for new physics via the $k_{l} \rightarrow \pi_{0} \nu \bar{\nu}$ decay at the j-parc koto experiment, 2020.

[140] KOTO, S. Shinohara, Search for the rare decay $k_{l} \rightarrow \pi^{0} \nu \bar{\nu}$ at $j$-parc koto experiment, .

[141] KOTO, J. K. Ahn et al., Study of the $K_{L} \rightarrow \pi^{0} \nu \bar{\nu}$ decay at the J-PARC KOTO experiment, arXiv:2012.07571.

[142] D. Egana-Ugrinovic, S. Homiller, and P. Meade, Light Scalars and the KOTO Anomaly, Phys. Rev. Lett. 124 (2020), no. 19 191801, [arXiv:1911.10203].

[143] T. Kitahara, T. Okui, G. Perez, Y. Soreq, and K. Tobioka, New physics implications of recent search for $K_{L} \rightarrow \pi^{0} \nu \bar{\nu}$ at KOTO, Phys. Rev. Lett. 124 (2020), no. 7 071801, [arXiv: 1909.11111].

[144] P. B. Dev, R. N. Mohapatra, and Y. Zhang, Constraints on long-lived light scalars with flavor-changing couplings and the KOTO anomaly, Phys. Rev. D 101 (2020), no. 7 075014, [arXiv:1911.12334].

[145] J. Liu, N. McGinnis, C. E. Wagner, and X.-P. Wang, A Light Scalar Explanation of $(g-2)_{\mu}$ and the KOTO Anomaly, arXiv:2001.06522.

[146] BNL-E949, A. Artamonov et al., Study of the decay $K^{+} \rightarrow \pi^{+} \nu \bar{\nu}$ in the momentum region $140<P_{\pi}<199 \mathrm{MeV} / \mathrm{c}$, Phys. Rev. D 79 (2009) 092004, [arXiv: 0903.0030].

[147] NA62, E. Cortina Gil et al., An investigation of the very rare $K^{+} \rightarrow \pi^{+} \nu \bar{\nu}$ decay, JHEP 11 (2020) 042, [arXiv:2007.08218].

[148] NA62, E. Cortina Gil et al., Search for $\pi^{0}$ decays to invisible particles, arXiv:2010.07644.

[149] NA62, E. Cortina Gil et al., Search for a feebly interacting particle $X$ in the decay $K^{+} \rightarrow \pi^{+} X$, arXiv:2011.11329.

[150] CHARM, F. Bergsma et al., Search for Axion Like Particle Production in 400-GeV Proton - Copper Interactions, Phys. Lett. B 157 (1985) 458-462. 
[151] Hyper-Kamiokande, K. Abe et al., Hyper-kamiokande design report, arXiv: 1805.04163.

[152] T. Sjöstrand, S. Ask, J. R. Christiansen, R. Corke, N. Desai, P. Ilten, S. Mrenna, S. Prestel, C. O. Rasmussen, and P. Z. Skands, An Introduction to PYTHIA 8.2, Comput. Phys. Commun. 191 (2015) 159-177, [arXiv:1410.3012].

[153] Particle Data Group, M. Tanabashi et al., Review of Particle Physics, Phys. Rev. D 98 (2018), no. 3030001.

[154] H. Leutwyler and M. Shifman, Light Higgs particle in decays of $K$ and Eta mesons, Nuclear Physics B 343 (1990), no. 2369 - 397.

[155] J. L. Feng, I. Galon, F. Kling, and S. Trojanowski, Dark Higgs bosons at the ForwArd Search ExpeRiment, Phys. Rev. D 97 (2018), no. 5055034 , [arXiv: 1710.09387].

[156] B. Batell, J. Berger, and A. Ismail, Probing the Higgs Portal at the Fermilab Short-Baseline Neutrino Experiments, Phys. Rev. D 100 (2019), no. 11 115039, [arXiv:1909.11670].

[157] J. F. Gunion, H. E. Haber, G. L. Kane, and S. Dawson, The Higgs Hunter's Guide, vol. 80. 2000.

[158] Y. Grossman and Y. Nir, $K(L) \rightarrow \pi_{0}$ neutrino anti-neutrino beyond the standard model, Phys. Lett. B 398 (1997) 163-168, [hep-ph/9701313].

[159] C. Argüelles, P. Coloma, P. Hernández, and V. Muñoz, Searches for Atmospheric Long-Lived Particles, JHEP 02 (2020) 190, [arXiv:1910.12839].

[160] K. Abe et al., Atmospheric neutrino oscillation analysis with external constraints in super-kamiokande i-iv, Physical Review D 97 (Apr, 2018).

[161] S. Foroughi-Abari and A. Ritz, LSND Constraints on the Higgs Portal, Phys. Rev. D 102 (2020), no. 3 035015, [arXiv:2004.14515].

[162] LHCb, R. Aaij et al., Search for long-lived scalar particles in $B^{+} \rightarrow K^{+} \chi\left(\mu^{+} \mu^{-}\right)$decays, Phys. Rev. D 95 (2017), no. 7071101 , [arXiv: 1612.07818].

[163] LHCb, R. Aaij et al., Search for hidden-sector bosons in $B^{0} \rightarrow K^{* 0} \mu^{+} \mu^{-}$ decays, Phys. Rev. Lett. 115 (2015), no. 16 161802, [arXiv:1508.04094].

[164] Belle, K. F. Chen et al., Search for $B \rightarrow h^{(*)} \nu \bar{\nu}$ decays at Belle, Phys. Rev. Lett. 99 (2007) 221802, [arXiv:0707.0138]. 
[165] F. Bezrukov and D. Gorbunov, Light inflaton Hunter's Guide, JHEP 05 (2010) 010, [arXiv:0912.0390].

[166] M. A. Shifman, A. I. Vainshtein, and V. I. Zakharov, Remarks on Higgs Boson Interactions with Nucleons, Phys. Lett. B 78 (1978) 443-446.

[167] M. Dine, W. Fischler, and M. Srednicki, A Simple Solution to the Strong CP Problem with a Harmless Axion, Phys. Lett. B 104 (1981) 199-202.

[168] A. Zhitnitsky, THE WEINBERG MODEL OF THE CP VIOLATION AND T ODD CORRELATIONS IN WEAK DECAYS. (IN RUSSIAN), Sov. J. Nucl. Phys. 31 (1980) 529-534.

[169] Super-Kamiokande, Y. Fukuda et al., The Super-Kamiokande detector, Nucl. Instrum. Meth. A 501 (2003) 418-462.

[170] J. M. Berryman, A. de Gouvea, P. J. Fox, B. J. Kayser, K. J. Kelly, and J. L. Raaf, Searches for Decays of New Particles in the DUNE Multi-Purpose Near Detector, JHEP 02 (2020) 174, [arXiv: 1912.07622].

[171] MicroBooNE, P. Abratenko et al., Search for a Higgs portal scalar decaying to electron-positron pairs in the MicroBooNE detector, arXiv:2106.00568.

[172] ATLAS, M. Aaboud et al., Measurements of gluon-gluon fusion and vector-boson fusion Higgs boson production cross-sections in the $H \rightarrow W W^{*} \rightarrow e \nu \mu \nu$ decay channel in pp collisions at $\sqrt{s}=13$ TeV with the ATLAS detector, Phys. Lett. B 789 (2019) 508-529, [arXiv:1808.09054].

[173] ATLAS, G. Aad et al., Measurement of the production cross section for a Higgs boson in association with a vector boson in the $H \rightarrow W W^{*} \rightarrow \ell \nu \ell \nu$ channel in pp collisions at $\sqrt{s}=13$ TeV with the ATLAS detector, Phys. Lett. B 798 (2019) 134949, [arXiv: 1903.10052].

[174] CMS, A. M. Sirunyan et al., Measurements of properties of the Higgs boson decaying to a $W$ boson pair in pp collisions at $\sqrt{s}=13$ TeV, Phys. Lett. B 791 (2019) 96, [arXiv:1806.05246].

[175] CMS, A. M. Sirunyan et al., Measurement of the inclusive and differential Higgs boson production cross sections in the leptonic $W W$ decay mode at $\sqrt{s}=$ 13 TeV, arXiv:2007.01984.

[176] CMS, A. M. Sirunyan et al., Measurement and interpretation of differential cross sections for Higgs boson production at $\sqrt{s}=13$ TeV, Phys. Lett. B 792 (2019) 369-396, [arXiv: 1812.06504]. 
[177] ATLAS, G. Aad et al., Measurements of Higgs boson production and couplings in diboson final states with the ATLAS detector at the LHC, Phys. Lett. B 726 (2013) 88-119, [arXiv:1307.1427]. [Erratum: Phys.Lett.B 734, 406-406 (2014)].

[178] ATLAS, M. Aaboud et al., Measurements of Higgs boson properties in the diphoton decay channel with $36 \mathrm{fb}^{-1}$ of $p p$ collision data at $\sqrt{\mathrm{s}}=13 \mathrm{TeV}$ with the ATLAS detector, Phys. Rev. D98 (2018) 052005, [arXiv:1802.04146].

[179] ATLAS, G. Aad et al., A search for the $Z \gamma$ decay mode of the Higgs boson in pp collisions at $\sqrt{s}=13$ TeV with the ATLAS detector, Phys. Lett. B 809 (2020) 135754, [arXiv:2005.05382].

[180] CMS, A. M. Sirunyan et al., Search for the decay of a Higgs boson in the $\ell \ell \gamma$ channel in proton-proton collisions at $\sqrt{s}=13 \mathrm{TeV}$, JHEP 11 (2018) 152, [arXiv: 1806.05996].

[181] R. Cahn, M. S. Chanowitz, and N. Fleishon, Higgs Particle Production by Z $\rightarrow$ H Gamma, Phys. Lett. B 82 (1979) 113-116.

[182] L. Bergstrom and G. Hulth, Induced Higgs Couplings to Neutral Bosons in $e^{+} e^{-}$Collisions, Nucl. Phys. B 259 (1985) 137-155. [Erratum: Nucl.Phys.B 276, 744-744 (1986)].

[183] M. Spira, A. Djouadi, and P. Zerwas, QCD corrections to the H Z gamma coupling, Phys. Lett. B 276 (1992) 350-353.

[184] R. Bonciani, V. Del Duca, H. Frellesvig, J. M. Henn, F. Moriello, and V. A. Smirnov, Next-to-leading order $Q C D$ corrections to the decay width $H \rightarrow Z \gamma$, JHEP 08 (2015) 108, [arXiv: 1505.00567].

[185] T. Gehrmann, S. Guns, and D. Kara, The rare decay $H \rightarrow Z \gamma$ in perturbative QCD, JHEP 09 (2015) 038, [arXiv:1505.00561].

[186] L. Hue, A. Arbuzov, T. Hong, T. P. Nguyen, D. Si, and H. Long, General one-loop formulas for decay $h \rightarrow Z \gamma$, Eur. Phys. J. C 78 (2018), no. 11 885, [arXiv: 1712.05234].

[187] B. Bellazzini and F. Riva, New phenomenological and theoretical perspective on anomalous ZZ and $Z \gamma$ processes, Phys. Rev. D 98 (2018), no. 9 095021, [arXiv:1806.09640].

[188] A. Dedes, K. Suxho, and L. Trifyllis, The decay $h \rightarrow Z \gamma$ in the Standard-Model Effective Field Theory, JHEP 06 (2019) 115, [arXiv: 1903.12046].

[189] T. J. Weiler and T.-C. Yuan, Looking for SUSY Virtually Through Loop Induced Higgs Processes, Nucl. Phys. B 318 (1989) 337-374. 
[190] C.-W. Chiang and K. Yagyu, Higgs boson decays to $\gamma \gamma$ and $Z \gamma$ in models with Higgs extensions, Phys. Rev. D 87 (2013), no. 3 033003, [arXiv:1207.1065].

[191] J. Cao, L. Wu, P. Wu, and J. M. Yang, The Z+photon and diphoton decays of the Higgs boson as a joint probe of low energy SUSY models, JHEP 09 (2013) 043, [arXiv: 1301.4641].

[192] N. Maru and N. Okada, $H \rightarrow Z \gamma$ in gauge-Higgs unification, Phys. Rev. D 88 (2013), no. 3 037701, [arXiv:1307.0291].

[193] C.-X. Yue, Q.-Y. Shi, and T. Hua, Vector bileptons and the decays $h \rightarrow \gamma \gamma, Z \gamma$, Nucl. Phys. B 876 (2013) 747-757, [arXiv:1307.5572].

[194] G. Belanger, V. Bizouard, and G. Chalons, Boosting Higgs boson decays into gamma and $a$ in the NMSSM, Phys. Rev. D 89 (2014), no. 9 095023, [arXiv:1402.3522].

[195] D. Fontes, J. Romão, and J. a. P. Silva, $h \rightarrow Z \gamma$ in the complex two Higgs doublet model, JHEP 12 (2014) 043, [arXiv:1408.2534].

[196] A. Hammad, S. Khalil, and S. Moretti, Higgs boson decays into $\gamma \gamma$ and $Z \gamma$ in the MSSM and the B-L supersymmetric SM, Phys. Rev. D 92 (2015), no. 9 095008, [arXiv: 1503.05408].

[197] H. Hung, T. Hong, H. Phuong, H. Mai, and L. Hue, Neutral Higgs decays $H \rightarrow Z \gamma, \gamma \gamma$ in 3-3-1 models, Phys. Rev. D 100 (2019), no. 7075014 , [arXiv:1907.06735].

[198] C.-X. Liu, H.-B. Zhang, J.-L. Yang, S.-M. Zhao, Y.-B. Liu, and T.-F. Feng, Higgs boson decay $h \rightarrow Z \gamma$ and muon magnetic dipole moment in the $\mu \nu S S M$, JHEP 04 (2020) 002, [arXiv:2002.04370].

[199] S.-P. He, Higgs boson to $\gamma Z$ decay as a probe of flavor-changing neutral Yukawa couplings, Phys. Rev. D 102 (2020), no. 7 075035, [arXiv:2004.12155].

[200] P. Bhupal Dev, D. K. Ghosh, N. Okada, and I. Saha, 125 GeV Higgs Boson and the Type-II Seesaw Model, JHEP 03 (2013) 150, [arXiv:1301.3453]. [Erratum: JHEP 05, 049 (2013)].

[201] Q.-H. Cao, L.-X. Xu, B. Yan, and S.-H. Zhu, Signature of pseudo Nambu-Goldstone Higgs boson in its decay, Phys. Lett. B 789 (2019) 233-237, [arXiv: 1810.07661].

[202] N. Bizot and M. Frigerio, Fermionic extensions of the Standard Model in light of the Higgs couplings, JHEP 01 (2016) 036, [arXiv: 1508.01645]. 
[203] J. M. No and M. Spannowsky, A Boost to $h \rightarrow Z \gamma$ : from LHC to Future $e^{+} e^{-}$ Colliders, Phys. Rev. D 95 (2017), no. 7 075027, [arXiv:1612.06626].

[204] Q.-H. Cao, H.-R. Wang, and Y. Zhang, Probing $H Z \gamma$ and $H \gamma \gamma$ anomalous couplings in the process $e^{+} e^{-} \rightarrow H \gamma$, Chin. Phys. C 39 (2015), no. 11 113102, [arXiv: 1505.00654].

[205] J. R. Ellis, M. K. Gaillard, and D. V. Nanopoulos, A Phenomenological Profile of the Higgs Boson, Nucl. Phys. B106 (1976) 292.

[206] M. A. Shifman, A. Vainshtein, M. Voloshin, and V. I. Zakharov, Low-energy theorems for higgs boson couplings to photons, Sov. J. Nucl. Phys. 30 (1979), no. ITEP-42-1979 1368-1378.

[207] D. Ross and M. Veltman, Neutral Currents in Neutrino Experiments, Nucl. Phys. B 95 (1975) 135-147.

[208] M. Veltman, Limit on Mass Differences in the Weinberg Model, Nucl. Phys. B 123 (1977) 89-99.

[209] S. Braibant, SUSY searches at LEP, in 38th Rencontres de Moriond on QCD and High-Energy Hadronic Interactions, 5, 2003. hep-ex/0305058.

[210] ALEPH, A. Heister et al., Search for scalar quarks in $e^{+} e^{-}$collisions at $\sqrt{s}$ up to 209-GeV, Phys. Lett. B 537 (2002) 5-20, [hep-ex/0204036].

[211] DELPHI, J. Abdallah et al., Searches for supersymmetric particles in $e+e-$ collisions up to 208-GeV and interpretation of the results within the MSSM, Eur. Phys. J. C 31 (2003) 421-479, [hep-ex/0311019].

[212] L3, P. Achard et al., Search for scalar leptons and scalar quarks at LEP, Phys. Lett. B 580 (2004) 37-49, [hep-ex/0310007].

[213] OPAL, G. Abbiendi et al., Search for scalar top and scalar bottom quarks at LEP, Phys. Lett. B 545 (2002) 272-284, [hep-ex/0209026]. [Erratum: Phys.Lett.B 548, 258-258 (2002)].

[214] S. Bolognesi, Y. Gao, A. V. Gritsan, K. Melnikov, M. Schulze, N. V. Tran, and A. Whitbeck, On the spin and parity of a single-produced resonance at the LHC, Phys. Rev. D 86 (2012) 095031, [arXiv:1208.4018].

[215] D. Stolarski and R. Vega-Morales, Directly Measuring the Tensor Structure of the Scalar Coupling to Gauge Bosons, Phys. Rev. D 86 (2012) 117504, [arXiv:1208.4840].

[216] Y. Chen and R. Vega-Morales, Extracting Effective Higgs Couplings in the Golden Channel, JHEP 04 (2014) 057, [arXiv: 1310.2893]. 
[217] Y. Chen, R. Harnik, and R. Vega-Morales, Probing the Higgs Couplings to Photons in h $\rightarrow$ \&l at the LHC, Phys. Rev. Lett. 113 (2014), no. 19 191801, [arXiv:1404.1336].

[218] A. Falkowski and R. Vega-Morales, Exotic Higgs decays in the golden channel, JHEP 12 (2014) 037, [arXiv: 1405.1095].

[219] Y. Chen, D. Stolarski, and R. Vega-Morales, Golden probe of the top Yukuwa coupling, Phys. Rev. D92 (2015), no. 5 053003, [arXiv:1505.01168].

[220] Y. Chen, J. Lykken, M. Spiropulu, D. Stolarski, and R. Vega-Morales, Golden Probe of Electroweak Symmetry Breaking, Phys. Rev. Lett. 117 (2016), no. 24 241801, [arXiv: 1608.02159].

[221] S. Boselli, C. M. Carloni Calame, G. Montagna, O. Nicrosini, F. Piccinini, and A. Shivaji, Higgs decay into four charged leptons in the presence of dimension-six operators, JHEP 01 (2018) 096, [arXiv:1703.06667].

[222] I. Anderson et al., Constraining Anomalous HVV Interactions at Proton and Lepton Colliders, Phys. Rev. D 89 (2014), no. 3 035007, [arXiv:1309.4819].

[223] J. S. Gainer, J. Lykken, K. T. Matchev, S. Mrenna, and M. Park, Beyond Geolocating: Constraining Higher Dimensional Operators in $H \rightarrow 4 \ell$ with Off-Shell Production and More, Phys. Rev. D91 (2015), no. 3 035011, [arXiv: 1403.4951].

[224] D. Liu, I. Low, and R. Vega-Morales, A Golden Probe of Nonlinear Higgs Dynamics, Eur. Phys. J. C 80 (2020), no. 9 829, [arXiv:1904.00026].

[225] CMS, A. M. Sirunyan et al., Constraints on anomalous Higgs boson couplings using production and decay information in the four-lepton final state, Phys. Lett. B 775 (2017) 1-24, [arXiv: 1707.00541].

[226] Y. Chen, R. Harnik, and R. Vega-Morales, New opportunities in $h \rightarrow 4 l$, JHEP 09 (2015) 185, [arXiv: 1503.05855].

[227] J. Fan and M. Reece, A New Look at Higgs Constraints on Stops, JHEP 06 (2014) 031, [arXiv:1401.7671].

[228] G. Burdman, Z. Chacko, H.-S. Goh, and R. Harnik, Folded supersymmetry and the LEP paradox, JHEP 02 (2007) 009, [hep-ph/0609152].

[229] LHC Higgs Cross Section Working Group, J. R. Andersen et al., Handbook of LHC Higgs Cross Sections: 3. Higgs Properties, arXiv:1307.1347. 
[230] M. Cepeda et al., Report from Working Group 2: Higgs Physics at the HL-LHC and HE-LHC, CERN Yellow Rep. Monogr. 7 (2019) 221-584, [arXiv: 1902.00134].

[231] Y. Chen, E. Di Marco, J. Lykken, M. Spiropulu, R. Vega-Morales, and S. Xie, $8 D$ Likelihood Effective Higgs Couplings Extraction Framework in $h \rightarrow 4 \ell$, JHEP 01 (2015) 125, [arXiv:1401.2077].

[232] A. Bredenstein, A. Denner, S. Dittmaier, and M. M. Weber, Precise predictions for the Higgs-boson decay $\mathrm{H} \longrightarrow \mathrm{WW} / \mathrm{ZZ} \rightarrow 4$ leptons, Phys. Rev. D 74 (2006) 013004, [hep-ph/0604011].

[233] A. Bredenstein, A. Denner, S. Dittmaier, and M. M. Weber, Radiative corrections to the semileptonic and hadronic Higgs-boson decays $H \rightarrow W W$ / ZZ > 4 fermions, JHEP 02 (2007) 080, [hep-ph/0611234].

[234] I. Low, J. Lykken, and G. Shaughnessy, Have We Observed the Higgs (Imposter)?, Phys. Rev. D86 (2012) 093012, [arXiv: 1207.1093].

[235] J. Brod, U. Haisch, and J. Zupan, Constraints on CP-violating Higgs couplings to the third generation, JHEP 11 (2013) 180, [arXiv:1310.1385].

[236] M. Farina, Y. Grossman, and D. J. Robinson, Probing $C P$ violation in $h \rightarrow Z \gamma$ with background interference, Phys. Rev. D 92 (2015), no. 7 073007, [arXiv:1503.06470].

[237] X. Chen, G. Li, and X. Wan, Probe CP violation in $H \rightarrow \gamma Z$ through forward-backward asymmetry, Phys. Rev. D 96 (2017), no. 5 055023, [arXiv: 1705.01254].

[238] Y. Chen, A. Falkowski, I. Low, and R. Vega-Morales, New Observables for CP Violation in Higgs Decays, Phys. Rev. D 90 (2014), no. 11 113006, [arXiv: 1405.6723].

[239] M. Gonzalez-Alonso, A. Greljo, G. Isidori, and D. Marzocca, Pseudo-observables in Higgs decays, Eur. Phys. J. C75 (2015) 128, [arXiv: 1412.6038].

[240] J. Alwall, R. Frederix, S. Frixione, V. Hirschi, F. Maltoni, O. Mattelaer, H. S. Shao, T. Stelzer, P. Torrielli, and M. Zaro, The automated computation of tree-level and next-to-leading order differential cross sections, and their matching to parton shower simulations, JHEP 07 (2014) 079, [arXiv: 1405.0301].

[241] LHC Higgs Cross Section Working Group, S. Dittmaier et al., Handbook of LHC Higgs Cross Sections: 1. Inclusive Observables, arXiv:1101.0593. 
[242] CMS, A. M. Sirunyan et al., Measurements of production cross sections of the Higgs boson in the four-lepton final state in proton-proton collisions at $\sqrt{s}=$ 13 TeV, arXiv:2103.04956.

[243] Y. Gao, A. V. Gritsan, Z. Guo, K. Melnikov, M. Schulze, and N. V. Tran, Spin Determination of Single-Produced Resonances at Hadron Colliders, Phys. Rev. D 81 (2010) 075022, [arXiv:1001.3396].

[244] K. Hally, H. E. Logan, and T. Pilkington, Constraints on large scalar multiplets from perturbative unitarity, Phys. Rev. D 85 (2012) 095017, [arXiv: 1202.5073].

[245] S. Weinberg, Baryon and Lepton Nonconserving Processes, Phys. Rev. Lett. 43 (1979) 1566-1570.

[246] W. Buchmuller and D. Wyler, Effective Lagrangian Analysis of New Interactions and Flavor Conservation, Nucl. Phys. B 268 (1986) 621-653.

[247] C. N. Leung, S. Love, and S. Rao, Low-Energy Manifestations of a New Interaction Scale: Operator Analysis, Z. Phys. C 31 (1986) 433.

[248] B. Grzadkowski, M. Iskrzynski, M. Misiak, and J. Rosiek, Dimension-Six Terms in the Standard Model Lagrangian, JHEP 10 (2010) 085, [arXiv: 1008.4884].

[249] G. Branco, P. Ferreira, L. Lavoura, M. Rebelo, M. Sher, and J. P. Silva, Theory and phenomenology of two-Higgs-doublet models, Phys. Rept. 516 (2012) 1-102, [arXiv:1106.0034].

[250] ATLAS, G. Aad et al., Measurements of the Higgs boson inclusive and differential fiducial cross sections in the 4 l decay channel at $\sqrt{s}=13 \mathrm{TeV}$, arXiv:2004.03969.

[251] B. Schmidt, The high-luminosity upgrade of the LHC: Physics and technology challenges for the accelerator and the experiments, Journal of Physics: Conference Series 706 (apr, 2016) 022002.

[252] F. Dulat, A. Lazopoulos, and B. Mistlberger, iHixs 2 - Inclusive Higgs cross sections, Comput. Phys. Commun. 233 (2018) 243-260, [arXiv:1802.00827].

[253] Proc. QCD, EW, and tools at $100 \mathrm{TeV}$, Introduction, (Geneva, Switzerland), ATLAS, 2015.

[254] LHC Higgs Cross Section Working Group, D. de Florian et al., Handbook of LHC Higgs Cross Sections: 4. Deciphering the Nature of the Higgs Sector, arXiv:1610.07922. 
[255] T. Falk, K. A. Olive, and M. Srednicki, Phases in the MSSM, electric dipole moments and cosmological dark matter, Phys. Lett. B 354 (1995) 99-106, [hep-ph/9502401].

[256] R. Contino et al., Physics at a $100 \mathrm{TeV}$ pp collider: Higgs and EW symmetry breaking studies, CERN Yellow Rep. (2017), no. 3 255-440, [arXiv: 1606.09408].

[257] A. Djouadi, The Anatomy of electro-weak symmetry breaking. II. The Higgs bosons in the minimal supersymmetric model, Phys. Rept. 459 (2008) 1-241, [hep-ph/0503173].

[258] M. Carena, I. Low, and C. E. M. Wagner, Implications of a Modified Higgs to Diphoton Decay Width, JHEP 08 (2012) 060, [arXiv: 1206.1082]. 